\title{
A Multi-Scale Approach to Exploiting Measured and Modelled Building Performance Data to Improve Campus Operations
}

\author{
by \\ Aly Assem Abdelalim, M.A.Sc. \\ A thesis submitted to the \\ Faculty of Graduate and Postdoctoral Affairs \\ in partial fulfillment of the requirements for the degree of
}

\section{Doctor of Philosophy Engineering}

\author{
Ottawa-Carleton Institute for Environmental Engineering \\ Department of Civil and Environmental Engineering Carleton University \\ Ottawa, Ontario \\ April, 2017
}

(C) Copyright

Aly Assem Abdelalim, 2017 
The undersigned hereby recommends to the Faculty of Graduate and Postdoctoral Affairs acceptance of the thesis

\title{
A Multi-Scale Approach to Exploiting Measured and Modelled Building Performance Data to Improve Campus Operations
}

\author{
submitted by Aly Assem Abdelalim, M.A.Sc. \\ in partial fulfillment of the requirements for the degree of \\ Doctor of Philosophy Engineering

\begin{tabular}{c} 
Professor David Layzell \\
External Examiner \\
Professor William O'Brien \\
\hline Thesis Supervisor \\
Professor Stephen Fai \\
Internal Examiner \\
Professor Ian Beausoleil-Morrison \\
Internal Examiner \\
Professor Scott Bucking \\
Internal Examiner
\end{tabular}

Professor Paul Van Geel, Chair, Department of Civil and Environmental Engineering

Ottawa-Carleton Institute for Environmental Engineering

Department of Civil and Environmental Engineering

Carleton University

April, 2017 


\begin{abstract}
Campuses, communities, and other building clusters are major users of energy and water and thus can have a significant environmental impact. Frequently, buildings' resource consumption is tracked at various levels of spatial and temporal resolution through meters, in order to monitor and reduce resource consumption. However, the metering and data logging systems are often inconvenient and difficult to access due to use of multiple systems and technologies of varying vintages. Moreover, modern commercial buildings have complex mechanical systems and heat transfer paths, and these are typically difficult to visualize. Furthermore, current data availability and visualization tools do not lend themselves to identification of inefficiencies and possible solutions.
\end{abstract}

This work is divided into two main parts. The aim of the first part is to provide a useful workflow and set of methods to enhance campus operations. Within the scope of this work, by using a combination of measured data and models, a comprehensive energy use assessment at different scales can be formed. This information can yield greater insights about opportunities for operational improvements and retrofits that would not be available through measurements alone. This work involves the application and testing on Carleton University campus and its Canal Building to validate the theory using real-world case study. It also allows the usability to be tested on real stakeholders (building operators, campus planners, architects and accountants).

The second part deals with practical elements of application and dissemination. In this part, a workflow is developed to automate the process of creating Sankey diagrams from energy simulation outputs. Moreover, this part investigates the feasibility of utilizing the 
visualization technique (Sankey diagrams) developed in the first part to evaluate various design variants and to enhance the decision-making process.

The main contributions of this research include methodologies to: 1) convert sparse sensor and sub-meter data into estimated energy flows, 2) combine measured and modeled data to provide a detailed record of buildings and campus resource consumption at a wide range of scales, 3) convert building information models (BIM) into energy models, 4) combine hybrid evidence-based, analytical optimization, and inverse calibration methods, 5) estimate the impact of unmeasured energy flows, 6) estimate upstream environmental impacts of buildings and campuses, and 7) visualize measured and modelled data using Sankey diagrams at various scales: from building system to campus level. 


\section{Acknowledgments}

I would like to express my deepest appreciation to all those who provided me the possibility to complete this work. A special gratitude I give to Dr. Liam O'Brien, whose contribution in stimulating suggestions and encouragement, helped me to coordinate my project especially in writing this thesis. I would like to acknowledge my co-workers at Carleton Building Research Groups: Dr. Burak Gunay, Shawn Shi, Jayson Bursill, Dr. Sara Gilani, Tareq Abuimara, Isis Bennet, Chris Baldwin, Zach Burgoyne, Justin Berquist, Austin Selvig, Andrew Hicks, Mihai Mateescu, and Dr. Scott Bucking. I would like to acknowledge Prof. Stephen Fai for his generous effort and support in accomplishing this work.

I would also like to acknowledge with much appreciation the generous support of Autodesk (Azam Khan, Ramtin Attar, and Alex Tessier), Natural Sciences and Engineering Research Council (NSERC), and Natural Resources Canada (Phylroy Lopez and Meli Stylianou). Furthermore, this research would not be possible without the tireless ongoing support of Carleton University's Facilities Management and Planning (Darryl Boyce, Lisa

Paterick, and Scott Macdonald). I would also like to express my deepest appreciation to my brother Abdelrahman for his support in developing the code that automates the creation of Sankey diagrams.

Finally, thank you to my family for all their support and encouragement through not just this process but everything leading up until this point. A special thanks to my beloved wife for her support and taking care of my son to be able to complete my $\mathrm{PhD}$. She also sacrificed with her time to take care of my family during my absence. 


\section{Table of Contents}

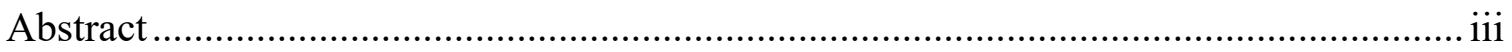

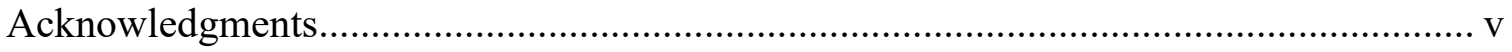

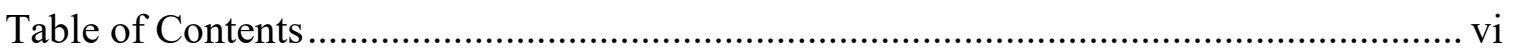

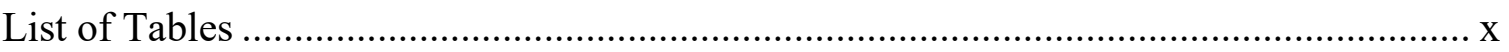

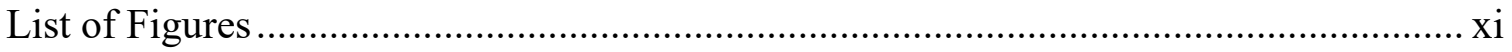

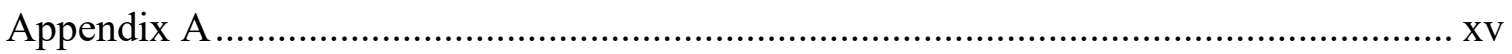

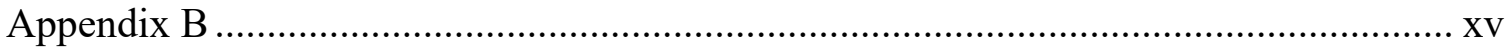

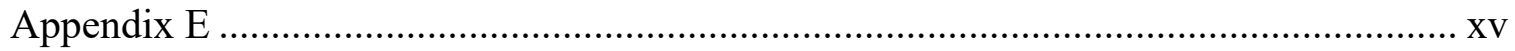

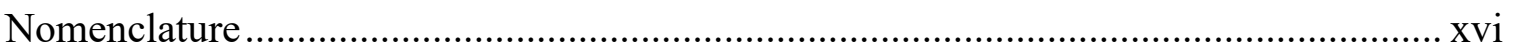

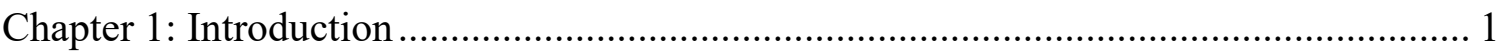

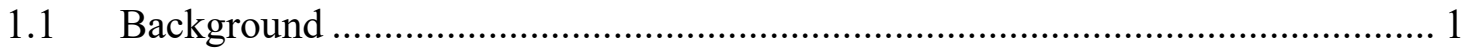

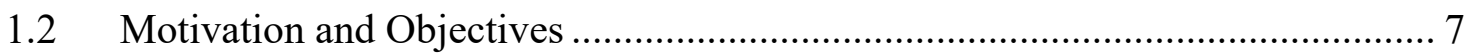

1.3 Research Methodology............................................................................ 9

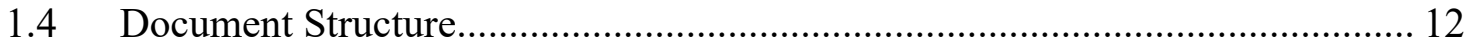

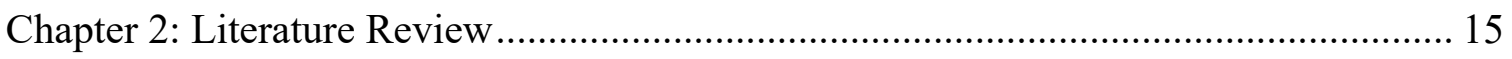

2.1 Campus Metabolism............................................................................ 15

2.2 Measured Data Synthesis ............................................................................ 16

2.3 Interoperability between BIM and Energy Analysis Tools................................ 17

2.3.1 BIM Protocols ................................................................................ 18

2.3.2 Interoperability between BIM and EnergyPlus......................................... 19

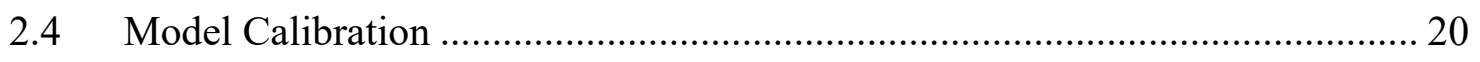

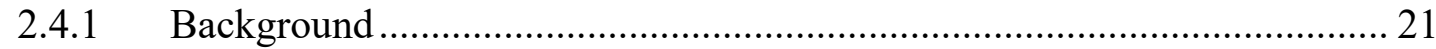

2.4.2 Existing Building Energy Simulation Models ........................................... 22

2.4.3 Previous Work and Existing Calibration Methodologies ............................ 24

2.4.4 Selection of Calibration Parameters.............................................................. 30

2.4.5 Accuracy of Calibrated BPS Models ...................................................... 32

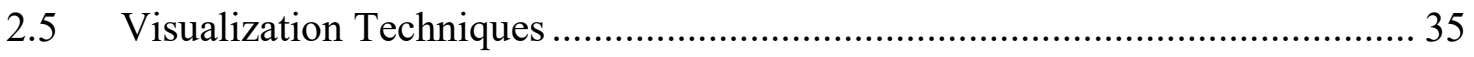

2.5.1 Current Building Energy Visualization Tools ............................................ 35

2.5.2 Current Visualization Techniques used for Parametric Design of Buildings 


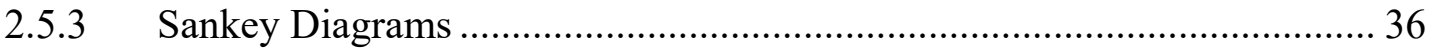

2.5.4 Methods for Evaluating Visualization Techniques..................................... 37

Part I: Developing Methods to Enhance Campus Operations.................................... 41

Chapter 3: Visualization of Energy, Water Consumption and GHG Emissions ............... 42

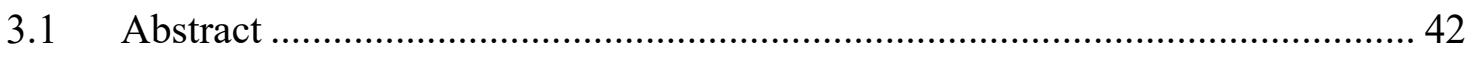

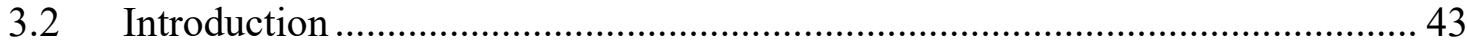

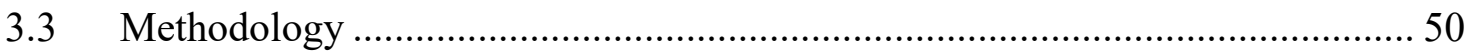

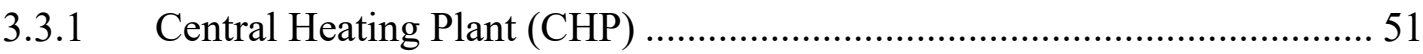

3.3.2 Accounting for Building Size and Energy Use Intensity ............................ 53

3.3.3 Primary to Secondary Conversion Factors …………………………….... 54

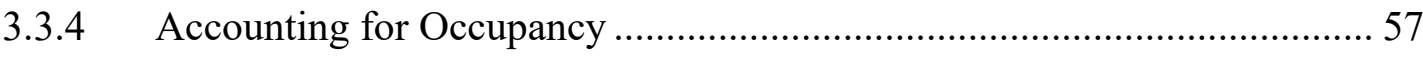

3.3.5 Accounting for Building Age and Usage.................................................. 58

3.3.6 Calculations for Equivalent Carbon Dioxide Emissions (CO2e) ……........ 58

3.4 Application: Carleton University Campus ……………………...................... 59

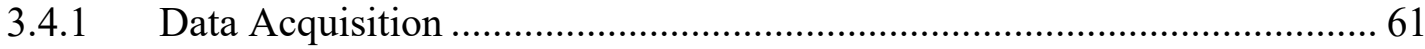

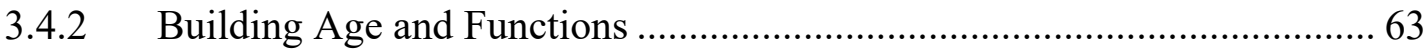

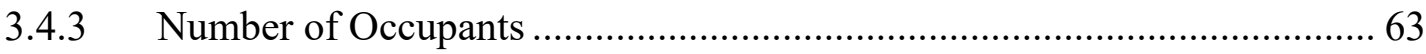

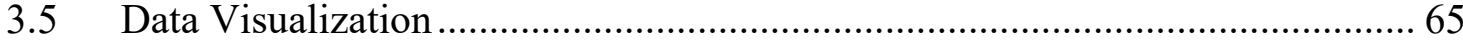

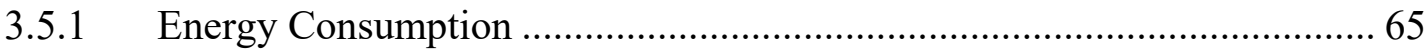

3.5.2 Mass flow (District Water, Steam, and Natural Gas) .................................. 66

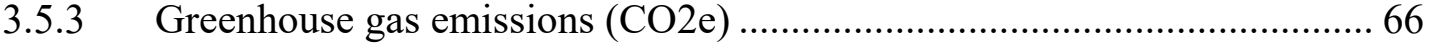

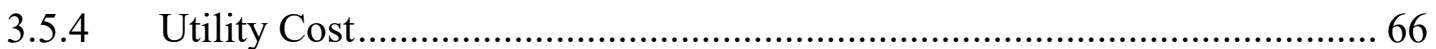

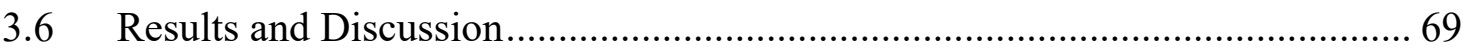

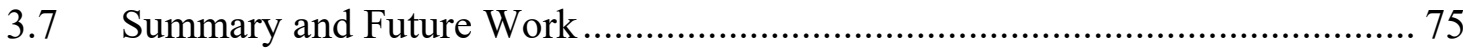

Chapter 4: Data Visualization and Analysis of Energy Flow on a Multi-Zone Building

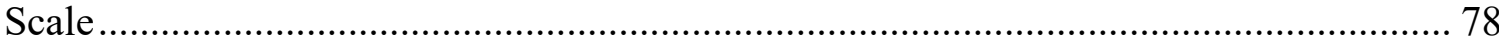

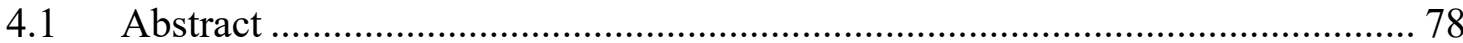

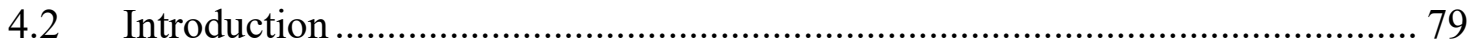

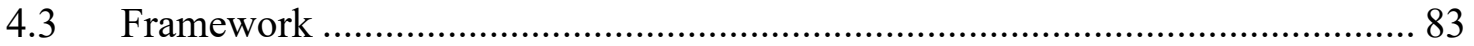

4.3.1 Convert BIM based Architectural Model to Building Performance Model 85 


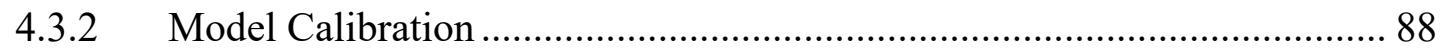

4.3.3 Modeling Approach in EnergyPlus ( $\mathrm{E}+$ ) .................................................. 92

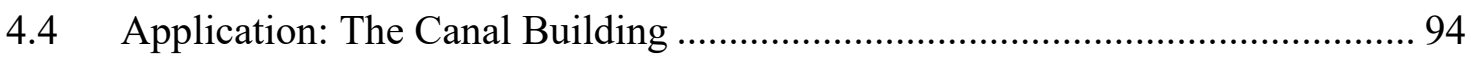

4.4.1 Building Performance Model Development ............................................... 95

4.4.2 Model calibration Results ........................................................................ 97

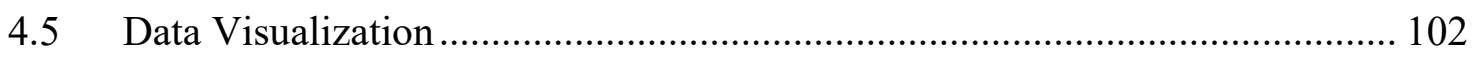

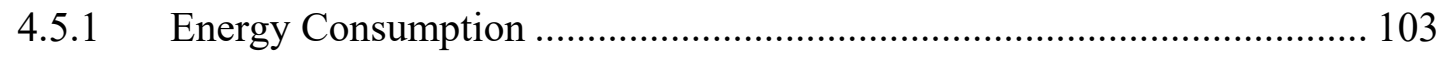

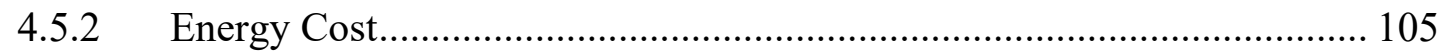

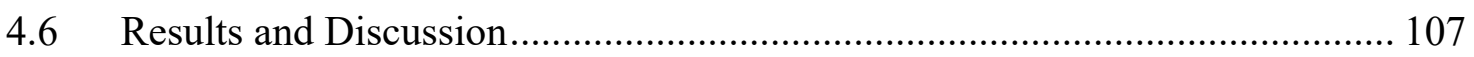

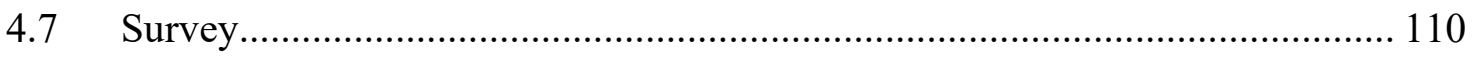

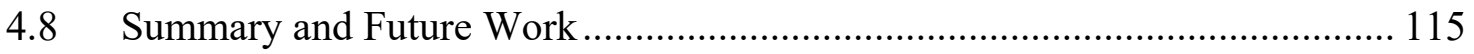

Chapter 5: Development of Sankey Diagrams to Visualize Real HVAC Performance . 117

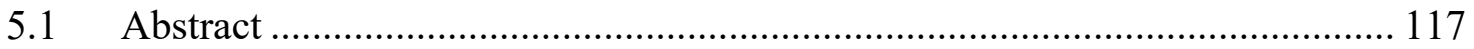

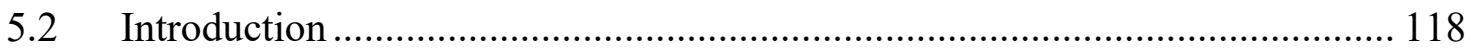

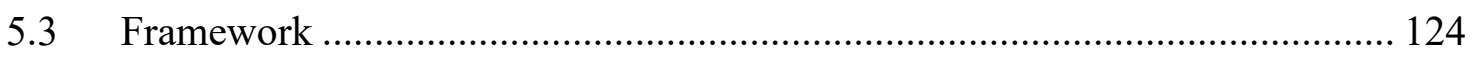

5.3.1 Central Heating Plant (CHP) ………………...................................... 126

5.3.2 Cooling Towers.............................................................................. 127

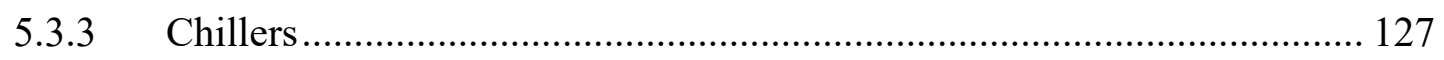

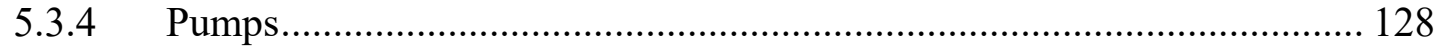

5.3.5 Air Handling Unit (AHU) .................................................................... 129

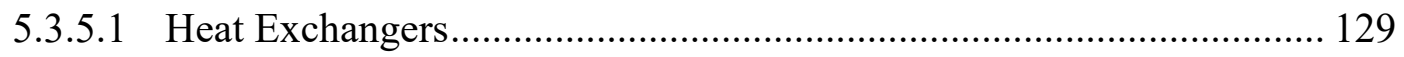

5.3.5.2 Heat Recovery Wheel (HRW) ......................................................... 130

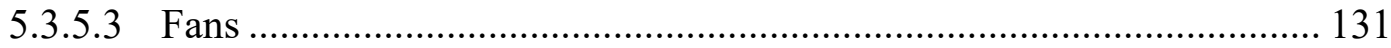

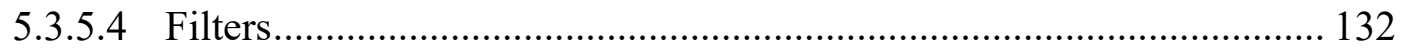

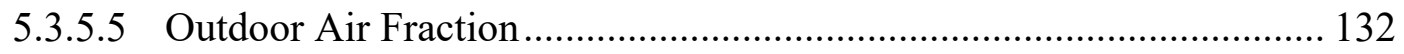

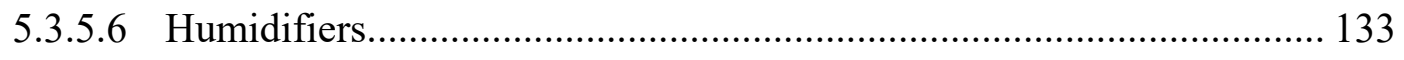

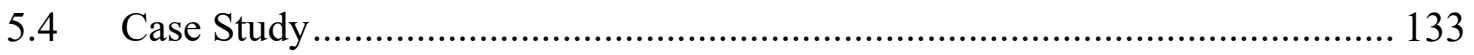

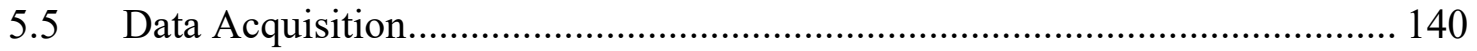

5.6 Data Visualization............................................................................. 145

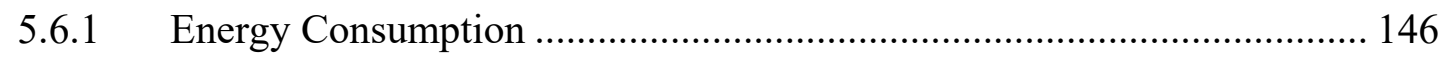

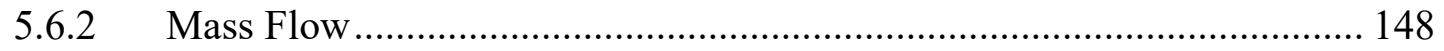




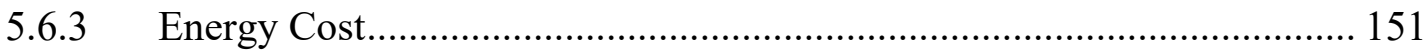

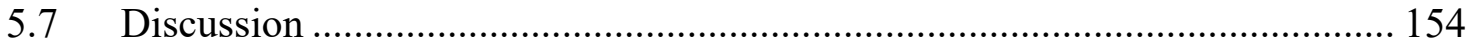

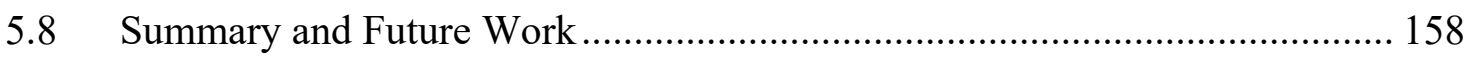

Part II: Implementation of Sankey Diagrams as A Decision-Making Tool ............ 160

Chapter 6: Visualization of Building Performance using Sankey Diagrams to Enhance the

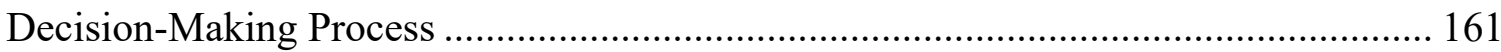

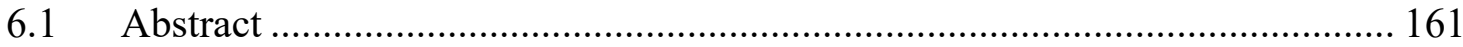

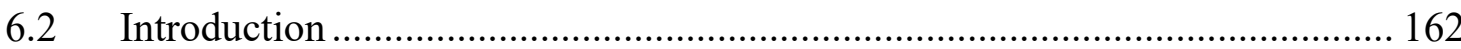

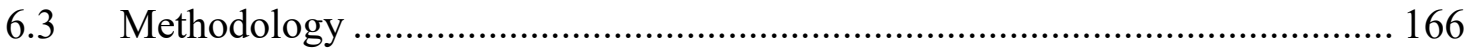

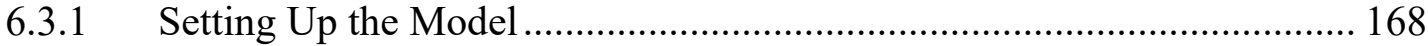

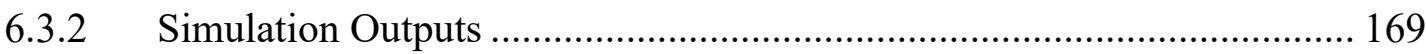

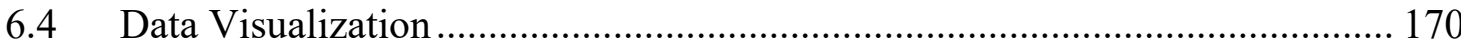

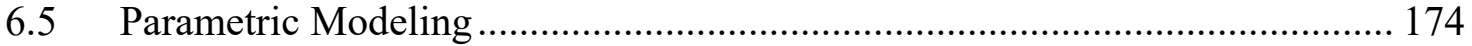

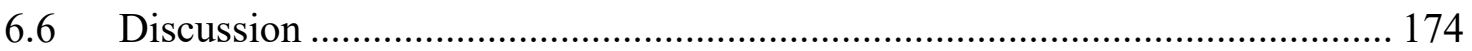

6.7 Summary and Future Work ....................................................................... 184

Chapter 7: Automating the Creation of Sankey Diagrams ........................................... 185

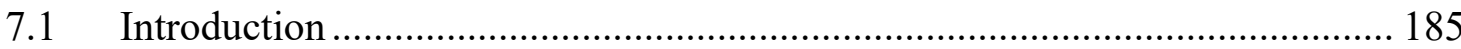

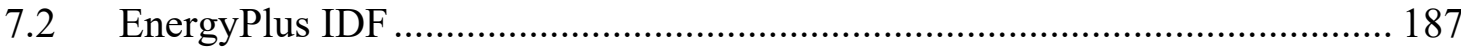

7.3 Simulation Output Variables and Meters …………………………………..... 190

7.4 Front-end Implementation............................................................................. 192

7.4.1 Hypertext Markup Language (HTML) .................................................... 192

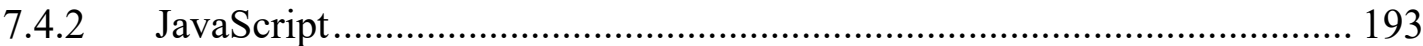

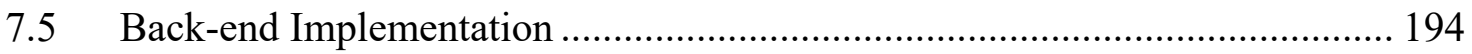

7.5.1 Hypertext Preprocessor (PHP) ……………………………………....... 194

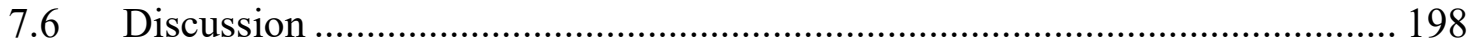

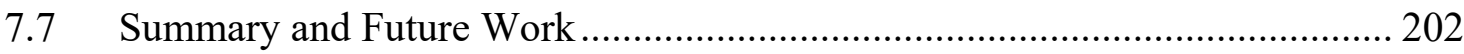

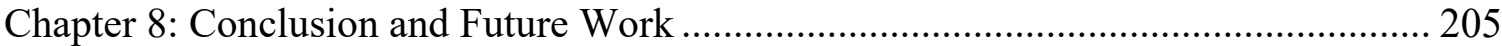

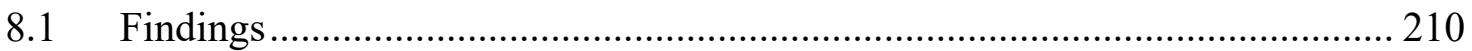

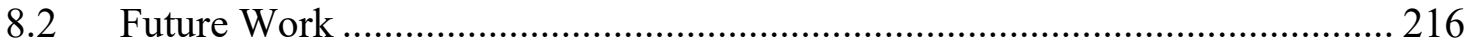

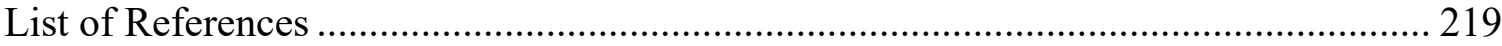

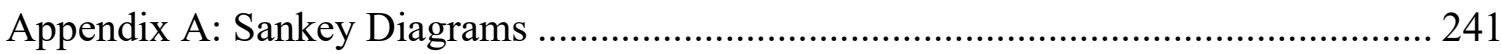


Appendix B: Normalized data per unit area and occupant ............................................. 244

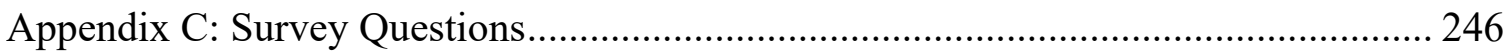

Appendix D: Hypertext Markup Language (HTML) Code …………………………... 253

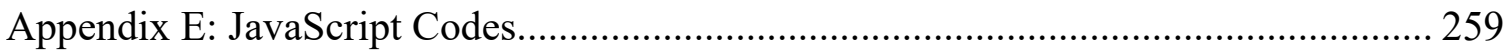

Appendix F: Hypertext Preprocessor (PHP) Code …………………………………... 284

Appendix G: Copyright License Agreements............................................................. 322

Appendix H: Copyright License Agreements............................................................ 323

\section{List of Tables}

Table 2.1: Source of uncertainty in building energy models [72] .................................. 31

Table 2.2: Calibration tolerances by different standards ............................................... 34

Table 2.3: End-use specific tolerances (adapted from Kaplan et al. [47])........................ 34

Table 2.4: Comparison between different visualization techniques used in BPS tools.... 39

Table 3.1: Secondary to primary conversion factors ………………................................ 56

Table 3.2: CO2e Emission Factors ……………………............................................... 59

Table 3.3: Building name corresponding to building number ......................................... 62

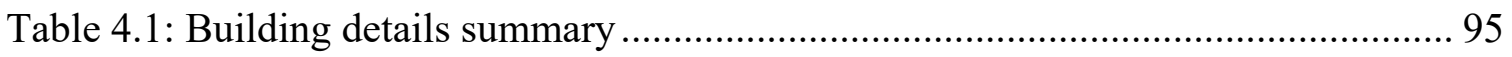

Table 4.2: Parameters used for optimization .............................................................. 102

Table 5.1: Air handling unit and plant loops details summary ...................................... 136

Table 5.2: Sensors and sub-meters' details summary .................................................. 141

Table 6.1: Base case model details summary .......................................................... 168

Table 6.2: Hourly simulation output variables used in the study .................................... 169

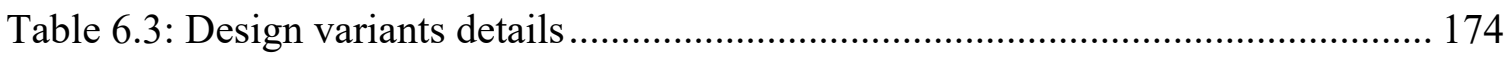

Table 7.1: Base case model details summary ............................................................. 189

Table 7.2: Hourly simulation output variables and meters used in the study ................ 190 


\section{List of Figures}

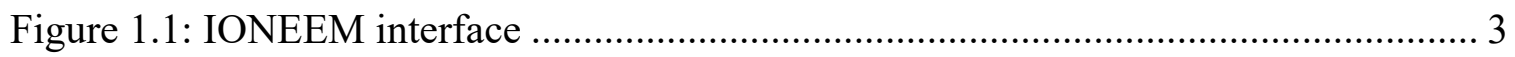

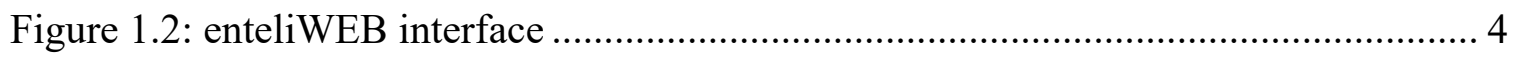

Figure 1.3: Sankey diagrams showing energy supply and demand on campus level........ 6

Figure 1.4: Flowchart showing the overall framework to analyze and visualize energy flows, GHGs, and the corresponding energy cost on different scales (campus, building,

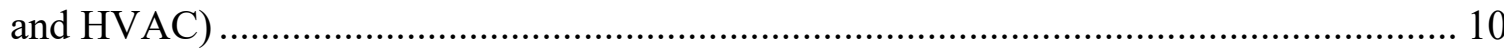

Figure 2.1: The proposed methodology to estimate and visualize energy flows obtained from sensors and sub-meters on campus, building-level, and HVAC system.................. 15

Figure 2.2: Inverse modeling (Adapted from Krarti [38]) ............................................. 23

Figure 2.3: Forward modeling (Adapted from Krarti [38]) ……………....................... 23

Figure 2.4: visualization of national energy supply, demand, and exports using Sankey

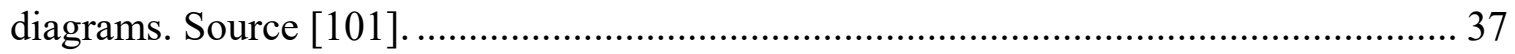

Figure 3.1: Flowchart showing the main scope of the study ........................................... 51

Figure 3.2: Energy and water consumption by CHP and different forms of heat losses.. 52

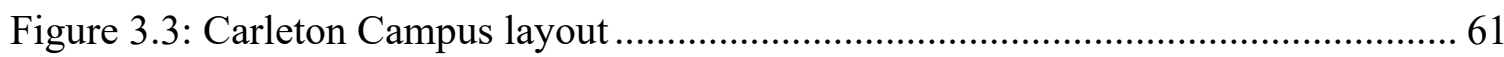

Figure 3.4: Number of occupants and area for each building........................................ 65

Figure 3.5: Sankey diagram showing annual energy consumption ................................ 67

Figure 3.6: Normalized annual energy consumption and $\mathrm{CO} 2 \mathrm{e}$ emissions per unit area. 68

Figure 3.7: Normalized annual energy consumption and $\mathrm{CO} 2 \mathrm{e}$ emissions per person .... 68

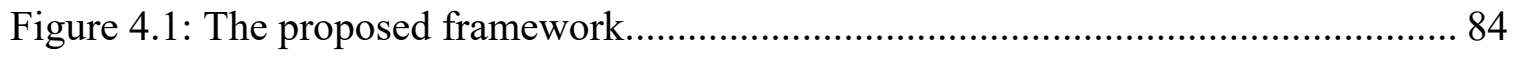

Figure 4.2: Flowchart showing the main scope of the study on the building level .......... 85

Figure 4.3: Building Model Development Process ........................................................ 86

Figure 4.4: The building energy model calibration method to achieve (MBE) of $\pm 10 \%$ and CV (RMSE) of $\pm 30 \%$ for hourly based calibration, as well as achieving (MBE) of \pm $5 \%$ and $\mathrm{CV}$ (RMSE) of $\pm 15 \%$ for monthly based calibration 89 
Figure 4.5: The inverse calibration procedure

Figure 4.6: The conversion process from BIM (left) to analytical model (middle) to BPS tools (DesignBuilder) (right) 96

Figure 4.7: The Canal Building fourth floor showing different zone activities and merged zones (same activity and boundary conditions) within dotted lines 97

Figure 4.8: Comparison between monthly measured and simulated data for heating loads (left) and cooling loads (right) 100

Figure 4.9: Comparison between hourly measured and simulated data for heating rate 100

Figure 4.10: Comparison between hourly measured and simulated data for cooling rate 101

Figure 4.11: Comparison between hourly measured and simulated data during two weeks in winter 101

Figure 4.12: Comparison between hourly measured and simulated data during two weeks in summer. 101

Figure 4.13: Sankey diagram showing building-level energy flow for the winter season, energy flows (for sensed and metered components) colored in red color indicates that the actual energy consumption is higher than the baseline..... 104

Figure 4.14: Sankey diagram showing building-level energy flow for summer season, energy flows (for sensed and metered components) colored in red color or yellow indicates that the actual energy consumption is higher than or equal to the baseline, respectively

Figure 4.15: Sankey diagram showing energy cost during winter season, energy costs (for sensed and metered components) colored in red color indicates that the actual energy consumption is higher than the baseline

Figure 4.16: Sankey diagram showing energy cost during summer season, energy costs (for sensed and metered components) colored in red color or yellow indicates that the actual energy consumption is higher than or equal to the baseline, respectively 106

Figure 4.17: Survey results on the effectiveness of using Sankey diagrams to visualize building-level energy flows

Figure 4.18: Survey results on determining the path responsible for the most cooling (left) and heating (right) energy.... 
Figure 4.19: Survey results on determining the ratio of passive to active energy cost during heating (left) and cooling (right) energy

Figure 4.20: Survey results on determining the building component that would be most effective at reducing combined cost of heating and cooling.....

Figure 4.21: Survey results on evaluating the usefulness of Sankey diagrams to facilitate decision-making

Figure 4.22: Survey results showing how likely the users would use Sankey diagrams to visualize energy flow and cost on the building-level.

Figure 5.1: Energy dashboard for AHU implemented at Carleton University

Figure 5.2: Supply fan hourly energy consumption.

Figure 5.3: Flowchart showing the process to convert historical data to estimated energy and mass flows and the corresponding cost and visualize using Sankey diagrams

Figure 5.4: Typical AHU and plant loops of a commercial building showing actually measured sensors in the selected case study (highlighted in red color) and control volumes (within dotted lines)

Figure 5.6: Actual weather data of Ottawa, Canada in 2015

Figure 5.7: Sankey diagram showing AHU and plant loops energy flow during winter season, energy flows (for sensed and metered components) colored in red color indicates that the actual energy consumption is higher than the baseline.

Figure 5.8: Sankey diagram showing AHU and plant loops energy flow during summer season, energy flows (for sensed and metered components) colored in red color or yellow indicates that the actual energy consumption is higher than or equal to the baseline, respectively

Figure 5.9: Sankey diagram showing AHU and plant loops mass flow during winter season.

Figure 5.10: Sankey diagram showing AHU and plant loops mass flow during summer season.

Figure 5.11: Sankey diagram showing AHU and plant loops energy cost during winter season, energy costs (for sensed and metered components) colored in red color indicates 
that the actual energy consumption is higher than the baseline.....

Figure 5.12: Sankey diagram showing AHU and plant loops energy cost during summer season, energy costs (for sensed and metered components) colored in red color or yellow indicates that the actual energy consumption is higher than or equal to the baseline, respectively 154

Figure 6.1: Flowchart showing the process to visualize energy flows on building-level and HVAC system using Sankey diagrams and evaluate different design variants 167

Figure 6.2: Base case model for the large office building. 169

Figure 6.3: Sankey diagrams showing energy flows on the building-level for the winter season (top) and summer season (bottom) for the base case (BC) 172

Figure 6.4: Sankey diagrams showing energy flows on the HVAC system for the winter season (top) and summer season (bottom) for the base case (BC) 173

Figure 6.5: Sankey diagrams showing energy flows (grey for BC and red for I1) on building-level for the winter season (top) and summer season (bottom).

Figure 6.6: Sankey diagrams showing energy flows (grey for BC and red for I1) on HVAC for the winter season (top) and summer season (bottom). 178

Figure 6.7: Sankey diagrams showing energy flows (grey for BC and blue for I2) on building-level for the winter season (top) and summer season (bottom).

Figure 6.8: Sankey diagrams showing energy flows (grey for BC and blue for I2) on HVAC for the winter season (top) and summer season (bottom) 180

Figure 6.9: Sankey diagrams showing energy flows (grey for BC and green for I3) on building-level for the winter season (top) and summer season (bottom).

Figure 6.10: Sankey diagrams showing energy flows (grey for BC and green for I3) on HVAC for the winter season (top) and summer season (bottom) 182

Figure 7.1: flowchart showing the framework to automate the process of creating Sankey diagrams from EnergyPlus IDF and simulation output (ESO) files 186

Figure 7.2: large (top), medium (middle), and small (bottom) office building models.. 188

Figure 7.3: "HOME" tab showing different user-graphical interface features. 199

Figure 7.4: uploading of ESO file for each diagram 199 
Figure 7.5: "Sankey Diagrams" tab showing different user-graphical interface features

Figure 7.6: multi-layer Sankey diagrams for large office (including base case and case A)

Figure 8.1: The Canal building energy model detailed (left) and simplified (right) ...... 212

Figure 8.2: Comparison between monthly measured data and calibration results of detailed and simplified model data for heating loads (left) and cooling loads (right).... 213

\section{Appendix A}

A. 1: Sankey diagram showing annual mass flow and its distribution by different sources

A. 2: Sankey diagram showing annual $\mathrm{CO} 2 \mathrm{e}$ emissions 242

A. 3: Sankey diagram showing annual utility cost

\section{Appendix B}

B. 1: Normalized district water, natural gas, and steam annual consumption per unit area

B. 2: Normalized district water, natural gas, and steam annual consumption per person

B. 3: Normalized annual utility cost per unit area ………….................................... 245

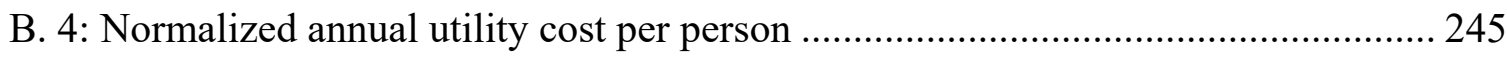

\section{Appendix E}

Appendix E1: JavaScript code providing functions and controls for the "HOME" tab . 259 Appendix E2: D3's Sankey layout JavaScript code ........................................................ 263

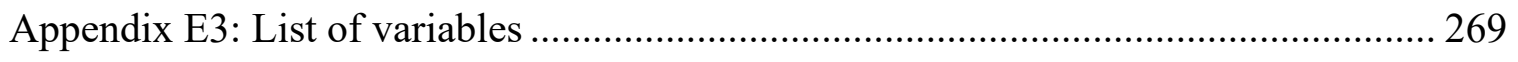

Appendix E4: JavaScript code to generate any number (n) of diagrams........................ 270

Appendix E5: JavaScript code to select spatial resolution .............................................. 275

Appendix E6: JavaScript code to select temporal resolution........................................... 276 
Appendix E7: JavaScript code to animate the results ............................................ 279

Appendix E8: JavaScript code to select color for each diagram .................................. 281

Appendix E9: JavaScript code to Show/hide diagrams ............................................ 282

Appendix E10: JavaScript code to initialize HTML elements in the web page ............. 283 


\section{Nomenclature}

\begin{tabular}{ll}
\hline Abbreviations & \\
\hline ACH 50 pa & Air-Change-Per-Hour at a pressure of 50 pascals \\
AHU & air handling unit \\
AMY & actual meteorological year \\
& American Society of Heating, Refrigeration and Air-Conditioning \\
ASHRAE & Engineers \\
BC & base case \\
BED & Building Energy Data-book \\
BES & building energy simulation \\
Bhp & brake horsepower \\
BIM & building information modelling \\
BLC & building load coefficient \\
BMS & building management systems \\
BPS & building performance simulation \\
CB & Canal Building \\
CDD & cooling degree days \\
CESAR & Canadian Energy Systems Analysis Research \\
CFSR & climate forecast system reanalysis \\
CFM & cubic feet per minute \\
CH4 & methane \\
CHP & central heating plant \\
CO 2 e & carbon dioxide equivalent \\
CCHP & combined cooling, heat and power \\
COP & coefficient of performance \\
CSS & Cascading Style Sheets \\
CSV & comma separated value \\
CTFs & conduction transfer functions \\
CV(RMSE) & coefficient of variation of the root mean square error \\
CWEC & Canadian Weather for Energy Calculations \\
D3 & Data-Driven Documents \\
DCI & Digital Campus Innovation \\
EnergyPlus (E+) & whole building energy simulation program \\
ECMs & energy conservation measures \\
ECO's & energy conservation opportunities \\
EIA & Energy Information Administration \\
EMS & energy management system \\
EUI & energy usage intensity \\
FEMP & Federal Energy Management Program \\
&
\end{tabular}




\begin{tabular}{|c|c|}
\hline FMP & Facilities Management and Planning \\
\hline GenOpt & Generic Optimization Program \\
\hline GWh & gigawatt hour \\
\hline GBS & green building studio \\
\hline gbXML & Green Building XML schema \\
\hline GHGs & greenhouse gas emissions \\
\hline GJ & gigajoules \\
\hline Gpm & US gallons per minute \\
\hline GWP & global warming potential \\
\hline $\mathrm{HCI}$ & Human Computer Interaction \\
\hline HDD & heating degree days \\
\hline HP & horsepower \\
\hline HRW & heat recovery wheel \\
\hline HTML & Hypertext Markup Language \\
\hline HVAC & heating, ventilation, and air conditioning \\
\hline IEA & International Energy Agency \\
\hline IESO & Independent Electricity System Operator \\
\hline IFC & Industry Foundation Class \\
\hline IPMVP & International Performance Measurement and Verification Protocol \\
\hline IQRs & interquartile ranges \\
\hline jQuery & JavaScript library \\
\hline $\mathrm{L} / \mathrm{s}$ & liters per second \\
\hline $\mathrm{Lbs} / \mathrm{hr}$ & pounds per hour \\
\hline LCA & life cycle assessment \\
\hline MBE & mean bias error \\
\hline MEP & mechanical, electrical, and plumbing \\
\hline MFA & material flow analysis \\
\hline MWh & megawatt hour \\
\hline MW & megawatt \\
\hline $\mathrm{N}_{2} \mathrm{O}$ & nitrous oxide \\
\hline NECB & National Energy Code of Canada for Buildings \\
\hline NWRC & Natural Wildlife Research \\
\hline NRCan & Natural Resources Canada \\
\hline O\&M & operation and maintenance \\
\hline PHP & Hypertext Preprocessor \\
\hline PJ & petajoules \\
\hline PSTAR & primary and secondary term analysis and renormalization \\
\hline RMSE & root mean square error \\
\hline Rpm & revolutions per minute \\
\hline SHGC & solar heat gain coefficient \\
\hline
\end{tabular}




$\begin{array}{ll}\text { SVG } & \text { Scalable Vector Graphics } \\ \text { T\&D } & \text { transmission and distribution } \\ \text { TRNSYS } & \text { TRaNsient SYstems Simulation Program } \\ \text { UM } & \text { urban metabolism } \\ \text { VAV } & \text { variable air volume } \\ \text { VFD } & \text { variable frequency drive } \\ \text { VSD } & \text { variable-speed drive } \\ \text { WWR } & \text { window to wall ratio }\end{array}$

\begin{tabular}{|c|c|}
\hline Symbols & Full Name \\
\hline$\overline{\beta \text { motor }}$ & fraction of heat loss from motor to air stream \\
\hline$C_{p}$ & specific heat capacity $\left(\mathrm{kJ} / \mathrm{kg}{ }^{\circ} \mathrm{C}\right)$ \\
\hline CDW & condensing water \\
\hline CHW & chilled water \\
\hline $\mathrm{CO}_{2}$ & carbon dioxide (ppm) \\
\hline$E_{\text {emb, elect, (water) }}$ & $\begin{array}{l}\text { total electrical energy embedded for pumping and treatment of } \\
\text { water including losses }(\mathrm{kWh})\end{array}$ \\
\hline$E_{\text {emb, elect, (wastewater) }}$ & $\begin{array}{l}\text { total electrical energy embedded for pumping and treatment of } \\
\text { wastewater including losses }(\mathrm{kWh})\end{array}$ \\
\hline EA & exhaust air \\
\hline$\sum F$ & friction losses by fans, pumps, and air filters (kWh) \\
\hline$f$ & $\begin{array}{l}\text { factor determined based on the location of the fan in the air } \\
\text { stream }\end{array}$ \\
\hline$h$ & enthalpy $(\mathrm{kJ} / \mathrm{kg})$ \\
\hline$h_{d a}$ & specific enthalpy for dry air \\
\hline$h_{g}$ & specific enthalpy saturated water vapor \\
\hline HW & hot water-glycol \\
\hline IC & influence coefficient \\
\hline IP Base case & input values of the base case \\
\hline$\Delta I P$ & changes in input values from the base case \\
\hline$L$ & $\begin{array}{l}\text { losses in the system (e.g. frictional losses through AHU ducts } \\
\text { and heat transfer across the walls of the AHU) }\end{array}$ \\
\hline$\dot{m}$ & mass flow rate $(\mathrm{kg} / \mathrm{s})$ \\
\hline$\dot{m}_{\text {condensation }}$ & amount of vapor condensate from latent cooling $(\mathrm{kg} / \mathrm{s})$ (tonnes) \\
\hline$\dot{m}_{\text {net exfiltration }}$ & $\begin{array}{l}\text { amount of net exfiltration leaving the building envelope }(\mathrm{kg} / \mathrm{s}) \\
\text { (tonnes) }\end{array}$ \\
\hline$\dot{m}_{\text {internal water vapor added }}$ & $\begin{array}{l}\text { amount of internal water vapor added by occupants, equipment, } \\
\text { and infiltration }(\mathrm{kg} / \mathrm{s}) \text { (tonnes) }\end{array}$ \\
\hline MA & mixed air \\
\hline$\eta$ & efficiency \\
\hline
\end{tabular}


$n$

$\mathrm{OA}$

OAT

$\% O A$

OP Base case

$\triangle O P$

$\rho$

$p$

$P_{e l}$

$\triangle P$

$Q$

$Q_{\text {cooling coils }}$

$Q$ cooling tower

Qheating coils

$Q_{L}$

$\dot{Q}_{\text {motor }}$

$\dot{Q}_{\text {motor-to-air }}$

Qrecovery

$Q_{\text {recovery,sensible }}$

$Q$ recovery,latent

$Q_{\text {steam }}$

$Q_{\text {pred }, i}$

$Q_{\text {data }, i}$

$\bar{Q}_{\text {data }, i}$

$Q_{\text {cam, water }}$

RA

reference

$S$

SA

$T$

U-value

$W$

$W_{\text {fan }}$ number of data samples obtained from sensors or sub-meters

outdoor air

outdoor air temperature

outdoor air fraction

output values of the base case

changes in output values from the base case

density $\left(\mathrm{kg} / \mathrm{m}^{3}\right)$

correction parameter

electric energy consumption (kWh)

differential pressure $(\mathrm{kPa})$

thermal load (kWh)

amount of energy absorbed by the cooling coils (kWh)

amount of energy extracted by the cooling towers $(\mathrm{kWh})$

amount of energy added by heating coil $(\mathrm{kWh})$

amount of energy gained from the chiller evaporator $(\mathrm{kWh})$

rate of heat addition from the fan to the air stream due to

frictional losses $(\mathrm{kWh})$

rate of heat addition from the fan to the air stream due to

frictional losses based on the fan location in the air stream $(\mathrm{kWh})$

instantaneous recovered energy by the heat recovery wheel

$(\mathrm{kWh})$

sensible energy recovered by energy wheel $(\mathrm{kWh})$

latent energy recovered by energy wheel

amount of thermal energy added by steam $(\mathrm{kWh})$

predicted value during the $\mathrm{i}^{\text {th }}$ period

measured value during the $\mathrm{i}^{\text {th }}$ period

measured average during the period

the total campus water consumption $\left(\mathrm{m}^{3}\right)$

return air

this subscript" is used to refer to the type of fluid passing

through the pumps (i.e. steam, hot water (HW), chilled water

(CHW), or condensate water (CDW))

number of data points obtained from sensors or sub-meters that

contain errors (such as missing or inconsistent data points)

supply air

temperature $\left({ }^{\circ} \mathrm{C}\right)$

overall heat transfer coefficient $\left(\mathrm{W} / \mathrm{m}^{2} . \mathrm{K}\right)$

humidity ratio $\left(\mathrm{kg} / \mathrm{kg}_{\text {dry }}\right.$ air $)$

work done by the fan $(\mathrm{kWh})$ 


\section{Chapter 1: Introduction}

\subsection{Background}

Our environment currently faces serious challenges, including urbanization (transportation, buildings, bridges, wastewater, etc.), rapid fossil fuel depletion, climate change, increased concentration of air pollutants, unsustainable fresh water use, greenhouse gas emissions (GHGs), and limited land-surface resources [1]. Moreover, the significant expansion in population typically leads to an increase in energy use, GHG emissions, and electricity usage [2].

Ten percent of Canadians work, study, or live on campuses [3]. A large number of facilities and building functions are found on the campus level. Each function as described by Klein-Banai et al. [4] acts as small communities (e.g., residences, library, academic, research, laboratories, transportation, and agricultural activities), having an aggregate environmental impact like small towns. In Ontario, about $12.5 \%$ of the $384.9 \mathrm{PJ}$ (petajoules or $10^{15}$ joules) that are allocated to commercial and institutional (C\&I) buildings is used for educational facilities. University campuses in Ontario recorded the second highest energy intensity consumption of $1.78 \mathrm{GJ} / \mathrm{m}^{2}$ (or $\sim 500 \mathrm{kWh} / \mathrm{m}^{2}$ ) compared to other provinces [5]. GHG emissions for Ontario was estimated to be 170.2 megatonnes of carbon dioxide equivalent $\left(\mathrm{CO}_{2} \mathrm{e}\right)$ in 2014, out of which 18.13 megatonnes, 2.27 megatonnes, and 0.89 megatonnes $\left(83.8 \mathrm{~kg} \mathrm{CO} 2 \mathrm{e} / \mathrm{m}^{2}\right)$ were allocated for the $\mathrm{C} \& \mathrm{I}$ sector, educational facilities, and universities, respectively [5, 6]. Canada's current target is to reduce GHGs by 30 percent below 2005 levels by 2030, which means an absolute reduction of 200 megatonnes [7]. 
Nowadays, modern commercial buildings' (i.e., buildings that are not residential or industrial) resource consumption is metered at various levels of spatial and temporal resolution to track and reduce GHG emissions. This leads to having many data sources at the building level. As a result, utility data (such as that obtained from sensors and submeters) are available, but this does not translate to an abundancy information (such as energy flows across different building/system components). Since the data are frequently obtained from multiple systems and technologies of varying vintages, the metering and data logging systems are often inconvenient and difficult to access. Moreover, some data cannot be obtained because of the high cost of meters. For instance, it is currently not cost effective to meter standard office equipment power consumption for each room.

Utilizing Building Performance Simulation (BPS) combined with calibrated building models could help in estimating unmeasured energy flows, such as solar gains, heat loss from infiltration, etc. In order to use BPS models to help in understanding the thermal behavior of an existing buildings, it should be calibrated with measured data.

Furthermore, building information modelling (BIM) concept has emerged as a powerful technology that involves the generation management of digital representations of physical and functional characteristics of building [8]. Utilizing BIM in building performance analysis can facilitate a more accurate and efficient analysis process. However, in order to perform a successful BIM-based building performance analysis, it is necessary to improve the interoperability between a BIM based architectural model and energy analysis programs [9].

In the context of Carleton University in Ottawa, Canada, the current tools for 
obtaining and visualizing data are discussed. Facilities Management and Planning (FMP) department on campus utilizes PowerLogic $\AA$ IONEEM, an enterprise energy management software developed by Schneider as shown in Figure 1.1. This software enables FMP to obtain temporal resolution (i.e. monthly, daily, and hourly) of metered data for electricity, natural gas, steam, and water for each building. This tool can provide a comparison of the consumption of each building over a specific time. Energy and water bills are provided by the local utilities, but these are only at monthly resolution and may fail to identify inefficiencies and operational problems. It should be noted that Carleton University is among the more advanced campuses with regards to building energy and controls infrastructure.

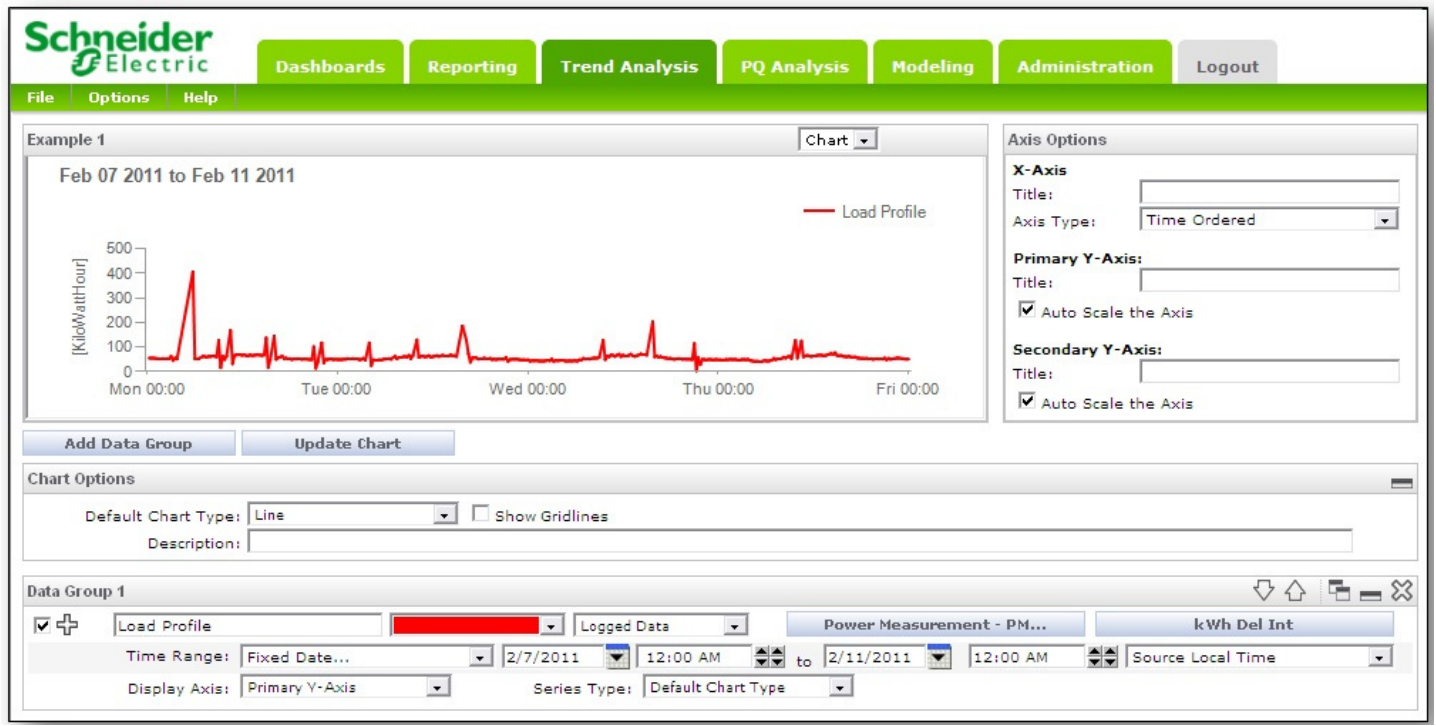

Figure 1.1: IONEEM interface

On the building level, some of the buildings on campus utilizes building management system (BMS) by a building controls contractor to monitor the energy consumption by different sources. Energy consumption data are obtained from sensors and sub-meters installed in the building operated by several different companies. These data 
are accessible through enteliWEB, a web-based application developed by Delta Controls as shown in Figure 1.2. This tool enables the user to obtain temporal resolution of energy consumption by different sources (such as chillers, lighting, plug loads, fans, elevators, etc.). Moreover, this tool also provides other data (such as air handling unit (AHU) supply temperature, supply and return air flows, operational schedules for different components, etc.).

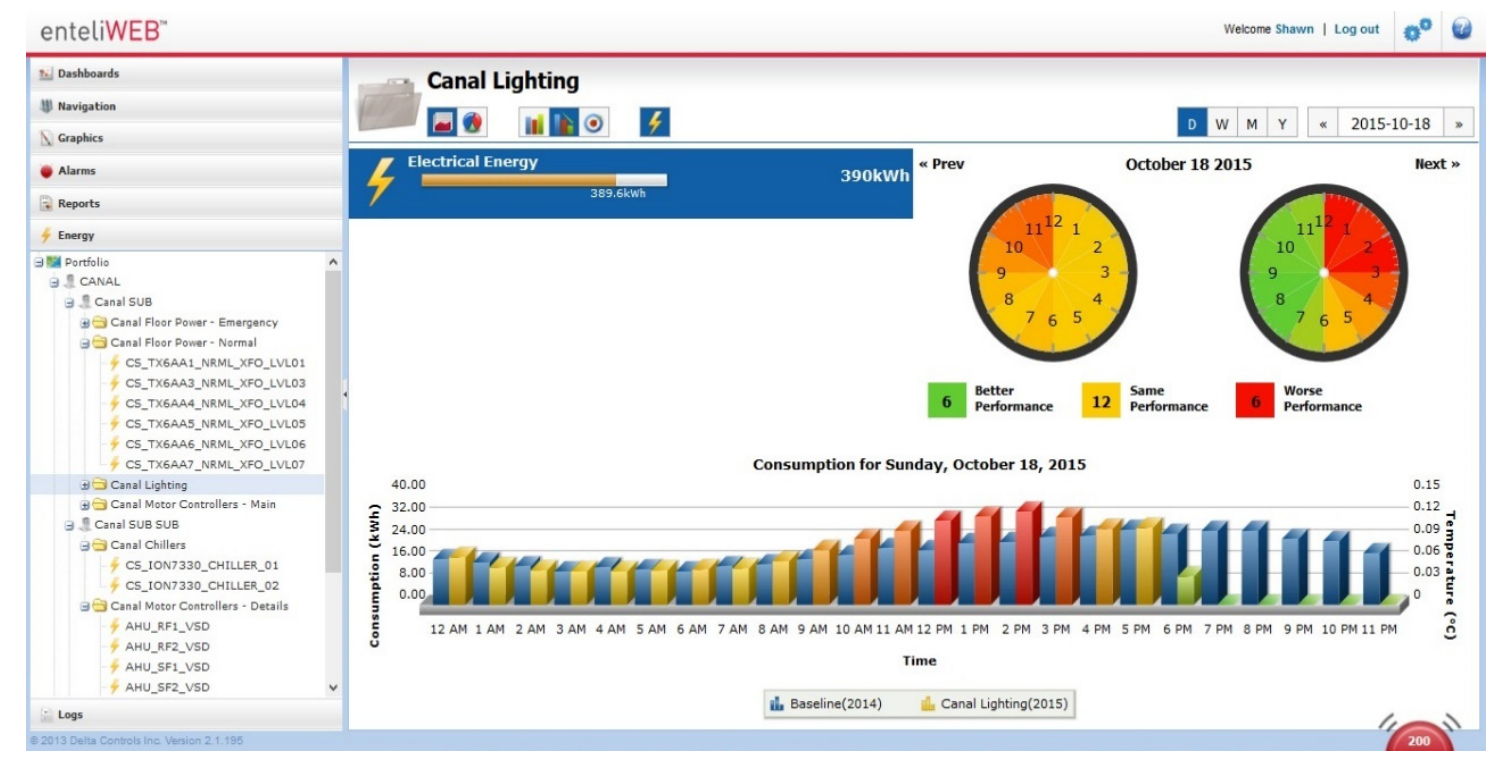

Figure 1.2: enteliWEB interface

The aim of the above-mentioned example is to illustrate the state-of-art of tools for obtaining and visualizing data targeting building operators. The current tools can provide a quantitative analysis of the consumption of each building and different building systems. However, the existing tools are limited in accounting for losses due to inefficiency of systems or distribution losses through network of pipes. Moreover, the current visualization tools typically do not provide a comprehensive understanding of how each component affects the whole system performance, including upstream and downstream building systems and environmental impacts. Furthermore, these tools are limited in 
tracking underperforming buildings and visualizing overall campus performance. These tools do not readily identify opportunities for energy savings on different scales.

The most common visualization techniques used to visualize building's resource consumption are 1) binning plots, 2) carpet plots, 3) voxel-plot, 4) superimposed line graph plot, 5) line chart, 6) bar graph, and 7) color mapping. Moreover, many researchers claimed that conveying simulation results in the form of tables and graphs is not useful to nonexpert designers in the field of building science [10,11,12]. An especially effective method to visualize of materials and energy flows that have existed for over 100 years is the Sankey diagram. Sankey diagrams could lend themselves well to building energy applications, however they are seldom used. Sankey diagrams can provide relative flow magnitudes, direction of flows, inputs and outputs of interacting systems, energy recovery, and spatial representation (e.g., the layout systems and components can be approximately laid out in a Sankey diagram). Figure 1.3 shows an example of utilizing Sankey diagrams to visualize energy flows from source to end-use on campus level. 


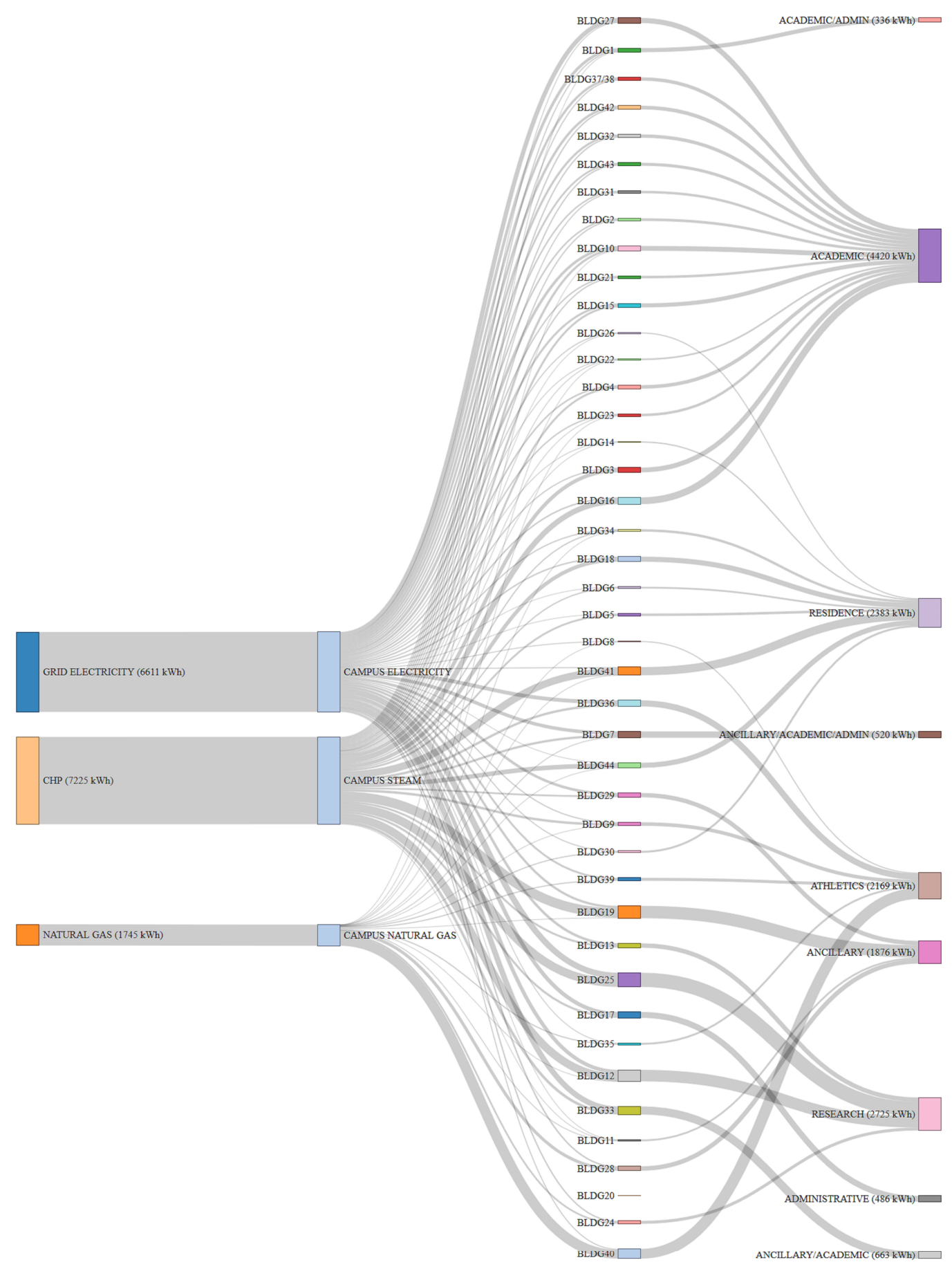

Figure 1.3: Sankey diagrams showing energy supply and demand on campus level 


\subsection{Motivation and Objectives}

Modern commercial buildings are equipped with abundant sensors and sub-meters to track and reduce energy consumption and the associated GHGs. This could yield significant data; however, these data are not necessarily in a readily comprehensible format that are aimed at key stakeholders (operators, owners, technicians). Moreover, some data cannot be obtained from sensors or sub-meters such as solar gains, infiltration and ventilation rates, etc. Calibrated BPS models could help estimate unmeasured energy flows and provide a greater understanding on how each building component is performing.

Utilization of BIM in building performance analysis can facilitate a more accurate and efficient analysis process. Moreover, using BIM in building operations is useful for record keeping and maintenance. However, there is a lack of utilizing this application by building operators.

The current data availability and visualization tools have some limitations in identifying system inefficiencies and possible solutions. Furthermore, these tools are limited in visualizing overall campus performance and providing qualitative analysis. Furthermore, conveying simulation results in the form of tables and graphs is not useful to non-expert designers in the field of building science.

The main objective of this research is to seek better accounting, tracking, and visualization methods to understand the opportunities to reduce GHG emissions and water and energy use in the built environment. The study utilizes different spatial (i.e. campus, building-level, and HVAC system level) and temporal (i.e. annual, monthly, and hourly) resolutions. The objective is to improve accessibility of these data to all stakeholders, 
including building operators, planners, occupants, and utilities. This thesis aims to make operational problems more visible and quantifiable. The main purpose of this work is the ability to generalize the methods, tools and findings to other campuses or communities and buildings. Moreover, the research aims to develop a new visualization technique to identify possible inefficiencies and inform operators on building component/system performance. Furthermore, the developed visualization technique should also be used to understand the upstream and downstream impact to evaluate various design iterations and facilitate the decision-making by designers and engineers during early design stages.

Key objectives of this thesis include:

1) Develop methods to analyze and visualize energy, water, natural gas, utility cost, and GHG emissions data at the building and campus scale using Sankey diagrams and other graphical methods. Moreover, the research aims to normalize resource consumption and GHG emissions per unit area and per occupant bases.

2) Develop a method to analyze energy flow and the associated cost by using measured data (obtained from BMS) and model data (obtained from calibrated BPS model).

3) Develop a method to analyze and visualize energy and mass flows and the corresponding energy cost through different components of a typical air handling unit (AHU) and plant loops of commercial buildings using Sankey diagrams. The aim of the proposed method is to convert sparse data obtained from sub-meters and sensors to estimated HVAC energy, and mass flows by using first principles. 
4) Develop a framework to automate the process of creating Sankey diagrams on the building-level and HVAC system level from energy simulation outputs (EnergyPlus).

\subsection{Research Methodology}

This work is divided into two main parts. The first part "Developing Methods to Enhance Campus Operations" focuses on developing a comprehensive energy use assessment at different scales by using a combination of measured data and models that could be generalized to other existing modern commercial buildings and campuses to facilitate the decision-making by building operators. Figure 1.4 shows the overall framework of the research utilizing spatial and temporal analysis. This work involves the application and testing on Carleton University campus and its Canal Building. This ensures that the theory is grounded in practicality and it also allows the usability to be tested on real stakeholders (building operators, campus planners, and accountants). 

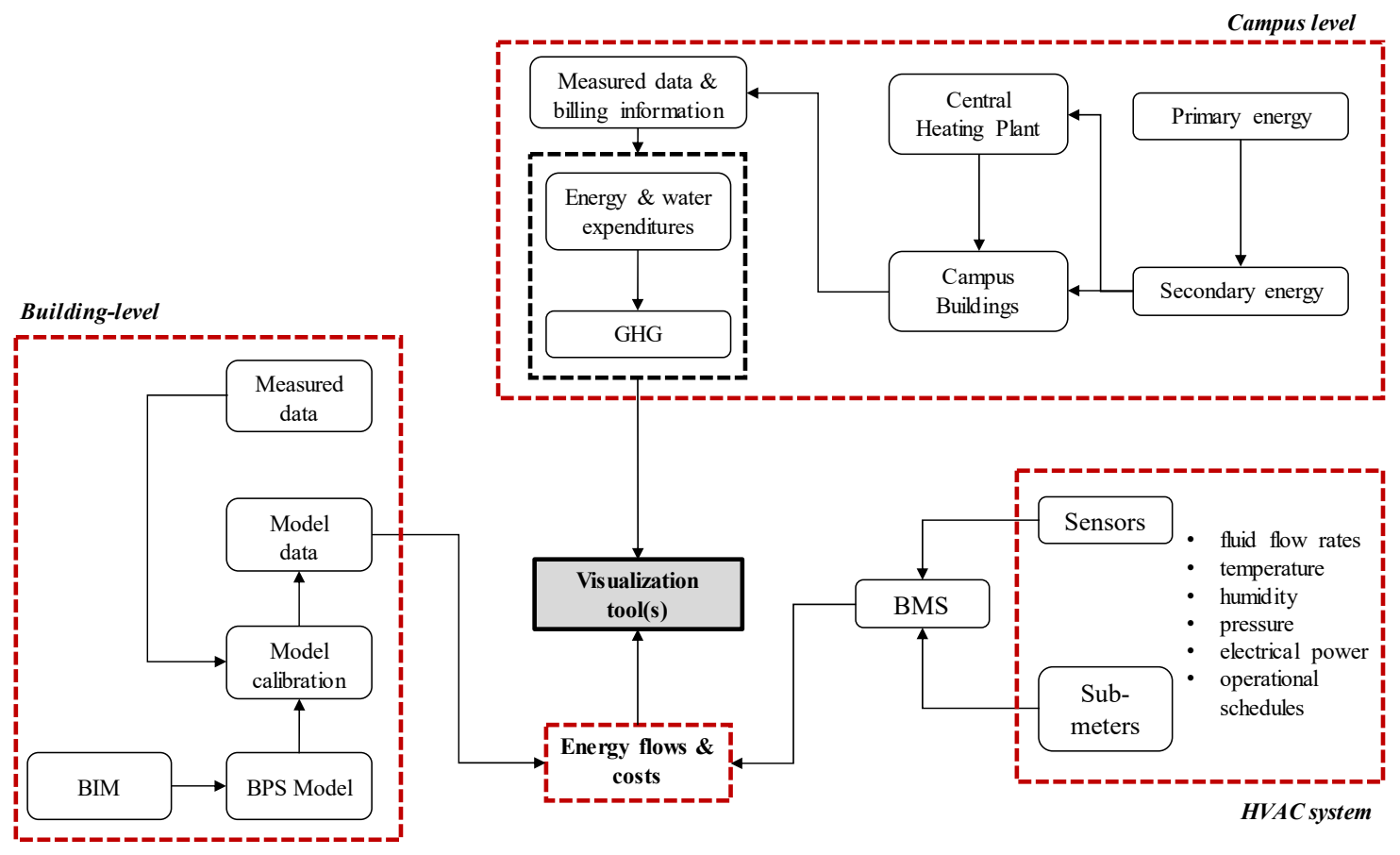

Figure 1.4: Flowchart showing the overall framework to analyze and visualize energy flows, GHGs, and the corresponding energy cost on different scales (campus, building, and HVAC)

On the campus level, the research focuses on developing methods to analyze and visualize building-level water, natural gas, and electricity consumption and the upstream environmental impacts. The aim of the proposed methods is to seek better accounting, tracking, and visualization methods to understand the opportunities to reduce GHG emissions and water and energy use. The methodology focused on: 1) converting measured data into energy, natural gas, and water consumption by the main power plant and by different buildings on campus, 2) converting primary to secondary energy including generation, transmission, and distribution processes, 3) normalizing resource consumption per unit area and per person, and 4) estimating equivalent carbon dioxide emissions.

On the building-level, the research proposes an integrated framework to estimate and visualize energy flows and the corresponding energy cost on the building level to 
provide a comprehensive energy use assessment that could be generalized to other existing modern commercial buildings to facilitate the decision-making by building operators. The framework consists of 1) develop BIM model, 2) a method to convert BIM to BPS, and 3) a method to calibrate BPS models. EnergyPlus was selected as the BPS tool due to its technical documentation and versatility and capability of simulating complex building systems.

On the HVAC system level, the research focuses on converting sparse data obtained from sensors and sub-meters into estimated energy and mass flows in addition to the corresponding cost through different components of heating, ventilation, and airconditioning (HVAC) system using Sankey diagrams.

The second part of this work "Implementation of Sankey Diagrams as A DecisionMaking Tool" aims to investigates the feasibility of using Sankey diagrams to visualize and understand the upstream and downstream performance impacts of building design decisions. The aim of this research is to utilize Sankey diagrams to visualize building energy performance on the building-level and building system level (HVAC system) obtained from simulation outputs. This research proposes a workflow to obtain, analyze, and visualize energy flows obtained from simulation outputs. This work involves the application and testing of Sankey diagrams on large office reference building models that comply with the National Energy Code of Canada for Buildings (NECB). This part also provides a framework to automate the process of creating Sankey diagrams from simulation (EnergyPlus) outputs through a user-graphical interface web page. Hypertext Preprocessor (PHP), JavaScript, and Hypertext Markup Language (HTML) codes are used to develop the user-graphical interface web page. 


\subsection{Document Structure}

Chapter 2 provides a critical review on the current methodologies that measure campus resource consumption, production of waste, and GHG emissions through various accounting and visualization techniques. The research then tackles the interoperability between BIM based architectural model and building performance analysis tools. A review of the state-of-art of different calibration approaches is then presented. The chapter then provides a review of the state-of-art of the current visualization techniques used for building energy monitoring and parametric design of buildings. This chapter also demonstrates the concept of Sankey diagrams.

The thesis is then divided into two main parts. Part one "Developing Methods to Enhance Campus Operations" consists of three chapters. Each chapter is published or submitted as journal article providing a critical review of the existing methods and the description of the developed framework. Moreover, each chapter implements the developed framework on a selected case study, followed by results and discussion section.

Chapter 3 (published in Energy and Buildings journal: Visualization of Energy, Water Consumption and GHG Emissions: A case study of university campus) focuses on providing literature review on methodologies (i.e. urban metabolism, material flow analysis, life cycle analysis, and ecological footprints) that measure campus resource consumption, production of waste, and GHG emissions. Moreover, current data availability and visualization techniques on the campus level are also discussed. The proposed methods are then discussed. The proposed methods are then applied to Carleton University, a 45building comprehensive Canadian university campus. This work is also followed by a survey applied to building operators on Carleton University campus on evaluating Sankey 
diagrams for tracking building/campus energy and water use.

Chapter 4 (submitted to Automation in Construction journal: Data Visualization and Analysis of Energy Flow on a Multi-Zone Building Scale) provides literature review on the utilization of BPS and BIM in the field of building operations. The current data availability and visualization techniques on the building-level are also discussed. The developed framework is then discussed. The proposed method is then applied to a large Canadian university building (The Canal building at Carleton). Finally, an online survey is applied to a sample group to assess usability and effectiveness of visualizing energy flow and the associated cost using Sankey diagrams on the building level.

Chapter 5 (in review at Energy and Buildings journal: Development of Sankey Diagrams to Visualize Real HVAC Performance) provides literature review on the current data availability and visualization techniques on the HVAC system level. The proposed framework is then discussed and applied to one of the air handling units (AHUs) in the Canal building (the same case study used in the previous chapter).

The second part "Implementation of Sankey Diagrams as A Decision-Making Tool" consists of two chapters. The first chapter (chapter 6) is a conference article titled "Visualization of Building Performance using Sankey Diagrams to Enhance the DecisionMaking Process", the Symposium on Simulation for Architecture and Urban Design (SimAUD) conference. This chapter includes a literature review on the current visualization techniques used for parametric design of buildings. The Sankey diagrams developed in the first part on the building-level and HVAC system level are applied to a large office reference building model that comply with the National Energy Code of 
Canada for Buildings (NECB). The aim of this work is to investigate the feasibility of utilizing Sankey diagrams in visualizing energy flows obtained from energy simulation tool outputs on different spatial resolution: building-level and building system level (HVAC system). This chapter also discusses the technical challenges of creating Sankey diagrams from simulation data. The second chapter (chapter 7) titled "Automating the Creation of Sankey Diagrams" provides a framework to automate the process of creating Sankey diagrams from simulation outputs (EnergyPlus). This framework involves the development of a user-graphical interface web page using Hypertext Preprocessor (PHP), JavaScript, and Hypertext Markup Language (HTML). The developed framework is then applied to different office (i.e. large, medium, and small) reference building models that complies with the National Energy Code of Canada for Buildings (NECB) in order to test different building systems.

Conclusion, findings, and future work for the whole thesis is provided in Chapter 8. The developed methods could yield greater insights about opportunities for energy savings and inspect operational problems on various scales. Moreover, the developed methods could also facilitate the decision making in developing the requirements of some building/system component in the energy efficiency code for commercial buildings. The information contained in the developed Sankey diagrams would be useful in informing policy and investment decisions related to energy use patterns on the campus, buildinglevel, and HVAC system level. 


\section{Chapter 2: Literature Review}

The scope of this work focuses on developing a comprehensive energy use assessment at different scales (from campus to building system level) by using a combination of measured data and models. The objective of this research is to provide a useful workflow and set of methods to facilitate the decision-making by building operators, campus planners, design engineers, architects, and other stakeholders. Figure 2.1 shows the overall methodology of the research. The framework consists of: 1) campus metabolism, 2) measured data synthesis, 3) interoperability between BIM and energy analysis tools, 4) model calibration, and 5) visualization techniques. These are briefly reviewed below in order to frame the current methodology. More thorough literature review is provided in each chapter.

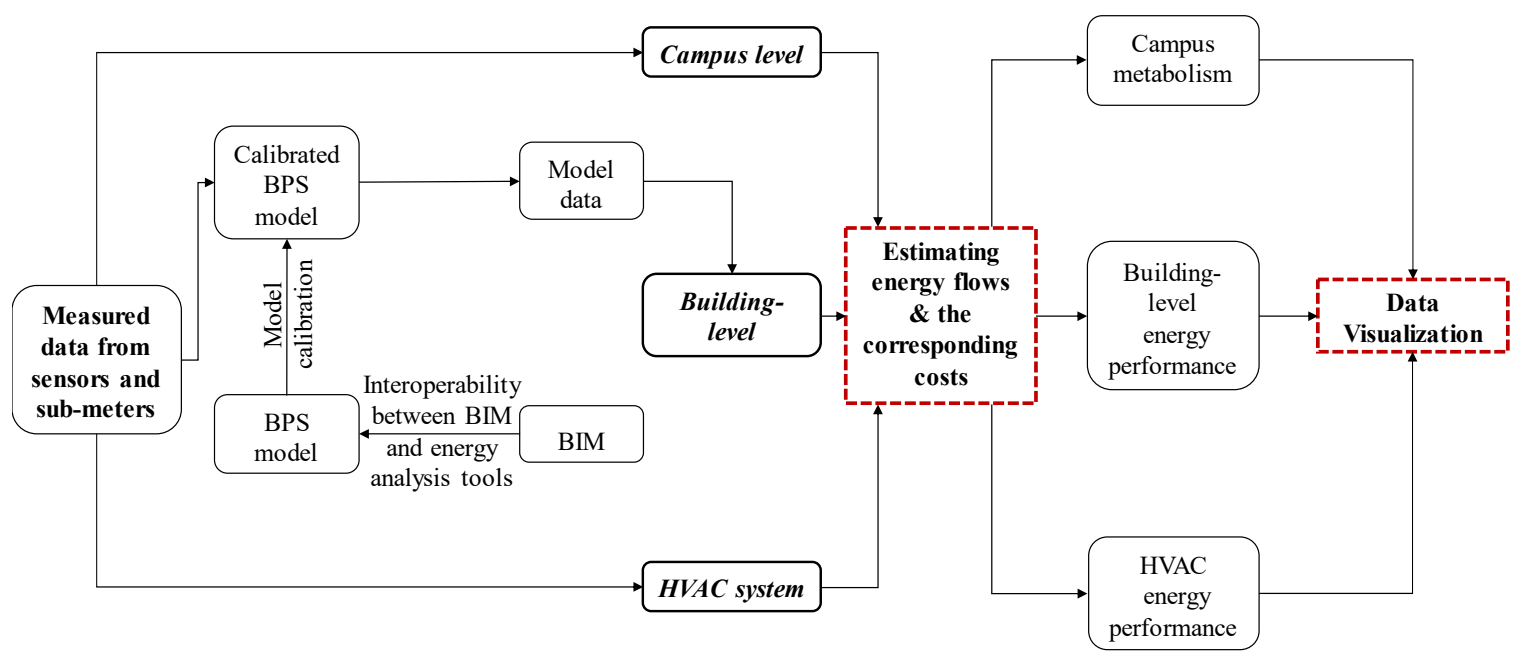

Figure 2.1: The proposed methodology to estimate and visualize energy flows obtained from sensors and sub-meters on campus, building-level, and HVAC system

\subsection{Campus Metabolism}

Many researchers have developed methodologies that measure campus resource 
consumption, production of waste, and GHG emissions through various accounting and visualization techniques $[13,14]$. Key concepts and methods of analysis that have emerged are urban metabolism, material flow analysis, life cycle analysis, and ecological footprints. These methods are discussed in detail in Section 3.2.

\subsection{Measured Data Synthesis}

Resource use can be measured from an entire campus down to an individual building, from a building system or interior space, or even at the scale of an occupant's end-use load. As metering infrastructure is deployed at greater densities, more spatially and temporally resolved data become available [15]. Advanced metering may provide data at hour- or minute-long intervals at a full building scale, while sub-metering is capable of providing data at near continuous time resolution and at a sub-building scale. Sub-metered data can be used to provide feedback on energy consumption to building operators or building users to promote behavioral change that leads to energy conservation [16]. This refined timescale provides insight into daily, weekly, or seasonal operation and maintenance $(\mathrm{O} \& \mathrm{M})$ issues, occupant behaviors, performance of installed equipment (e.g., HVAC and lighting), and verification of installed efficiency technologies. The availability of sub-metering data provided at more discrete levels can be used by automation systems as part of fault detection, thereby identifying problems with installed equipment [17].

Sub-meters and other sensors installed in a building should be connected to building management systems (BMS). The data points are transmitted via communication networks and analyzed for the use by O\&M staff and occupants, as well as by facilities managers, possibly in remote locations [18]. Such coordination is especially important in complex commercial and multi-unit residential buildings, in which many systems overlap, and 
decisions affecting operating characteristics [19]. These sensing and control technologies separate the decision-making into smaller elements.

For electrical systems, meters can be installed to track whole-building energy use, sub-panel energy use (e.g., lighting, plug loads, etc.), or a specific end use (e.g., a motor, or a chiller). For water, steam, and natural gas, meters are typically installed in respect with the distribution lines. The four predominant levels of resource metering are one-time/spot measurement, run-time measurement, short-term measurement, and long-term measurement [20]. One-time or spot measurement applications are useful in many "baseline" activities to understand instantaneous energy use, equipment performance, or loading. In addition, they can measure changes in consumption following energy efficiency upgrades. Run-time measurements are often used to establish hours of operation for devices or systems as part of reducing energy consumption through shortened operation times. Short-term monitoring combines both duration of operation with energy consumption to establish a time-series record of energy or resources used. Long-term monitoring also makes use of duration of operation and energy consumption, though the equipment used is often installed on a permanent basis, and measurements are taken at a prescribed frequency and resolution to meet the established energy monitoring requirements [21].

\subsection{Interoperability between BIM and Energy Analysis Tools}

Building Information Modeling (BIM) is one of the most promising developments in the architecture, engineering and construction (AEC) industries [22]. With BIM technology, an accurate virtual model of a building is constructed digitally. BIM can be defined as a modeling technology and associated set of processes to produce, communicate, and analyze building models [23]. BIM technology can support and improve many business 
practices. Although, the AEC/FM (Facility Management) industry is in the early days of BIM use, significant improvements have already been realized (compared to traditional 2D CAD or paper - based practices). BIM have the potential to provide pre-construction benefits to owner, design benefits, construction and fabrication benefits, and post construction benefits [24]. BIM also presents an important opportunity to support the creation of building performance simulation (BPS) models that are used for building operations [23]. BPS tools require input of building surface information, material properties, system description, lighting and plug loads, and operational schedules. Interoperability between BIM and BPS should minimize data repetition and inherent human error, and enable rapid iterations of a design. BIM platforms can transfer data to energy analysis tools by translating the BIM data model to a format needed by the analysis tool [25]. The following discussion includes BIM protocols and interoperability between BIM and energy analysis tools.

\subsubsection{BIM Protocols}

The most common BIM protocols used to exchange data between BIM and energy analysis tools are Industry Foundation Class (IFC) and Green Building XML schema (gbXML). The goal of IFC is to provide a universal basis for process improvement and information sharing in the construction and facilities management industries [26, 27]. The IFC format is able to provide geometric information, non-geometric properties (material properties) and relationship between the components. However, the IFC data exchanged by commercial tools is general and it includes generic data, thus most of the specific information will be missed in the exchange process $[28,29]$.

The gbXML (green building XML schema), developed by Green Building Studio 
(GBS), facilitates the exchange of data among BIM and energy analysis programs [30]. The gbXML schema allows exchanging some HVAC information that is missed in the IFC schema, but nevertheless this format is not mature enough and it is limited to simple designs given that the exportation process is not able to read complex geometries [31].

\subsubsection{Interoperability between BIM and EnergyPlus}

The aim of this section is to provide background on data exchange between BIM and energy analysis tool (EnergyPlus in this case). BIM modelling tools have different data exchange support abilities. BIM tools such as Revit Architecture could provide detailed building geometry information. While, Revit MEP support additional information such as building construction, space and zone, material properties, operational schedules, equipment and lighting power densities [9].

BIM shows its capability in exporting complex geometries and detailed information of each zone and building component. However, obtaining a very detailed energy model is not practical as it might cause system crashes, errors and long simulation time [32]. Thus, it is crucial to carefully select model resolution based on the scope of the study. EnergyPlus recommended the following to reduce the simulation time [33]: 1) reduce number of windows by lumping similar windows on surface, 2) group zones having same boundary conditions, HVAC system, or internal gains schedules, 3) exclude semi-exterior unconditioned zones from the model and replace them with an equivalent R-Value, and 4) exclude structural elements.

A study by Moon et al. [9] evaluated the interoperability between a BIM based model and building performance analysis programs. Based on the evaluation results, it 
showed that EnergyPlus was able to import building geometry information including surfaces (floor, wall, roof), openings (window, door) from the BIM based model. However, EnergyPlus showed some problems in converting building geometry such as the location of the openings. Thus, a validation function of building geometry is required before running simulation. It is also recommended that for EnergyPlus, a graphical interface should be required to import gbXML data, such as (Designbuilder, GBS, or Open Studio). In terms of space composition, space definition in gbXML is composed of "Space" and "Zone". The space includes information such as internal loads, operation schedules and HVAC set point temperature, which is used for HVAC sizing. EnegyPlus is capable of converting each space to zone, by which post-processing task is required to group zones having same HVAC system, internal gains, and boundary conditions. For building construction, gbXML is capable of translating information such as materials and layers to EnergyPlus. However, material properties (i.e. U-value and SHGC) and layers for window types are not exported to gbXML. Internal loads (such as people, lighting, and equipment) and operational schedules could be exported to gbXML and translated to EnergyPlus. On the other side, HVAC system information in gbXML produced by Revit cannot be imported to EnergyPlus, as GBS is used to graphically model detailed HVAC system.

\subsection{Model Calibration}

The aim of the model calibration in the proposed framework is to obtain greater data about building performance and to supplement measurements. The following sections briefly discuss existing building energy simulation models, state-of-art calibration methods, selection of calibration parameters, and accuracy of calibrated building performance simulation. 


\subsubsection{Background}

Since the 1960s, building energy simulation has emerged as an attempt to emulate reality and improve on traditional manual methods to study and optimize the energy performance of buildings and systems [34, 35]. Initially, building performance simulation (BPS) models were mainly used for design purposes [36]. More recently, the area of application of BPS models was extended in further (post-construction) stages of the building life cycle, such as building operation optimization [37], technical and economical evaluation of Energy Conservation Measures (ECMs), commissioning and functional performance testing [38], fault detection and diagnosis [39], building energy management and energy audit [40]. At the same time, graphical user interfaces were developed to facilitate use of such complex tools [41].

More recently, with the development of modern simulation tools, it appeared that whole-building simulation might help at all the levels of an energy efficiency service process, from inspection audit to last retrofit and on-going commissioning actions [42]. In order to use BPS models to help in understanding the thermal behavior of an existing situation requires the BPS model to be able to closely represent the actual behavior of the building under study. However, discrepancies could occur between energy model and measured data due to lack of information about building's construction quality, occupant behavior, actual materials/equipment used, deterioration of building systems, and infiltration, etc. Thus, a model calibration is needed when utilizing BPS on different levels of analysis (inspection/audit, evaluation of energy conservation opportunities (ECO's) or continuous performance analysis).

The fitting of a BPS model to an existing situation involves using as-built 
information, survey observations and short and/or long term monitoring data to iteratively adjust the parameters of the BPS model. Data such as building envelope characteristics or the type of HVAC system are easily identified, but many parameters such as actual ventilation and infiltration flow rates and actual use of lighting and appliances, have to be properly adjusted.

\subsubsection{Existing Building Energy Simulation Models}

Depending on the type of the building (residential, school, healthcare...), the model must account for various conditions and attention must be paid to the level of detail required to perform the desired analysis [40].

There are two main categories that should be differentiated; inverse and forward modeling techniques. Inverse models are generated based on measured data and generally rely on regression analysis $[43,44,45]$ to deduce representative building parameters (building load coefficient (BLC), base load, and building time constant) as shown in Figure 2.2. Reddy et al. [45] proposed an inverse model parameter to estimate building and ventilation parameters from non-intrusive monitoring of heating and cooling energy use. It appears that the identification process is accurate when daily data over an entire year are used to perform calibration of this model. In the frame of the ASHRAE RP-1050 research project and in relation with the ASHRAE Guideline 14-2002, Kissock et al. [44] developed an inverse linear calculation toolkit for the purpose of measuring energy savings. The toolkit includes the algorithms necessary to find the best fit for three, four and fiveparameters change-point models and to evaluate the uncertainty of model predictions. 


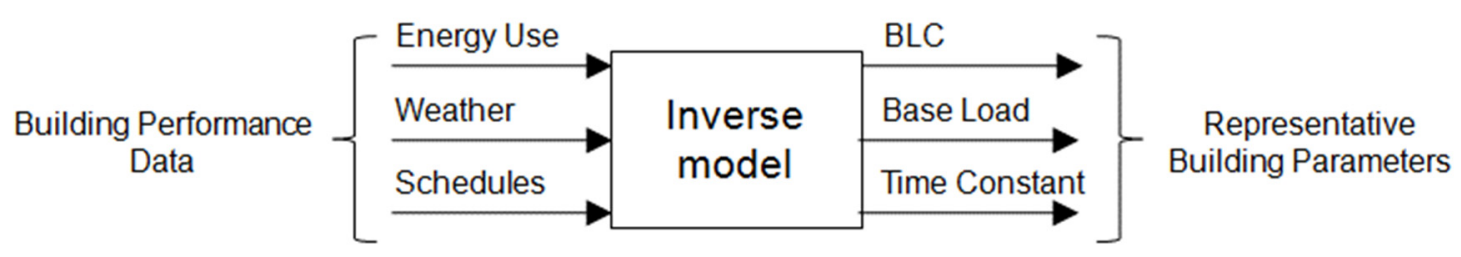

Figure 2.2: Inverse modeling (Adapted from Krarti [40])

Forward modeling involves using physical models that is able to predict the future of a system described by some parameters (geometry, location, and nominal performances) as shown in Figure 2.3. The calibration of such models implies an iterative tuning process of the parameters of the model to match recorded data. The most commonly used forward building energy simulation platforms are DOE-2 [46], TRNSYS [47] and EnergyPlus [33].

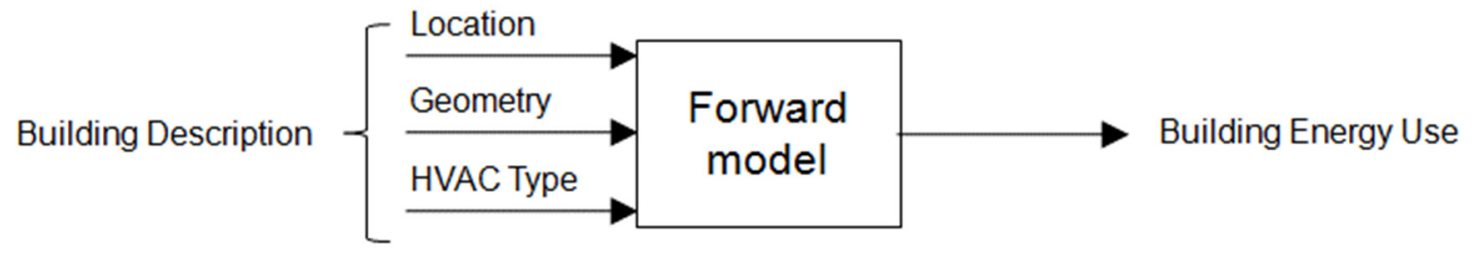

Figure 2.3: Forward modeling (Adapted from Krarti [40])

Although the inverse models are generally simpler than the forward approach, their flexibility is limited by the representative building parameters used to formulate the model and the accuracy of the recorded data used to calibrate the model. For instance, it is not possible to evaluate the impact of replacing an existing chiller by a more efficient one if no parameter of the inverse model addresses chiller performance. On the other side, forward models are more flexible and rely on physical representations, which can be adapted to various situations. Moreover, these models can be continuously updated to consider additional influences (e.g. developing/implementing a more detailed chiller model to take into account the improvement of part load performance due to the replacement of the 
chiller).

\subsubsection{Previous Work and Existing Calibration Methodologies}

The calibration of a forward building energy simulation program involves numerous input parameters as common building energy data is a highly under-determined problem that would result in a non-unique solution [48]. Kaplan et al. [49] stated that it would never be possible to identify the exact solution to the calibration problem. Furthermore, the sensitivity issues may be of primary importance in the calibration field. Another constraint relies on the fact that calibration requires a dynamic matching over one year between computed and measured values and not a static one at one condition [50]. These elements make the calibration of building energy simulation models challenging.

The most common calibration methods can be classified as follows [35, 51]:

- Manual iterative calibration based on the user's experience and consisting of an adjustment of inputs and parameters on a trial-and-error basis until the simulation output matches the measured data;

- Calibration based on specific graphical representations and comparative displays of the results to orient the calibration process;

- Calibration based on special tests and analytical procedures involving specific intrusive tests and measurements, such as the PSTAR (Primary and Secondary Term Analysis and Renormalization) method [52];

- Analytical and mathematical calibration methods involving the use of optimization algorithms.

The above methods are not exclusive and could be coupled (e.g. use of graphical 
and statistical analysis methods to support iterative manual calibration, semi-automatic procedures coupling mathematical and heuristic manual methods).

1) Manual calibration method:

This method is the most commonly used. Numerous authors and practitioners use this kind of methods to adjust the parameters of detailed BPS models. However, these methods are highly dependent on the user's experience and rarely applied in a systematic way. Furthermore, it is based on an ad-hoc approach in which the analyst manually tunes the parameters until a solution is obtained. Kaplan et al. [49] presented a methodology to evaluate the ECO's (Energy Conservation Opportunities) implemented in a monitored building. This work is one of the first successful calibrations of a detailed simulation model. Other examples of manual calibration could be found in the work by [53-61].

The calibration framework proposed by Reddy et al. [62], urges the analyst to refrain from searching for an optimal solution and instead be satisfied with several plausible ones. Further, Oliva [63] pointed out that a good fit to the data is not a sufficient condition when calibrating a model. Moreover, Oliva claimed that the estimated parameters should be explicitly considered.

\section{2) Graphical and Statistical Methods}

This method includes techniques based on graphical representations and comparative displays of the results. They generally consist of time-series and scatter plots. Two main techniques can be listed for their wide application: 3D comparative plots and calibration and characteristics signature.

3D comparative plots have been developed to analyze hourly differences between 
simulated and measured data during the whole simulation period [64]. This method is used for calibrating time-dependent parameters such as schedule loads. Hourly values are computed and compared in the plot. This type of representation has also been used with statistical indices (MBE and CV(RMSE)) for analyzing the goodness-of-fit of the building model.

The term signature is used to refer to a graphical representation of the difference between the simulated and the measured energy performance of a particular case study [65]. The "calibration signature" describes a normalized plot of the difference between measured energy consumption and the corresponding simulated values (residual) as a function of (e.g. outdoor air temperature). Such a normalized residual analysis depending on the outdoor temperature is more useful for determining errors in the simulation than a simple scatter plot between measured and calculated values.

On the other side, "characteristic signature" describes the residuals between two simulation results of different input values versus (e.g. outdoor air temperature). The difference between two simulations shows a typical pattern to the outdoor temperature. This method could help the user to record changes of simulation results according to the input change.

When assessing both characteristic and calibration signatures, the differences between the two curves help users to detect errors in the simulation inputs for calibrating the model. It is thus possible to study the effect of the input parameters variation in the building models looking at the calculated signature. A proposed methodology based on the use of the calibration and characteristic signatures is presented in the work done by Liu et 
al. [65]. Other models and methods could be found in the work by [66-70].

Methods combining manual iterations and graphical/statistical tools seem to be well adapted to energy audit purposes. These methods are very flexible and could be adapted to most cases. Moreover, these methods allow the auditor identifying, visualizing and inspecting the behavior of the building under study during the calibration process. However, attention should be paid to keep the calibration method systematic and reproducible and to integrate sensitivity issues [71]. Furthermore, the graphical method relies on a trial and error process, which requires skilled personnel and extensive analyses.

\section{3) Calibration Based on Analytical Procedures}

This category is based on analytical and test procedures such as short or long-term monitoring periods. This method does not employ mathematical or statistical procedure for the calibration process. Measurement tests (such as blower door tests or wall thermal transmittance measures) are considered for calibrating the building models. However, as they are quite intrusive, especially when buildings are constantly occupied, they cannot always be performed.

Short-term monitoring and inspections can also assist the calibration process. For example, the PSTAR (Primary and Secondary Term Analysis and Renormalization) method [52] is a unified method of hourly simulations of a building and analysis of performance data based on the use of short-term monitoring data.

The building energy balance is assessed as sum of the heat flows calculated after the audit inspection. Heat flows are assessed based on macro-dynamic calculations. Each heat flow term is then classified as primary or secondary depending on its magnitude. 
Primary terms are then renormalized (calibrated) based on monitored data. Moreover, audit reports are also included in this calibration method [72].

\section{4) Automated Calibration Methods}

This method includes all approaches that cannot be considered user driven and are built on sort of automated procedures [73]. They can be based on mathematical procedures (e.g., Bayesian calibration) or analytical approaches.

Bayesian analysis is a statistical method that employs probability theory to compute a posterior distribution for unknown parameters. It is used for calibration purposes for incorporating directly uncertainties in the process $[74,75]$. Recently different studies $[76$, $77,78]$ have focused on the application of this technique to the building simulation domain.

Based on the Bayesian theory [79], a set of values of the uncertain parameters of the energy model is formulated in order to find a matching between the simulation outcomes and the measured data. Three different sources of uncertainty are investigated: parameter uncertainty in the energy model, discrepancy between the energy model and the real building behavior, and observation error. A prior probability density function is assigned to each calibration uncertain parameter based on users' judgment and experience. The energy model outputs are denoted as normal distribution. In order to solve the multivariate distribution, the Markov Chain Monte Carlo algorithm is used to compute the probability density function of the calibration parameters considered. Finally, a posterior distributions function of each uncertain parameter is assessed.

Meta-model, another mathematical function, which determines coefficients, based 
on a limited number of input/output combinations. Different meta-model techniques can be found in literature [80]: polynomial regression (PR), multivariate adaptive regression splines (MARS), kriging (KR), radial basis function networks (RBF), and sigmoidal neural networks (NN). A meta-model can be defined as a "model of a model" [81] or a surrogate model that is usually used for reducing the model complexity. For instance, meta-models created within building simulation programs are based on an essential characterization of the building. This type of building energy models is defined by varying all of the input parameters within a certain range, around its baseline design. Usually for creating an $n$ sample of the $p$ inputs, sampling techniques like in the Monte Carlo Analysis is used [72]. Once the meta-model is derived from the model, an optimization algorithm is applied. One of the main benefits of meta-model is the reduced simulation time that allow different optimization scenarios to be performed. Meta-model is also employed as sensitivity analysis for the assessment of the building energy performance.

The term optimization is used in building simulation to refer to an automated approach based on numerical simulation and mathematical optimization [82, 83]. Optimization-based methods are usually built on the coupling between a building simulation software (e.g., EnergyPlus, TRNSYS, etc.) and an optimization program (e.g., GenOpt), which employs optimization algorithms [83, 84]. Simulation-based optimization has recently been used for various applications in building simulation [85-88], and also for the calibration of building models [89-95]. In order to perform the optimization, an objective function has to be set within the optimization program. Usually in calibration application, the objective function is defined as a function of the difference between measured and simulated data. The optimization is thus based on the matching between a 
set of measured data and simulated data.

There are some limitations in integrating such methods with local/additional measurements issues yielding the calibration process not to be reproducible and flexible. Moreover, some parameters (as schedules) cannot be easily adjusted during the automated calibration process. The optimization method considered to be a black box process, where the user enters some free parameters and the objective function. Furthermore, the optimization methods do not provide the user with a deeper understanding of which parameters significantly affect the simulation. Another limitation is that the optimization may produce mathematically correct but physically meaningless results. Therefore, the user needs to countercheck results before using them.

\subsubsection{Selection of Calibration Parameters}

A large number of input data are always involved in the building modeling process. However, the quantity may vary depending on the level of detail pursued in the model definition and on the data availability (e.g., problems of data quality). Measured data is sometimes used for providing the model with further information (e.g., building occupancy, temperature set point, etc.) during validation of the calibrated model based on statistical indices. Furthermore, not all input data affect the investigated energy consumption in the same ways. Thus, it is important to identify throughout a screening analysis the parameters that influence most the building model and define their level of uncertainty [72].

Uncertainty and sensitivity analyses represent an integral part of the modeling process, especially for calibrated simulation. It also could help overcoming gaps in the 
building knowledge, identifying and ranking the sources of uncertainties [96].

As stated by Fabrizio and Monetti [72], even when the building model is created upon the "best plausible estimates", in terms of input parameters values and building system and operation definition, disagreements between simulated and measured energy consumption may be encountered. Such discrepancies may be attributed to an incomplete knowledge of the building. The building model may thus not correctly reflect the real behavior of the building intended to be simulated. In the building physics domain, uncertainties may result from different sources. Heo [74] identified four main categories of uncertainty sources in building models, when carrying out studies on energy retrofit analyses as shown in Table 2.1.

Table 2.1: Source of uncertainty in building energy models [74]

\begin{tabular}{|l|l|}
\hline \multicolumn{1}{|c|}{ Category } & \multicolumn{1}{c|}{ Factors } \\
\hline Scenario uncertainty & - Outdoor weather conditions \\
& - Building usage/occupancy schedule \\
\hline \multirow{3}{*}{ Building physical/operational uncertainty } & - Building envelope properties \\
& - Internal gains \\
& - Operation and control settings \\
\hline \multirow{3}{*}{ Model inadequacy } & - Modeling assumptions \\
& - Simplification in model algorithm \\
\hline Observation error & - Ignored phenomena in the algorithm \\
\hline
\end{tabular}

The first category "Scenario uncertainty" focuses on the external environment (e.g., outdoor weather conditions) and the building use. In most cases, actual weather data are used for creating real weather file to be employed in simulation. Incomplete and fragmented data can determine uncertainties in the data collection and consequently in the definition of the actual weather data. On the other side, uncertainties can affect the 
definition of the building use, which is set by means of schedules expressing the building occupancy and operation. The second category refers to uncertainties in the building modeling, with special regards to the building envelope thermo-physical properties, internal gains (people, appliances, lightings, etc.), HVAC definition and its operational and control settings, and ventilation and infiltration rates. The third category concerns uncertainties in the building model as physical representation of the real phenomena. Each building model is an approximation of a real building, created on the basis of assumptions and simplifications. The last category refers to observation errors in the measured data. The data quality of measurement used for calibrating the model can affect the accuracy of the results. Therefore, uncertainties in measured data have to be taken into account.

\subsubsection{Accuracy of Calibrated BPS Models}

A calibration process consists of adjusting the parameters of a model through iterations until the model predictions agree to an acceptable level of accuracy with the recorded data within some predefined criteria. The definition of these criteria is a complex issue and it is difficult to determine how close a tolerance needs to be to fulfill the calibration objective [49].

It is recommended using Mean Bias Error (MBE), Root Mean Square Error (RMSE) and Coefficient of Variation of the Root Mean Square Error CV(RMSE) to evaluate calibration accuracy as shown in Eq. (2.1), (2.2), and (2.3), respectively [53, 56, 97].

$$
M B E=\frac{\sum_{i=1}^{n}\left(Q_{p r e d, i}-Q_{\text {data }, i}\right)}{(n-p) \times \bar{Q}_{\text {data }}}
$$




$$
\begin{gathered}
R M S E=\sqrt{\frac{\sum_{i=1}^{n}\left(Q_{\text {pred }, i}-Q_{\text {data }, i}\right)^{2}}{n}} \\
C V(R M S E)=\frac{R M S E}{Q_{\text {data }}}=\frac{\sqrt{\frac{\sum_{i=1}^{n}\left(Q_{\text {pred }, i}-Q_{\text {data }, i}\right)^{2}}{n-p}}}{\bar{Q}_{\text {data }}}
\end{gathered}
$$

where

$Q_{\text {pred,i }}$ is the predicted value during the $\mathrm{i}^{\text {th }}$ period

$Q_{\text {data,i }}$ is the measured value during the $\mathrm{i}^{\text {th }}$ period

$\bar{Q}_{\text {data }, i}$ is the measured average during the period

$n$ is the number of available data points (or periods)

$p$ is a correction parameter

The common Mean Bias Error (MBE) approach is an important measure of calibration but it is limited when dealing with large compensating errors (i.e. positive and negative errors) could lead to a zero MBE [56]. Thus, using CV(RMSE) in addition to MBE to describe the variability of the results allows preventing this compensation problem. For calibrated simulations, it is suggested that the MBE and CV(RMSE) indices should be used with $p=0$ and $p=1$, respectively [50]. Reddy and Maor [50] justified this choice by the particularities of the calibration problem.

The three standards dealing with calibration are ASHRAE 14-2002 [97], IPMVP (International Performance Measurement and Verification Protocol) [98], and FEMP (Federal Energy Management Program) [99]. These standards also provide numerical criteria as shown in Table 2.2 to calibrate BPS models to building energy use data. The 
values proposed by ASHRAE Guideline 14-2002 and FEMP are the same but very different from the ones proposed by IPMVP.

Table 2.2: Calibration tolerances by different standards

\begin{tabular}{|c|c|c|c|}
\hline Index & ASHRAE 14-2002 & IPMVP & FEMP \\
\hline MBE (monthly) & $\pm 5 \%$ & $\pm 20 \%$ & $\pm 5 \%$ \\
\hline CVRMSE (monthly) & $\pm 15 \%$ & $\pm 5 \%$ & $\pm 15 \%$ \\
\hline MBE (hourly) & $\pm 10 \%$ & & \\
\hline CVRMSE (hourly) & $\pm 30 \%$ & & \\
\hline
\end{tabular}

Kaplan et al. [49] proposed different tuning tolerances instead of a unique set of tolerances as shown in Table 2.3. The criteria proposed depends on the energy uses (lighting, cooling, heating, fan, etc.) and tuning periods (monthly, daily, hot period, and cold period).

Table 2.3: End-use specific tolerances (adapted from Kaplan et al. [49])

\begin{tabular}{|c|c|c|c|}
\hline End-Use & Tuning Period & $\begin{array}{c}\text { Monthly End-Use } \\
\text { Tolerances }\end{array}$ & $\begin{array}{c}\text { Day-type Profile } \\
\text { Tolerances }\end{array}$ \\
\hline Indoor lighting & All & $\pm 5 \%$ & $\pm 15 \%$ \\
\hline Outdoor lighting & All & $\pm 5 \%$ & $\pm 15 \%$ \\
\hline DHW & All & $\pm 5 \%$ & $\pm 15 \%$ \\
\hline Plug loads & All & $\pm 5 \%$ & $\pm 15 \%$ \\
\hline Heating & Winter & $\pm 15 \%$ & $\pm 25 \%$ \\
\hline Cooling & Summer & $\pm 15 \%$ & $\pm 25 \%$ \\
\hline Ventilation fans & All & $\pm 15 \%$ & $\pm 25 \%$ \\
\hline Whole-building & All & $\pm 10 \%$ & $\pm 15 \%$ \\
\hline
\end{tabular}

Statistical indices should not be the unique way to evaluate the accuracy of the calibration [100] and could lead to a "blind" calibration missing numerous influences (weather, occupancy, operation, etc.). Furthermore, even if the "net effect" of all the "knobs" yields to a simulated output close to the measured one, there is no guarantee that all individual "knobs" are properly tuned. 


\subsection{Visualization Techniques}

The aim of this section is to provide a background on the current available building energy dashboard tools on various scales. Visualization techniques used for parametric design of buildings are also discussed in this section. This section also demonstrates the concept of Sankey diagrams. Moreover, methods of Evaluating Visualization Techniques are discussed as this study is followed by as survey to test the effectiveness and usability of Sankey diagrams in visualizing energy flows and the corresponding costs on various scales.

\subsubsection{Current Building Energy Visualization Tools}

There are various tools to visualize building's resource consumption. Pulse Energy and Building Dashboard are examples of visualization tools that shows real-time building consumption of energy, natural gas, hot water, chilled water, and steam. More visualization tools on campus, building-level, and building system level are discussed in Section 3.2, Section 4.2., and Section 5.2, respectively.

\subsubsection{Current Visualization Techniques used for Parametric Design of Buildings}

Nowadays, several visualization techniques have been developed to visualize energy simulation output results. The most common visualization techniques used for parametric design of buildings are 1) interactive parallel coordinates plot, 2) carpet plots, 3) voxel-plot, 4) superimposed line graph plot, 5) line chart, 6) bar graph, and 7) color mapping plots. More discussion is provided in Section 6.2. However, Pratt and Bosworth [10] claimed that an effective visualization method is needed when inspecting a high order (more than three variables) search space. Moreover, many researchers claimed that conveying simulation results in the form of tables and graphs is not useful to non-expert 
designers in the field of building science $[10,11,12]$. Furthermore, the above-mentioned visualization methods have some limitations in evaluating simulation results in relation to non-performative or qualitative analysis [10]. Moreover, the current visualization tools typically do not provide a comprehensive understanding of how each component affects the whole system performance, including upstream and downstream building systems. For instance, the choice of light bulbs/fixtures should incorporate their impact on heating and cooling loads and costs. This research attempts to evaluate different visualization techniques used in BPS tools as shown in Section 2.5.4.

\subsubsection{Sankey Diagrams}

An especially effective method to visualize materials and energy flows that have existed for over 100 years is the Sankey diagram [101]. Sankey diagrams are comprised of arrows of varying widths, where the width indicates relative magnitude of flow and the direction indicates the connection between sources and sinks for each flow. Sankey diagrams allow resource flows to be visualized within complex systems with interacting subsystems. They are particularly useful for understanding relative flows of resources and identifying opportunities for which waste outputs could be recirculated as inputs back into the system.

There are various applications of using Sankey diagrams in visualizing energy flows. Sankey diagrams can be applied both for visualizing performance of existing systems as well as during design using modelling and simulation. Literature reviews on utilizing Sankey diagrams to visualize energy flows on campus, building-level, and building system level (HVAC system) are discussed in Section 3.2, Section 4.2, Section 5.2, respectively. Recently, the International Energy Agency (IEA) utilized Sankey 
diagrams to visualize energy balance through different processes and resource consumption by different sectors for various countries [102]. Furthermore, Canadian Energy Systems Analysis Research (CESAR) utilized Sankey diagrams to visualize national energy supply, demand, and exports as shown in Figure 2.4 [103]. This shows the importance of Sankey diagrams as a decision-making tool on different context.

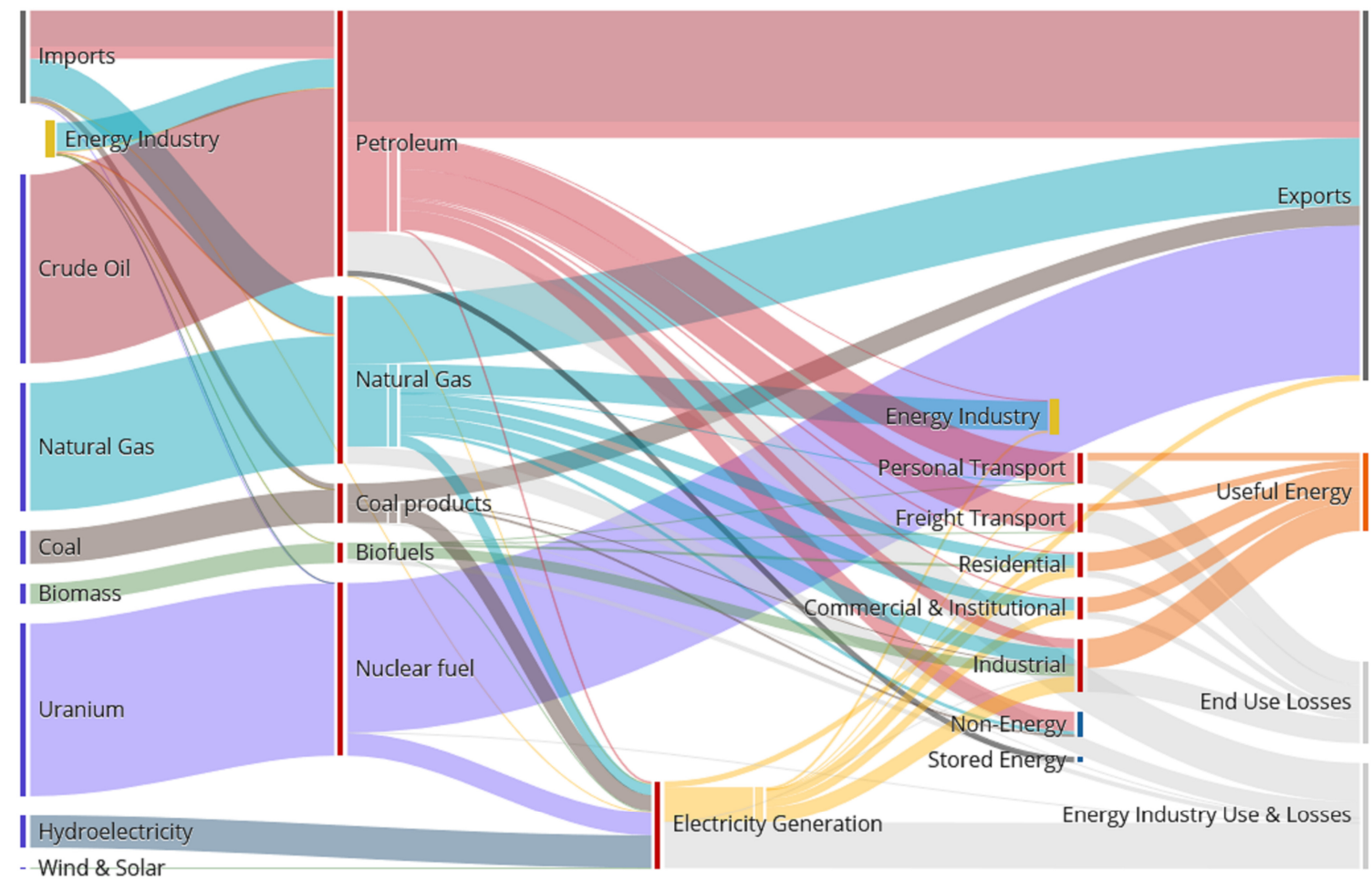

Figure 2.4: visualization of national energy supply, demand, and exports using Sankey diagrams. Source [103].

\subsubsection{Methods for Evaluating Visualization Techniques}

Visualization methods is divided into two high-level categories: scientific visualization and information visualization. The scientific visualization refers to visual representation of physically based objects, such as buildings, human body, and components in a building. While, information visualization focuses on data or information, which is non-physically based, such as building components and sensor readings which aims to 
improve human knowledge and to identify trends or patterns $[104,105]$.

In the field of building operations, it is essential that building operators understand the dynamics of energy use, control strategies, and occupant comfort in buildings. Performance data for a building are valuable at all stages of its life cycle and design, commissioning, and operation. However, transforming raw data obtained from sensors and sub-meters into useful information for utilities and energy service companies to operate their buildings optimally is no small task [106]. The purpose of the data analysis at different timescales is to identify or verify the energy savings achieved by energy-efficient technology or identifying malfunctions in building equipment. Effective data visualization depends on designing graphic presentation formats that clearly reveal technical phenomena relevant to the performance of the building.

Data obtained from BMS are increasingly valuable in energy performance contracting, utility demand-side management program evaluation, and other activities where regulatory requirements or financial contracts require documenting actual energy performance and costs [106]. Data visualization alone cannot improve a building's performance and must be integrated to an operation and maintenance $(\mathrm{O} \& \mathrm{M})$ plan to be effective.

There has been much research into the proper methods of evaluating the efficiency, effectiveness and user satisfaction (i.e. building operators) of visualization tools [107-110] . Ferwerda et al. [111] suggested that visualization can be evaluated based on the following criteria: 1) the degree of simplicity that the visualization can provide to the users to understand and complete their tasks; 2) whether the visualization follows simple rules 
of perception and design; 3) how realistic the visualization resembles the real-world objects; and 4) whether the visualization allows users to uncover something new in a dataset. Lakowski and Plaissant [112] split the evaluation of visualization systems into three levels: the component, system, and work levels. The evaluation of the effectiveness of visualization is difficult due to the complex multidisciplinary nature of visualization. However, recent discussion in the Human Computer Interaction (HCI) community, Greenberg and Buxton [110] and North [113] suggested the removal of benchmark tasks as it leaves a little room for deep and qualitative insights. The following discussion focuses on evaluating different visualization techniques used in BPS tools based on the criteria suggested by [111] as shown in Table 2.4 .

Table 2.4: Comparison between different visualization techniques used in BPS tools

\begin{tabular}{|c|c|c|c|c|}
\hline \multirow[b]{2}{*}{$\begin{array}{l}\text { Visualization } \\
\text { tools used in } \\
\text { BPS tools }\end{array}$} & \multicolumn{4}{|c|}{ Evaluation of visualization techniques } \\
\hline & $\begin{array}{l}\text { Degree of } \\
\text { simplicity }\end{array}$ & $\begin{array}{c}\text { Allows users to } \\
\text { complete their } \\
\text { tasks }\end{array}$ & $\begin{array}{l}\text { Resembles } \\
\text { objects in BPS } \\
\quad \text { tools }\end{array}$ & $\begin{array}{l}\text { Allows users to } \\
\text { uncover } \\
\text { something new } \\
\text { in a dataset }\end{array}$ \\
\hline \multirow[b]{2}{*}{$\begin{array}{l}\text { Interactive } \\
\text { parallel } \\
\text { coordinates } \\
\text { plot }\end{array}$} & 2 & 2 & 1 & 3 \\
\hline & \multicolumn{4}{|c|}{$\begin{array}{l}\text { Strengths: Provide quantitative analysis to allow various design iterations } \\
\text { to be evaluated by the designers [114]. } \\
\text { Weaknesses: Can show one-dimensional relation between axes. Moreover, } \\
\text { the user can arrange the axes (parameters) in a unique order based on the } \\
\text { analysis. This requires the use of heuristics and experimentation [115]. } \\
\text { Moreover, it becomes difficult to interpret the data with large sets of data. }\end{array}$} \\
\hline \multirow[b]{2}{*}{ Contour plots } & 1 & 2 & 1 & 3 \\
\hline & \multicolumn{4}{|c|}{$\begin{array}{l}\text { Strengths: Provide good quantitative analysis if the dataset is highly } \\
\text { dependent on two variables [116]. } \\
\text { Weaknesses: Limited in providing quantitative analysis for multi-variable } \\
\text { datasets [10]. They are also less intuitive to use [117]. }\end{array}$} \\
\hline \multirow[b]{2}{*}{ 3D Voxel-plots } & 2 & 3 & 2 & 3 \\
\hline & \multicolumn{4}{|c|}{$\begin{array}{l}\text { Strengths: Provide quantitative analysis to evaluate different design } \\
\text { alternatives, with a maximum of three dimensions [10]. } \\
\text { Weaknesses: Provide only three-dimensional search space and cannot }\end{array}$} \\
\hline
\end{tabular}




\begin{tabular}{|c|c|c|c|c|}
\hline \multirow[b]{3}{*}{$\begin{array}{l}\text { Superimposed } \\
\text { line graph plots }\end{array}$} & \multicolumn{4}{|c|}{ incorporate more dimensions in the analysis [10]. } \\
\hline & 3 & 3 & 2 & 3 \\
\hline & \multicolumn{4}{|c|}{$\begin{array}{l}\text { Strengths: Provide quantitative analysis to evaluate different design } \\
\text { alternatives on multi-dimensional parameters [10]. } \\
\text { Weaknesses: Are more complex to interpret the displayed information } \\
{[10] .}\end{array}$} \\
\hline \multirow[b]{2}{*}{$\begin{array}{l}\text { Color mapping } \\
\text { plots }\end{array}$} & 2 & 3 & 2 & 3 \\
\hline & \multicolumn{4}{|c|}{$\begin{array}{l}\text { Strengths: Provide quantitative analysis if the data set is highly dependent } \\
\text { on two variables [117]. } \\
\text { Weaknesses: Are less quantitative analysis compared to contour plots as the } \\
\text { analysis is based on colors which is hard to interpret by the user. Thus, it } \\
\text { requires carefully designed colormaps [117]. }\end{array}$} \\
\hline \multirow[b]{2}{*}{$\begin{array}{l}\text { Line chart } \\
\text { Bar graph } \\
\text { Scatter plots }\end{array}$} & 2 & 3 & 2 & 3 \\
\hline & \multicolumn{4}{|c|}{$\begin{array}{l}\text { Strengths: Are useful in providing quantitative analysis to inspect trends and } \\
\text { patterns over time [11]. } \\
\text { Weaknesses: Cannot be easily interpreted by non-expert designers. The } \\
\text { complexity of the representation increases notably with the dimension [12]. }\end{array}$} \\
\hline
\end{tabular}


Part I: Developing Methods to Enhance Campus Operations 


\section{Chapter 3: Visualization of Energy, Water Consumption and GHG Emissions}

This chapter was previously published as:

(Abdelalim A., O'Brien W. and Shi Z., "Visualization of Energy and Water Consumption and GHG Emissions: A Case Study of a Canadian University Campus," Energy and Buildings, vol. doi:10.1016/j.enbuild.2015.09.058, 2015.)

It is reprinted here under the terms of the copyright license agreement with Elsevier B.V. The copyright license agreement is provided in Appendix G.

This chapter focuses on developing methods to analyze and visualize campus and building-level water, natural gas, and electricity consumption and the upstream environmental impacts. Minor changes have been made to Section 3.2 "in the second paragraph". These changes include the amount of energy use and GHG emissions by commercial and instructional buildings and educational facilities in Ontario. Values and units in Table 3.2 have been updated.

\subsection{Abstract}

Campuses, communities, and other building clusters are major users of energy and water and thus can have a significant environmental impact. Frequently, buildings' resource consumption is tracked at various levels of spatial and temporal resolution through meters, in order to monitor and reduce resource consumption. However, the metering and data logging systems are often inconvenient and difficult to access due to use of multiple systems and technologies of varying vintages. This research proposes several methods to analyze and visualize building-level water, natural gas, and electricity consumption and the upstream environmental impacts: Sankey diagrams and bar charts that normalize metered 
values by floor area and occupancy. The objective is to improve accessibility of these data to all stakeholders, including building operators, planners, occupants, and utilities. The methods are then applied to a 45-building Canadian university campus and an array of graphical representations of the data are provided. The resulting analysis and visualization reveals significant variation in consumption between buildings regardless of building vintage and function. Furthermore, it is concluded that identifying resource consumption reducing strategies, once inefficient buildings have been identified, would require higher data resolution - both spatial and temporal.

\subsection{Introduction}

Our environment currently faces serious challenges, including urbanization (transportation, buildings, bridges, wastewater, etc.), rapid fossil fuel depletion, climate change, increased concentration of air pollutants, unsustainable fresh water use, greenhouse gas emissions (GHGs), and limited land-surface resources [1]. Moreover, the significant expansion in population leads to an increase in energy flows, GHG emissions, and electricity usage [2].

A large number of facilities and building functions are found on the campus level; each function as described by Klein-Banai et al. [4] acts as small communities (e.g., residences, library, academic, research, laboratories, transportation, and agricultural activities), having an aggregate environmental impact like small towns. In Ontario, about $12.5 \%$ of the $384.9 \mathrm{PJ}$ (petajoules or $10^{15}$ joules) that are allocated to commercial and institutional (C\&I) buildings is used for educational facilities. University campuses in Ontario recorded the second highest energy intensity consumption of $1.78 \mathrm{GJ} / \mathrm{m}^{2}$ (or $\sim 500$ $\mathrm{kWh} / \mathrm{m}^{2}$ ) compared to other provinces [5]. GHG emissions for Ontario was estimated to be 
170.2 megatonnes of carbon dioxide equivalent $\left(\mathrm{CO}_{2} \mathrm{e}\right)$ in 2014 , out of which 18.13 megatonnes, 2.27 megatonnes, and 0.89 megatonnes $\left(83.8 \mathrm{~kg} \mathrm{CO} 2 \mathrm{e} / \mathrm{m}^{2}\right)$ were allocated for the C\&I sector, educational facilities, and universities, respectively [5, 6]. Canada's current target is to reduce GHGs by 30 percent below 2005 levels by 2030, which means an absolute reduction of 200 megatonnes [7]. The goal of this study is to seek better accounting, tracking, and visualization methods to understand the opportunities to reduce GHG emissions and water and energy use in the built environment.

Many researchers have developed methodologies that measure campus resource consumption, production of waste, and GHG emissions to understand the interrelated variables that affect building performance through various accounting and visualization techniques $[13,14]$. Key concepts and methods of analysis that have emerged are urban metabolism, material flow analysis, life cycle analysis, and ecological footprint. These are briefly reviewed below in order to frame the current methodology.

In the 1960's, a Urban Metabolism (UM) was developed to better understand resource consumption of urban environments [118]. The main objective of this approach is to understand complex systems (e.g. social, environmental, and economic) when studying energy and material flows in cities [119]. The UM of a city is affected by many factors including buildings' age, urban form, technology, system efficiency, local climate, and vegetation [120]. The potential of UM is that it provides a rigorous tool at different scales when studying energy pathways [121, 122]. There are two quantities when describing UM: in terms of energy equivalent or a broader approach in terms of mass fluxes which expresses the city's water flows, materials, and nutrients. Kennedy et al. [123] showed that social, economic, and health are integrally related to UM (for example, the 
consumption of fuel is related to lifestyle). UM has been applied at different scales, from higher spatial resolution (i.e. neighborhoods [124]) to lower spatial resolution such as cities [125] and regions [126]. A study by Kennedy et al [127] developed a multi-layered indicator set of UM studies in megacities. The indicator set was designed to gather information on defining spatial boundaries, population, and economy, as well as biophysical characteristics and metabolic flows of megacities. There are some constraints and limitations on using UM including the challenges of applying it at smaller scale (i.e. campus and building levels) when data is not available at that resolution [128]. Moreover, UM focuses more on the biophysical environment with less emphasis on socio-economic issues. In addition, the assumptions made for food consumption calculations should take into account cultural differences [121].

Another approach that helps decision makers to understand the metabolism on the regional level is Material Flow Analysis (MFA). The MFA approach focuses on tracking the extraction, processing, use, and disposal or recycling of specific materials. MFA links processes and activities (i.e. construction, transportation, consumption of energy, and waste disposal) systematically [129]. The main objective of the method is to analyze, evaluate, and control material flows for a given system. Hendriks et al. [130] applied MFA on city of Vienna and the Swiss lowlands and compared the results against environmental assessment methodologies (environmental impact assessment or ecological footprint). MFA was found to be a useful tool in determining resource depletion and environmental quality for effective policymaking. Some limitations are associated with this method as it erroneously equates mass to environmental loading, which is meaningful to add up two different forms of materials having different environmental damage [131]. Furthermore, 
MFA quantifies direct consumption of a system or region while ignoring the embedded upstream processes (extraction, production, transportation, and construction) and omitting the impact of downstream (deconstruction and disposal) processes that handle a city's waste [123].

Life cycle assessment (LCA) is a process whereby the material and energy flows of a system are quantified and evaluated. The main distinction of LCA is that the assessment includes upstream use, reuse, maintenance and downstream with a temporal scope of the entire life cycle [132]. A fundamental aspect of LCA is the selection the functional unit (the unit for which the data is normalized). For instance, Norman et al. [133] showed that choice of normalizing household energy by person and by floor area made a remarkable difference in the results. LCA has some limitations as it usually models "average" systems as the data for embodied energy is not widely available and is often costly due to the effort in collecting and calculating it. Moreover, the temporal and spatial analysis of an LCA study may not provide sufficient details for some of the impact categories being considered [134, 135].

Some recent studies have suggested coupling UM and LCA to help in quantification of urban sustainability. The potential of this approach is to capture embodied environmental impacts of a metabolic flow applied for the entire life cycle and to provide an advanced method with international standards [123, 136, 137].

Several universities in North America (University of Redlands, Kwantlen, Colorado College, and UTM), Australia (University of Newcastle), and UK (Holm Lacy College, University of Wales, East Anglia, and Oxford Brookes) applied ecological 
footprint analyses [138]. Most of the studies applied to the aforementioned university campuses measured energy consumption in terms of transportation to campus, building consumption, district water use, food consumption, and waste production [139]. These studies reported ecological footprint by normalizing per unit area for each building [140] and/or based on the number of occupants (i.e. staff and students) [139]. On the other side, environmental footprint analysis is a purely environmental indicator and does not address social or economic issues necessary to comprehensively measure impacts. Moreover, in some cases, aggregation can oversimplify impacts such that individual impacts are difficult to quantify [141].

Most of the developed visualization tools available use simple lines, bar charts, and columns in presenting and comparing flow of energy and GHG emissions over time [14]. On the campus side, Pulse Energy developed an Energy Dashboard tool that shows realtime building consumption of energy, natural gas, hot water, chilled water, and steam for McGill University [142] and University of British Colombia [143]. The data were based on short-term measurements (i.e. up to three months) from meters that reported frequencies of daily, weekly, and monthly data. Another example is Arizona State University, where they used simple line graphs to show campus energy and water consumption [144]. Other tools that were developed to visualize energy consumption of buildings on the campus level and commercial buildings such as Building Dashboard [145] and Energy Efficient Education Dashboard applied to Carleton University [146], Auraria Campus, Colorado [147].

The above-mentioned models or tools aimed to empower occupants on campus to become active participants in energy management in order to reduce energy consumption 
and GHG emissions. However, they are not intended to facilitate decision making by building operators. These tools enabled an annual reduction in energy consumption that reached up to 10 percent accompanied by a reduction in GHG emissions. Another visualization model was developed to estimate the building energy consumption at the block level annually $\left(\mathrm{kWh} / \mathrm{m}^{2}\right)$ in New York City, the model only counts for building function without considering construction type or the age of the building [148]. A study by Yarbrough et al. [14] developed a new visualization tool on the campus level to understand the relationship between individual building peak demand and the campus peak energy use based on data provided every 15 minutes by energy meter and billing peak. Another study in 2009 used energy dashboard to improve the visibility of energy consumption of four selected mixed-use buildings in the University of California at San Diego; however, this study did not include other individual building usages [149]. McCusker [150] used color mapping to visualize building energy consumption and GHG emissions at the University of Massachusetts Amherst. Based on the previous proposed models or tools, there are limited applications that target building operators and other stakeholders on campus such as visualizing HVAC and lighting systems which can support building operators to operate their buildings optimally with less effort [106].

An especially effective method to visualize of materials and energy flows that have existed for over 100 years is the Sankey diagram [101]. Sankey diagrams are comprised of arrows of varying widths, where the width indicates relative magnitude of flow and the direction indicates the connection between sources and sinks for each flow. Sankey diagrams allow resource flows to be visualized within complex systems with interacting subsystems. They are particularly useful for understanding relative flows of resources and 
identifying opportunities for which waste outputs could be recirculated as inputs back into the system.

Previous notable applications of Sankey diagrams have been applied to chemical plants, power plants, and nationwide energy production and distribution systems. However, there are limited applications on the building and campus scales [151]. Sankey diagrams can be applied both for visualizing performance of existing systems as well as during design using modelling and simulation. A recent study by Singer \& Simon [152] used Sankey diagrams to visually represent energy usage among commercial buildings within the Navy Yard. However, the created Sankey diagrams focused only on the building level using Energy Information Administration (EIA) Commercial end-use data without using meter readings. Another study by Sims et al. [153] used Sankey diagrams to illustrate the primary energy consumption by the main sectors (i.e. transport, industry, and buildings) including the losses due to inefficiceny and ditribution losses. However, this study did not illustrate the performance of different building types, ages, and usages. A limited number of building design and analysis tools, such as CASAnova software [154] and Sefaira [155] use Sankey diagrams for visualizing predicted energy use. A study by Fedoruk et al. [156] applied Sankey diagram to compare between the designed and the actual performance on one of the buildings at the University of British Columbia to investigate the performance failures during operation.

The main objective of this research is to develop a methodology to analyze and visualize energy, water, natural gas, utility cost, and GHG emissions data at the building and campus scale using Sankey diagrams and other graphical methods, also estimating data on per unit area and per occupant bases. The study utilizes spatial-temporal analysis of 
different building vintages and functions to facilitate the decision-making by building operators, campus planners, and other stakeholders. Once the methodology was developed, it was applied to a case study: Carleton University campus in Ottawa, Canada. This study was followed by a survey targeting building operators to investigate the usefulness and applicability of the developed methodology in their decision-making process. Finally, challenges and future research needs are discussed.

\subsection{Methodology}

The main objective of this research was to develop a methodology to obtain, process, analyze, and visualize resource consumption and GHG emissions data at the building and campus scale. The methodology focused on energy, natural gas, and water consumption by the main power plant and by different buildings on campus accompanied by GHG emissions as shown in Figure 3.1. Moreover, the study accounts for converting primary to secondary energy including generation, transmission, and distribution processes. For the natural gas delivered to the campus, the fuel processing and transportation were taken into account. While, for district water, the amount of energy required for treatment and pumping from the grid was also considered in the study. Notable exclusions from this study include food, office and educational supplies and goods, and transportation. In short, only resources that are metered and their upstream impacts are assessed.

This section describes a methodology for quantifying and normalizing resource use and GHG emissions for typical campus configurations. 


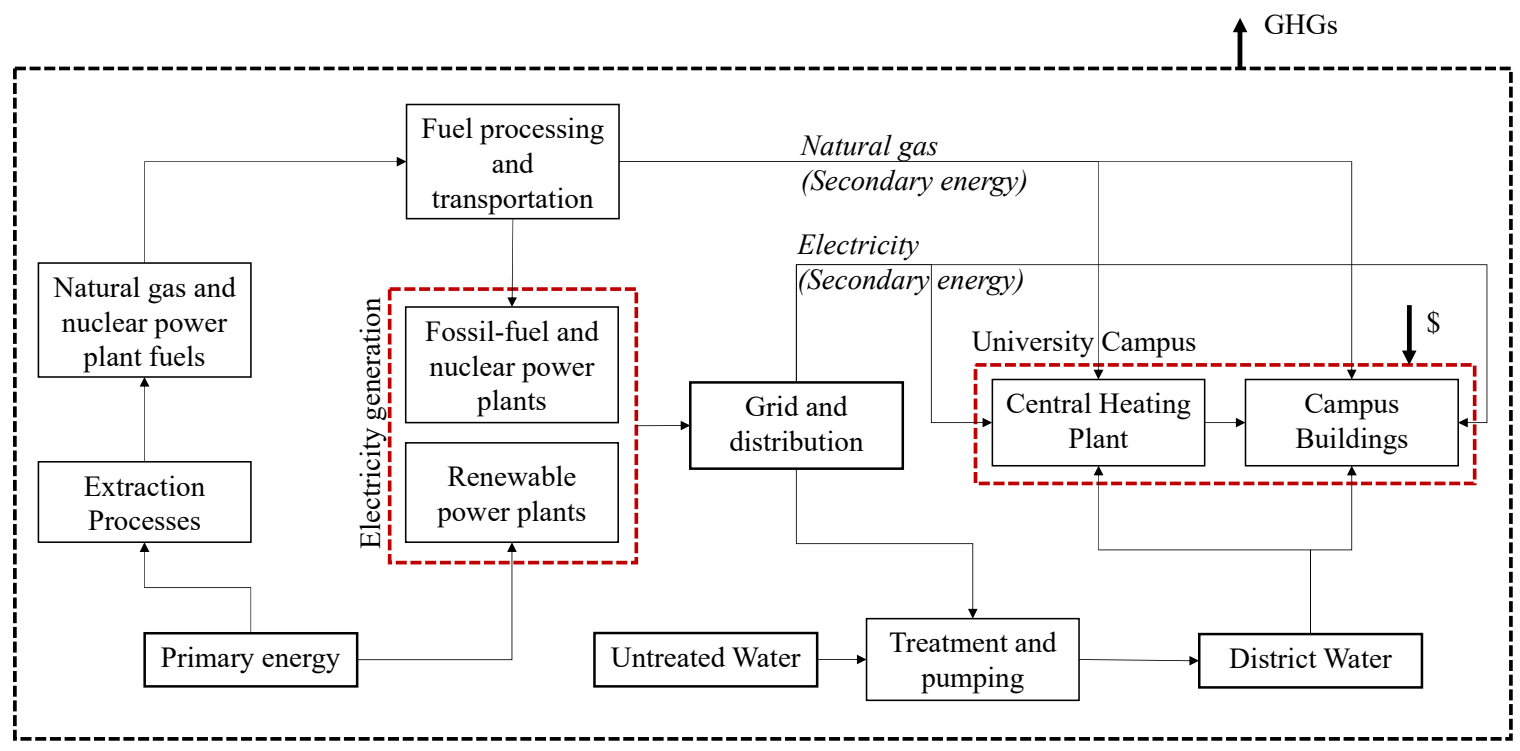

Figure 3.1: Flowchart showing the main scope of the study

\subsubsection{Central Heating Plant (CHP)}

Many communities and campuses have centralized heating plants in order to reduce equipment size and cost, improve efficiency, and centralize operations. These are likely fed by natural gas or coal and may produce electrical power and thermal energy (i.e., cogeneration), or merely thermal energy. The current discussion is in the context of the Carleton case study. The steam is generated at the central heating plant (CHP) by burning large quantities of natural gas, while a small amount of electricity is used to run the steam boilers. Saturated steam is generated at high pressure (550 $\mathrm{kPa}$ or more) and distributed to each building through insulated pipes. The delivered steam to the buildings passes first through a heat exchanger that uses the thermal energy to provide heat to heating coils, radiators, radiant panels, and hot water. Some of the steam is returned back in the form of condensate water to the CHP, while some thermal energy is lost to the environment surrounding the distribution network of pipes. The amount of the water loss through leaking pipes is replaced by the district water supply. Figure 3.2 illustrates energy and 
water consumption by CHP and different forms of heat losses.

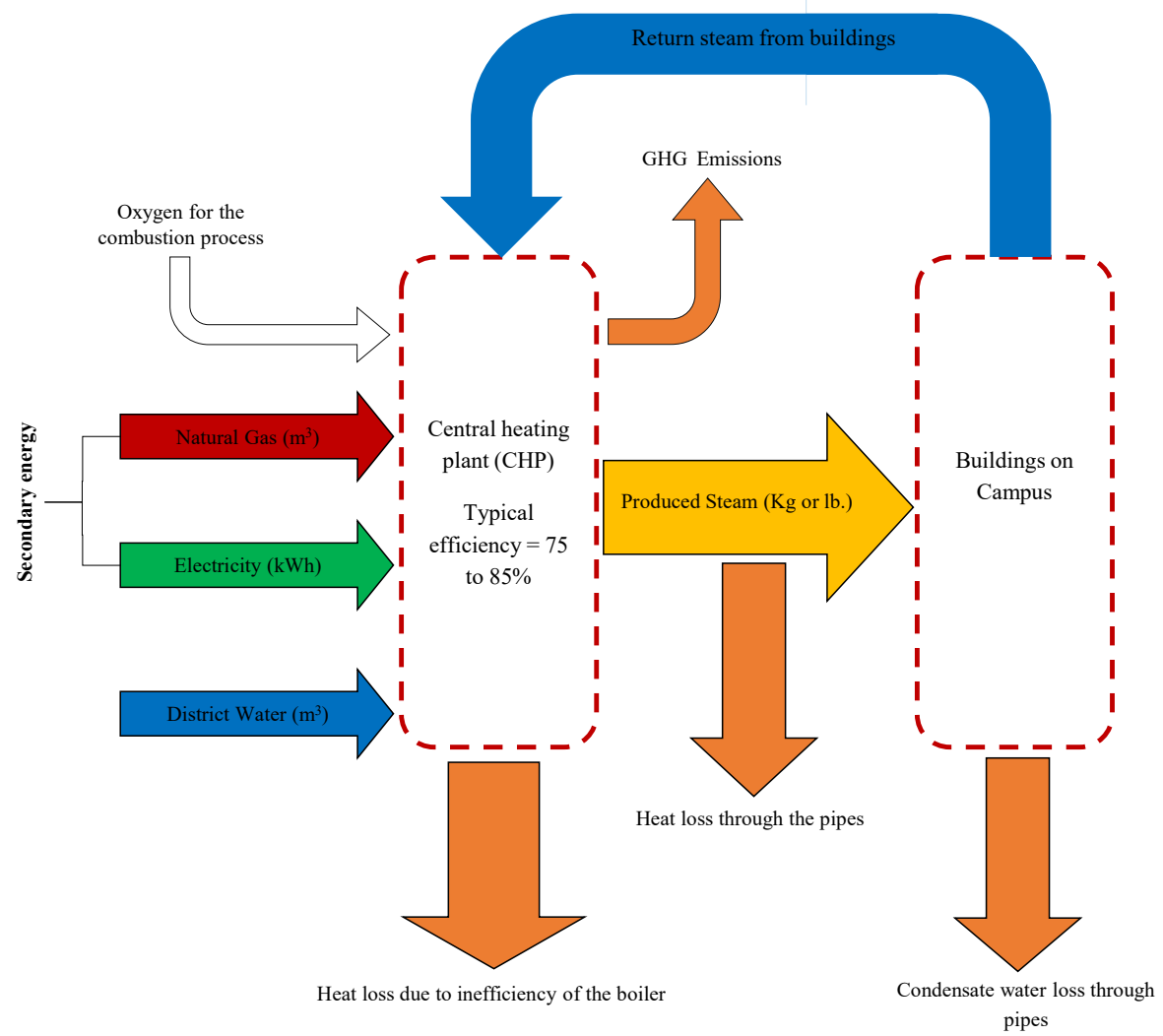

Figure 3.2: Energy and water consumption by CHP and different forms of heat losses

Some of the energy losses by the boiler are exhausted to the environment and some are radiated to the surroundings based on the efficiency of the boiler. The other source of heat loss is due to the distribution losses through the pipes. Distribution losses were calculated by recording the steam meter readings for steam mass production by boilers and the sum of steam mass consumption by each building over specific time as shown in Eq. (3.1):

Distribution loss through pipes

Equation (3.2) describes the efficiency of the boiler, which is defined as the ratio 
of steam produced by the boilers to the total energy embodied in the steam production including electricity and natural gas. Since the natural gas input is much higher than the electricity input, they were directly combined in the current study for quoting efficiency. However, their mutual primary energy and GHG implications should be considered where electrical energy input is significant.

$$
\text { Efficiency }(\eta)=\frac{\text { Steam energy produced }}{\text { Electricity and natural gas involved in steam production }} \times(100 \%)
$$

The steam mass production was obtained from the steam meters installed at the exit of the boiler. While, the electricity and natural gas consumption were obtained from meters installed at the boiler's entry. Thus, the first step is to convert the steam and natural gas units to a common energy unit. The average typical value for the CHP efficiency is from 75 to 85 percent [157].

\subsubsection{Accounting for Building Size and Energy Use Intensity}

In order to compare energy consumption of each building, energy usage intensity (EUI) should be considered. The gross building floor area were obtained from the documented drawings for each building provided by the facility management on campus. The amount of energy required for non-building infrastructure such as exterior lights were considered small compared to the rest of building energy consumption. The meters typically allocated electricity use for exterior lighting to the nearest building. The amount of energy used by buildings is called secondary or site energy. The secondary energy was calculated based on the raw data reported by each meter. However, site EUI do not count for energy required to generate and transmit the specific energy type. 


\subsubsection{Primary to Secondary Conversion Factors}

Primary or source energy is another metric that should be considered. The primary energy is the total energy at source required to supply energy to campus, including all energy required for extraction, processing, transportation, and transmission of energy as well as conversion losses. The primary energy source is also useful in energy planning when developing long-range policies and for energy analysts who are concerned with broader energy or environmental issues (such as energy efficiency measures and carbon emissions from energy sources) [158]. On the electricity side, it includes power plant efficiency and losses due to transmission and distribution (T\&D). The primary and secondary sources of energy are assumed to be equal to one for electricity production from hydroelectric power, solar energy, and wind, as the electricity produced is derived from renewable energy sources. However, some of the energy is lost due to transmission and distribution of energy to facility $[159,160]$. In Ontario, the transmission and distribution losses are around 6.5 percent, out of which 2 percent is allocated for transmission losses [161]. The primary to secondary source ratio for grid electricity and natural gas were calculated as shown in Eq. (3.3) and Eq. (3.4), respectively [162]:

$$
\begin{gathered}
\text { Primary to Secondary source ratio }=\frac{\text { Primary energy inputs }}{\text { Electrical energy output }} \\
\text { Primary to Secondary source ratio }=\frac{\text { Natural gas input }}{\text { Processed natural gas delivered }}
\end{gathered}
$$

For power plants that use fossil fuels to generate electricity, including transmission and distribution losses, the above equation could be expressed as Eq. (3.5): 


$$
\text { Primary energy }=\left(\frac{1}{\text { Plant efficiency }}\right) \times\left(\frac{1}{1-T \& D \text { losses }}\right) \times \text { Secondary energy }
$$

For renewable energy sources, such as wind, solar, and hydro, transmission and distribution losses - not plant efficiency - were taken into consideration as shown in Eq. (3.6):

$$
\text { Primary energy }=\left(\frac{1}{1-T \& D \text { losses }}\right) \times \text { Secondary energy }
$$

According to the Independent Electricity System Operator (IESO), electricity generated in Ontario in 2013-2014 was comprised of approximately 62 percent nuclear, 24 percent hydro, 10 percent natural gas, 4 percent wind power, 0.19 percent Bio-fuel, and 0.012 percent solar [163]. Note that the breakdown of electricity sources varies seasonally, but that the current study is focused on the annual scale. Hydro and nuclear power plants represent base generation, while natural gas power plants are used to cover peak loads [163]. If the temporal resolution of this study were increased, the temporal resolution of the power generation sources would have to be incorporated. For instance, the temporal resolution of the electricity data of the current case study is not adequate to determine if the timing of Carleton's electricity demand results in a different breakdown of electricity supply sources than Ontario's annual average.

The efficiency of nuclear, natural gas, and biomass power plants were determined by the total amount of fuel consumption for electricity generation and the amount of electricity generated from each fuel type provided by statistics Canada $[164,165]$. The efficiency of nuclear, natural gas, and biomass power plants were found to be 34,45 , and 40 percent, respectively. For the delivered natural gas to the campus, the fuel processing and transportation were considered. The secondary to primary energy conversion factors, 
as calculated using the method above, are shown in Table 3.1. These values represent typical Canadian power plant efficiencies and are not specific to Ontario's infrastructure.

Table 3.1: Secondary to primary conversion factors

\begin{tabular}{|c|c|c|c|}
\hline \multicolumn{2}{|c|}{ Energy Type } & Primary to Secondary & Source \\
\hline \multicolumn{2}{|c|}{ Natural Gas } & 1.047 & [166] \\
\hline \multirow{6}{*}{$\begin{array}{l}\text { Electricity } \\
\text { generation }\end{array}$} & Nuclear & 3.145 & \multirow{3}{*}{$\begin{array}{l}\text { Based on the efficiency of the plants } \\
\text { including T\&D losses }[167,168]\end{array}$} \\
\hline & Bio-fuel & 2.67 & \\
\hline & Natural Gas & 2.37 & \\
\hline & Hydro & 1.069 & \multirow{3}{*}{$\begin{array}{c}\text { Based on T\&D losses in Ontario } \\
{[161]}\end{array}$} \\
\hline & Solar & 1.069 & \\
\hline & Wind & 1.069 & \\
\hline
\end{tabular}

Moreover, the amount of electrical energy embedded of water and wastewater was also considered in the study. The average energy intensity for pumping and treatment of municipal water and wastewater services in Ontario are 0.65 and $0.52 \mathrm{kWh} / \mathrm{m}^{3}$, respectively [169]. The municipal water and wastewater losses were estimated based on aggregate data for the institutional and commercial building sector in Ontario based on the study by Maas [170]. It was found that the municipal water loss from the plant to campus is approximately 12 percent. A net of eight percent of the delivered municipal water is lost due to pipe leakage, evaporation, and irrigation. Furthermore, the wastewater loss prior leaving the campus is approximately five percent. Equations (3.7) and (3.8) were used to determine the total embedded electrical energy for campus water and wastewater, respectively.

$$
\begin{gathered}
E_{\text {emb,elect (water) }}=\left[1.12 \times Q_{\text {cam, water }}\right] \times 0.65 \mathrm{kWh} / \mathrm{m}^{3} \\
E_{\text {emb,elect (wastewater) }}=\left[0.92 \times(1-0.05) Q_{\text {cam, water }}\right] \times 0.52 \mathrm{kWh} / \mathrm{m}^{3}
\end{gathered}
$$

Where, $E$ emb, elect, (water) is the total electrical energy embedded for pumping and 


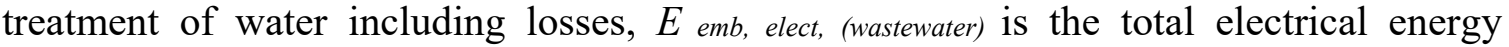
embedded for pumping and treatment of wastewater including losses, and $Q_{\text {cam, water }}$ is the total campus water consumption, obtained from water meters.

\subsubsection{Accounting for Occupancy}

Another form of building performance assessment is to estimate the normalized resource consumption and GHG emissions per occupant. Occupants are the major contributors to building energy use. The occupancy patterns can vary widely depending on the space type and usage. The current objective was to estimate the allocation of total campus occupants by building for accounting purposes. However, it is acknowledged that these are often estimates based on engineering judgment rather than from measurement.

For office spaces, the allocated office spaces for each staff/employee were obtained from the university database. While, the number of occupants in residences was calculated as shown in Eq. (3.9):

$\begin{aligned} & \text { Number of occupants } \\ & \text { per residence building }\end{aligned}=\frac{\begin{array}{c}\text { Number of bedrooms } \\ \text { in a specific building }\end{array}}{\text { Total number of bedrooms in residences }} \times \begin{gathered}\text { Total number of } \\ \text { full-time equivalent } \\ \text { occupants in residences }\end{gathered}$

The total number of students on campus was distributed based on the capacity (i.e. number of chairs) of each building. The number of students in each classroom/lecture hall/lab per building was estimated using Eq. (3.10):

$$
\begin{gathered}
\text { Number of students } \\
\text { per building }
\end{gathered}=\frac{\text { Capacity of specific building }}{\text { Total capacity of student facilities on campus }} \times \begin{gathered}
\text { Total number of } \\
\text { full-time } \\
\text { equivalent students }
\end{gathered}
$$


For the library, arena, gym, and other sports facilities, the number of occupants per each facility was based on the load capacity determined by the Ontario Building Code [171]. Further infrastructure and investigations would be required to track the actual number of occupants per building. For example, security cameras, computer laboratory log-in events, or classroom $\mathrm{CO}_{2}$ concentration could be used to estimate occupancy.

\subsubsection{Accounting for Building Age and Usage}

It was expected that building age would affect energy consumption due to ageing envelopes and equipment and lower building standards in the past, thus this relationship was explored [172]. However, in a typical campus, such as Carleton University, there is a large variety of building functions. These functions include athletics, ancillary (i.e. a building used for storage, machinery, dwellings, or offices near a main structure), academic, administrative, residence, research, and parking. Moreover, some buildings have multiple activities (i.e. mixed use), such as buildings that consists of administrative offices, food courts, and classrooms. For such buildings, it is difficult to estimate energy consumption due to a wide variety of uses and energy services [173]. Thus, more investigation regarding energy demand of individual buildings is required.

\subsubsection{Calculations for Equivalent Carbon Dioxide Emissions (CO2e)}

Two major sources of greenhouse gas emissions on a campus include those directly generated on the campus footprint (e.g., from natural gas combustion) and those which are emitted upstream of campus in order to provide resources to campus (e.g., natural gas-fired power plants to supply electricity and pump water). For Carleton University, the main source of greenhouse gas (GHG) emissions is the central heating plant, where the boilers burn large quantities of natural gas to heat most campus buildings. 
Each greenhouse gas has a different global warming potential (GWP) and persists for a different length of time in the atmosphere [174]. The three main GHGs are carbon dioxide $\left(\mathrm{CO}_{2}\right)$, methane $\left(\mathrm{CH}_{4}\right)$, and nitrous oxide $\left(\mathrm{N}_{2} \mathrm{O}\right)$, along with water vapor. Equivalent carbon dioxide $\left(\mathrm{CO}_{2} \mathrm{e}\right)$ is the common unit to quantify the $\mathrm{GHG}$ emissions in terms of the equivalent impact of $\mathrm{CO}_{2}$ alone. The equivalent carbon dioxide values for $\mathrm{CO}_{2}$, $\mathrm{CH}_{4}$, and $\mathrm{N}_{2} \mathrm{O}$ are 1,25 , and 298, respectively [153]. The amount of $\mathrm{CO}_{2} \mathrm{e}$ released by combusting different fuel types can be quantified as Eq. (3.11) [175]:

$$
\text { Total } G H G \text { emissions }=\Sigma\left(\begin{array}{c}
\text { energy consumption } \\
\text { by fuel type }
\end{array}\right) \times\left(\begin{array}{c}
G H G \text { emission factor } \\
\text { by fuel type }
\end{array}\right)
$$

The electricity emission factor depends on the generation mix of the electricity grid. The $\mathrm{CO}_{2} \mathrm{e}$ intensity factor in Ontario dropped from $0.3 \mathrm{~kg} \mathrm{CO}_{2} \mathrm{e} / \mathrm{kWh}$ in 2003 to 0.085 and $0.05 \mathrm{~kg} \mathrm{CO} 2 \mathrm{e} / \mathrm{kWh}$ in 2013 and 2014, respectively, due to the reduction and eventual elimination of coal-fired power plants [176]. The average net emission factors in Ontario for electricity, natural gas, and bio-fuel are listed in Table 3.2.

Table 3.2: $\mathrm{CO}_{2}$ e Emission Factors

\begin{tabular}{|l|c|c|c|}
\hline Energy Type & \multicolumn{2}{|c|}{$\mathrm{CO}_{2} \mathbf{e}$ Emission factors } & Source \\
\hline Electricity & $0.014 \mathrm{~kg} \mathrm{CO} \mathrm{eJ}_{2} / \mathrm{MJ}_{\text {electrical }}$ & $0.05 \mathrm{~kg} \mathrm{CO} \mathrm{e}_{2} / \mathrm{kWh}_{\text {electrical }}$ & {$[176]$} \\
\hline Natural Gas & $0.056 \mathrm{~kg} \mathrm{CO} \mathrm{eJ}_{2} / \mathrm{MJ}_{\text {thermal }}$ & $0.201 \mathrm{~kg} \mathrm{CO}_{2} \mathrm{e} / \mathrm{kWh}_{\text {thermal }}$ & {$[177]$} \\
\hline Bio-fuel & $0.0083 \mathrm{~kg} \mathrm{CO} \mathrm{KO}_{2} \mathrm{e} / \mathrm{MJ}_{\text {thermal }}$ & $0.03 \mathrm{~kg} \mathrm{CO} \mathrm{CO}_{2} \mathrm{e} / \mathrm{kWh}_{\text {thermal }}$ & {$[178]$} \\
\hline
\end{tabular}

\subsection{Application: Carleton University Campus}

Carleton University is located in Ottawa, Canada $\left(45.32^{\circ} \mathrm{N}\right.$ and $\left.75.67^{\circ} \mathrm{W}\right)$ spreading over an area of 54.63 hectares. Ottawa's climate is a humid continental climate, characterized by hot-humid summers with an average temperature of $21^{\circ} \mathrm{C}$ and cold-humid winter days with an average of $-10.3^{\circ} \mathrm{C}[179]$. 
In 2013, Carleton University started DCI (Digital Campus Innovation) project. The main objective of the project is to provide a useful platform for Facilities Management and Planning (FMP) department on campus and other stakeholders that can be expanded as a generalized methodology for other campuses and communities. This can be done by applying an integrated BIM (Building information modeling), BPS (building performance simulation), and continuous monitoring and commissioning [32]. One of the crucial steps is to obtain, process, analyze, and visualize data for energy, water, and natural gas consumption and GHG emissions on the campus level, which is the main scope of this study.

Carleton University consists of 45 buildings and a main central heating plant (CHP) as shown in Figure 3.3. The annual utility cost is of $\$ 12$ million, or an average of $\$ 270,000$ per building and about $\$ 400$ per student or employee. For heating purposes, most of the buildings are provided with steam produced from the CHP, while other buildings have their own boilers that consume natural gas.

A tunnel connects the buildings on campus, which is heated by steam supplied by the Athletic building (bldg. \#9). Thus, a high value of steam consumption was recorded for this building. There is no mechanical ventilation system applied to the tunnel. Air is circulated through the tunnel due to stack effect. All buildings on campus and the CHP are supplied by district water except those for parking purposes. 


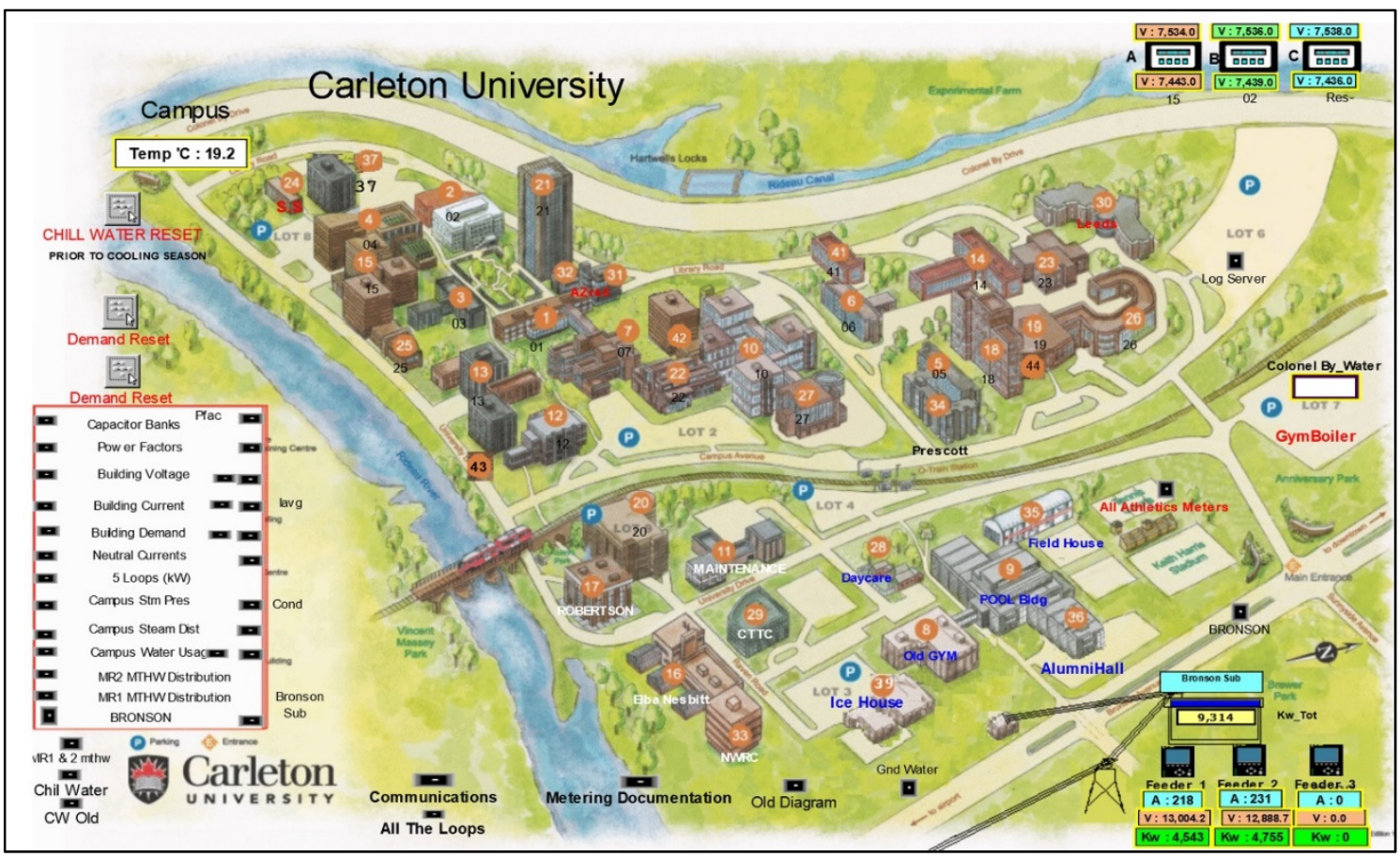

Figure 3.3: Carleton Campus layout

\subsubsection{Data Acquisition}

Meters installed in each building and in the CHP recorded electricity, steam, natural gas, and water consumption. Raw data were reported on a server operated by the Facilities Management and Planning (FMP). With this service, annually, monthly, and hourly data for the current year were obtained. However, some modest errors are associated with meters as some give negative values or cumulative readings. A report published in 2014 by Honeywell mentioned that the water consumption patterns were skewed by a series of meter reading errors from March to December 2012 [180]. Thus, an average data for water consumption from 2009 to 2011 were recorded for those meters. The remaining data were consistent with the utility billing data at the campus scale. Information about building names, activities, ages, and floor areas are reported in Table 3.3.

Steam produced by the CHP is the main source of energy for heating purposes. 37 
buildings rely on steam produced by the CHP, while seven buildings consume natural gas directly for their own boilers for heating purposes. For the parking garage, there is no supply of heating or cooling. The CHP consumes natural gas and a small amount of electricity to run the boilers. Moreover, the plant is supplied by district water, while approximately 90 percent of the steam is circulated back to the plant and the rest are lost through the pipes. There are 22 chillers on campus providing cooling to buildings. Some buildings, such as Mackenzie (bldg. \#10), Minto (bldg. \#27), and Canal (bldg. \#42) share the same chiller.

Table 3.3: Building name corresponding to building number

\begin{tabular}{llcll}
\hline Building \# & Building Name & Built-Up Year & Building type \\
\hline BLDG11 & Maintenance & 1960 & Ancillary \\
BLDG19 & Commons & 1960 & Ancillary \\
BLDG28 & Colonel by Childcare Centre & 1990 & Ancillary \\
BLDG29 & CTTC & 1990 & Ancillary \\
\hline BLDG33 & NWRC (Natural Wildlife & 2000 & Ancillary / Academic \\
& Research) & 1960 & Academic \\
\hline BLDG2 & MacOdrum Library & 1960 & Academic \\
BLDG3 & Paterson Hall & 1960 & Academic \\
BLDG4 & Southam Hall & 1960 & Academic \\
BLDG10 & Mackenzie & 1960 & Academic \\
BLDG15 & Loeb & 1960 & Academic \\
BLDG16 & Nesbitt & 1960 & Academic \\
BLDG21 & Dunton & 1970 & Academic \\
BLDG22 & Architecture & 1970 & Academic \\
BLDG23 & St.Patrick's & 1990 & Academic \\
BLDG27 & Minto Case & 2000 & Academic \\
BLDG31 & Azrieli Theatre & 2000 & Academic \\
BLDG32 & Azrieli Classroom Pavilion & 2011 & Academic \\
BLDG42 & Canal & 2011 & Academic \\
BLDG43 & River Building & 2005 & Academic \\
BLDG37/38 & HCI VSIM & 1960 & Administrative \\
\hline BLDG17 & Robertson & 1960 & Academic / Admin \\
\hline BLDG1 & Tory & 1960 & Ancillary / Academic/ Admin \\
\hline BLDG7 & Unicentre & 1960 & Research \\
\hline BLDG12 & Steacie & 1960 & Research \\
BLDG13 & Herzberg & &
\end{tabular}




\begin{tabular}{llll} 
BLDG24 & Social Science & 1970 & Research \\
BLDG25 & Life Science & 1970 & Research \\
\hline BLDG8 & Gym & 1960 & Athletics \\
BLDG9 & Athletics & 1960 & Athletics \\
BLDG35 & Field House & 2000 & Athletics \\
BLDG36 & Alumni Hall & 2005 & Athletics \\
BLDG39 & Ice House & 2005 & Athletics \\
BLDG40 & Tennis Bubble & 2005 & Athletics \\
\hline BLDG5 & Renfrew & 1960 & Residence \\
BLDG6 & Lanark & 1960 & Residence \\
BLDG14 & Russell / Grenville & 1960 & Residence \\
BLDG18 & Glengary & 1960 & Residence \\
BLDG26 & Stormont / Dundas & 1990 & Residence \\
BLDG30 & Leeds & 2000 & Residence \\
BLDG34 & Prescott & 2000 & Residence \\
BLDG41 & Frontenac & 2005 & Residence \\
BLDG44 & Lennox \& Addington House & 2011 & Residence \\
\hline BLDG20 & Parking Garage & 1960 & Parking \\
\hline
\end{tabular}

\subsubsection{Building Age and Functions}

The 45 buildings on campus were built from 1960 until 2011 and include a large number of facilities and building functions. These functions include all aforementioned functions. Moreover, some buildings are mixed-use, such as the University Center, Robertson Hall, and National Wildlife Research buildings.

\subsubsection{Number of Occupants}

The total number of full-time equivalent students is 25,390 , out of which 22,029 and 3,361 are undergraduate and graduate students, respectively. Moreover, the number of faculty members, staff members, contract instructors, library staff, and teaching assistants (all of whom are graduate students) on campus are 841, 1023, 679, 108, and 1748, respectively. The number of employees and their allocated office spaces were obtained from the university employee directory. Based on Carleton University statistics in 2015, there are approximately 3,600 beds in residence, which are essentially fully occupied, as 
the capacity of the residences is equal to the number of students living in residences [181]. While the number of students allocated per each classrooms, lectures, or labs were estimated using the method described in the methodology section. It was found that the number of students is more than classroom seats; but this is expected since students typically have 15-25 hours of class per week.

Based on the Ontario Building Code, the load capacity for library, restaurants, gymnasium, and swimming pool are $3-9 / \mathrm{m}^{2}, 1.5 / \mathrm{m}^{2}, 1.5 / \mathrm{m}^{2}$, and $4 / \mathrm{m}^{2}$, respectively. For sport facilities, the number of players was estimated based on the number of members per team [182]. The average number of spectators per arena was estimated based on the number of spectators per event and the total number of annual events, which was obtained from the university database as shown in Eq. (3.12).

$$
\text { Average number of spectators }=\frac{\text { Number of spectators per event } \times \text { Number of events }}{365 \text { days }}
$$

Figure 3.4 shows the number of occupants and the gross area of each building. The highest occupancy levels were calculated for Southam Hall due to the large number of facilities (i.e. classrooms, theater, lecture halls, and television studios). Recall that the values do not represent instantaneous occupancy but are rather estimated in order to normalize campus resource use and GHG emissions on a per person basis. 


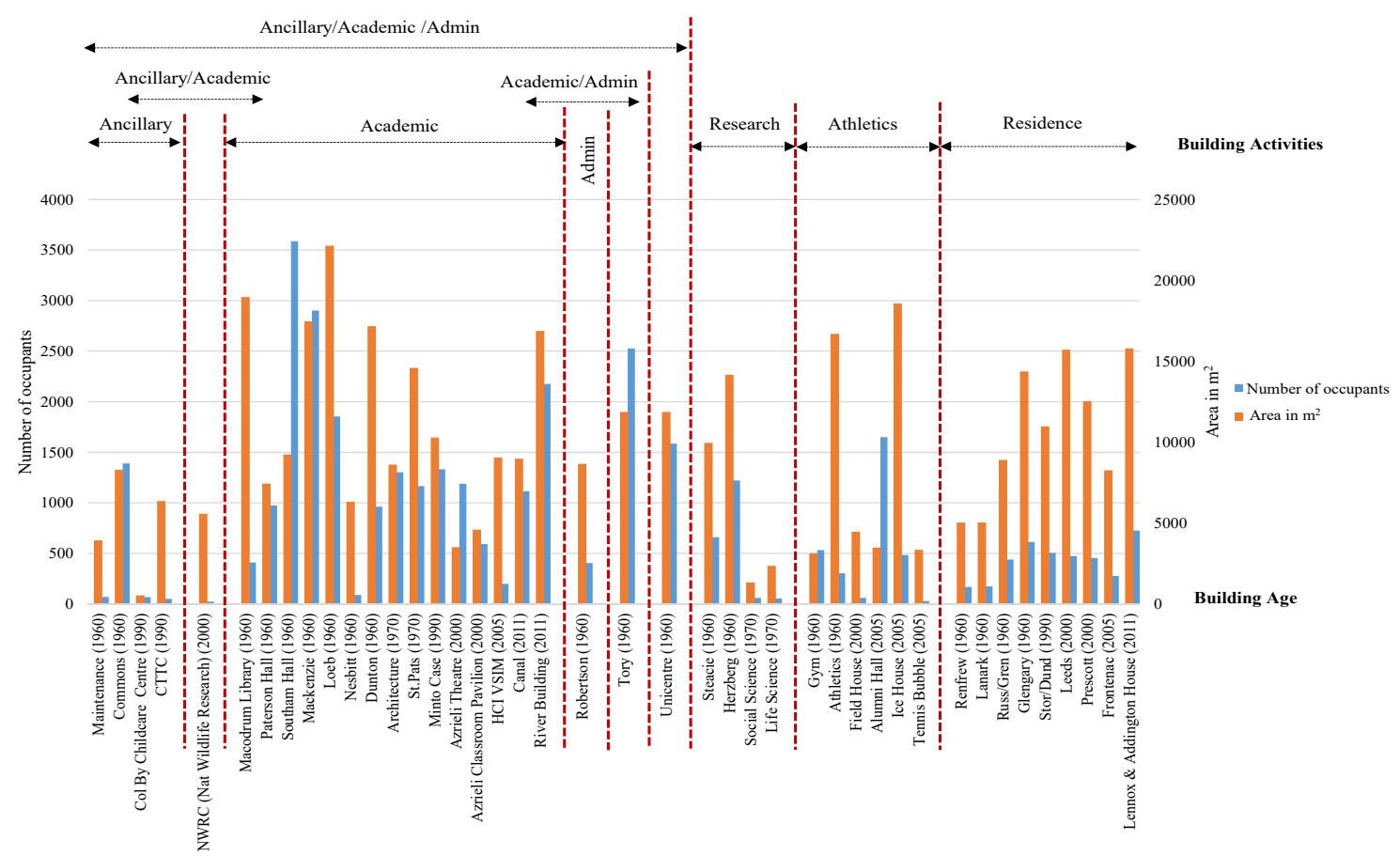

Figure 3.4: Number of occupants and area for each building

\subsection{Data Visualization}

In this section, samples of data visualization using Sankey diagram are presented. These diagrams include visualization of electricity, natural gas, water, and steam consumption, GHG emissions, and utility costs on annual basis. Sankey diagrams were rendered in a browser using Scalable Vector Graphics (SVG). The layout was derived from D3's Sankey layout code provided by Google Developers that provided more flexibility in organizing nodes, colors, and font sizes [183]. In order to create nodes with the connection links, a set of rows containing data for source, target, and magnitude for each flow was created using Microsoft excel files (CSV format). For more legibility of the diagrams, building numbers were used instead of using building names.

\subsubsection{Energy Consumption}

The first step was to show the consumption of primary energy sources (nuclear, natural gas, wind, hydro, solar, and bio-fuel). Some of this energy is converted into useful 
energy, while some is lost due to generation, distribution, and transmission processes. For the CHP, some of the supplied energy is converted into useful energy (i.e. steam production). Some heat losses were recorded due to the inefficiency of the plant and the distribution losses through the pipes from the plant to the buildings. All forms of energy (i.e. electricity, steam, and natural gas) in this diagram were converted to one common energy unit (GWh) as shown in Figure 3.5.

\subsubsection{Mass flow (District Water, Steam, and Natural Gas)}

The aim of this diagram is to show mass flow of district water, steam, and natural gas and their distribution by different sources. Appendix A. 1 shows mass production of steam by the CHP and its distribution to buildings including return steam from buildings. Moreover, steam losses due to inefficiency and distribution were also taken into consideration. The common unit used to represent mass flow is in tonnes.

\subsubsection{Greenhouse gas emissions $\left(\mathrm{CO}_{2} \mathrm{e}\right)$}

As pointed out earlier, factors contributing in GHG emissions are from the generation and transmission of electricity, natural gas combustion from the CHP, and natural gas consumed by some buildings for heating or for food preparation purposes. The common unit used to display $\mathrm{CO}_{2}$ e emissions is tonnes as shown in Appendix A. 2.

\subsubsection{Utility Cost}

The total energy and water expenditures of the utilities at the campus and building scales are shown in Appendix A. 3. The utility cost is allocated to the grid electricity, natural gas, and district water. Moreover, the heat losses from the CHP due to inefficiency of the CHP and distribution losses through pipes are also included. 


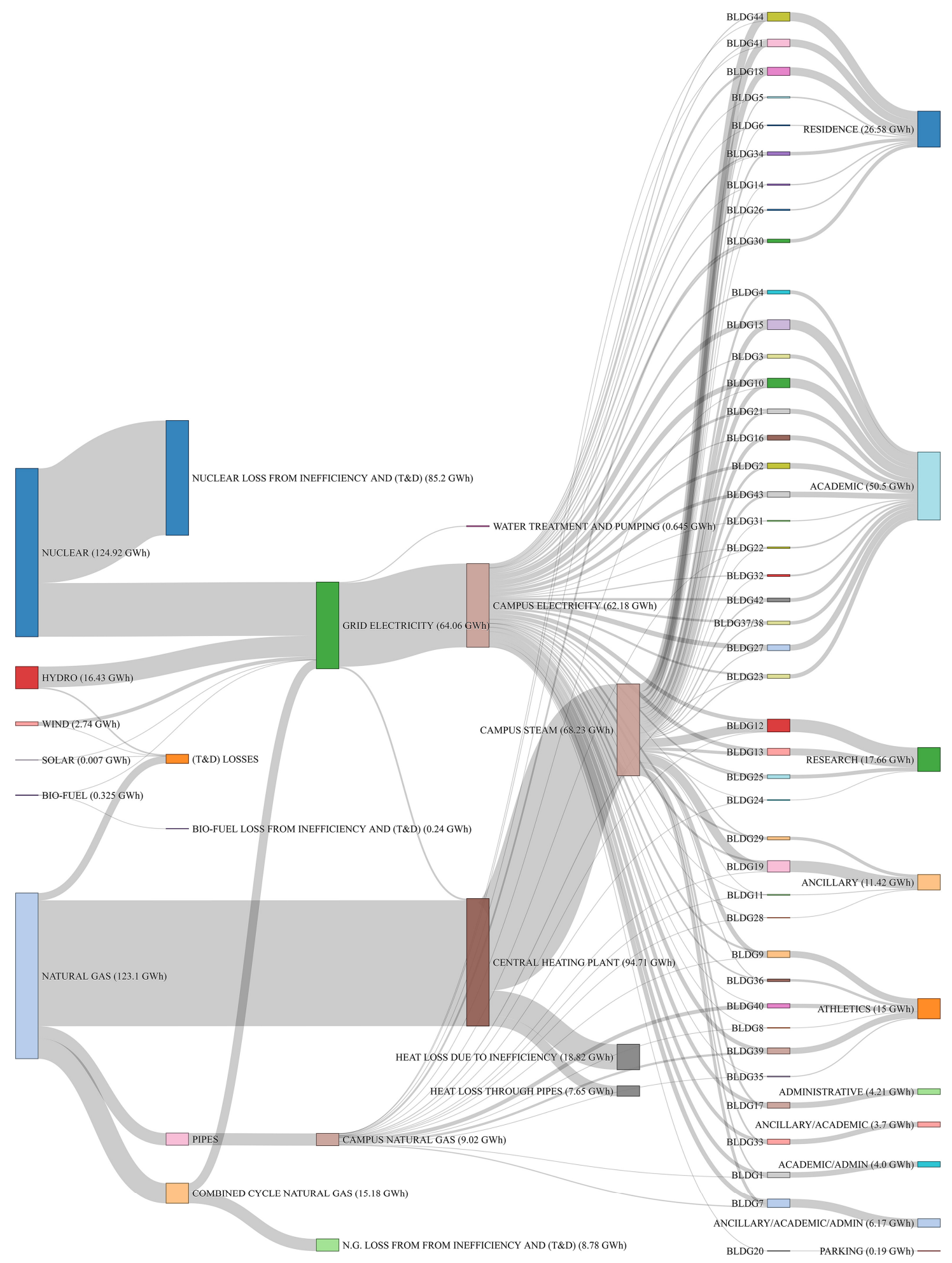

Figure 3.5: Sankey diagram showing annual energy consumption 


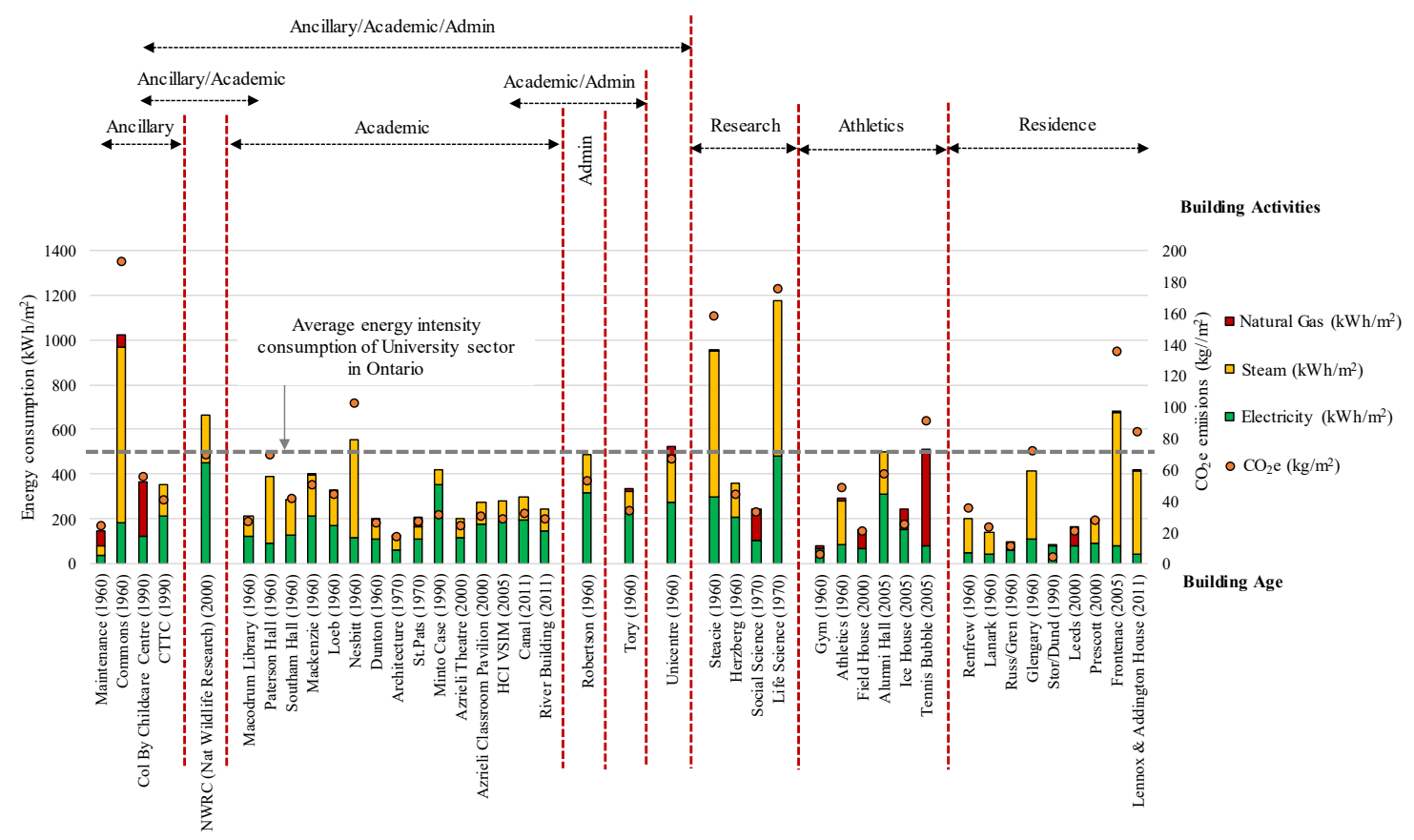

Figure 3.6: Normalized annual energy consumption and $\mathrm{CO}_{2} \mathrm{e}$ emissions per unit area

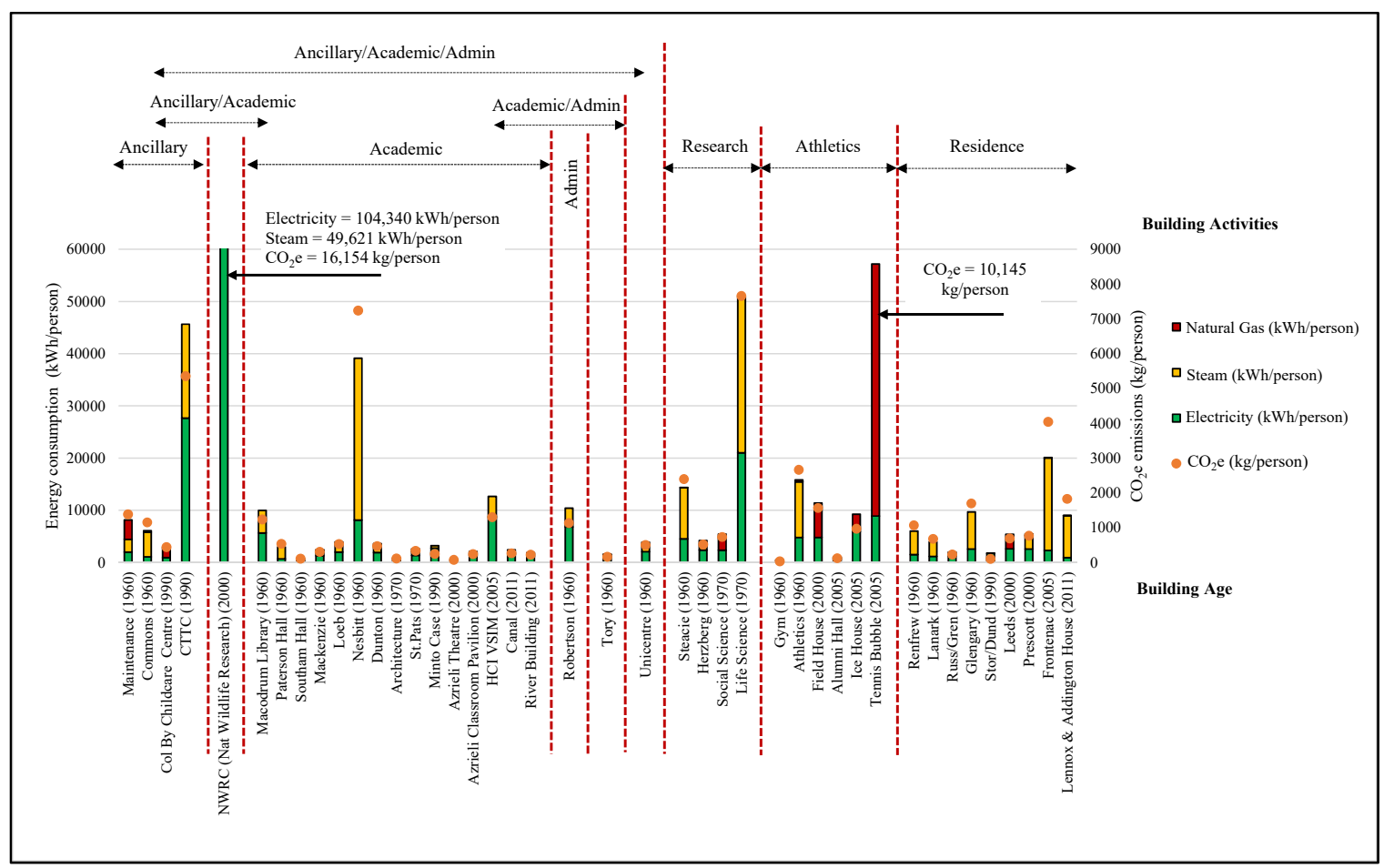

Figure 3.7: Normalized annual energy consumption and $\mathrm{CO}_{2} \mathrm{e}$ emissions per person 


\subsection{Results and Discussion}

This section consists of two parts; the first part focuses on the results from Sankey diagrams on the campus total area taking into account the normalized data per unit area and occupants. The second part includes results from the survey applied to building operators on Carleton University campus on evaluating Sankey diagrams for tracking building/campus energy and water use.

As buildings on campus have different functions and ages, it was useful to represent energy, water, and steam consumption, GHG emissions, and utility cost for the campus total area and normalized data per unit area $\left(\mathrm{m}^{2}\right)$ and per occupant in order to determine the performance of each building.

The average energy consumption in $\mathrm{GWh}$ and $\mathrm{CO}_{2} \mathrm{e}$ emissions in tonnes are shown in Figure 3.5 and Appendix A. 2, respectively. The central heating plant (CHP) recorded the highest energy consumption with a total of $94.71 \mathrm{GWh}$, out of which $68.23 \mathrm{GWh}$ was converted to steam and supplied to the buildings, while $18.82 \mathrm{GWh}$ and $7.65 \mathrm{GWh}$ were recorded for heat loss due to inefficiency of the plant and the distribution losses through the pipes, respectively. The CHP emits the highest $\mathrm{CO}_{2} \mathrm{e}$ at $18.75 \times 10^{3}$ tonnes. On the other side, the energy consumption and $\mathrm{CO}_{2} \mathrm{e}$ emissions by the grid electricity was $64.24 \mathrm{GWh}$ and $3.2 \times 10^{3}$ tonnes, respectively. Notably, a decade earlier when Ontario was reliant on coal, the GHG emissions from electricity supply to the university would have matched that of the CHP. The majority of buildings are assigned to the academic building category, which is associated with the highest energy demands with $50.5 \mathrm{GWh}$ accompanied by the highest $\mathrm{CO}_{2} \mathrm{e}$ emissions of $6 \times 10^{3}$ tonnes. The parking building recorded the lowest energy consumption as it consumes only electricity for lighting purposes. 
On the other side, the normalized energy consumption and GHG emissions per unit area are shown in Figure 3.6. The highest electricity consumption was assigned to the Life Science building as it includes large number of specialized laboratories, which supports experimental work in the biological, biochemical, and behavioral sciences. The Tennis bubble building recorded the highest natural gas consumption per unit area and per person for heating purposes, which serves four tennis courts with a high ceiling with a poorly insulated envelope. For the normalized $\mathrm{CO}_{2}$ e emissions per unit area, seven buildings exceeded the average carbon emissions of $83.8 \mathrm{~kg} \mathrm{CO} 2 \mathrm{e} / \mathrm{m}^{2}$ by the universities in Ontario due to the high consumption of steam for heating or for food preparation purposes. Normalized energy consumption and GHG emissions per person are shown in Figure 3.7. Some of the buildings recorded low resource consumption per unit area while recording high consumption per person such as NWRC and CTTC buildings due to low occupancy levels. The National Wildlife Research center (NWRC) recorded the highest electricity consumption and GHG emissions per person as it includes 15 laboratories, a $100 \mathrm{~m}^{2}$ greenhouse and plant growth chambers, and specimen storage facilities with three walk-in freezer rooms, which requires very low temperature varying from -40 to $-150^{\circ} \mathrm{C}$. While, for the CTTC building, high electricity consumption per person was recorded as it includes health services, pharmacy, and dental clinic. Most of the buildings are below the average energy intensity consumption by the university sector in Ontario. This is due to the climatic conditions of Ottawa being significantly colder than most other major population centers in the province.

There are different approaches to reduce campus GHGs emissions. District energy systems could also be utilized to pump steam, hot water, chilled water to multiple buildings 
on campus. The current steam system could be replaced by a cogeneration unit, which takes the advantage of thermal energy produced during electricity generation. The thermal energy could be used for different applications such as water heating, space heating and cooling (by linking cogeneration unit to absorption chillers that uses waste heat for refrigeration). Providing heating and cooling from central plant usually requires less fuel and displaces the need to install separate equipment in each building. Moreover, cogeneration units reduce GHGs by avoiding transmission and distribution losses that occur when electricity travels over power lines. However, there are two main issues in utilizing cogeneration units: 1) fossil-fuel based cogeneration units still emit a lot of $\mathrm{CO}_{2}$, and 2) cogeneration units that make use of low-temperature waste heat have much lower thermodynamic efficiencies than using high-temperature waste (as the quality of heat is lower than the quality of electricity) [184]. Moreover, a hot water system could also be used where renewable energy sources could be integrated into the system. The hot-water system has been proposed by UBC and it is expected to reduce GHGs by 33 percent accompanied by a reduction in operational and energy costs by $\$ 5.5$ million per year [185]. Another alternative is to apply air or ground source heat pumps to buildings using their own boilers. However, air source heat pumps have drawbacks when applied in cold climates, as they likely need supplemental heat source. On the building scale, electricity usage could be reduced by using high efficiency lighting and automated building controls for heating, air conditioning and ventilation systems. This study focused on calculating average GHGs based on the annual electricity mixture. If the temporal resolution was increased, this could help in identifying the electricity mixture by different power generation sources. This information, in turn, could inform policy makers about 
opportunities for energy savings and GHG emission reductions.

By comparing the consumption of each building having the same activity, it was noticed that building activities/functions have a more significant impact than building age upon energy consumption and $\mathrm{CO}_{2} \mathrm{e}$ emissions. This can be shown in the finding that some older buildings for residence, athletics, and academic seem to be comparatively better than newer buildings in terms of energy and water consumption. Despite that, older buildings while having less efficient envelopes and systems, they tend to have less associated plug loads than newer buildings. Moreover, occupant behavior and operational schedules also may have significant impact on energy consumption. Furthermore, as some buildings share the same chiller to provide cooling, thus cooling electricity allocation could not be provided for such buildings. Thus, more investigations regarding meter boundaries and more analysis on building level are required in future work. Moreover, further sub-metering the buildings would provide greater insight into building performance. The current practice is to have periodic energy audits [180]; but real-time data analysis is more effective for detecting operational inefficiencies and system failures.

The average district water, natural gas, and steam consumption in tonnes are shown in Appendix A. 1. Around 90 percent $\left(428.9 \times 10^{3} \mathrm{~m}^{3}\right)$ of district water is distributed to buildings for use in sinks, showers, drinking fountains, and laboratories, while the rest $\left(41.64 \times 10^{3} \mathrm{~m}^{3}\right)$ is supplied to the central heating plant to make up for water loss from the stream distribution system. On the building consumption side, it was recorded that the residence buildings consume the highest amount of water at $143.96 \times 10^{3} \mathrm{~m}^{3}$, due to 24 -hour occupancy and showering. 
For the normalized district water, natural gas, and steam consumption per unit area are shown in Appendix B. 1. The Gym building has the highest district water consumption due to the high usage of water for showers and serving a 50-metre $\left(\sim 2000 \mathrm{~m}^{3}\right)$ Olympicsized swimming pool. The Tennis Bubble building envelope is poorly insulated by which high natural gas consumption per unit area and per occupant is recorded as this building uses its own boiler to provide heating. As for steam consumption, Commons building has the highest consumption per unit area, as this building is the hub of residence life containing lounges, offices, a convenience store, and food court. While, the NWRC building recorded the highest steam consumption per person due to low occupancy levels in the building as shown in Appendix B. 2. Moreover, a high amount of steam consumption was recorded for the Life Science and Nesbitt buildings, which serves environmental laboratories, climatecontrolled greenhouses that contain large collection of plants for teaching and scientific study in Canada [186]. The residence buildings have high steam consumption; this is due to high setback temperatures due to 24-hour occupancy.

On the utility cost side, Appendix A. 3 shows average utility cost of $\$ 12.1 \mathrm{M}$. Around 62.8 percent of the utility budget was allocated to electricity, 23.2 percent to natural gas, and 14 percent to district water. Academic function records the highest energy consumption; this is due to the large number of buildings allocated to academic purposes versus other activities. Approximately $\$ 800,000$ per year was allocated to heat loss from the CHP due to inefficiency of the CHP and distribution losses through pipes.

The highest utility cost per unit area regarding steam, electricity, and district water were assigned for Commons, Life Science, and Gym buildings, respectively as shown in Appendix B. 3. However, Gym and Commons buildings recorded low utility cost per 
person, as it serves large number of occupants as shown in Appendix B. 4. Moreover, the NWRC building recorded the highest utility cost per person. Furthermore, buildings with multi-functions recorded high utility costs as they consume more amount of energy to serve different facilities such as restaurants/cafes. As pointed out earlier, building age does not have significant impact upon energy consumption.

The aim of the survey is to evaluate different visualization tools specialized in tracking building/campus energy and water use. The survey tackled some general questions regarding the current visualization tools and their weaknesses. Moreover, the type of analysis facility management and operation (FMP) employees and other property management companies perform were incorporated in the study. The survey then focused on the comparison between bar graphs and Sankey diagrams in terms of their effectiveness in visualizing building performance over existing visualization methods/tools, usefulness for facilitating decision-making, and in identifying abnormal behavior in energy and water consumption utilizing spatial and temporal analysis. The survey questions are mentioned in Appendix C. 1.

It was concluded that the current visualization tool is relying on bar graphs, line charts and simple spreadsheets. The current methods/tools are good for diagnosing problems and understanding how building controls are functioning. However, some limitations are associated with the current tools in terms of its limited capabilities. It also does not provide a good platform for understanding the overall performance of a particular building or mechanical system. The type of analysis FMP would perform includes weather normalization to adjust for savings on retrofit projects, energy demand analysis (annual, monthly, weekly, and hourly), budgeting, and mechanical systems as well as their control. 
It was found that Sankey diagrams are more useful in tracking underperforming buildings and to visualize overall campus performance and proportionality. Moreover, it helps to demonstrate energy-saving strategies at different levels. However, more data resolution is required (i.e. monthly, weekly, daily, and hourly) to inspect and to identify abnormal consumption for each building individually. Some of the candidates recommended having a percentage differentiation against expected baseline for a better comparison.

On the other side, it was found that the bar graphs are useful in determining the consumption and performance patterns for each building and utility. However, they are less useful for getting a high-level of understanding in terms of campus performance. Moreover, it was recommended to have more data resolution to identify abnormal trends.

In conclusion, bar graphs are easier to compare building performance and provide a better quantitative understanding. On the other hand, Sankey diagrams are better in visualizing the overall campus performance for the wider Carleton community and facilitating qualitative analysis.

\subsection{Summary and Future Work}

This research proposed the outline for a methodology for creating Sankey diagrams and other visualization methods to represent energy flows on a university campus level. The aim of this methodology was to facilitate a greater understanding of how each building performs and to identify underperforming buildings so that building operators and other stakeholders can investigate further. This study also demonstrates the upstream environmental and economic impacts of buildings' and campus performance. However, 
higher data resolution (i.e. hourly or monthly and by subsystem, such as lighting and computing) is required to assess why certain buildings underperform relative to others. Further sub-metering the buildings and more analysis on the building level regarding meter boundaries is required for more robust analysis (discussed in Chapter 3). Normalizing the data per area and per person is crucial analysis towards assessing the building performance, but must be taken within the context that certain university activities require considerable space and/or resource inputs. The current methodology could be integrated in the future into a software tool that can be linked to real-time readings from meters based on annually, monthly, and hourly data. Sankey diagrams are relatively a simple means to visualize complex energy flows; however, the creation process was performed partially manually.

Future steps for this research initiative include:

- Developing computer tool(s) for automating the process that creates a Sankey diagram from campus sub-meters input file;

- Creating a Sankey diagram that can represent annually, monthly, and hourly data (discussed in Chapter 7);

- A software-based user interface that allows a designer to inspect instantaneous heat transfer and power use in a building at any given time (discussed in Chapter 7);

Moreover, more analysis on building scale is required in the future including calibrated models for better understanding building performance (discussed in Chapter 4). This includes:

- Heat gain sources from people, equipment, lights, and solar gains; 
- Heat gain/loss from conduction through envelope, short-wave radiation exchanges between indoors and outdoors, and ventilation and infiltration rates;

- Applying the methodology to multi-zone building using calibrated models. 


\section{Chapter 4: Data Visualization and Analysis of Energy Flow on a Multi-Zone Building Scale}

This chapter was submitted as:

\section{(Abdelalim A., O'Brien W. and Shi Z., "Data Visualization and Analysis of Energy Flow on a Multi-Zone Building Scale," Automation in Construction)}

This chapter proposes an integrated framework to estimate and visualize energy flows and the associated cost on the building level to provide a comprehensive energy use assessment that could be generalized to other existing modern commercial buildings to facilitate the decision-making by building operators.

\subsection{Abstract}

Modern commercial buildings' resource consumption is metered at various levels of spatial and temporal resolution to track and reduce energy use and greenhouse gas emissions. However, not all data that could be used to detect faults or identify efficiency improvements are available due to the cost of meters. In the field of building operation, building performance simulation (BPS) could help in quantifying unmeasured energy flows, for instance solar gains, heat loss from infiltration, etc. Furthermore, integrating building information modeling (BIM) in building operation and maintenance can decrease operation risk and costs, as well as maintain facility management quality. However, in practice there is a lack of efficient utilization of this application by building operators. Current data availability and many visualization tools do not lend themselves well to identifying inefficiencies and possible solutions. This research proposes an integrated framework to estimate and visualize energy flows and the associated cost on the building level to provide a comprehensive energy use assessment that could be generalized to other 
existing modern commercial buildings to facilitate the decision-making by building operators. The framework consists of 1) develop BIM model, 2) convert BIM to BPS, 3) calibrate model, and 4) visualize energy flow and cost using Sankey diagrams. The proposed method is then applied to a medium Canadian university building. Finally, the results of a survey that was deployed to a sample user group to assess usability of the Sankey diagrams are provided.

\subsection{Introduction}

Most of modern buildings utilize building energy management systems (BMS) for monitoring and optimizing building systems during operation. However, the data from metering and logging systems are often inconvenient and difficult to access due to use of multiple systems and technologies of varying vintages and platforms. BMS end-users (such as building operators and other stakeholders) might look for easily understandable metrics such as electricity cost rather than delivered energy [187]. However, the complex interactions between building systems make it difficult to understand the impact of changing a single component or operational schedule within a system [188]. For instance, the choice of light bulbs/fixtures should incorporate their impact on heating and cooling loads. Moreover, some other variables are impractical and difficult to meter. Building performance simulation (BPS) tools can be used to help in quantifying unmeasured energy flows, for instance solar gains, heat loss from infiltration, etc.

Building performance simulation has emerged as a viable method to emulate reality and improve on traditional manual methods to study and optimize the energy performance of buildings and systems $[35,34]$. In order to use the building performance simulation models to help in understanding the thermal behavior of an existing buildings, it should be 
calibrated with measured data. Several studies highlighted great discrepancies between simulated building energy performance and measured performance [189, 190]. Such discrepancies may be attributed to an incomplete knowledge of the building; the building model may thus not correctly reflect the real behavior of the building intended to be simulated [72]. This is due to lack of information about building's construction quality, occupant behavior (i.e. window openings, set point temperature, and internal gains), actual materials/equipment used, deterioration of building systems, and infiltration and ventilation rates, and algorithms uncertainties [191, 192]. Thus, adjustment of the model parameters is generally needed when applying a simulation tool to a real case on different levels of analysis (inspection/audit, evaluation of energy conservation opportunities (ECO’s) or continuous performance analysis) [100].

In the field of building operations, using building information modeling (BIM) in building performance analysis can facilitate a more accurate and efficient analysis process. Building information modeling (BIM) is a process involving the generation management of digital representations of physical and functional characteristics of building [8]. In order to perform a successful BIM-based building performance analysis, it is necessary to improve the interoperability between a BIM based architectural model and analysis programs [9]. Interoperability can occur either, directly between the platforms and tools, or using a data transfer model such as Industry Foundation Class (IFC) or the Green Building XML schema (gbXML) [24]. The initial BIM model is an architectural view of a building, and it does not necessarily correspond to a 'thermal' view necessary for performance analysis tools [193, 194]. Thus, BIM models should be adjusted first before data can be exchanged between a BIM platform and energy tools $[195,196]$. 
Most of the developed visualization tools for building energy management use simple line or bar charts for presenting and comparing metered energy consumption, greenhouse gas (GHG) emissions, and other performance metrics [14]. On the building level, Pulse Energy developed an Energy Dashboard tool that shows real-time building consumption of energy, natural gas, hot water, chilled water, and steam for campus buildings [143, 142]. Other tools such as Building Dashboard and Energy Efficient Education Dashboard were developed to visualize energy consumption of commercial buildings [145]. Yarbrough et al. [14] developed a new visualization tool on the campus level to understand the relationship between individual building peak demand and the campus peak energy use based on data provided by energy meter and billing peak. Current visualization tools or models typically only provide an end-use breakdown of energy consumption, depending on installed meter resolution. However, these tools are difficult to provide a comprehensive energy flow analysis on the building scale (i.e. how energy enters and is consumed in buildings). Furthermore, the current data availability and visualization tools do not lend themselves to identification of inefficiencies and possible solutions to improve efficiency or recover energy.

On the other hand, Sankey diagrams can provide relative flow magnitudes, direction of flows, inputs and outputs of interacting systems, energy recovery, and spatial representation (e.g., the layout systems and components can be approximately laid out in a Sankey diagram). Abdelalim et al. [197] proposed several methods to analyze and visualize building-level water, natural gas, and electricity consumption and the upstream environmental impacts and the associated cost using Sankey diagrams and other graphical techniques. Belzer [198] developed energy flow maps to depict energy flows from source 
to end-use in the building sector using Sankey diagrams. The end-use consumption was based on estimations from Building Energy Data-book (BED). Another application by Phineas [199] used dynamic Sankey diagrams to visualize internal and external flows through building envelopes. The study by Phineas helped in visualizing the amount of energy hitting and leaving the façade by radiation and convection. The proposed Sankey diagrams by Phineas also helped in visualizing the amount of energy required for heating and cooling to maintain an acceptable indoor air temperature. O'Brien [151] addressed major issues involved in creating Sankey diagrams to represent building energy flows of a solar house obtained from a building performance simulation (BPS) model. Schlueter and Thesseling [8] developed a prototypical tool integrated into building information modeling (BIM) to enable instantaneous energy and exergy calculations. Moreover, Sankey diagrams were implemented in the proposed tool to visualize the resulting performance indices [8]. A limited number of building design and analysis tools, such as CASAnova [154] and Sefaira [155] use Sankey diagrams for visualizing predicted energy use.

This study proposes a method to analyze and visualize energy flow and the associated cost using Sankey diagrams. Within the scope of this work, by using a combination of measured and model data, a comprehensive energy use assessment at the building level can be formed. This information can yield greater insights about opportunities for operational improvements and retrofits that would not be available through measurements alone to facilitate the decision-making by building owners, operators, and other stakeholders. This work contributes to the state-of-the-art by developing an integrated framework using measured and modeled data to estimate and visualize energy flows and associated costs on the building-level that could be generalized 
to other existing modern commercial buildings. The framework consists of 1) develop BIM model, 2) convert BIM to BPS, 3) calibrate model, and 4) visualize energy flow and cost using Sankey diagrams. Moreover, this approach will result in estimating and understanding the impact of unmeasured energy flows (for instance, solar gains, heat loss from infiltration, etc.). The proposed method is then applied to a case study: The Canal Building at Carleton University in Ottawa, Canada to assess the effectiveness of the proposed Sankey diagrams. Finally, the results of a survey that was targeted at building energy professionals to assess the effectiveness of the sample Sankey diagrams are presented.

\subsection{Framework}

The framework of this study is divided into three main parts. The first part consists of: 1) develop BIM model and convert the BIM-based architecture model to a building performance model, 2) perform model calibration, and 3) modeling approach in energy analysis tool, which are discussed hereunder. The second part of this framework converts model data into reliable energy flows and costs and produces Sankey diagrams. This is discussed in Section 4.5 after presenting the case study. The third part tests the effectiveness of Sankey diagrams through a survey. Figure 4.1 shows the proposed framework of this study. 


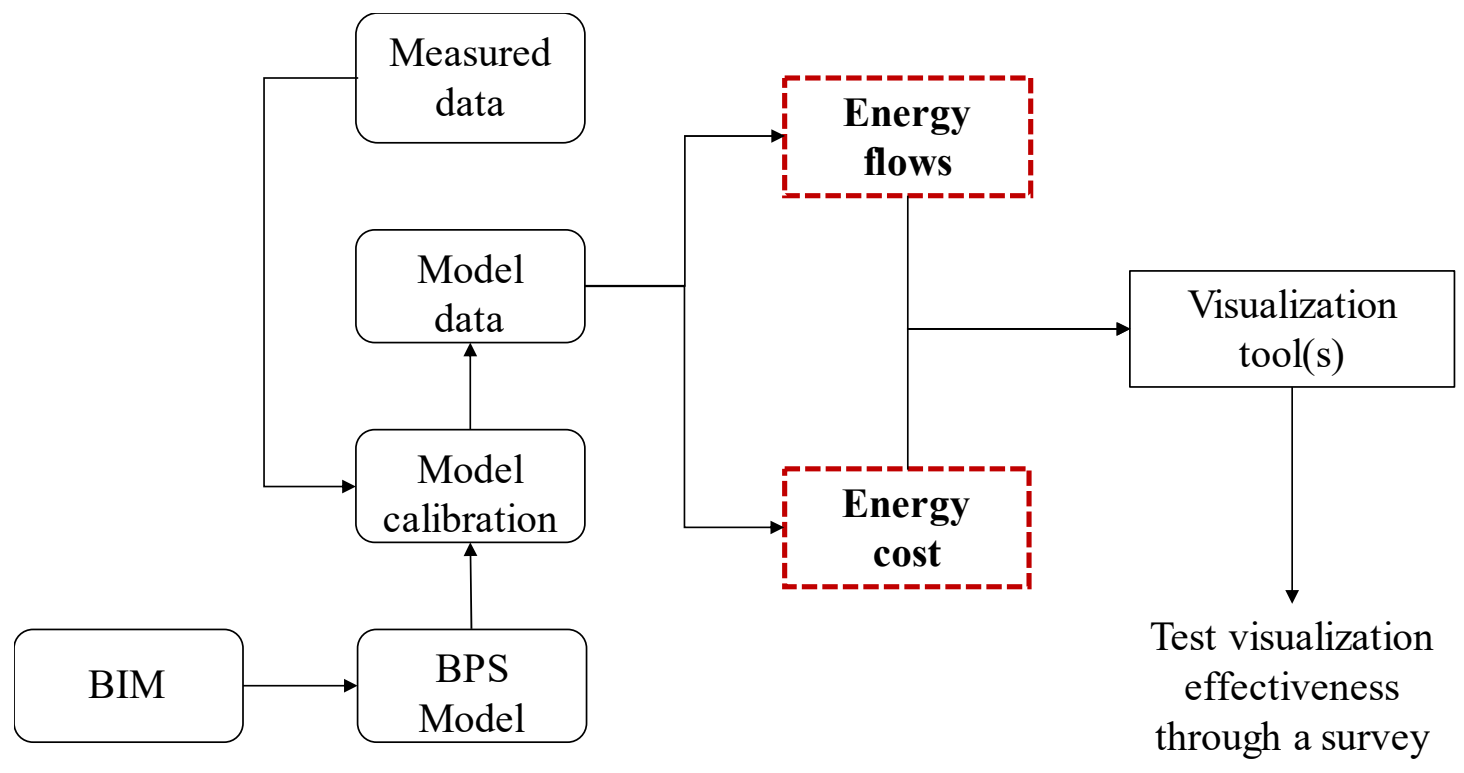

Figure 4.1: The proposed framework

The building energy management systems (BMS) provides real-time and historical energy consumption data, which are obtained from meters and sensors for some components such as lighting, equipment/appliances, elevators, air handling units (AHUs) components, VAV (variable air volume)-reheat coils, radiant panels, chillers, pumps, and steam. However, some other variables are impractical and difficult to meter such as internal gains, solar gains, infiltration rates, opaque envelope heat loss, and window heat loss as shown in Figure 4.2. These variables can be approximated using a calibrated building performance simulation (BPS) model based on many modeling assumptions and simplifications. EnergyPlus 8.1 was selected as the BPS tool due to its technical documentation and versatility and capability of simulating complex building systems [200]. On the other hand, building information modeling (BIM) tools could provide detailed information about building geometry, construction, space and zone, material properties, operational schedules, equipment and lighting power densities that could be used in BPS tools. These data can be obtained from the available as-built documents, BMS, 
and from on-site audit. Enhancing the conversion process from BIM to BPS should eliminate data repetition and inherent human error.

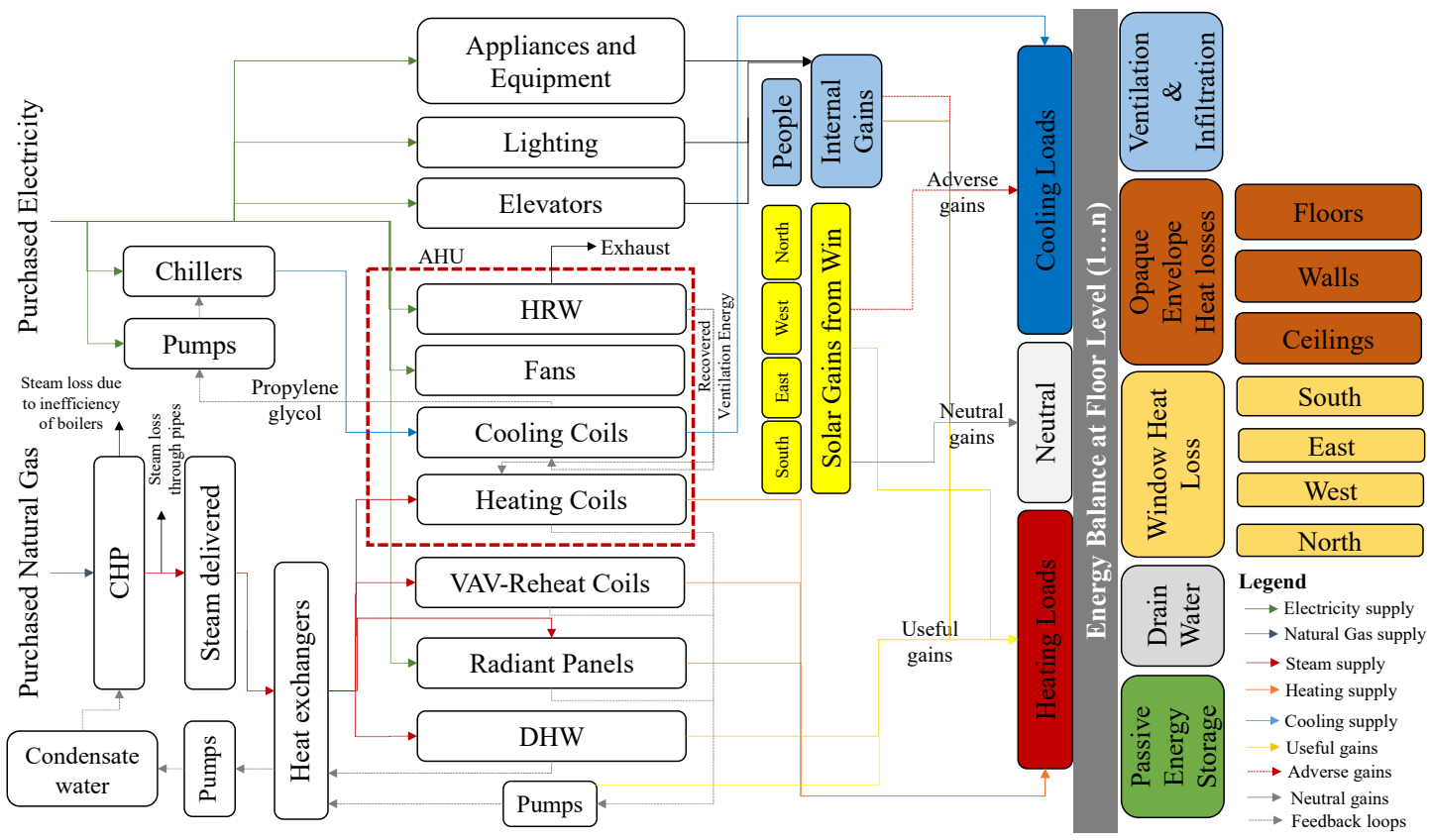

Figure 4.2: Flowchart showing the main scope of the study on the building level

\subsubsection{Convert BIM based Architectural Model to Building Performance Model}

In this study, Autodesk Revit 2016 software was used for the model development process. Figure 4.3 illustrates the entire building model development process. To achieve interoperability between BIM and building performance simulation (BPS) tools, the gbXML file format was selected for modeling and data storage as it facilitates the exchange of data among BIM and energy analysis tools [30]. The first step was to identify the level of details required to achieve a smooth transition between the BIM model created in Revit to the BPS tool (Energyplus in this case). This was a crucial step to avoid high polygon models that may not be produced in the gbXML [32]. 


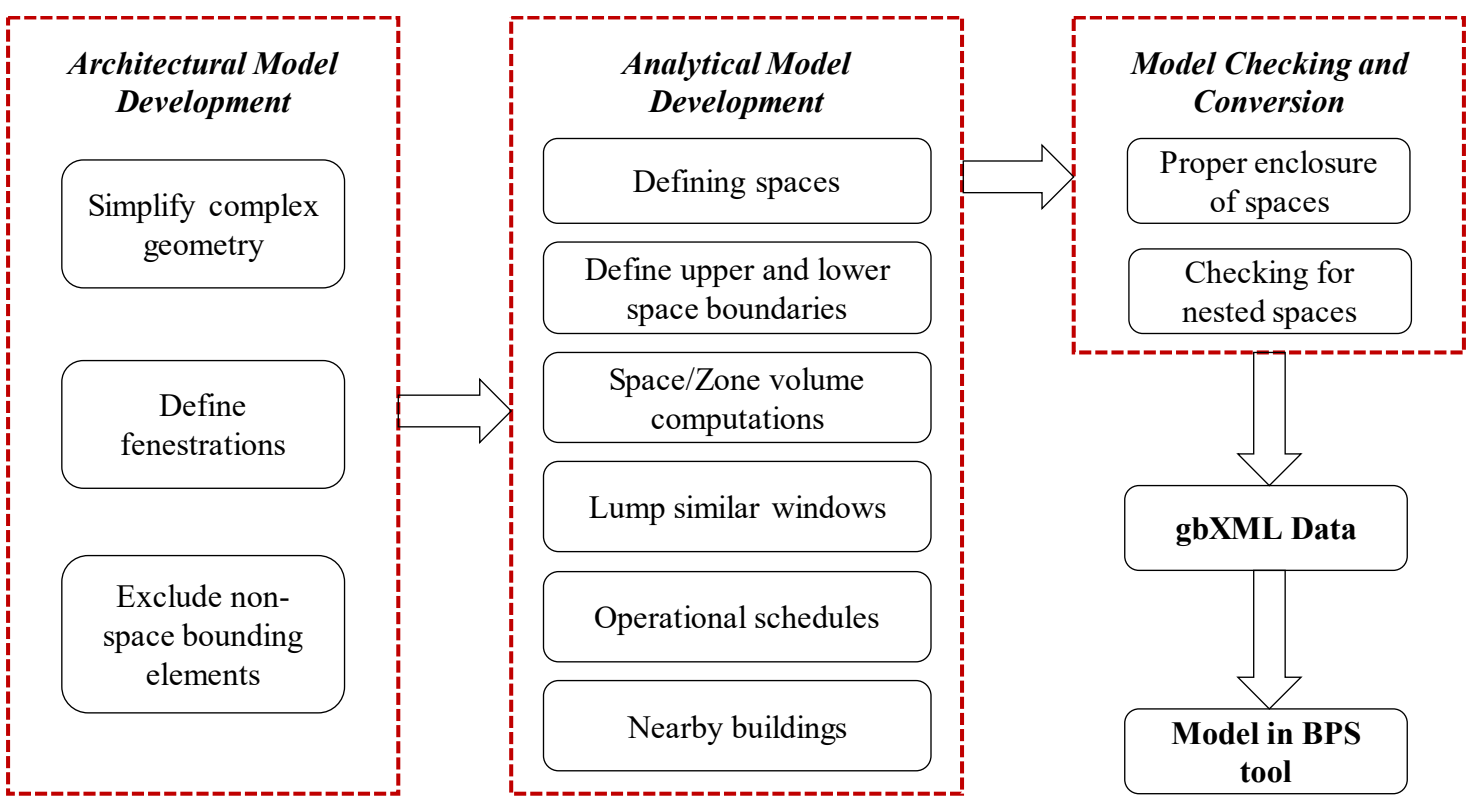

Figure 4.3: Building Model Development Process

This section demonstrates the process in developing the BIM architectural model:

1) Simplify complex geometries: the first step was to simplify the model to prevent errors and long simulation time that could be caused by building components with irregular or complex surfaces or having large number of surfaces.

2) Define fenestrations: in energy analysis tool (EnergyPlus in this case), fenestration is modeled as sub-surfaces (i.e. windows/doors inside a wall). However, in case of having a curtain wall covering the whole façade, Revit tool model the curtain wall as a single element. Thus, the curtain wall elements were converted to windows with equivalent thermal and optical properties.

3) Exclude non-space bounding elements: some elements such as structural columns could have thermal mass effect, however, it is insignificant compared to the overall thermal performance of spaces/zones in large commercial buildings. Thus, structural elements were removed from the energy model. Furthermore, their removal could avoid problems 
regarding computation of zone area and avoiding complex surfaces in the energy model to reduce the simulation time.

The following steps are taken to prepare the analytical model:

1) Define spaces: a successful analytical model could be obtained if all the spaces in the model are defined by physical elements. However, in case there is a large space that is controlled by different HVAC terminal units or hydronic systems, space boundaries can be manually defined properly to separate the space into appropriate thermal zones. On the other hand, spaces with the same boundary conditions, HVAC system, same heating and cooling set points and thermostat schedules, or internal gains schedules, can be grouped, taking into account separating perimeter volumes from internal volumes.

2) Define upper and lower space boundaries: in energy analysis tool (EnergyPlus in this case), the space dimensions are defined from the interior surface. Thus, the upper and lower boundaries were modified to reflect the interior surface of each space.

3) Compute space/zone volumes: areas and volumes of spaces should be calculated as it is not default in some BIM tools such as Revit. This will help in determining the volume of each space having different heights.

4) Lump similar windows: similar windows on surfaces were lumped to simplify the model.

5) Input operational schedules: schedules for lighting, plug loads, and occupancy were also added based on the available data to the gbXML file.

6) Input nearby buildings: only nearby buildings were modeled as blocks (i.e. without details), as they will affect shading on the building. 
7) Define HVAC: HVAC system information in gbXML produced by Revit cannot be imported to EnergyPlus. Thus, HVAC data was excluded from gbXML and was modelled using Green Building Studio (GBS).

It is important to make sure that the model is correctly configured for exporting throughout the following checks:

1) Properly enclose of all spaces: spaces in the BIM model must have a properly enclosed region to be translated to energy analysis tools. In case of failing to identify a space in BIM model, it is crucial to check for disjoint surfaces to be able to fix surface boundary lines.

2) Check for nested spaces: the nested spaces are useful when defining a space within a space in BIM model. However, as gbXML format does not support such feature. Thus, spaces with such situation were manually defined using space boundary lines.

\subsubsection{Model Calibration}

The aim of the model calibration in the proposed framework was to obtain greater data about building performance and to supplement measurements. This study proposes a hybrid evidence-based and analytical optimization method that has been developed by Coakley et al. [201]. Figure 4.4 shows the proposed calibration methods. Modern buildings are usually equipped with numerous sensors and sub-meters. However, in most cases, information regarding number and types of equipment used in each zone are not documented and there is no sub-metering in individual rooms. Thus, an on-site audit is required to obtain such information. Building operation data for HVAC loop components (i.e. pumps, chillers, cooling towers, boilers, and heat exchangers), air handling unit (AHU) sub-systems (i.e. outdoor air fraction, supply temperature and relative humidity, and flow 
rates), air distribution system, radiant panels, and zone setpoint temperatures could be obtained from the building energy management systems (BMS). In the context of the selected case study, information regarding occupancy schedules were obtained from occupancy sensors installed in private offices and from a university database. However, the actual number of occupants in classrooms or laboratories is not measured. Furthermore, AMY (actual meteorological year) data was used for Ottawa, Canada to obtain weather data based on the NOAA/NCEP Climate Forecast System Reanalysis (CFSR) model [202].

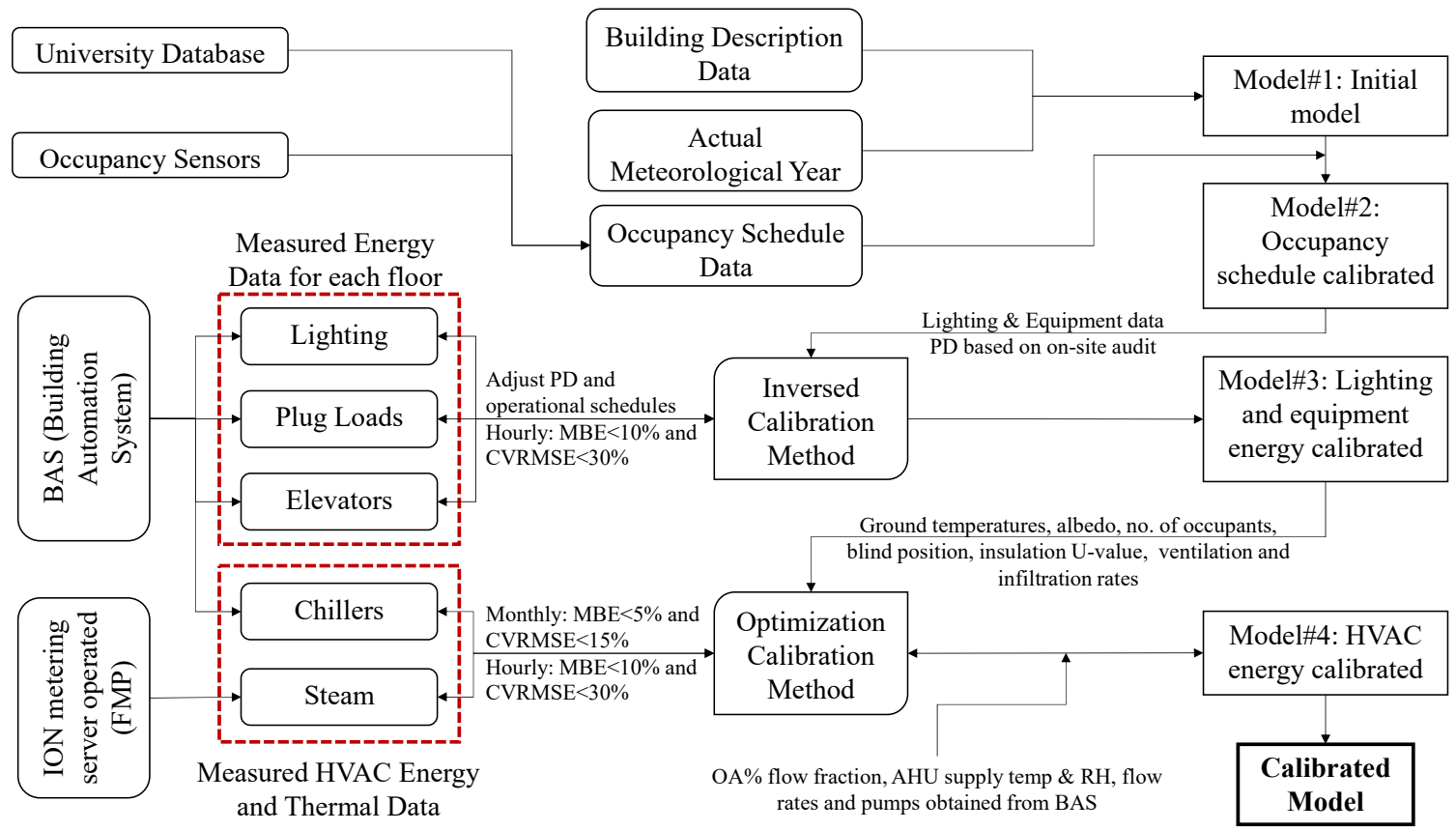

Figure 4.4: The building energy model calibration method to achieve (MBE) of $\pm 10 \%$ and CV (RMSE) of $\pm 30 \%$ for hourly based calibration, as well as achieving (MBE) of \pm $5 \%$ and $\mathrm{CV}$ (RMSE) of $\pm 15 \%$ for monthly based calibration

In the context of the selected case study, the data obtained from building energy management systems (BMS) included only consolidated lighting, equipment, and appliances electricity consumption data for each floor. However, building performance simulation (BPS) tools such as EnergyPlus require hourly schedules for each zone. In this study, an inverse calibration procedure was developed to tune lighting, equipment power 
densities and schedules based on the proposed method by Lam et al. [61]. A Matlab script was written to automate the process of obtaining hourly schedules for each zone as shown in Figure 4.5. The method follows six steps: 1) assumed power density based on on-site audit for each room in the building; 2) constraints are set for operational hours based on on-site observations; 3 ) hourly computed power for lighting and equipment are compared to actual measured data to meet the Mean Bias Error (MBE) and Coefficient of Variation of the Root Mean Square Error CV(RMSE) criterion for each floor; 4) if the criteria are not met, based on the room type (hallways, offices, classrooms, teaching labs, café, or mechanical rooms), an hourly inverse calibration factor, calculated by taking the hourly measured power $(\mathrm{P})$, divided by the computed power (P-simulated) taking into account operational hours, are multiplied by the hourly schedule; 5) the calibrated power densities are calculated by multiplying the calibration factor by the assumed lighting and equipment densities; and, 6) if the criteria are met, an 8760-hour schedule including weekdays, weekends, and national holidays is generated to input in EnergyPlus. 
Adjust PD based on room type and equipment

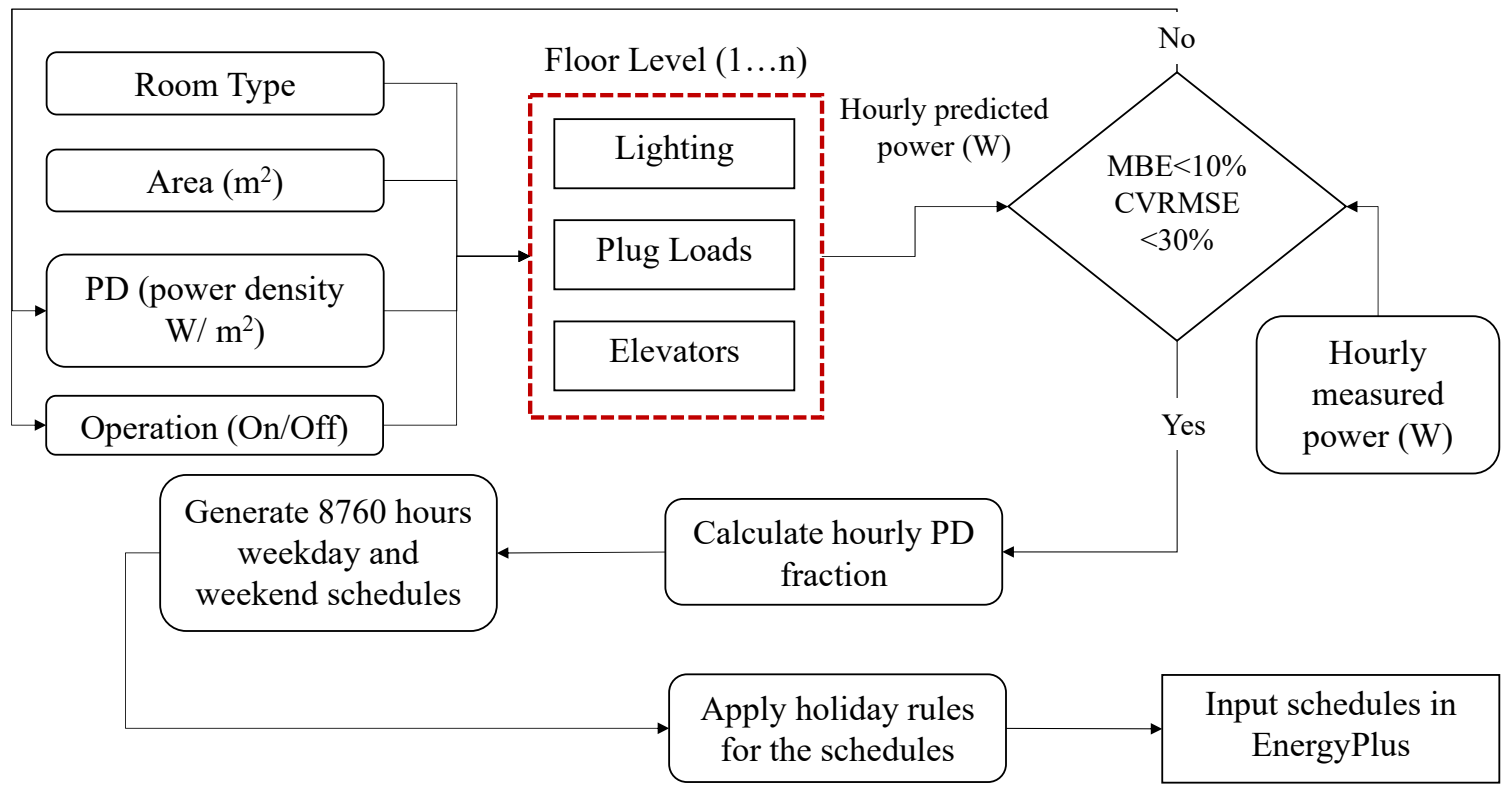

Figure 4.5: The inverse calibration procedure

Vast amounts of information were obtained from building energy management systems (BMS) and as-built documents. However, in the context of the selected case study, some parameters were difficult to obtain and have significant impact on the building performance, such as the actual properties of the insulation materials, percentage of operable window openings, blind positions, ground temperatures, ground albedo, number of occupants, and ventilation and infiltration rates [191, 201]. Thus, the optimization method was applied to help in identifying a set of input parameter values that minimize an objective function during the calculation of simulation cases. The requirements as recommended by ASHRAE $14-2002$ is to achieve (MBE) of $\pm 10 \%$ and CV (RMSE) of \pm $30 \%$ when calibrating hourly data. While, (MBE) of $\pm 5 \%$ and CV (RMSE) of $\pm 15 \%$ is required for monthly based calibration.

The ExCalibBEM tool developed by Hydro Quebec was used in order to facilitate 
building model calibration to measured data [203]. This tool is a graphical interface for GenOpt [90]. The optimization algorithm used in this study is Generalized Pattern Search algorithm (GPSHookeJeeves) which is useful to obtain optimal solution versus the number of evaluation when dealing with continuous parameters. The initial values of the parameters modified, were taken from the available drawings and specification documents and are discussed in Section 4.4.2.

\subsubsection{Modeling Approach in EnergyPlus (E+)}

One of the crucial steps that has to be considered is the modeling approach used in energy simulation tools (EnergyPlus in this case) [74]. EnergyPlus uses the "Heat balance" method in determining zone thermal response, by which room air is modeled as well mixed with uniform temperature throughout each zone. The heat balance model is also applied to external and internal surfaces, where room surfaces have uniform surface temperatures, uniform long and short wave irradiation, diffuse radiating surfaces, and internal heat conduction. The following discussion covers the modeling approaches chosen for the current case study.

For conductive heat transfer, CTFs (conduction transfer functions) for transient conduction through the opaque envelope was selected, as the material's thermo-physical properties were assumed to not vary with temperature. For the inside convection algorithm, as not all zones have the same configurations for diffusers in the selected case study, thus US-DOE [33] recommends using the adaptive convection algorithm developed by Beausoleil-Morrison [204]. This method provides a dynamic selection of convection models based on the space conditions and configurations. The same algorithm was selected for the outside convection algorithm, which provides finer control for different categories 
for surfaces that depends on current wind direction and heat flow directions.

For diffuse sky radiation, EnergyPlus uses the anisotropic sky model by Perez et al. [205]. EnergyPlus has the option to define different monthly values for albedo. For window properties, Window LBNL was used to obtain values for SHGC, U-value, solar and visible transmittance, and spectral data. For the transmitted beam radiation, the default model was selected for the case study, as some zones in the model are non-convex zones. The assumption in the default model is that all transmitted beam radiation strikes the floor, some is absorbed based on the absorptivity of materials, and some is reflected based on an area weighting. Moreover, shading (aka shadowing) is calculated by default every 20 days. Of the diffuse irradiance, only that from the sky is determined, while shading of groundreflected diffuse irradiance is not considered in simulations. For view factors between internal surfaces, EnergyPlus calculations are determined by area weighted and also limited to "seen" surfaces.

For the infiltration model, infiltration design flow rate model was selected. In this model, the user defines a design flow rate that can be modified by temperature differences and wind speed. Infiltration is specified as a design level, which is modified, by a schedule fraction based on temperature difference and wind speed. Thus, it is not constant over the whole year. The ventilation design flow rate model was selected to calculate the ventilation rates. This model enables the user to define a design volume flow and schedule fraction. The actual flow rate of ventilation can be modified by the temperature difference between the inside and outside environment and the wind speed.

For the ground heat transfer model, US-DOE recommends using the advanced 
method (3D ground heat transfer) if the building is very small, otherwise it will not be significant [33]. The Canal Building is $8,000 \mathrm{~m}^{2}$ and consists of one unconditioned basement for which only a small portion is below grade. Thus, FCfactor Method for ground heat transfer was selected.

US-DOE recommends time steps of ten minutes in the case of building simulation models that include heating ventilation and air conditioning (HVAC) [33]. A detailed HVAC system was modelled in DesignBuilder. The detailed HVAC model enables definition of air loops, plant and condenser loops, HVAC zone groups, system control and set point managers, and sizing of HVAC flow rates. Both the heating water and chilled water distribution loops in the building were modeled as variable flow systems. A sequential distribution scheme was also selected as it operates equipment in a serial manner based on the loop operation scheme (which assigns priority to different equipment on the loop). When the highest priority equipment is at capacity, the next highest priority equipment tries to meet the load, etc. For modeling the hot water plant loop, it is required to model boiler and specify its efficiency. In the context of the Canal Building, the central heating plant (CHP) produces steam delivered to the building. Thus, two work-around modeling options are available: either by using district heating or using a high boiler efficiency. The district heating is only available in simple HVAC models, thus a boiler with high (100 percent) efficiency was selected.

\subsection{Application: The Canal Building}

The Canal Building was selected for the case study because it includes a large variety of functional spaces such as private offices, open-plan offices, lecture rooms, computer labs, design labs, research labs, conference rooms and other facility rooms. The 
building is also equipped with more than 2500 sensors to collect data required to inspect sub-hourly energy consumption for each floor. The building began operating in 2011 and its key characteristics are provided in Table 4.1. The current study focused on historical data for four months in winter (January-April 2015) and summer (May-September 2015). This study aimed at analyzing the building with a definitive heating and cooling energy season changeover (i.e. excluding shoulder seasons).

Table 4.1: Building details summary

\begin{tabular}{|c|c|c|}
\hline General & $\begin{array}{l}\text { Floor Area }\left(\mathrm{m}^{2}\right) \\
\text { No. of stories (including basement) } \\
\text { No. of modelled thermal zones } \\
\text { Bldg. type } \\
\text { Window to wall ratio (WWR): } \\
\text { - North }\left(315 \text { to } 45^{\circ}\right) \\
\text { - } \quad \text { East }\left(45 \text { to } 135^{\circ}\right) \\
\text { - South }\left(235 \text { to } 225^{\circ}\right) \\
\text { - } \quad \text { West }\left(225 \text { to } 315^{\circ}\right)\end{array}$ & $\begin{array}{c}8,000 \\
7 \\
107 \\
\text { Mixed-use academic }\end{array}$ \\
\hline $\begin{array}{c}\text { Building } \\
\text { Envelope } U \text { - } \\
\text { value }\left(W / m^{2} . K\right)\end{array}$ & $\begin{array}{l}\text { Roof } \\
\text { Walls } \\
\text { Foundation walls } \\
\text { Intermediate floor } \\
\text { Windows (double-glazed with air gaps of } 13.5 \mathrm{~mm} \text { ) } \\
\text { Window (SHGC) }\end{array}$ & $\begin{array}{c}0.14 \\
0.24 \text { to } 0.47 \\
0.1 \\
2.5 \\
2.67 \\
0.722\end{array}$ \\
\hline HVAC air loop & \multicolumn{2}{|c|}{$\begin{array}{l}\text { Two small air-handling units (AHU) are designated for the mechanical } \\
\text { rooms, while, the rest of the building is conditioned by two separate } \\
\text { identical AHU units. } \\
\text { Single duct VAV-box; non-corner spaces' VAV boxes contain reheat coils }\end{array}$} \\
\hline Chiller & \multicolumn{2}{|c|}{ ElectricEIRChiller Centrifugal Carrier 19XR 1407kW/6.04COP/VSD } \\
\hline Space heating & \multicolumn{2}{|c|}{$\begin{array}{c}\text { Relies on the steam generated at a central plant. Some perimeter rooms are } \\
\text { equipped with radiant panels }\end{array}$} \\
\hline $\begin{array}{c}\text { Thermostat } \\
\text { settings }\end{array}$ & \multicolumn{2}{|c|}{$\begin{array}{l}\text { heating / cooling setpoints are variable (ranges from } 20^{\circ} \mathrm{C} \text { to } 24^{\circ} \mathrm{C} \text { ). During } \\
\text { that period, there were no setback temperature installed }\end{array}$} \\
\hline
\end{tabular}

\subsubsection{Building Performance Model Development}

The gbXML file exported from BIM based on the building model development process mentioned in Section 4.3.1 was then imported to DesignBuilder (a graphical 
interface tool for EnergyPlus). EnergyPlus simulation results output files were used to check for model functionality; errors and warnings. Figure 4.6 shows the model development for the case study (The Canal Building) from building information modeling (BIM) to building performance simulation (BPS). Figure 4.7 shows different zone activities and boundary conditions for the fourth floor (as an example) of the Canal Building. Zones with the same activity, boundary condition, heating ventilation and air conditioning (HVAC) system, same heating and cooling set points, thermostat schedules, and internal gains were merged together.
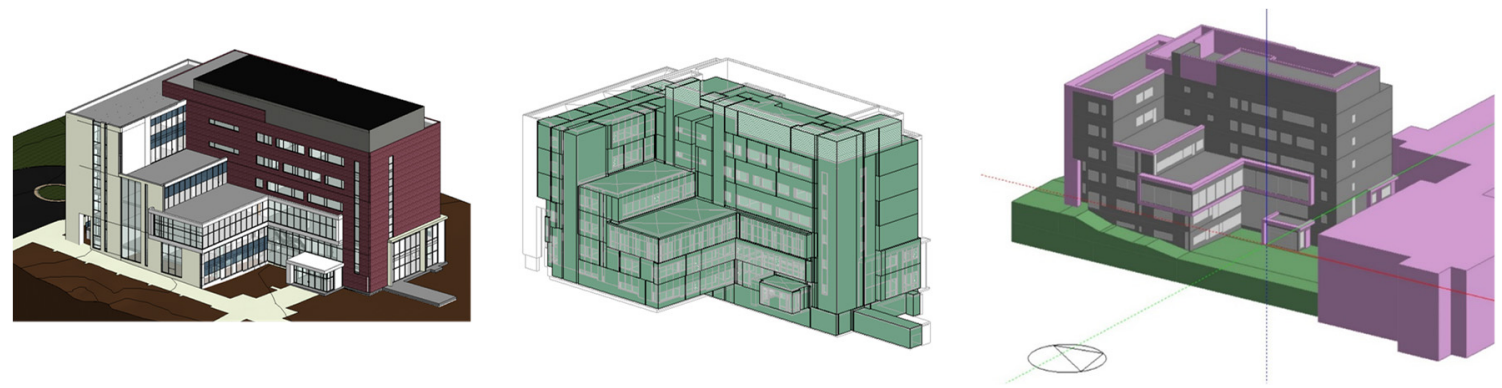

Figure 4.6: The conversion process from BIM (left) to analytical model (middle) to BPS tools (DesignBuilder) (right) 


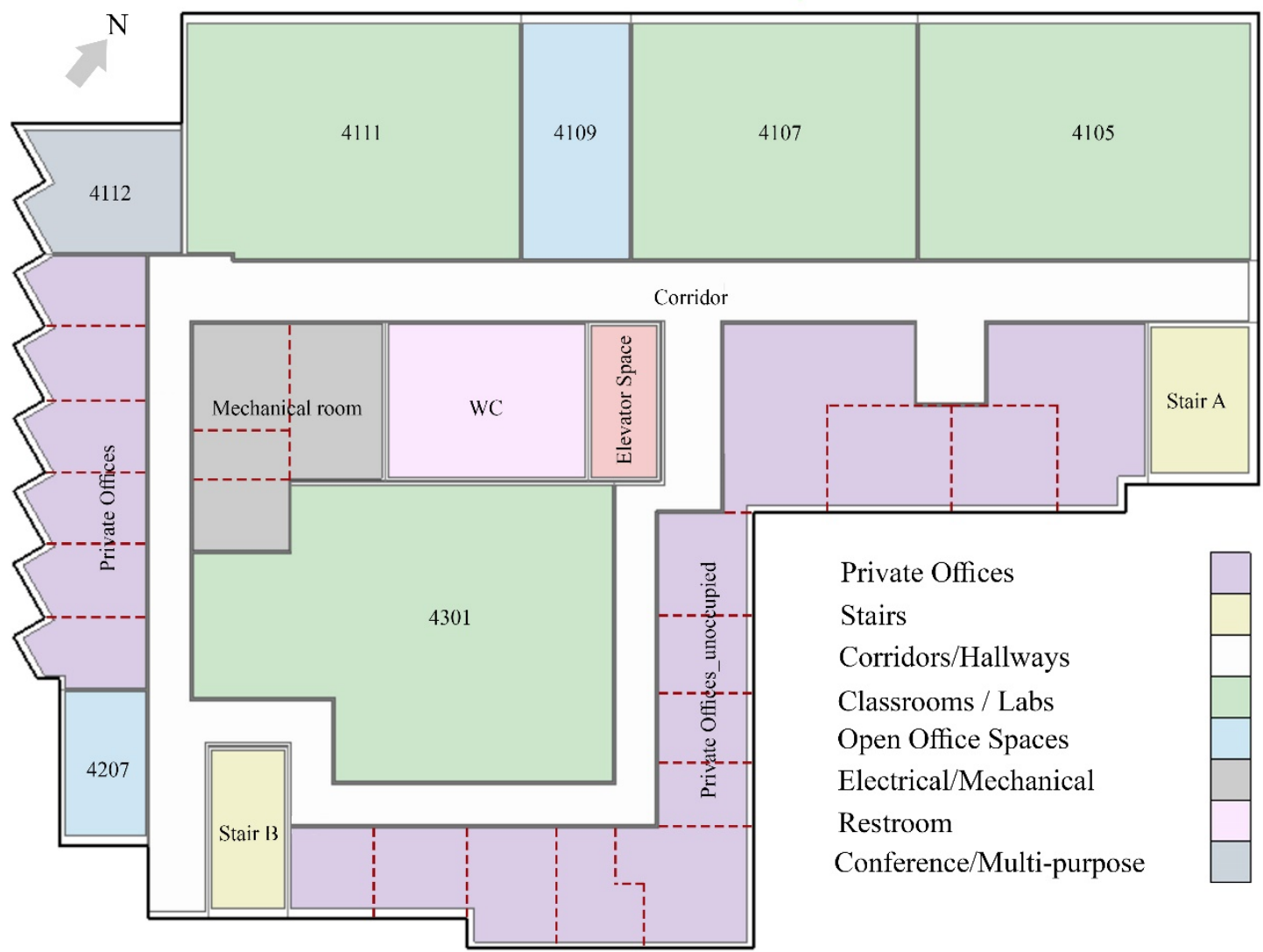

Figure 4.7: The Canal Building fourth floor showing different zone activities and merged zones (same activity and boundary conditions) within dotted lines

\subsubsection{Model calibration Results}

This section aimed at showing the model calibration results based on the proposed calibration method illustrated in Section 4.3.2. The modified parameters used in the optimization calibration method were obtained through measurements, on-site audit or further research and are discussed hereunder.

The ventilation opening area refers to a fraction representing the effective area of the total operable window area available for natural ventilation ranging from 0 to 1 with an increment of 0.05 [191]. An initial value of 0.3 was selected for the initial model from onsite audit. The number of occupants in each classroom was represented by a fraction ranging from 0 to 1 with an increment of 0.1 of the total capacity of each classroom [191]. 
An initial value of 0.4 was selected for the initial model from on-site audit. The albedo (reflectance of the ground surface) value ranges from 0.13 to 0.26 for climates without snow and 0.5 to 0.7 in the presence of snow according to Thevenard and Haddad [206]. An initial value of 0.26 with a value of two for snow modifier according to the default values of EnergyPlus was selected. The blind position refers to a fraction representing the effective area available for blocking direct solar radiations ranging from 0 to 1 with an increment of 0.1 . An initial value of 0.4 was selected from on-site audit. For infiltration rates, different studies in the literature have mentioned that a range between 0.05 and 0.8 Air-Change-Per-Hour ( $\left.\mathrm{ACH}_{50} \mathrm{pa}\right)$ is usually used for office buildings [71, 207, 208]. An initial value of 0.3 and 0.05 was selected for perimeter and internal zones, respectively, and was modified with an increment of 0.01 . For the thermal conductivity and specific heat of the insulation materials, the work of MacDonald [208] was taken as a basis, which considered the sum of the systematic uncertainties (differences in temperature, humidity and age of the material). An initial value for thermal conductivity and specific heat of 0.03 (W/m.k) and $960(\mathrm{~J} / \mathrm{kg} . \mathrm{k})$, respectively, was selected from the construction drawings. This value was modified with an increment of 0.001 and 10 for thermal conductivity and specific heat, respectively. For ground temperature, standard deviation of $4^{\circ} \mathrm{C}$ for each month of the year was considered, based on the CWEC (Canadian Weather for Energy Calculations) weather file [191]. The initial values for ground temperatures were obtained from Energyplus based on $\mathrm{F}$ and $\mathrm{C}$ factors of the underground wall constructions and slabs-ongrade or underground floors.

Some modest errors were associated with some of the sensors such as missing or incorrect data points. Thus, for short time-periods ( $<6$ hours), it is recommended to use 
simple interpolation to generate missing values. While for long time-periods, the data was excluded from the analysis [201].

Calibration results showed that lighting energy and plug loads have good agreement with the measured data. Hourly MBE and CV (RMSE) for lighting energy were $0.03 \%$ and $0.07 \%$, respectively. While, for plug loads, hourly MBE and CV (RMSE) were $0.02 \%$ and $0.05 \%$, respectively. On the other hand, the heating and cooling loads showed less agreement with measured data. This is due to uncertainty of occupant behavior (i.e. leaving windows opened), multiple setpoint temperature schedules, different operational schedules for VAV-preheat coil and radiant panels, and modeling assumptions and errors. Monthly MBE and CV (RMSE) for heating loads were $-0.92 \%$ and $2.77 \%$, respectively, while $0.67 \%$ and $1.68 \%$ for cooling as shown in Figure 4.8 . While, hourly MBE and CV (RMSE) for heating loads were $-0.93 \%$ and $4.46 \%$, respectively, while $0.67 \%$ and $3.04 \%$ for cooling. Figure 4.9 and Figure 4.10 shows comparison between hourly measured and simulated data for heating and cooling loads for different months, respectively. Figure 4.11 and Figure 4.12 provide a sample of a more focused comparison through two weeks of data in winter and summer, respectively.

The results are within the ASHRAE Guideline 14-2002 thresholds. Table 4.2 shows parameters used for optimization including initial and final values used for calibration, and the influence coefficient of each parameter on the simulation output. The sensitivity influence coefficient (IC) was calculated as follows in Equation 3.1 [209]:

$$
I C=\frac{\frac{\Delta O P}{O P_{\text {Base case }}}}{\frac{\Delta I P}{I P_{\text {Base case }}}}
$$

where, $\triangle O P$ and $\triangle I P$ are changes in output and input, respectively. While, $O P$ Base case and 
$O P$ Base case are the output and input values of the base case.

The air flow through windows has the most influence on performance because as the building contains operable windows for most of the teaching laboratories and some offices and opposite large sliding doors at the entrance, which causes significant airflow. On the other hand, the ground temperature recorded lower influence on the simulation output, as the basement contains mechanical and electrical rooms that are ventilated with separate air handling units than the rest of the building. Moreover, only a small portion $(\sim 10 \%)$ of the building surface area is below grade.
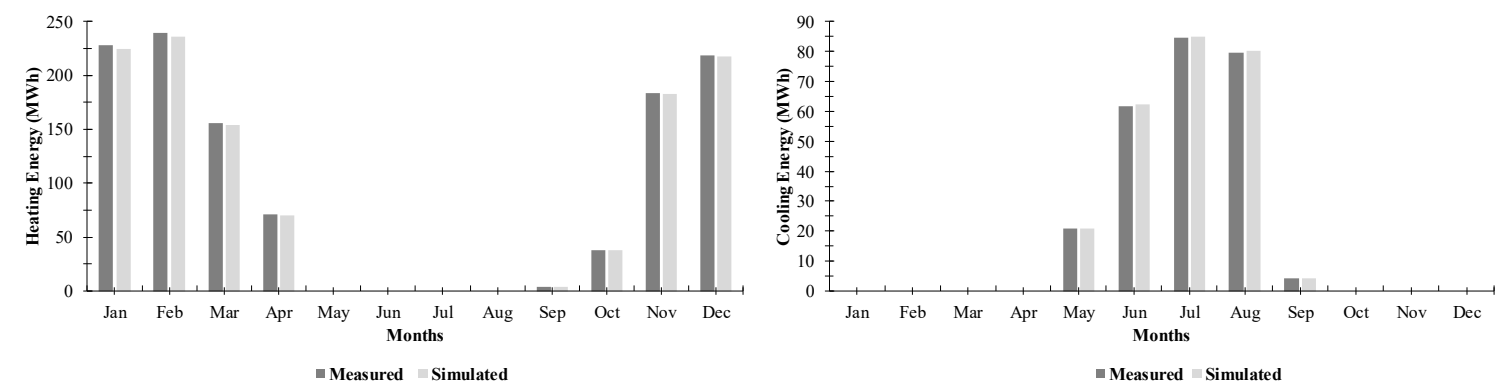

Figure 4.8: Comparison between monthly measured and simulated data for heating loads (left) and cooling loads (right)

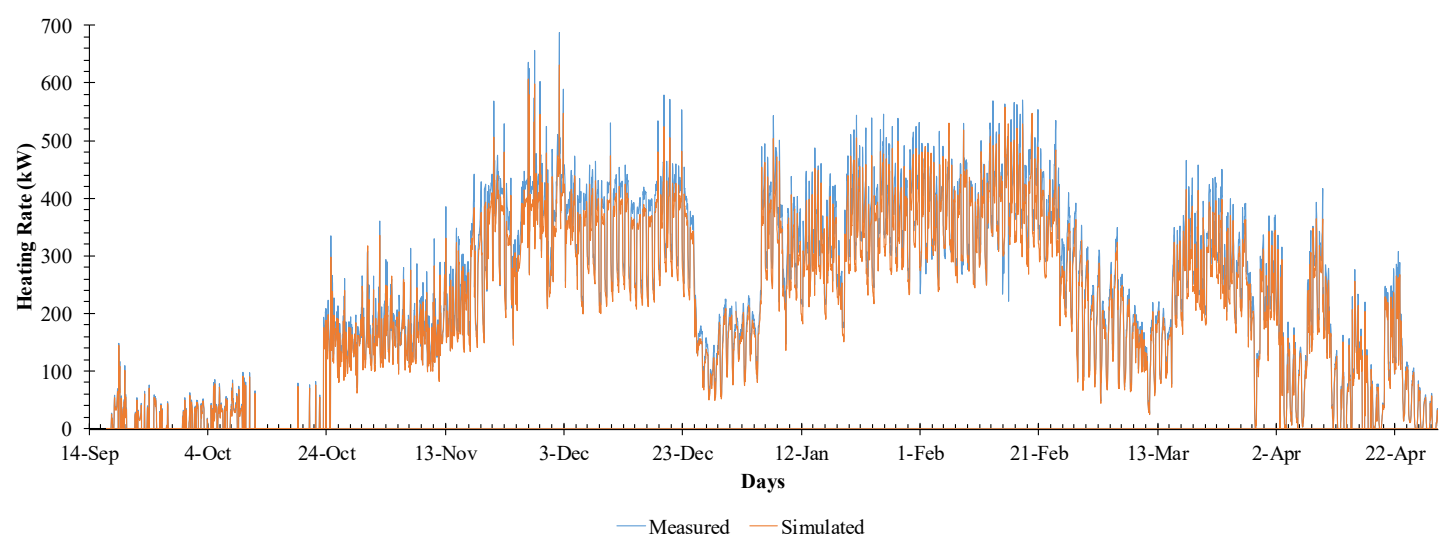

Figure 4.9: Comparison between hourly measured and simulated data for heating rate 


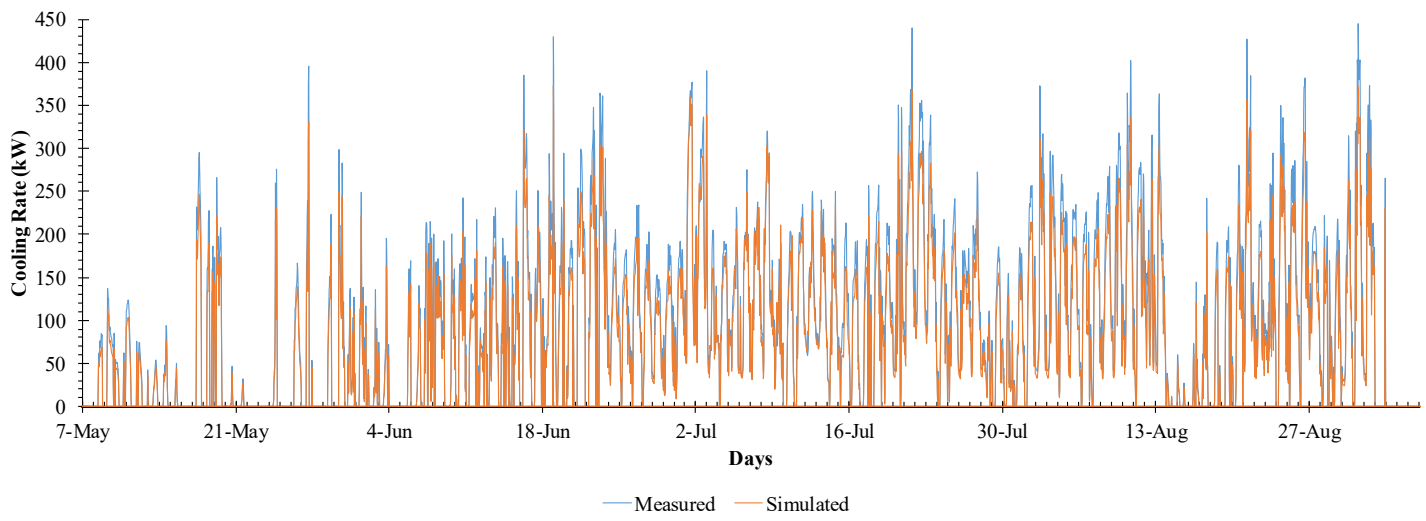

Figure 4.10: Comparison between hourly measured and simulated data for cooling rate

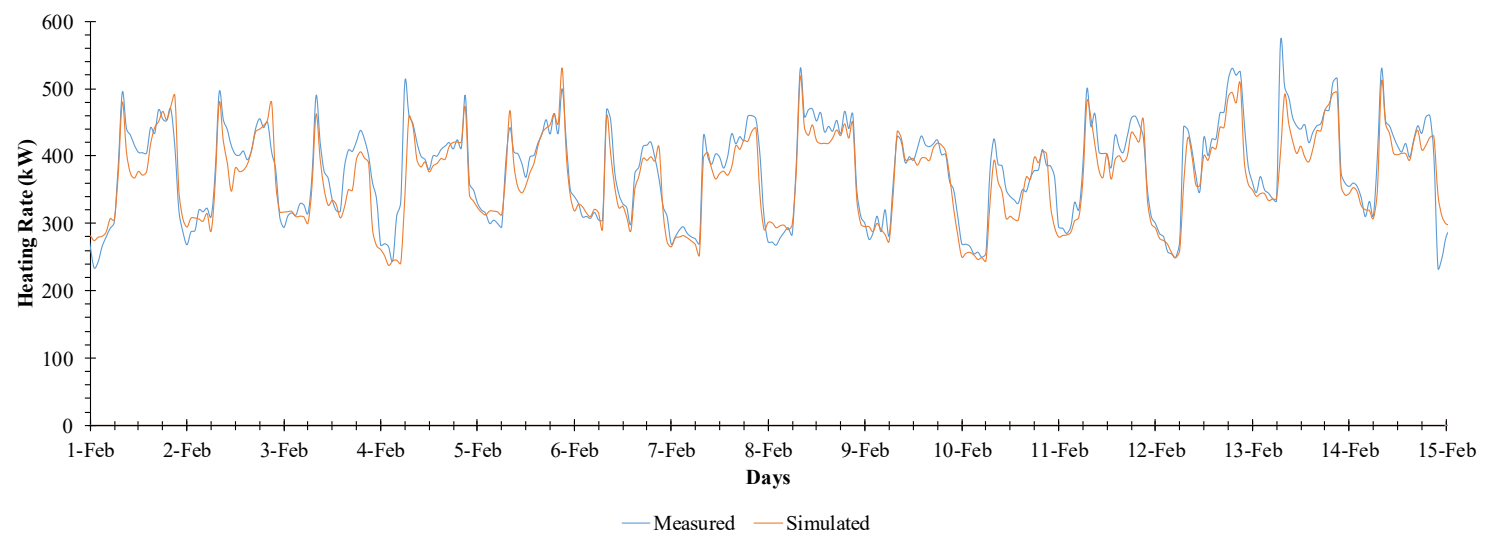

Figure 4.11: Comparison between hourly measured and simulated data during two weeks in winter

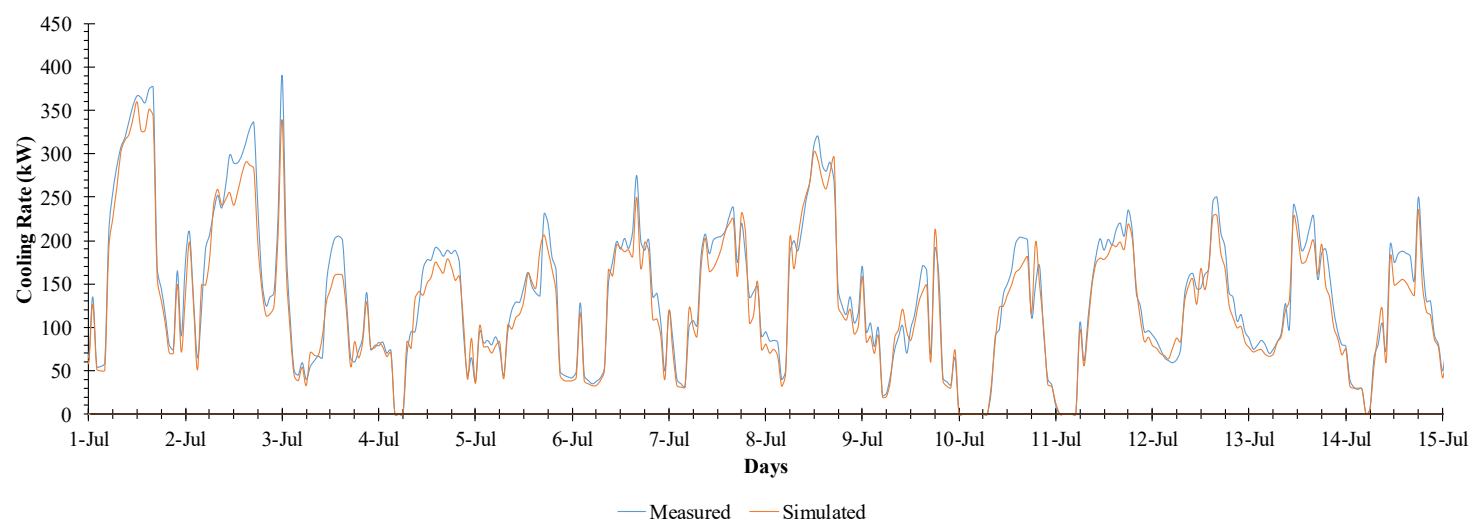

Figure 4.12: Comparison between hourly measured and simulated data during two weeks in summer 
Table 4.2: Parameters used for optimization

\begin{tabular}{|c|c|c|c|c|c|c|c|c|}
\hline \multirow{2}{*}{$\begin{array}{l}\text { Identifiable } \\
\text { Parameters }\end{array}$} & \multirow[t]{2}{*}{ Units } & \multirow{2}{*}{$\begin{array}{l}\text { Minimum } \\
\text { values }\end{array}$} & \multirow{2}{*}{$\begin{array}{l}\text { Maximum } \\
\text { values }\end{array}$} & \multirow{2}{*}{$\begin{array}{l}\text { Step } \\
\text { size }\end{array}$} & \multirow{2}{*}{$\begin{array}{c}\text { Initial } \\
\text { model } \\
\text { parameters }\end{array}$} & \multicolumn{2}{|c|}{$\begin{array}{l}\text { Final calibration } \\
\text { values (by season) }\end{array}$} & \multirow{2}{*}{$\begin{array}{c}I C(b y \\
\text { season) }\end{array}$} \\
\hline & & & & & & Heating & Cooling & \\
\hline $\begin{array}{l}\text { Air flow through } \\
\text { windows } \\
\text { (effective area of } \\
\text { the total operable } \\
\text { window area) }\end{array}$ & Fraction & 0 & 1 & 0.05 & 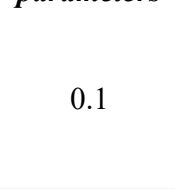 & 0.15 & 0.3 & $\begin{array}{c}(0.85 \\
\text { heating }) \\
(0.78 \\
\text { cooling) }\end{array}$ \\
\hline $\begin{array}{l}\text { Infiltration rate } \\
\text { (perimeter zones) }\end{array}$ & $\begin{array}{l}\mathrm{ACH} \\
@ 50 \mathrm{~Pa}\end{array}$ & 0.1 & 0.8 & 0.05 & 0.3 & 0.2 & 0.2 & $\begin{array}{c}(0.76 \\
\text { heating })(0.7 \\
\text { cooling })\end{array}$ \\
\hline $\begin{array}{l}\text { Insulation } \\
\text { conductivity }\end{array}$ & $(\mathrm{W} / \mathrm{m} . \mathrm{K})$ & 0.025 & 0.2 & 0.002 & 0.03 & \multicolumn{2}{|c|}{0.035} & $\begin{array}{c}(0.21 \\
\text { heating }) \\
(0.15 \\
\text { cooling })\end{array}$ \\
\hline $\begin{array}{l}\text { Number of } \\
\text { occupants in } \\
\text { classrooms }\end{array}$ & Fraction & 0.1 & 1 & 0.05 & 0.4 & 0.8 & 0.6 & $\begin{array}{c}(0.13 \\
\text { heating })(0.2 \\
\text { cooling })\end{array}$ \\
\hline $\begin{array}{l}\text { Ground } \\
\text { reflectivity } \\
\text { (albedo) with } \\
\text { snow }\end{array}$ & Fraction & 0.5 & 0.7 & 0.05 & 0.5 & 0.65 & - & $\begin{array}{c}(-0.08 \\
\text { heating })\end{array}$ \\
\hline $\begin{array}{l}\text { Insulation specific } \\
\text { heat }\end{array}$ & (J/kg.K) & 940 & 1100 & 10 & 960 & 980 & & $\begin{array}{c}(0.073 \\
\text { heating }) \\
(-0.1 \\
\text { cooling })\end{array}$ \\
\hline Blind position & Fraction & 0 & 1 & 0.1 & 0.2 & 0.3 & 0.5 & $\begin{array}{c}(0.057 \\
\text { heating) } \\
(-0.036 \\
\text { (cooling) }\end{array}$ \\
\hline $\begin{array}{l}\text { Ground } \\
\text { temperature (Jan, } \\
\text { Feb, Mar, Apr, } \\
\text { May, Jun, Jul, } \\
\text { Aug, Sep, Oct, } \\
\text { Nov, Dec) }\end{array}$ & ${ }^{\circ} \mathrm{C}$ & $\begin{array}{c}(-2.5,-5.5 \\
-5,-3.5 \\
3.5,8.5 \\
13.5,16 \\
15.5,12.5 \\
7,1.5)\end{array}$ & $\begin{array}{c}(1.5,1,2 \\
3,10,15 \\
20,23,22 \\
19,13,7)\end{array}$ & 0.1 & $\begin{array}{c}(-3,-5,-4.5 \\
-3,4,9,14 \\
16.5,16,13 \\
7.5,2)\end{array}$ & $\begin{array}{r}(-1.05,- \\
0.95,5.1 \\
17.3,16 \\
8.75\end{array}$ & $\begin{array}{l}5,-2.8,- \\
0.6,15.1 \\
5,13.75 \\
.45)\end{array}$ & $\begin{array}{c}(-0.033 \\
\text { heating }) \\
(0.023 \\
\text { cooling })\end{array}$ \\
\hline $\begin{array}{l}\text { Infiltration rate } \\
\text { (core zones) }\end{array}$ & $\begin{array}{l}\mathrm{ACH} \\
@ 50 \mathrm{~Pa}\end{array}$ & 0.01 & 0.1 & 0.01 & 0.05 & 0.03 & 0.03 & $\begin{array}{l}(0.012 \\
\text { heating }) \\
(0.02 \\
\text { cooling })\end{array}$ \\
\hline $\begin{array}{l}\text { Ground } \\
\text { reflectivity } \\
\text { (albedo) without } \\
\text { snow }\end{array}$ & Fraction & 0.5 & 0.7 & 0.02 & 0.2 & - & 0.26 & $\begin{array}{l}(0.017 \\
\text { cooling })\end{array}$ \\
\hline
\end{tabular}

\subsection{Data Visualization}

This section includes samples of data visualization of energy flow and the associated cost using Sankey diagrams on the building level for summer and winter periods. The Sankey diagrams shown in this section focused on historical data for four months in winter (January-April 2015) and summer (May-September 2015). Sankey diagrams should be read based on the direction of the flow (i.e. from left to right). All 
inputs come from the left side, while outputs leave rightward. For instance, during winter, heat is actively added to the building. While during summer, heat is actively extracted from the building. Furthermore, the proposed Sankey diagrams aimed at comparing the actual performance of different components (i.e. sensed and metered components such as lights, equipment, AHU-fans, pumps, steam, chillers, and cooling tower fans) to the baseline energy consumption for the same selected period that was provided by the Facilities Management and Planning (FMP) on campus. The baseline energy consumption is calculated based on previous year's bills. Green, yellow, and red colors were used to indicate the performance levels that are higher, equal to, and lower than baseline energy consumption, respectively.

Sankey diagrams were rendered in a browser using Scalable Vector Graphics (SVG). The layout was derived from D3's Sankey layout code developed by Google Developers that provided more flexibility in organizing nodes, colors, and font sizes [183]. In order to create nodes with the connection links, a set of rows containing data for source, target, and magnitude for each flow was created using comma-separated values (CSV) format.

\subsubsection{Energy Consumption}

The purpose of this diagram is to show the energy flow from the source (i.e. the secondary energy sources/ on-site energy) and its distribution to different components on the building level. The amount of energy gains and losses were obtained from measured and modeled data. All forms of energy (i.e. electricity and steam) in this diagram were converted to one common energy unit (MWh). Building-level energy flow for heating and cooling seasons are shown in Figure 4.13 and Figure 4.14, respectively. 


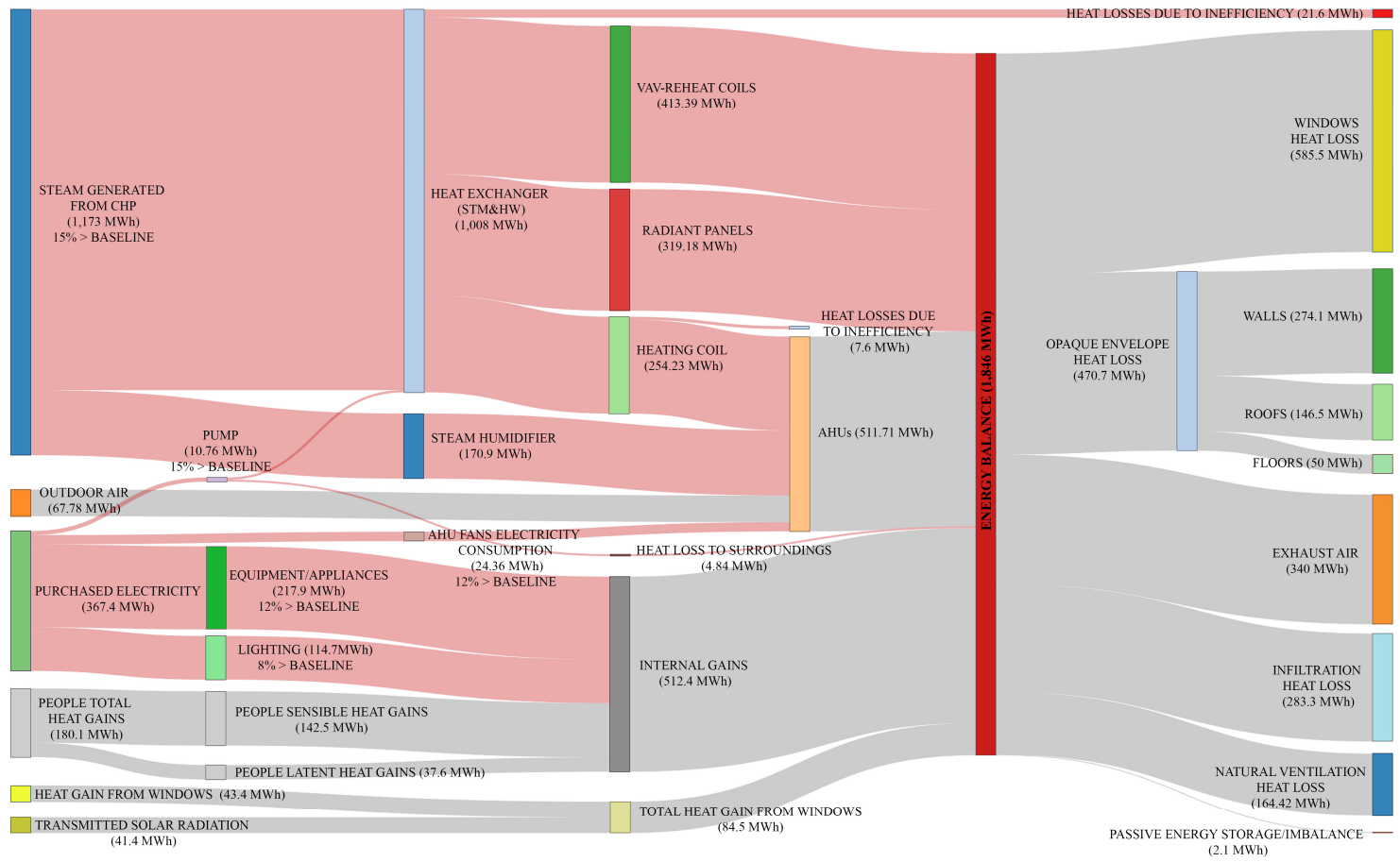

Figure 4.13: Sankey diagram showing building-level energy flow for the winter season, energy flows (for sensed and metered components) colored in red color indicates that the actual energy consumption is higher than the baseline

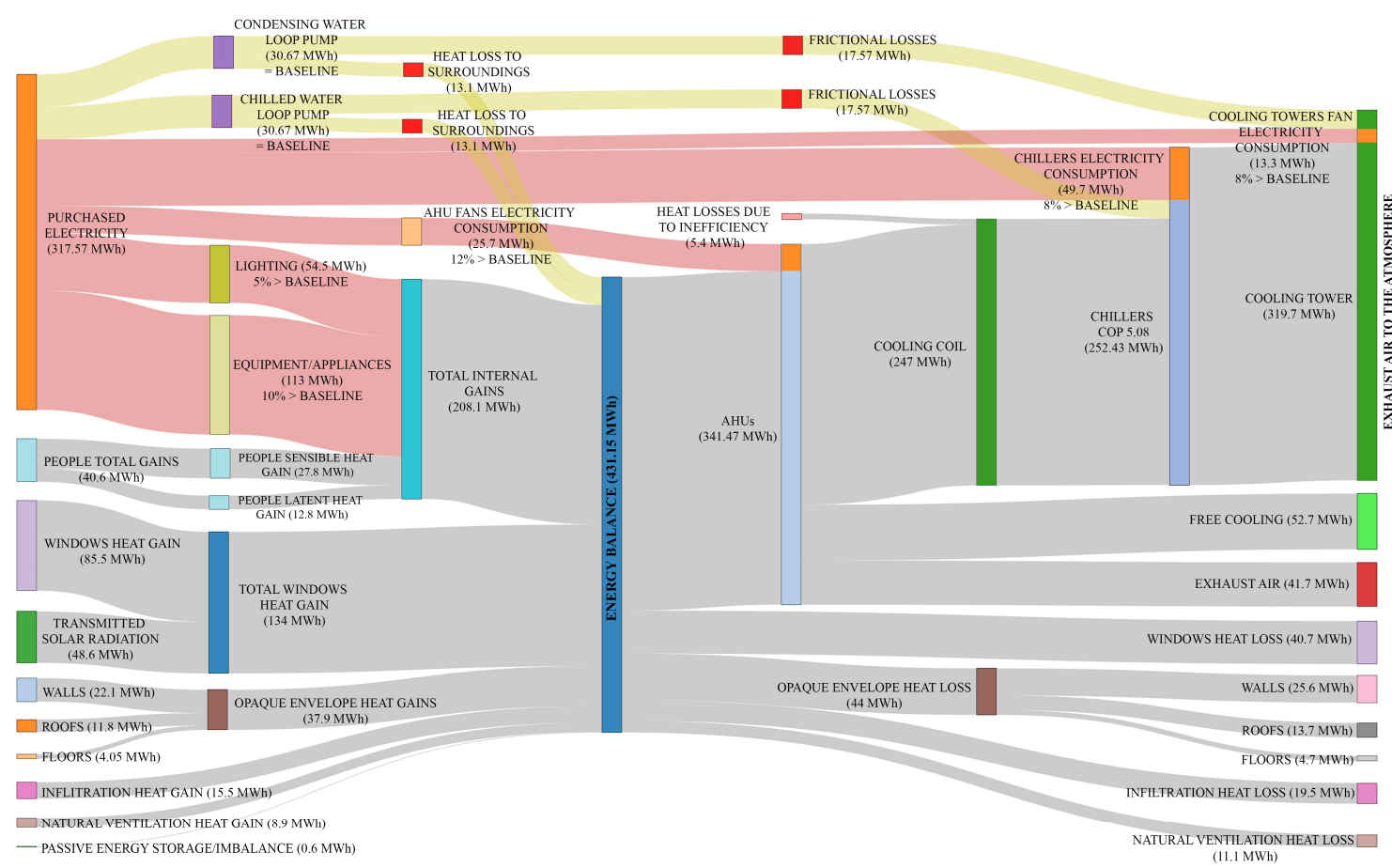

Figure 4.14: Sankey diagram showing building-level energy flow for summer season, energy flows (for sensed and metered components) colored in red color or yellow indicates that the actual energy consumption is higher than or equal to the baseline, respectively 


\subsubsection{Energy Cost}

The energy cost is allocated to the grid electricity and steam generated from central heating plant. The cost of the steam was determined based on the amount of natural gas and electricity consumption by the central heating plant [197]. Moreover, the equivalent indirect cost of passive heat gains or losses was also considered by using a proportional weighting to map these costs to real utility costs. Furthermore, electrical to thermal cost ratio was also considered during heating season to determine the value of heating cost that is offset by lighting, equipment/appliance, air handling unit fans, and pumps. On the other hand, energy cost was calculated based on the coefficient of performance (COP) of the cooling system during summer days to be able to determine the value of adverse and useful repercussions on cooling cost. The left-hand side of the diagram shows the total purchased electricity by the chillers and cooling tower fans and the indirect cost of passive heat losses to remove heat from building. While, on the right-hand side of the diagram, it shows the cost to remove the corresponding heat from equipment, lighting, pumps, air handling unit fans, and indirect cost of passive heat gains. For example, the direct electricity cost to operate the lights in the summer was $\$ 5,994$, but the cost to remove the corresponding heat was reduced by the effective COP of the cooling system and, thus, only amounted to $\$ 1,241$. In contrast to the Sankey diagrams representing energy, there is not necessarily a conservation of cost across a component. Thus, direct translation from energy to cost for the energy cost Sankey diagram is a non-trivial exercise. This study excluded the cost of water consumption by sinks, drinking fountains, and laboratories. Figure 4.15 and Figure 4.16 show energy cost during heating and cooling seasons, respectively. 


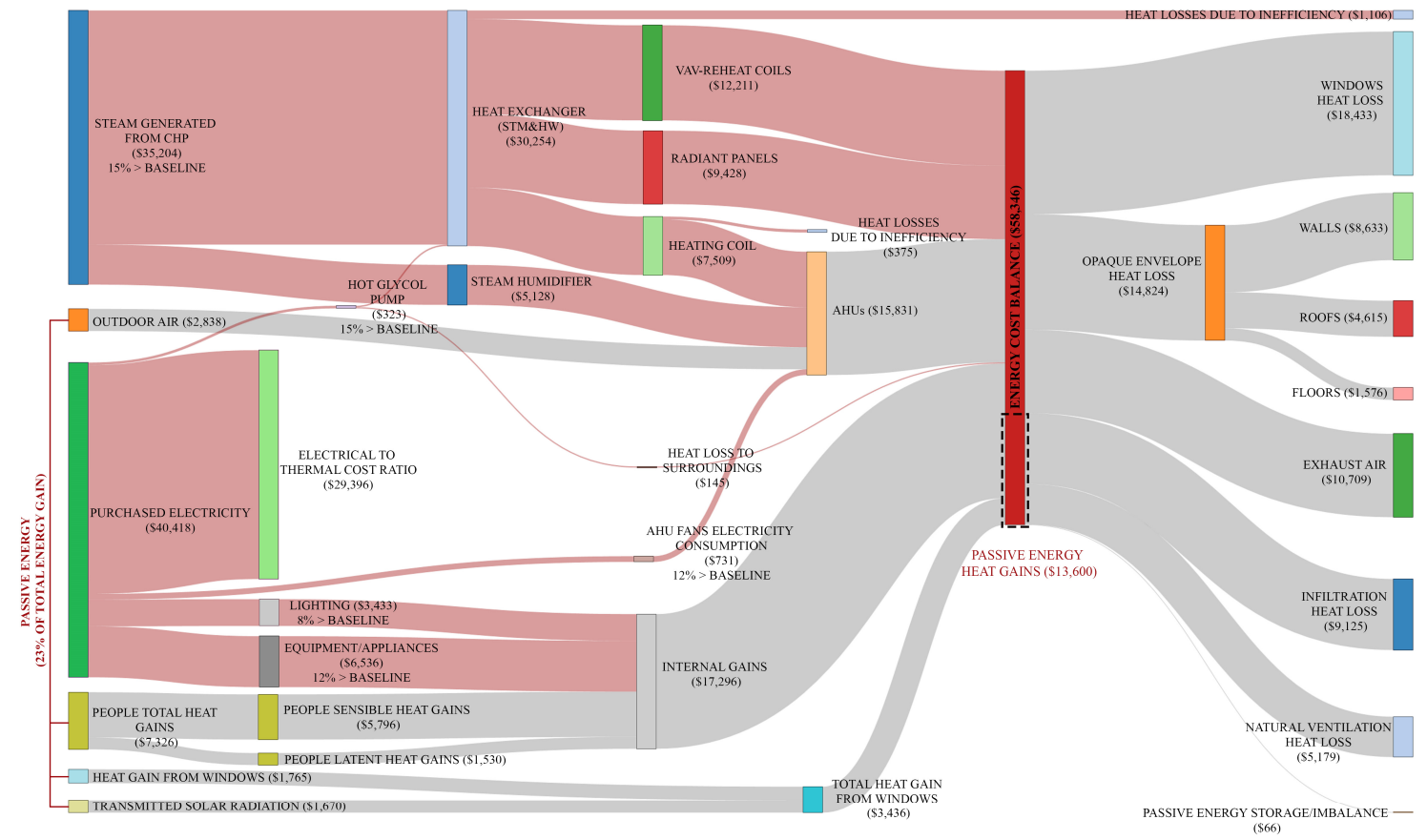

Figure 4.15: Sankey diagram showing energy cost during winter season, energy costs (for sensed and metered components) colored in red color indicates that the actual energy consumption is higher than the baseline

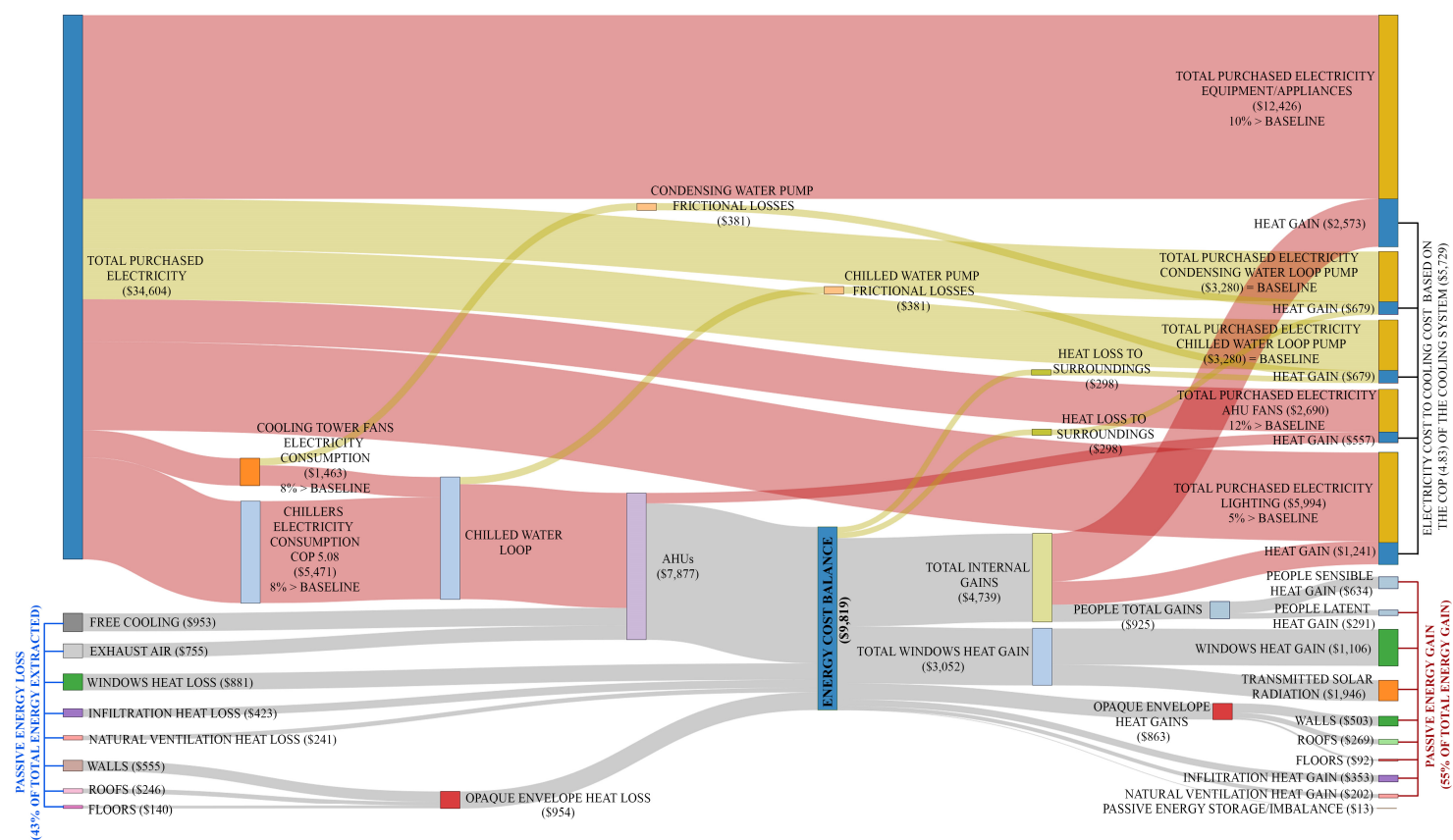

Figure 4.16: Sankey diagram showing energy cost during summer season, energy costs (for sensed and metered components) colored in red color or yellow indicates that the actual energy consumption is higher than or equal to the baseline, respectively 


\subsection{Results and Discussion}

This study focused on sub-hourly data obtained from a calibrated building performance simulation (BPS) model of the Canal building during winter and summer seasons. Figure 4.13 shows the energy flows on the building level during the heating season. The heating demand of the building was relatively high $\left(\sim 146 \mathrm{kWh} / \mathrm{m}^{2}\right)$ compared to the average commercial buildings energy use intensity in Canada $\left(\sim 105 \mathrm{kWh} / \mathrm{m}^{2}\right)$ [5]. It was also found that the hot glycol water pump energy consumption was higher than the baseline by approximately 15 percent. This is partially due to the fact that the set point temperature is set to $22^{\circ} \mathrm{C}$, regardless of occupancy. Moreover, some spaces had their windows predominantly left open in the winter. And importantly, opposite pairs of large sliding doors at the entrance causes significant airflow through the building lobby. Approximately 64 percent of heat was added from the steam delivered from the CHP during the winter, while the rest was obtained through heat gains from people, lighting and equipment, as shown in Figure 4.13. The vast majority of heating is delivered to the spaces through radiant panels and VAV reheat coils, with the remainder delivered to the air handling unit (AHU) as steam or sensible heat. The amount of energy added by outdoor air was approximately 13 percent of AHU energy. The lighting and equipment/appliances energy consumption was found to be higher than the baseline by 8 and 12 percent, respectively. The lighting energy consumption was high during unoccupied hours for security purposes; they were left on for hallways, lobby, and washrooms. Some of the teaching labs and some occupants used to leave their PCs switched-on during unoccupied hours, which lead to a high energy consumption for equipment and appliances. In conclusion, it was found that occupant behavior and operational schedules have a 
significant impact on energy consumption. The highest source of heat loss in the heating period was from the windows due to the high temperature difference between outdoor (ranges from -26 to $-5^{\circ} \mathrm{C}$ ) and indoor (ranges from 20 to $22^{\circ} \mathrm{C}$ ), large window area, and relatively high $U$-value.

During cooling periods, approximately 73 percent of the heat extracted from the building was achieved using the cooling coils in the air handling unit (AHU), as shown in Figure 4.14. The chilled water (water-propylene glycol) for the case study, extracts heat from the water in the cooling coils and transfers it to the condensing water by means of vapor compression cycle (chillers). The cooling tower extracts heat from the condensing water to the environment. Moreover, some of the heat is exhausted to the environment from air handling unit (AHU) exhaust dampers. The building also relies on free cooling (i.e., by increasing the supply of cooler outdoor air instead of mechanical cooling) when the outdoor temperature is below $22^{\circ} \mathrm{C}$. It was also found that some heat loss occurred through natural ventilation and exfiltration as the outdoor temperature ranged from 15 to $30^{\circ} \mathrm{C}$, while the indoor temperature ranged from 22 to $24^{\circ} \mathrm{C}$. Approximately $31,26,13,9,9,4,2$, and 6 percent of the heat was added by windows, equipment/appliances, lighting, people, opaque envelope, infiltration, natural ventilation, and heat loss from pumps, respectively. It was again observed that the lighting and equipment energy consumption was higher than the baseline by five and 10 percent, respectively, as discussed above. The chillers energy consumption was found to be higher than the baseline by approximately 8 percent. This was due to the high internal gains from occupants, lighting, equipment, and heat gain from building envelope. Furthermore, while the coefficient of performance (COP) of the chiller is approximately five, the one unit of electricity was required to remove 4.5 units of heat 
from the building. The pumps (i.e. condensing and chilled water) energy consumption were found to be the same as baseline. While, the cooling tower fans energy consumption were found to be higher than the baseline by approximately 8 percent.

On the energy cost side, Figure 4.15 shows total purchased energy was found to be of $\$ 75,622$ during the heating period. Approximately 53 percent of the energy cost was allocated to electricity and 47 percent to steam. On the hand, approximately $1173 \mathrm{MWh}$ ( $\sim 76$ percent) and $367 \mathrm{MWh}$ ( $\sim 24$ percent) of the total energy was allocated to thermal and electrical energy, respectively. The comparison between the cost and energy unit reveals that the thermal energy is much cheaper than electrical energy. The value of heating cost that is offset by equipment/appliance, lighting, air handling unit fans, and pumps was found to be approximately $11,6,1.2$, and 0.6 percent of the total heating cost, respectively. Moreover, around 23 percent of the total energy gain was from passive sources (such as people, outdoor air and windows).

In the cooling season, the total purchased electricity cost was approximately $\$ 34,604$ allocated to equipment, lighting, air handling unit (AHU) fans, chillers, pumps, and the cooling tower fans, as shown in Figure 4.16. Approximately 55 percent of the total cooling cost was from passive sources (such as people, windows, opaque envelope components, infiltration and natural ventilation). While, 45 percent of total cooling cost was from electricity consumption by equipment/appliance, lighting, pumps, and air handling unit fans. On the other hand, 43 percent of the cooling cost was offset by passive means from the building (such as windows, opaque envelope, exhaust air, free cooling, exfiltration and natural ventilation). 
The Sankey diagrams developed in this study helped to reveal inefficiencies of different building components and systems. For instance, the windows caused the highest heat loss during winter period. During the cooling period, the highest source of heat gain was also the windows. Windows are ruled by solar heat gain coefficient SGHC and Uvalue. A lower U-value would reduce heat loss in the winter and heat gain during summer. On the other hand, lower SGHC would reduce heat gains leading to more heating in winter and less cooling in summer. Thus, if these two parameters are selected carefully, the overall result would be a reduction in both cooling and heating. It was also noticed that the lighting resulted in high internal gains in both seasons. Thus, higher efficiency light bulbs/fixtures could be installed to reduce the amount of heat gain during summer. This will result in reducing the amount of electrical energy consumption by the cooling system. However, by reducing heat gain from lighting, this will lead to an increase in the heating demand during winter period. This will result in increasing the amount of steam consumption. Furthermore, the electrical energy required to remove heat from the building in summer is based on the coefficient of performance (COP) of the cooling system. Thus, by installing chillers with higher COP and cooling towers with higher efficiency, this will result in having higher COP of the cooling system and therefore less cooling cost.

\subsection{Survey}

An online survey was used to assess usability and effectiveness of visualizing energy flow and the associated cost using Sankey diagrams. The survey used the same Sankey diagrams as shown in this study. The survey questions are mentioned in Appendix C. 2. The survey was applied to a sample user group (42 participants). The characteristics of the sample group varied according to profession and years of experience. The sample 
group consisted of $31 \%$ energy managers, $29 \%$ design engineers, $14 \%$ researchers/lecturers, $7 \%$ architects, $7 \%$ building energy analyst, 5\% building envelope consultants, $5 \%$ other building-related technician, and $2 \%$ property managers. The majority of the users (43\%) had 5-10 years of experience, while, $26 \%, 17 \%$, and $14 \%$ of the users had $0-5,10-20$, and more than 20 years of experience, respectively.

The survey consisted of four sections. Section 1 asked participants about the effectiveness of using Sankey diagrams in visualizing building-level energy flows and to understand the interrelated variables that affect building performance (such as the effect of internal gains to the heating and cooling loads). Figure 4.17 shows participants' overall impression of the Sankey diagrams.

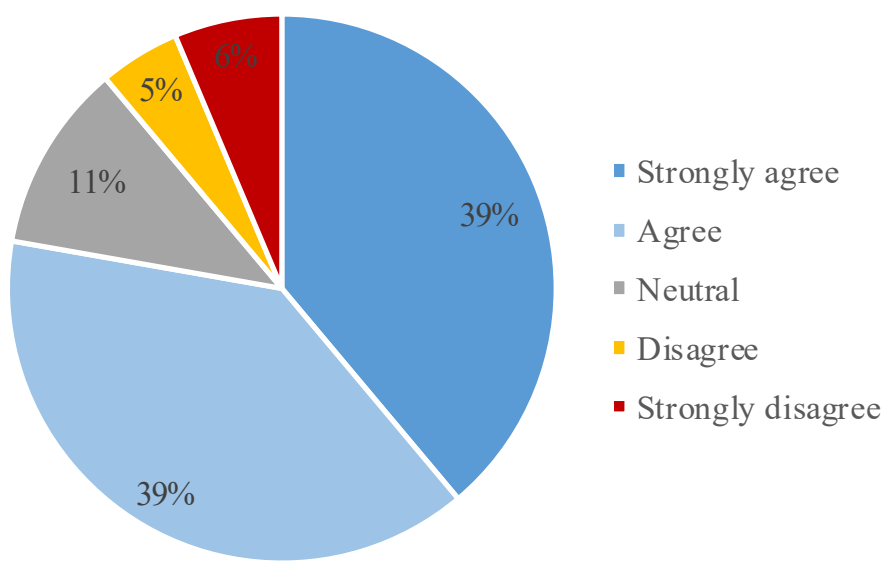

Figure 4.17: Survey results on the effectiveness of using Sankey diagrams to visualize building-level energy flows

In the following sections ( $2 \& 3)$, the users were asked to perform tasks that required interpreting the Sankey diagrams. Section 2 aimed at interpreting energy flows using Sankey diagrams for cooling and heating seasons. In this section, the users were asked to determine the path responsible for the most cooling and heating energy. The survey results are shown in Figure 4.18. The results showed that ( $78 \%)$ of the users selected the correct 
answer as shown in building-level energy flow Sankey diagrams.

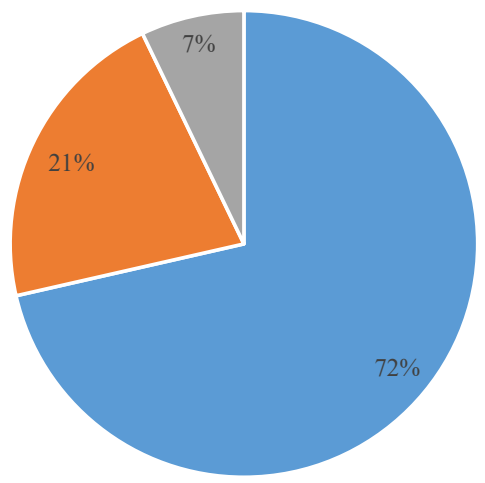

- Windows

- Equipment/appliances

- I don't understand

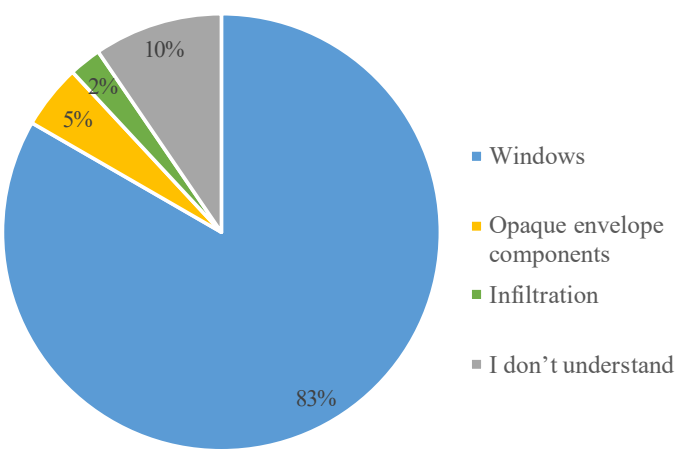

Figure 4.18: Survey results on determining the path responsible for the most cooling (left) and heating (right) energy

Section 3 focused on evaluating Sankey diagrams in visualizing energy cost. Most of the users $(\sim 80 \%)$ found that Sankey diagrams are useful because they show the breakdown of individual flow components. Moreover, it helped to visualize the costs in relation to one another. In this survey section, the users were asked to determine the ratio of passive to active energy cost during heating and cooling seasons as shown in Figure 4.19. In addition, they were asked to determine which building component would be most effective at reducing heating and cooling cost as shown in Figure 4.20. The results showed that $\sim 75 \%$ of the users selected the correct answer as shown in energy cost Sankey diagrams.

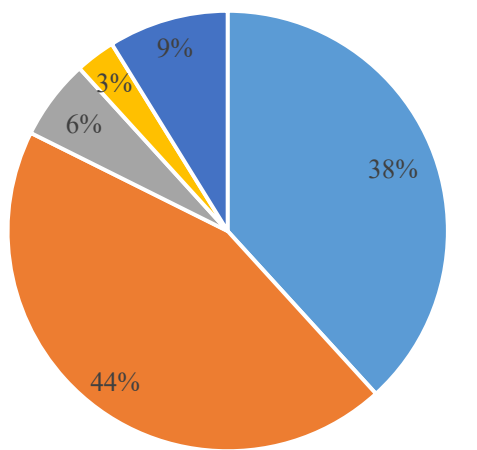

$$
\begin{aligned}
& =0-10 \% \\
& =10-20 \% \\
& =20-30 \% \\
& =30-40 \% \\
& =\text { more than } 50 \%
\end{aligned}
$$

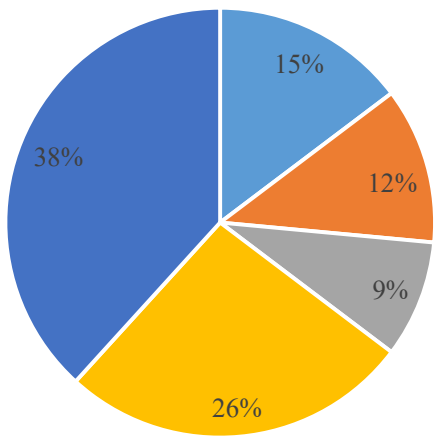

- $0-10 \%$

- $10-20 \%$

- $20-30 \%$

$=30-40 \%$

- more than $50 \%$

Figure 4.19: Survey results on determining the ratio of passive to active energy cost during heating (left) and cooling (right) energy 


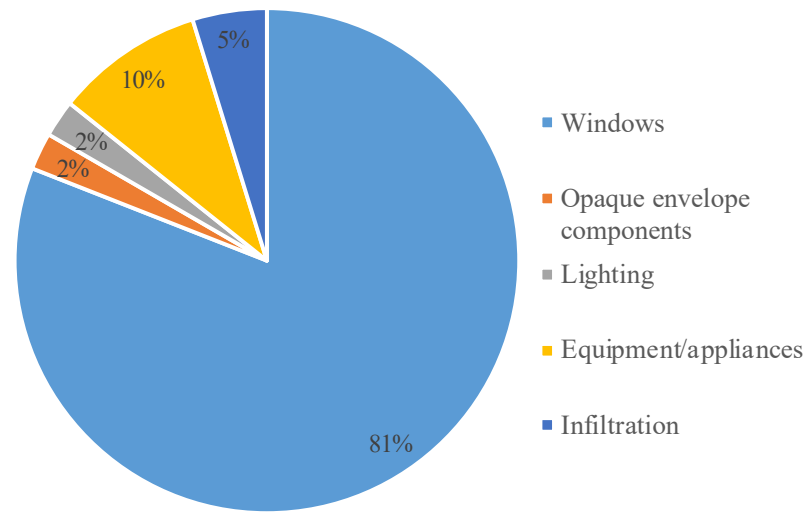

Figure 4.20: Survey results on determining the building component that would be most effective at reducing combined cost of heating and cooling

The findings of the survey from Sections 2 and 3 revealed that the remaining participants got the wrong answer as the difference between the correct answer and some energy paths/or costs were small. Moreover, some other participants were confused between similar jargon (such as passive energy costs, active energy cost, passive energy loss, passive energy gain, etc.). This could be resolved by providing more explanation to these terminologies.

Section 4 addressed the usefulness of Sankey diagrams to facilitate decisionmaking, as shown in Figure 4.21. The users were also asked on how likely they would be use Sankey diagrams to visualize energy flow and cost on the building-level, as shown in Figure 4.22. 


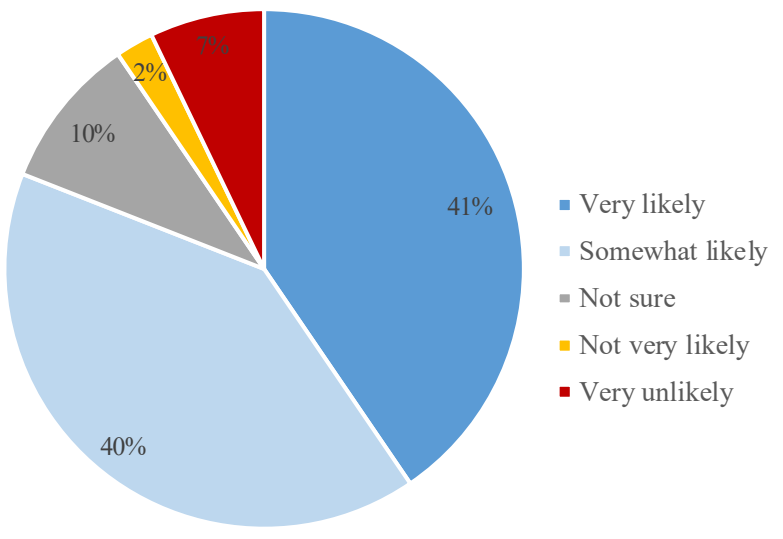

Figure 4.21: Survey results on evaluating the usefulness of Sankey diagrams to facilitate decision-making

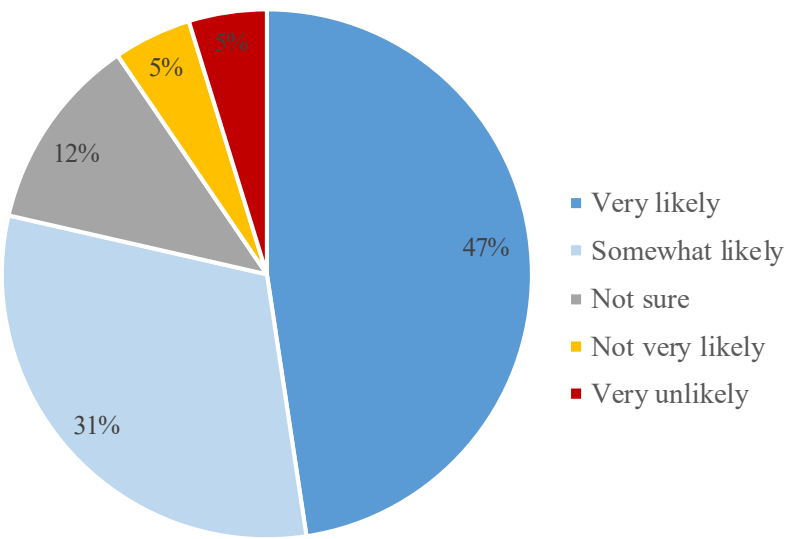

Figure 4.22: Survey results showing how likely the users would use Sankey diagrams to visualize energy flow and cost on the building-level

Some participants provided suggestions for improving the Sankey diagrams that were shown. It was suggested that a detailed explanation on how to use Sankey diagrams through a short video be provided. Moreover, some participants suggested making the Sankey diagrams more interactive. This could allow users to interrogate the data across custom timeframes (e.g. for the worst cooling month) or expand more general flows into more specific ones (e.g. digging into "window gains/losses" to specific orientations or even specific windows). 


\subsection{Summary and Future Work}

The aim of the study was to develop, demonstrate, and test a method to create Sankey diagrams that show whole-building energy flows and costs based on measured data and a calibrated energy model. The main objective of this study was to provide a comprehensive energy-use assessment tool that could facilitate the decision making by building owners, operators, and other stakeholders. The main contribution of this study is to provide an integrated framework for converting sparse measured data and a BPS model into a novel energy and cost visualization method that could be generalized to other existing modern buildings. Key components of framework include: 1) develop BIM model and convert the BIM-based model to a building performance model, 2) perform model calibration, 3) convert model data to reliable energy flows and cost and produce Sankey diagrams. The proposed method was applied to a large Canadian university building. The study was followed by a survey of building energy professionals to assess Sankey diagrams' usability and effectiveness to communicate energy flows.

As revealed from the survey results highlighted in Section 4.7. The findings supported that the Sankey diagrams developed in this study helped in: 1) visualizing building-level energy flows and costs, 2) understanding the interrelated variables that affect building performance, 3) identifying system inefficiencies, 4) quantifying and understanding the impact of unmeasured energy flows, and 5) making operational problems more visible and quantifiable in order to identify opportunities for energy savings and facilitate decision making.

There are some limitations in the usability of the proposed Sankey diagrams. For instance, they primarily provide information on the efficiency of components but do not 
necessarily identify poor operations. Moreover, they show relative heat transfer (e.g., windows, infiltration). Another limitation in this study is that the calibration optimization may produce mathematically correct but physically meaningless results; therefore, the user needs to countercheck results before using them. Furthermore, the building performance simulation (BPS) model is based on many modeling assumptions and simplifications.

Future steps for this research initiative include:

- Developing tool(s) for automating the process that creates Sankey diagrams from sub-meters input file and model data output files;

- Creating Sankey diagrams that can represent retrofitting of some building components and their impact on the overall energy consumption;

- Visualization of energy flows on the zone/room level.

- Developing interactive Sankey diagrams by allowing the user to select the temporal and spatial resolution (discussed in Chapter 7). 


\section{Chapter 5: Development of Sankey Diagrams to Visualize Real HVAC Performance}

This chapter is in review at Energy and Buildings journal:

(Abdelalim A., O'Brien W. and Shi Z., "Development of Sankey Diagrams to Visualize

Real HVAC Performance," Energy and Buildings)

This chapter proposes a method to estimate and visualize energy and mass flows in addition to the corresponding cost through different components of heating, ventilation, and air-conditioning (HVAC) system using Sankey diagrams.

\subsection{Abstract}

One of the crucial elements to ensure the efficiency of building operations is to understand the dynamics of energy flows, control strategies, and occupant behavior in buildings. Currently, abundant sensors and sub-meters are installed in modern buildings to measure resource consumption at various levels of spatial and temporal resolution to help track and reduce energy use and greenhouse gas emissions. These sensors and sub-meters provide many data sources at the building level; however, these data are not necessarily in a readily comprehensible format. In addition, the current data availability and visualization tools have some limitations in identifying system inefficiencies and possible solutions. This paper proposes a method to estimate and visualize energy and mass flows in addition to the corresponding cost through different components of heating, ventilation, and airconditioning (HVAC) system using Sankey diagrams. The aim of this study is to facilitate accessibility of data and to identify system inefficiencies. A major contribution of the paper is to convert sparse sensor data into estimated energy flows for each major AHU and plant loops component. The proposed method is then applied to a large Canadian university 
building. This paper concludes by discussing the challenges in obtaining data from sensors and sub-meters. The proposed method helps in understanding the performance of the whole system - not just individual components. Moreover, it helps identify operational problems and quantify their impact.

\subsection{Introduction}

In Canada, space heating and cooling in modern commercial buildings account for 56 and five percent, respectively, of the total energy allocated for residential and commercial energy-use [210]. Oftentimes, modern commercial buildings are equipped with abundant sensors and sub-meters to measure resource consumption at various levels of spatial and temporal resolution. Moreover, building energy management systems (BMS) can be utilized in these buildings to monitor and optimize building systems during operation. Such features also help track and reduce energy use in buildings, and hence decrease the associated costs and greenhouse gas emissions. But despite the availability of many data sources at the building level, these data are not necessarily in a readily comprehensible and action-oriented format. The importance of this monitoring process is confirmed by Herzog and LaVine [211] and Claridge et al. [212]. In their work, they stated that significant energy savings between 15 and 40 percent can be achieved from monitoring and identifying system inefficiencies. Building management systems have improved over time in terms of monitoring and supervision capabilities [213]. This improvement has helped in making operational problems more visible and quantifiable. Furthermore, energy efficiency of heating, ventilation and air conditioning (HVAC) systems has become a central objective for energy policies [214, 215, 216], as highlighted by building energy regulations [217], building energy rating systems and certification schemes [218, 219], and 
the standardization of inspection, operation and maintenance (O\&M) plans of HVAC systems.

Recent research has focused on analyzing and evaluating HVAC systems energyuse. A study by Perez-Lombard et al. [220] proposed a wide-scope analysis of HVAC systems aiming to provide guidelines for easier energy analysis in the HVAC field. Their study focused on mapping energy flow from energy sources to final services and by the examination of the intermediate devices. The data used in Perez-Lombard study was based on average values of engineering ratios taken from real HVAC design. These data were gathered for the design and simulation of HVAC systems in office buildings. Sakulpipatsin et al. [221] analyzed buildings and HVAC systems through exergy analysis to track energy demand from the building side to the energy supply side. Fan et al. [222] used a model to evaluate the operation performance of HVAC system based on exergy analysis. The method developed by Fan et al. used TRNSYS to develop a detailed simulation model of an airport building, which was used to obtain the operation performance of HVAC system. However, the study focused on the performance of the main plant components and air handling unit (AHU) - not the performance of each component in the AHU. Moreover, Fan et al. claimed that in order to evaluate the operational performance and optimum potential of the existing HVAC system, object exergy efficiency and the exergy loss ratio are not suitable for analyzing the influence of the control strategy to operation efficiency. Franconi et al. [223] combined the first and second laws of thermodynamics to analyze and evaluate the operation performance of HVAC system. However, the calculations performed at the system level do not indicate plant end-use energy. Salsbury et al. [213] used simulation as a tool for performance validation and energy analysis of HVAC 
systems. The work done by Salsbury et al. described how to use the simulation as a performance validation tool. Moreover, Salsbury et al. claimed that it is required to develop ways of characterizing and visualizing HVAC system performance. Mendes et al. [224] presented a generic educational user-friendly environment using mathematical models for simulating HVAC systems. Those mathematical models have been integrated into the whole building hygro-thermal model. The above studies are limited to using simulations and predictions rather than measured data.

Alternatively, there are new diagnostic software tools utilized in modern commercial buildings to facilitate the detection and diagnosis of energy and other performance problems for HVAC systems [225]. The current metrics and visualization techniques available at different scales range from building system to building level has been described in the work of Gayeski et al. [226]. One of the powerful tools for HVAC troubleshooting is the trend analysis tool Austin [227]. Moreover, Meyers et al. [106] presented data visualization techniques of HVAC and lighting systems. The data visualization can help building operators to achieve substantial improvements in energy management and equipment maintenance. Meyers et al. also stated that in order to make the data visualization effective, it should be integrated to the operation and maintenance plan.

The typical data availability and visualization tools are useful for determining the consumption and performance patterns of different components on the building system level. However, these tools are not optimized for identifying operational issues and inefficiencies to operators. For instance, they generally do not directly provide unmeasured energy and mass flow rates. And instantaneous values do not lend themselves to 
determining problematic trends. Moreover, cause and effect (e.g., downstream impacts) are not very evident. Typically, these tools are limited in providing qualitative analysis (the impact of a single component performance within a system). For instance, they do not directly indicate how the supply fan energy consumption affects the state of the air stream inside the AHU, which affects the amount of heating and cooling demands to meet the setpoint temperature of the supply air. As an example, Figure 5.1 and Figure 5.2 show typical dashboards that are available to building operators.

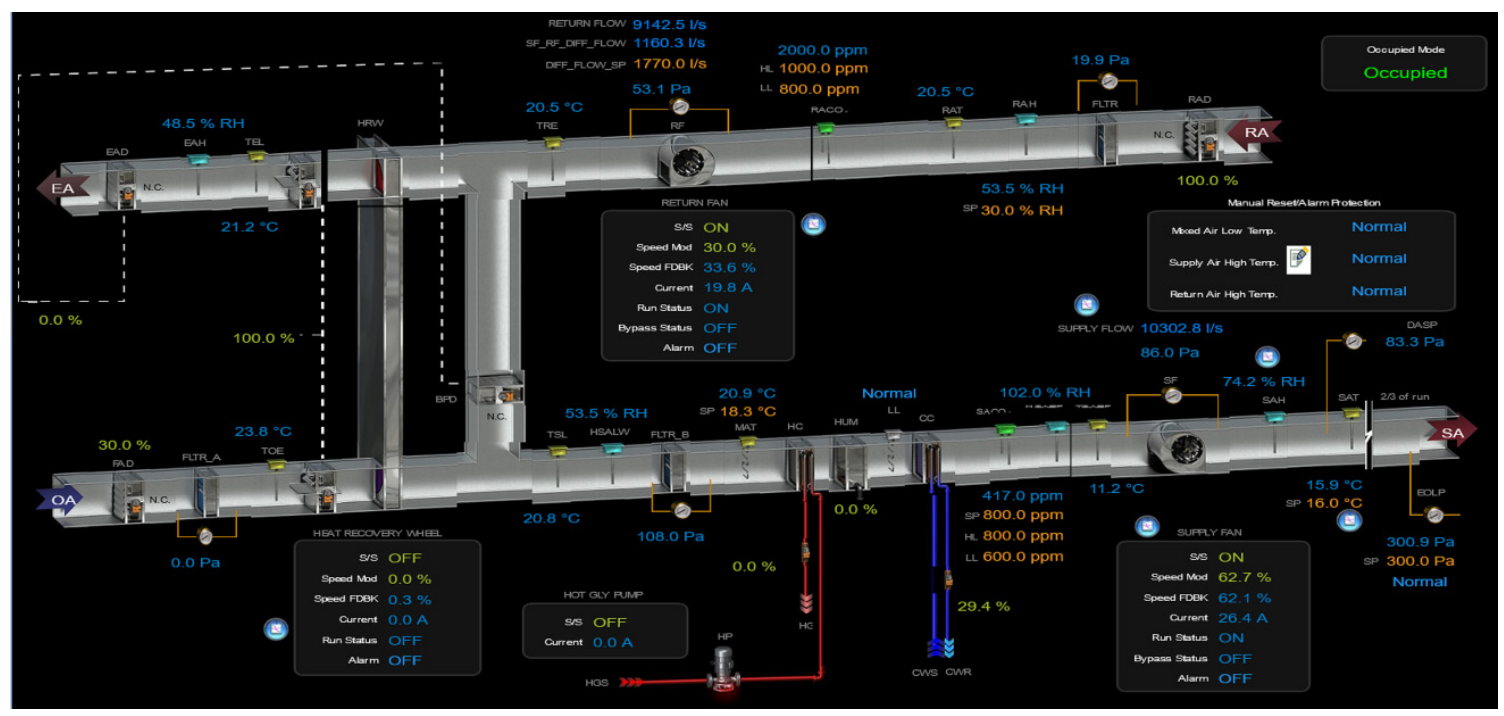

Figure 5.1: Energy dashboard for AHU implemented at Carleton University 


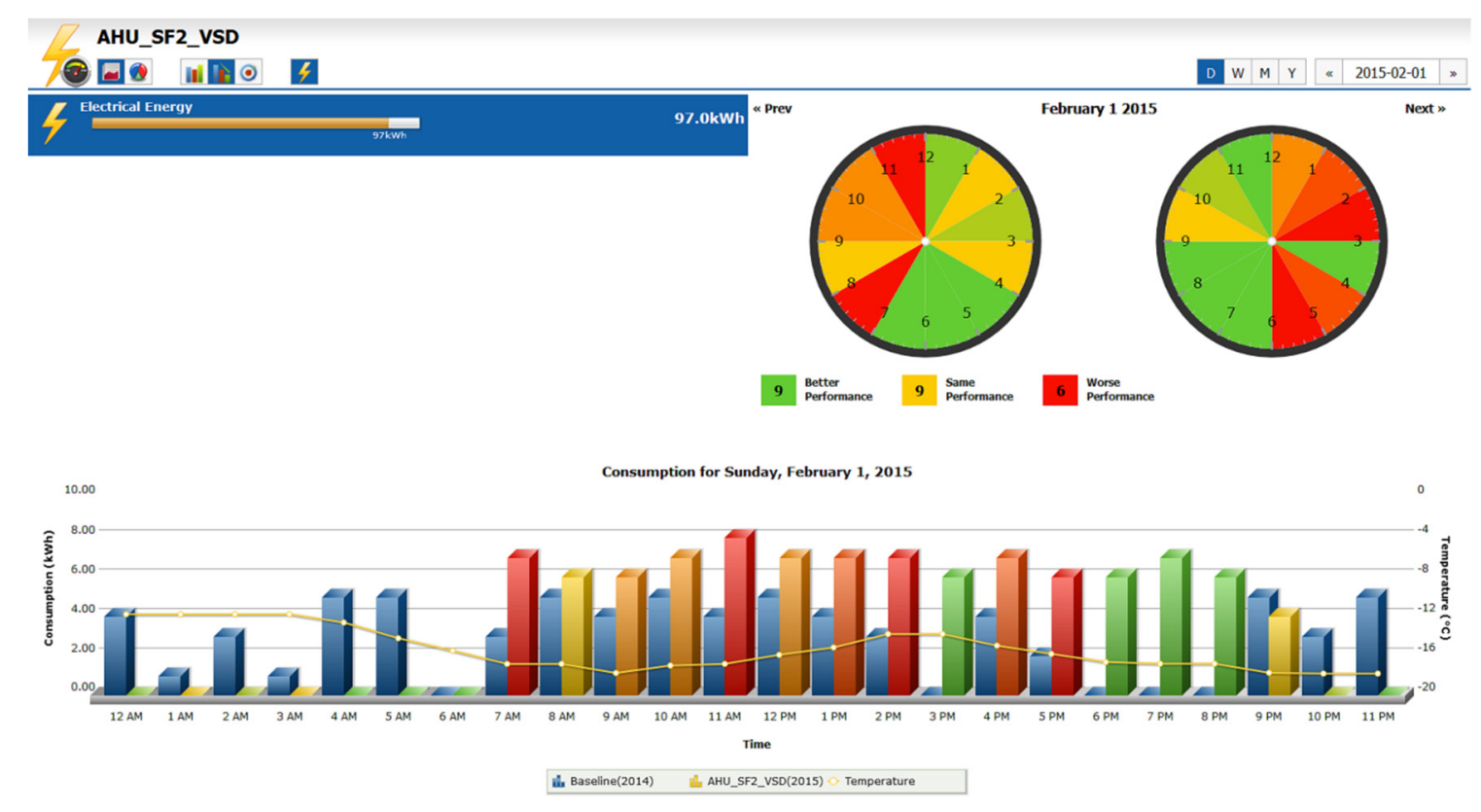

Figure 5.2: Supply fan hourly energy consumption

On the visualization side, the Sankey diagram is one of the visualization tools that is useful in energy management and performance improvement. In a companion paper by Abdelalim et al. [197], several methods were proposed to analyze energy and mass flows and the upstream environmental impacts on the campus level. Sankey diagrams and other graphical techniques were utilized to visualize campus metabolism. Another study by Abdelalim et al. [228] proposed a method to analyze and visualize energy flow and the associated cost by using historical data obtained from a BMS and a calibrated energy model to provide a comprehensive energy use assessment at the building level. Perez-Lombard et al. [220] utilized Sankey diagrams to visualize energy flows in a typical constant air volume systems installed in office buildings in Spain. There are various applications of using Sankey diagrams in visualizing energy flows of combined cooling, heat and power (CCHP), process heat from solar power, forced air and IR heating, and engine combined cycle power plant [229]. Sankey diagrams were also used to compare and contrast the 
performance of a domestic legacy gas ducted heating system with a contemporary, wellinstalled split-system heat pump heating system [230].

This study proposes a method to analyze and visualize energy, mass, and cost flows through different components of a typical AHU and plant loops of commercial buildings using Sankey diagrams. The aim of the proposed study is to demonstrate a new technique for visualizing real HVAC data to identify possible inefficiencies and inform operators on HVAC performance. As mentioned earlier, most of the current methods relied on simulations and predications to determine the performance of the HVAC system. This requires model calibration against measured data sets. Furthermore, the current methods/tools did not directly convert measured data obtained from sensors and sub-meters into energy flows to understand the actual performance of the HVAC system. This work contributes to the state-of-the-art by developing a methodology to use first principles to convert sparse HVAC data into energy, mass, and cost flows, and then to visualize these quantities using Sankey diagrams. The objective of this study is to make operational problems more visible and quantifiable. This could help to identify opportunities for energy savings and to facilitate the decision-making by building operators, campus planners, and other stakeholders. The proposed method was then applied to a case study: The Canal Building at Carleton University campus in Ottawa, Canada using historical data obtained from BMS. Sankey diagrams are utilized in this study to allow resource flows to be visualized within complex systems with interacting subsystems to uncover some issues. For instance, the effect of fan energy consumption on heating and cooling loads. This work also discusses the challenges in obtaining data from sensors and sub-meters. 


\subsection{Framework}

The framework of this study consists of two parts. The first part focused on converting historical data obtained from BMS into estimated energy and mass flows and the corresponding energy cost of a typical AHU and plant loops configurations of a commercial building which is discussed hereunder. The second part focused on utilizing Sankey diagrams to visualize energy and mass flows and the corresponding energy cost, which is discussed in Section 5.6. Figure 5.3 shows the process to analyze and visualize energy and mass flows and the corresponding cost using Sankey diagrams. The first part of the study focused on developing energy balances for AHU components (i.e. heat recovery wheels (HRW), fans, economizers, air filters, and cooling and heating coils) and plant loops (i.e. air loop, chilled water loop, refrigerant loop, condensing water loop, and heat rejection loop) as shown in Figure 5.4. The figure also shows the available sensors in the selected case study. Moreover, the study analyzed the feedback loops of different fluids (such as air, water, and steam loops) to determine the recirculated energy and mass flows as inputs back into the system.

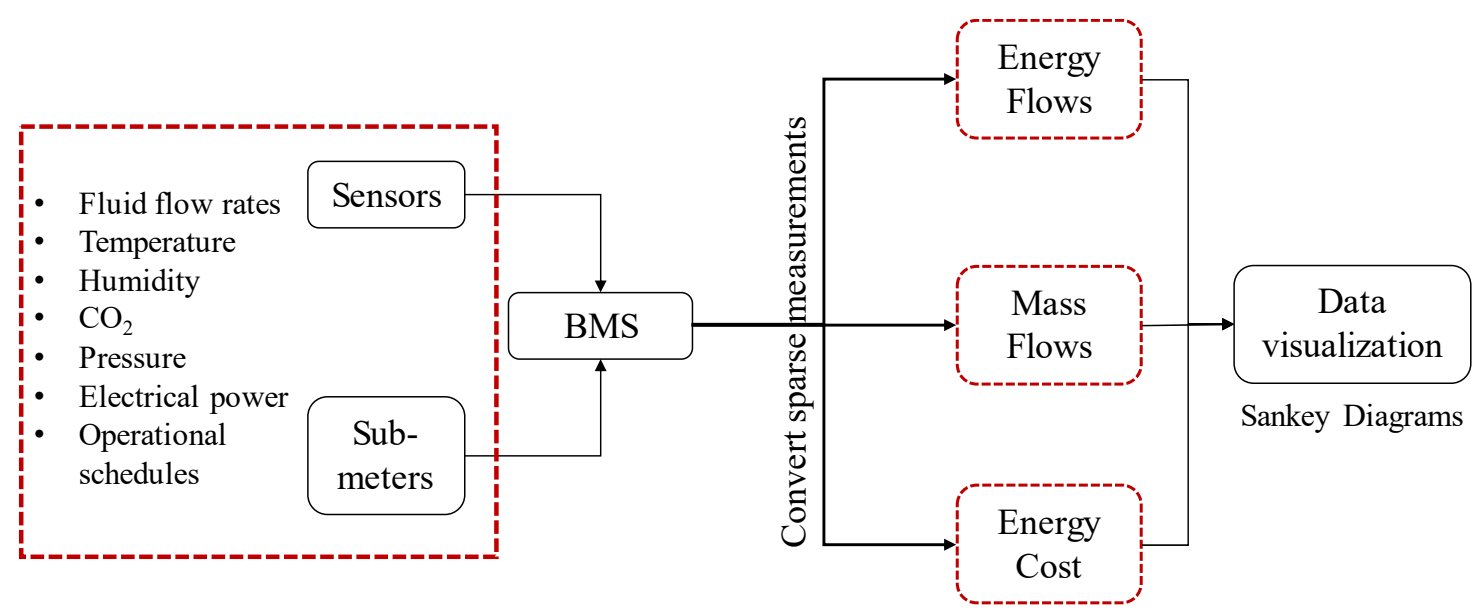

Figure 5.3: Flowchart showing the process to convert historical data to estimated energy and mass flows and the corresponding cost and visualize using Sankey diagrams 


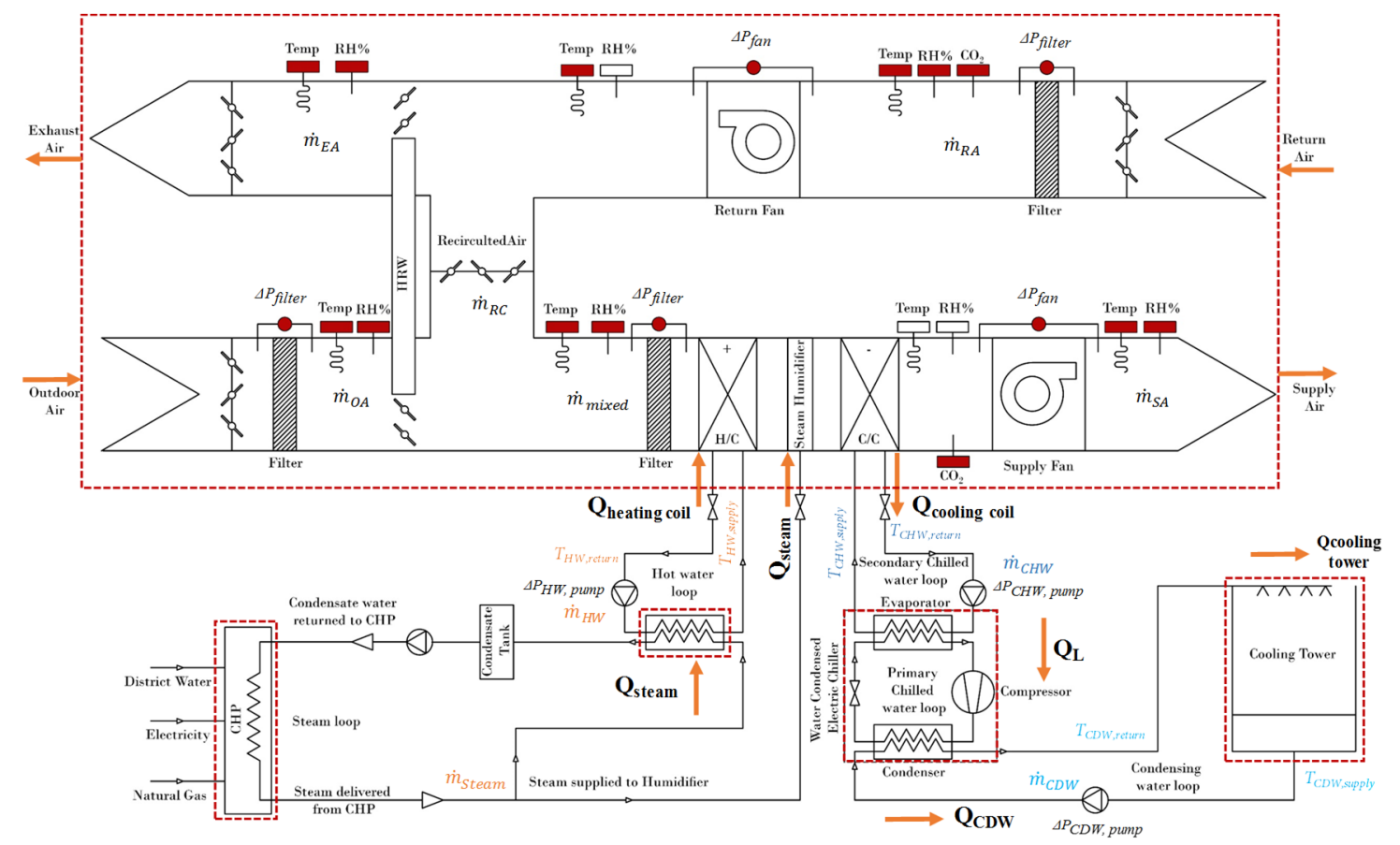

Figure 5.4: Typical AHU and plant loops of a commercial building showing actually measured sensors in the selected case study (highlighted in red color) and control volumes (within dotted lines)

The following sections aim to illustrate energy balances for typical HVAC equipment (i.e. boilers, cooling towers, chillers, pumps, and air handling unit (AHU) subsystems) of a commercial building. The following energy balance equations of each component were written in terms of final energy, making use of the following symbols: thermal load $Q$, positive for heating and negative for cooling, $\dot{m}$ is the mass flow rate of fluids, $C_{p}$ is the specific heat capacity, and $\rho, T$ and $h$ are the density, temperature and enthalpy of fluid in and out of the system, respectively. While, $P_{e l}$ and $\Delta P$ are the amount of electric energy consumption and differential pressure, which can be obtained from BMS. All units are in SI.

The equations rely on a set of assumptions. For instance, it was assumed that there was no internal energy change (i.e. steady-state) as the thermal capacity of the equipment 
is quite low compared to the energy flows. Moreover, the system does not rapidly change in operations (e.g. airflow rate) [224]. Furthermore, the heat flow through the AHU is several orders of magnitude higher than the heat loss from AHU to the surrounding and frictional losses, thus they were ignored in the current study. Furthermore, the pressure drop in the coils/heat exchangers were neglected from the current study.

Contrary to simulation-based studies, energy flows are often not readily available as readings from BMS. Instead, modern buildings measure fluid flow rates, temperature, humidity, and electrical power. Thus, a contribution of this work was to convert sparse measurements that are typically available in modern HVAC systems into a comprehensive set of energy flows that can be properly interpreted and visualized.

\subsubsection{Central Heating Plant (CHP)}

Central heating plants (CHP) are commonly used in many communities and campuses. The aim of the CHP is to reduce equipment size and cost, improve efficiency, and centralize operations. The steam produced from the CHP is distributed to buildings through network of pipes to use the thermal energy to provide heat to heating coils, radiators, radiant panels, and hot water. In the context of the selected case study, the steam delivered from the CHP passes through a heat exchanger that uses the thermal energy to provide heat to the hot water-glycol (HW) loop. The energy balance across the heat exchanger yields as shown in Equation (5.1). A portion of the steam delivered to the building is consumed directly by steam humidifier, which is discussed in Section 5.3.5.6. $Q_{\text {steam }}$ can also be calculated from the amount of energy added by heating coil to the air stream (discussed in Section 5.3.5.1) and frictional losses by hot water pump as in Equation (5.2). The frictional loss by the pumps is discussed in Section 5.3.4. 


$$
\begin{gathered}
Q_{\text {steam }}=\dot{m}_{\text {steam }} \times\left(h_{\text {Steam,supply }}-h_{\text {steam, return }}\right)=\dot{m}_{H W} \times\left(h_{H W, \text { supply }}-h_{H W, \text { return }}\right) \\
Q_{\text {steam }}=Q_{\text {heating coil }}-\sum_{H W, \text { pump }}
\end{gathered}
$$

where $\sum F_{H W, p u m p}$ is the thermal energy added by the hot water-glycol pump due to friction losses. There are two sources of steam mass loss, due to inefficiency of CHP and distribution losses through the network of pipes. These values were calculated based on a method developed in a companion paper [197].

\subsubsection{Cooling Towers}

Cooling towers are a heat rejection device that rejects waste heat to the atmosphere. The condensing water (CDW) carries heat from the condenser to the cooling tower to be cooled and returned to the condenser. This loop is called condensing water loop. Equation (5.3) is used to calculate the amount of energy extracted from the condensing water.

$$
Q_{C D W}=\dot{m}_{C D W} \times\left[T_{C D W, \text { return }} \times C_{p}\left(T_{C D W, \text { return }}\right)-T_{C D W, \text { supply }} \times C_{p}\left(T_{C D W, \text { supply }}\right)\right]
$$

The total amount of energy extracted by the cooling towers is calculated in Equation (5.4).

$$
Q_{\text {cooling tower }}=Q_{C D W}+\sum_{C D W, \text { pump }}+P_{e l}
$$

where $\sum F_{C D W, \text { pump }}$ is the thermal energy added by the condensing pump due to friction losses. While, $P_{e l}$ is the amount of electric energy consumption by the cooling tower fans.

\subsubsection{Chillers}

The refrigerant (water $60 \%$-propylene $40 \%$ ) glycol for the case study building) extracts heat from the chilled water (CHW) in the evaporator and transfers it to the condensing water by means of a vapor compression cycle. The current study focused on the overall system energy balance. Also, environmental heat loss is ignored. The energy 
balance across the chiller is shown in Equation (5.5) [231]. The coefficient of performance (COP) of the chiller is calculated in Equation (5.6).

$$
\begin{aligned}
& P_{e l}-Q_{C D W}+Q_{L}=0 \\
& C O P=\frac{Q_{L}}{Q_{C D W}-Q_{L}}
\end{aligned}
$$

where $Q_{C D W}$ is the amount of energy transferred from the condenser to the condensed water loop. $P_{e l}$ is the amount of electric energy consumption by the chillers. $Q_{L}$ is the amount of energy gained from the evaporator and calculated as shown in Equation (5.7). $Q_{L}$ can also be calculated from the amount of energy absorbed by the cooling coils (discussed in Section 5.3.5.1) and frictional losses from the CHW pump as shown in Equation (5.8).

$$
\begin{gathered}
Q_{L}=\dot{m}_{C H W} \times\left[T_{C H W, \text { return }} \times C_{p}\left(T_{C H W, \text { return }}\right)-T_{C H W, \text { supply }} \times C_{p}\left(T_{C H W, \text { supply }}\right)\right] \\
Q_{L}=Q_{\text {cooling coil }}+\sum_{C H W, \text { pump }}
\end{gathered}
$$

where $\sum F_{C H W \text { pump }}$ is the thermal energy added by the chilled water pump due to friction losses.

\subsubsection{Pumps}

The pumps are used to increase the pressure and temperature of the return fluids in the chilled water, condensing water, and steam loops. The energy balance through the pump is expressed in Equation (5.9).

$$
P_{\text {el }}=\dot{m}_{\text {reference }} \times\left(\frac{\Delta P_{\text {pump }}}{\rho_{\text {reference }}}\right)+\sum F
$$

where $\sum F$ is the friction losses through the pump. The subscript "reference" is used to refer to the type of fluid passing through the pumps (i.e. steam, hot water, chilled water, or condensate water). The efficiency of the pump can be calculated as shown in Equation $(5.10)$. 


$$
\eta_{\text {pump }}=\frac{\dot{m}_{\text {reference }} \times \frac{\Delta P_{\text {pump }}}{\rho_{\text {reference }}}}{P_{\text {el }}}
$$

where $P_{e l}$ is the amount of electric energy consumption by the pump.

\subsubsection{Air Handling Unit (AHU)}

The air handling unit is a part of the HVAC system that is used to condition and distribute air to building zones. In the context of the selected case study (the Canal Building), the air handler contains supply and return fans, heating and cooling coils, filters, humidifier, economizer, heat recovery wheel (HRW), and dampers [232]. The energy balance for the AHU is expressed in Equation (5.11) [233]. The enthalpy of air (reference of $0^{\circ} \mathrm{C}$ and $0 \% \mathrm{RH}$ ) was used in the following energy balance equations.

$$
\left(\dot{m}_{R A} \times h_{R A}\right)+\left(\dot{m}_{O A} \times h_{O A}\right)+Q_{\text {recovery }}+Q_{i n}=\left(\dot{m}_{S A} \times h_{\text {supply }}\right)+\left(\dot{m}_{E A} \times h_{E A}\right)+L
$$

where the subscripts $R A, O A, S A$, and $E A$ are return, outdoor, supply, and exhaust air, respectively. The term $Q_{\text {in }}$ refers to the amount of instantaneous energy added or extracted from the system, thus it can be positive for heating and negative for cooling. While $Q_{\text {recovery }}$ refers to the instantaneous recovered energy by the heat recovery wheel and $L$ is the losses in the system (e.g. frictional losses through AHU ducts and heat transfer across the walls of the AHU). Moreover, the AHU is equipped with a modulation damper to control the recirculated air. The following discussion focuses on the amount of energy added or extracted and recovered in the AHU.

\subsubsection{Heat Exchangers}

Heat exchangers (such as heating and cooling coils) are devices used to transfer heat between one or more fluids. Heat exchangers do not use energy but destroy exergy (second-law of thermodynamics) [220]. For the cooling coils, heat from the air stream 
passing through the cooling coils is absorbed by the chilled water and returns to the chiller evaporator to be cooled. This loop is called the secondary chilled water loop and is driven by pumps. The amount of energy extracted by the cooling coil is shown in Equation (5.12). The enthalpy was used in this equation to account for sensible and latent energy.

$$
\begin{aligned}
Q_{\text {cooling coil }}= & \dot{m}_{C H W} \times\left[T_{C H W, \text { return }} \times C_{p}\left(T_{C H W, \text { return }}\right)-T_{C H W, \text { supply }} \times C_{p}\left(T_{C H W, \text { supply }}\right)\right] \\
& =\dot{m}_{S A} \times\left(h_{S A, i n^{-}} h_{S A, \text { out }}\right)
\end{aligned}
$$

On the mass balance side, some of the vapor in the air condenses on the cooling coil surface leading to removal of vapor from humid air. The amount of vapor condensate from latent cooling is expressed in Equation (5.13).

$$
\dot{m}_{\text {condensation }}=\dot{m}_{\text {dry air }} \times\left(W_{\text {in }}-W_{\text {out }}\right)
$$

where $W$ is the humidity ratio entering and leaving the cooling coil. The energy balance across the heating coil can be expressed as shown in Equation (5.14). Heat is transferred from the hot glycol-water to the air stream running through the heating coil. The hot water return from the heating coil runs through another heat exchanger by means of pumps.

$$
\begin{aligned}
Q_{\text {heating coil }} & =\dot{m}_{H W} \times\left[T_{H W, \text { supply }} \times C_{P}\left(T_{H W, \text { supply }}\right)-T_{H W, \text { return }} \times C_{P}\left(T_{H W, \text { return }}\right)\right] \\
& =\dot{m}_{S A} \times\left(h_{S A, \text { in }}-h_{S A, \text { out }}\right)
\end{aligned}
$$

\subsubsection{Heat Recovery Wheel (HRW)}

In this study, an enthalpy wheel was discussed, which allows both sensible and latent energy to be recovered from the exhaust air stream. Equations (5.15) and (5.16) show sensible and latent energy recovered by energy wheel, respectively.

$$
\begin{gathered}
Q_{\text {recovery,sensible }}=\dot{m}_{R A} \times(100-\% O A) \times\left[T_{E A} \times C_{P}\left(T_{E A}\right)-T_{R A} \times(1-\% O A) \times C_{P}\left(T_{R A}\right)\right] \\
Q_{\text {recovery,latent }}=\dot{m}_{R A} \times(100-\% O A) \times\left(h_{E A}-h_{R A}\right)
\end{gathered}
$$


where $h_{E A}$ and $h_{R A}$ are the specific enthalpy for exhaust and return air, respectively. While the $\% O A$ is the outdoor air fraction. The enthalpy $h$ used in this equation includes moisture and is calculated as shown in Equation (5.17) [231].

$$
h_{\text {moist }}=h_{d a}+W \times h_{g} \approx T+W(2501+1.805 T)
$$

where $W$ is the humidity ratio, and $h_{d a}$ and $h_{g}$ are the specific enthalpy for dry air and saturated water vapor, respectively.

\subsubsection{Fans}

In this study, a variable volume fan was used. The aim is to calculate the efficiency of the fan and to determine the state of the air exiting the fan. The rate of electrical energy consumption by the fan's motor is shown in Equation (5.18).

$$
P_{e l}=\frac{W_{f a n}}{\eta_{f a n}}=\frac{\dot{m} \times \frac{\Delta P_{f a n}}{\rho}}{\eta_{f a n}}
$$

where $W_{\text {fan }}$ is the rate of energy transfer by work from fan to air and $\eta_{\text {fan }}$ is the efficiency of fan's motor. On the other hand, the frictional losses through the fan can be determined as shown in Equation (5.19).

$$
P_{e l}=\dot{m} \times\left(\frac{\Delta P_{\text {fan }}}{\rho_{\text {air }}}\right)+\sum F
$$

where $\sum F$ is the friction losses through the fan. Moreover, the state of air leaving the fan is expressed in Equation (5.20).

$$
h_{\text {out }}-h_{\text {in }}=\frac{P_{e l} \times\left[\eta_{\text {fan }}+\beta_{\text {motor }} \times\left(1-\eta_{\text {fan }}\right)\right]}{\dot{m}_{\text {air }}}
$$

where $\beta_{\text {motor }}$ is the fraction of heat loss from motor that is transferred to the air stream in the AHU as shown in Equations (5.21) and (5.22). 


$$
\begin{gathered}
\beta_{\text {motor }}=\frac{\dot{Q}_{\text {motor-to-air }}}{\dot{Q}_{\text {motor }}} \\
\dot{Q}_{\text {motor }}=P_{e l}-W_{f a n}
\end{gathered}
$$

where $\dot{Q}_{\text {motor }}$ is the rate of heat addition from the fan to the air stream due to frictional losses. While, $\dot{Q}_{\text {motor-to-air }}$ depends on the fan location in the air stream and can be obtained as shown in Equation (5.23).

$$
\dot{Q}_{\text {motor-to-air }}=\frac{f \times P_{e l}}{\eta_{f a n}}
$$

The factor $f$ can be determined based on the location of the fan in the air stream [234].

\subsubsection{Filters}

The purpose of air filters is to remove the dust from both the ventilation air and the recirculated air in the air handler. In a typical air handling units, air filters consume approximately 10 to 70 percent of the total fan energy consumption [235]. This study focused on evaluating air filter based on their resistance to air flow. Thus, the frictional losses can be calculated as shown in Equation (5.24).

$$
\sum F=\dot{m}_{\text {air }} \times\left(\frac{\Delta P_{\text {filter }}}{\rho_{\text {air }}}\right)
$$

\subsubsection{Outdoor Air Fraction}

The percentage or fraction of outside air can be estimated by using carbon dioxide $\left(\mathrm{CO}_{2}\right)$ concentration of the supply, return, and outdoor air as shown in Equation (5.25) [236].

$$
O A \%=\frac{\mathrm{CO}_{2, \text { supply,air }}{ }^{-} \mathrm{CO}_{2, \text { return,air }}}{C O_{2, \text { outdoor,air }}{ }^{-C O_{2, \text { return, air }}}}
$$

In some cases, $\mathrm{CO}_{2}$-based calculation of outdoor air fraction could encounter some errors as in the case of the Canal Building. Thus, an alternate approach can be used to estimate 
outdoor air fraction using enthalpy of air, as in Equation (5.26).

$$
O A \%=\frac{\dot{m}_{R A} \times \eta \times\left(h_{R A}-h_{E A}\right)+}{\left.\dot{m}_{M A} \times\left[h_{R A} \times(1-\eta)+\left(h_{E A} \times \eta\right)-h_{M A}\right)\right]}
$$

where subscript $M A$ refers to the mixed air and $\eta$ is the efficiency of the HRW.

\subsubsection{Humidifiers}

The humidification process is the addition of moisture or water vapor to the air stream. This could be achieved by introducing water vapor or by spraying fine droplets of water that evaporate into the air stream [237]. In the building case study that follows, humidification was achieved by injecting steam into the airstream. The humidification load is computed by Equation (5.27).

$$
\text { Humidification Load }=\dot{m}_{\text {air }} \times\left(h_{\text {out }}-h_{\text {in }}\right)=\dot{m}_{\text {steam }} \times h_{\text {steam }}
$$

The mass flow rate of steam $\dot{m}_{\text {steam }}$ is obtained from the amount of moisture $W$ added to the air stream as shown in Equation (5.28).

$$
\dot{m}_{\text {steam }}=\dot{m}_{\text {supply air }} \times\left(W_{\text {out }}-W_{\text {in }}\right)
$$

where $W$ is the humidity ratio entering and leaving the humidifier.

\subsection{Case Study}

The Canal Building (CB) is a seven-story mixed-use academic building located in Ottawa, Canada with total floor area about $8,000 \mathrm{~m}^{2}$ as shown in Figure 5.5. Ottawa's climate is a humid continental climate, characterized by hot-humid summers and coldhumid winter days. The average historical heating degree days (HDD) and cooling degree days (CDD) for Ottawa are 4434 and 267, respectively, both in SI units [179]. Figure 5.6 shows actual weather data of Ottawa, Canada in 2015. The 2015 HDD and CDD were 4500 
and 385 , respectively.

The building began its operation in 2011, including a large variety of functional space such as private offices, open-plan offices, lecture rooms, computer labs, design labs, research labs, conference rooms and other facility rooms. The building is also equipped with more than 2500 sensors to collect data required to inspect sub-hourly energy consumption for each floor and HVAC system components.

This building is equipped with four AHUs. Two small AHUs are designated for the mechanical rooms, while the rest of the building is conditioned by two much larger separate AHUs. This study focused on analyzing the performance of one of the main AHUs installed and its impact on the plant loops. Table 5.1 shows a summary of the air handling unit and plant loops specifications. The air distribution system is single-duct variable air volume (VAV) with reheat coils. Moreover, some of the perimeter zones are equipped with radiant heating panels. 


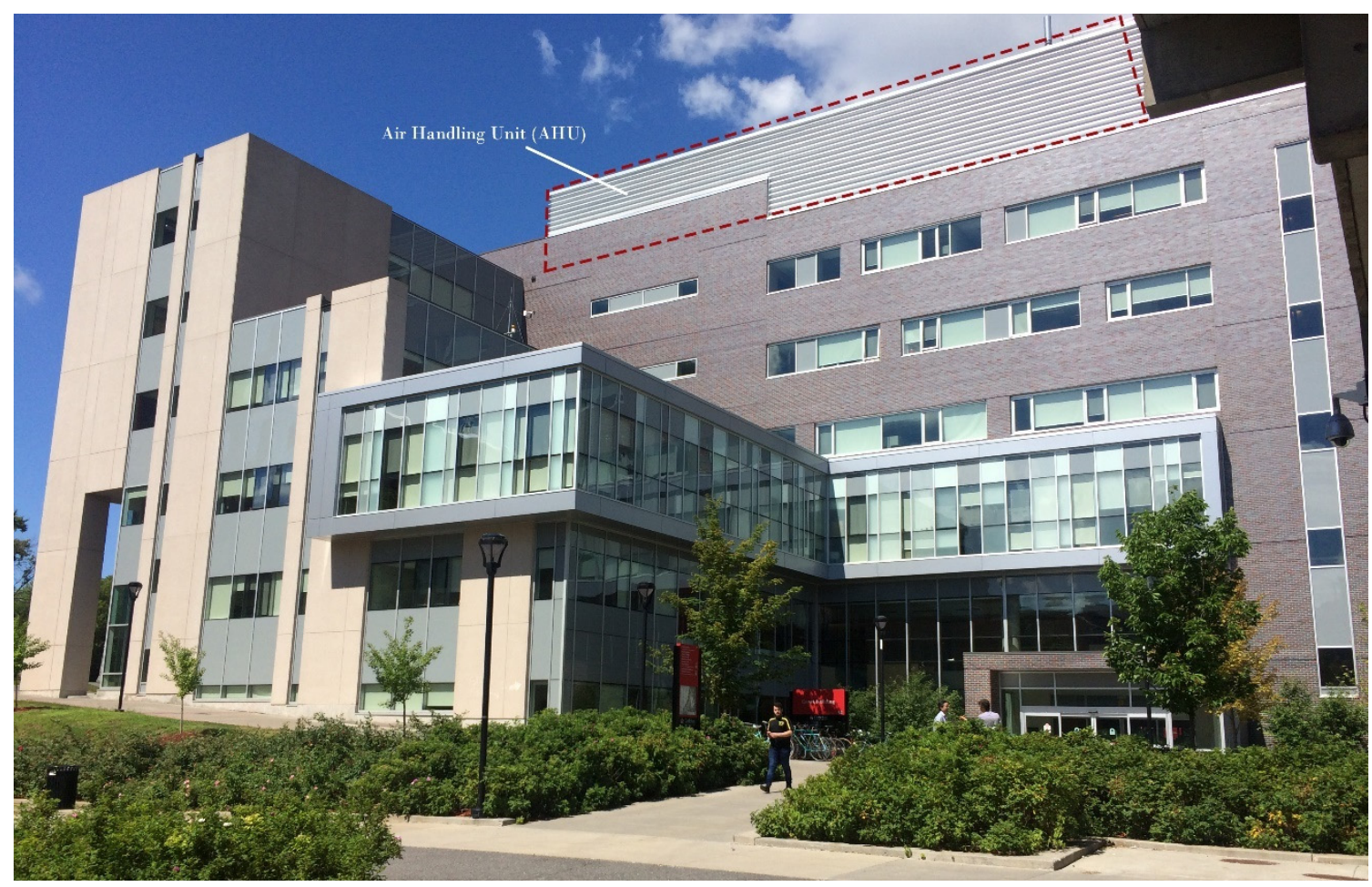

Figure 5.5: Canal Building

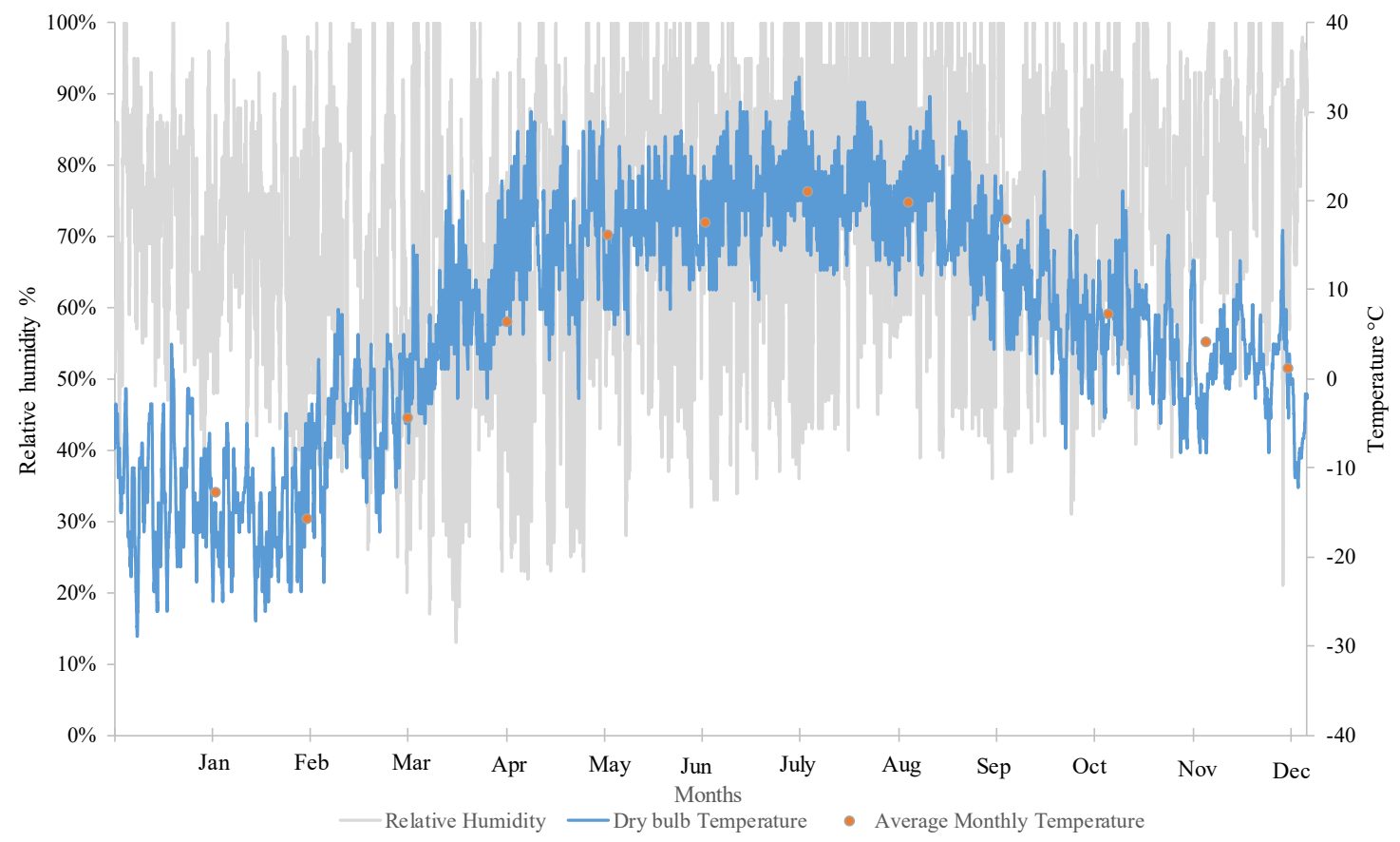

Figure 5.6: Actual weather data of Ottawa, Canada in 2015 
Table 5.1: Air handling unit and plant loops details summary

\begin{tabular}{|c|c|c|}
\hline \multicolumn{2}{|c|}{ HVAC components } & Specifications \\
\hline \multirow{2}{*}{$\begin{array}{l}\text { Heating } \\
\text { System }\end{array}$} & $\begin{array}{l}\text { The building relies on } \\
\text { the steam generated at a } \\
\text { central plant (three } \\
\text { boilers) }\end{array}$ & Average boilers efficiency is 80 percent \\
\hline & $\begin{array}{l}\text { Heat Exchanger (steam } \\
\& \text { hot glycol water) }\end{array}$ & $\begin{array}{l}\text { Fluid: Glycol/water / } 315 \mathrm{Gpm}(20 \mathrm{~L} / \mathrm{s}) / \\
\text { Entering Fluid Temp } 71.1^{\circ} \mathrm{C} / \text { Leaving } \\
\text { Fluid Temp } 82.2^{\circ} \mathrm{C} / \text { Load } 3255 \mathrm{Lbs} / \mathrm{hr} \\
\text { [ 993 kW] }\end{array}$ \\
\hline \multirow{2}{*}{$\begin{array}{l}\text { Cooling } \\
\text { System }\end{array}$} & Chillers & $\begin{array}{l}\text { Two chillers (ElectricEIRChiller } \\
\text { Centrifugal Carrier 19XR 1407kW/ } \\
6.04 \text { COP/ VSD) }\end{array}$ \\
\hline & Cooling Towers & Air-cooled heat exchanger \\
\hline \multirow{6}{*}{$\begin{array}{l}\text { Air Handling } \\
\text { Unit }\end{array}$} & Supply Fan & $\begin{array}{l}46000 \mathrm{CFM}(21709 \mathrm{~L} / \mathrm{s}) / 75 \mathrm{HP}(56 \mathrm{~kW}) \\
\text { / } 60.7 \mathrm{Bhp}(45.3 \mathrm{~kW}) / 1750 \mathrm{Rpm}\end{array}$ \\
\hline & Return Fan & $\begin{array}{l}46000 \text { CFM (21709 L/s) / } 50 \text { HP (37.3 } \\
\mathrm{kW}) / 40 \mathrm{Bhp}(30 \mathrm{~kW}) / 1750 \mathrm{Rpm}\end{array}$ \\
\hline & Heating Coil & $\begin{array}{l}\text { Fluid: Glycol/water } / 88 \mathrm{Gpm}(5.55 \mathrm{~L} / \mathrm{s}) / \\
\text { Entering Fluid Temp } 7.2^{\circ} \mathrm{C} / \text { Leaving } \\
\text { Fluid Temp } 15.5^{\circ} \mathrm{C}\end{array}$ \\
\hline & Cooling Coil & $\begin{array}{l}\text { Fluid: } 60 \% \text { water - } 40 \% \text { propylene glycol } \\
\text { / } 229 \mathrm{Gpm}(14.45 \mathrm{~L} / \mathrm{s}) / \text { Entering Fluid } \\
\text { Temp } 82.2^{\circ} \mathrm{C} / \text { Leaving Fluid Temp } \\
71.1^{\circ} \mathrm{C}\end{array}$ \\
\hline & Steam Humidifier & $\begin{array}{l}7000 \mathrm{CFM}(3303 \mathrm{~L} / \mathrm{s}) / \mathrm{Load} 200 \mathrm{Lbs} / \mathrm{hr} \\
{[\sim 61 \mathrm{~kW}]}\end{array}$ \\
\hline & Heat Recovery Wheel & $\begin{array}{l}\text { Summer conditions: } \\
\text { entering air temp. } 32.2^{\circ} \mathrm{C} \text {, leaving air } \\
\text { temp. } 25^{\circ} \mathrm{C} \text {, efficiency sensible } 86.2 \% \text {, } \\
\text { energy saved } 119 \mathrm{MBH}(34.9 \mathrm{MW}) \text {. }\end{array}$ \\
\hline
\end{tabular}




\begin{tabular}{|l|l|l|}
\hline & & $\begin{array}{l}\text { Winter conditions: } \\
\text { entering air temp. }-23.3^{\circ} \mathrm{C}, \text { leaving air } \\
\text { temp. } 16^{\circ} \mathrm{C}, \text { efficiency sensible } 86.2 \%, \\
\text { energy saved } 110.9 \mathrm{MBH}(32.5 \mathrm{MW}) .\end{array}$ \\
\hline \multirow{3}{*}{ Pumps } & Heating System & $\begin{array}{l}315 \mathrm{GPM}(148.6 \mathrm{~L} / \mathrm{s}) / 10 \mathrm{HP}(7.45 \mathrm{~kW}) / \\
\text { efficiency } 70.3 \%\end{array}$ \\
\cline { 2 - 3 } & Chilled Water & $\begin{array}{l}960 \mathrm{GPM}(453 \mathrm{~L} / \mathrm{s}) / 50 \mathrm{HP}(37.3 \mathrm{~kW}) / \\
\text { efficiency } 76.9 \%\end{array}$ \\
\cline { 2 - 3 } & Condensing Water & $\begin{array}{l}1200 \mathrm{GPM}(566.3 \mathrm{~L} / \mathrm{s}) / 50 \mathrm{HP}(37.3 \mathrm{~kW}) \\
/ \text { efficiency } 77 \%\end{array}$ \\
\hline
\end{tabular}

The following discussion focuses on providing information on the AHU, hot water, chilled water, and condensing water loops sequence of operations.

\section{- Air handling unit sequence of operations are summarized as follows:}

The air handling units are run in conjunction with zone controls (VAV-reheat coils and radiant panels), the heating plant, and the cooling plant. Requests are received from the zone controllers to increase/decrease the supply air temperature setpoint. Space temperature setpoints are mostly $22^{\circ} \mathrm{C}$ and $24^{\circ} \mathrm{C}$ for heating and cooling, respectively. The following discussion provides information on the operation of AHU components.

1. Outdoor air dampers position is modulated to maintain supply air $\mathrm{CO}_{2}$ set point $(800 \mathrm{ppm})$ and to maintain supply air set point $\left(13-20^{\circ} \mathrm{C}\right)$ (only in free cooling). Free cooling is enabled when OAT is less than $18^{\circ} \mathrm{C}$. The position of the dampers is based on the greater request to maintain $\mathrm{CO}_{2}$ or supply air temperature setpoints.

2. Exhaust and return air dampers position is modulated based on the position of outdoor air dampers. Moreover, the exhaust, return, and outdoor dampers are 
modulated to maintain mixed air temperature at $18.3^{\circ} \mathrm{C}$. The minimum mixed air temperature that could be reached is $-2.2^{\circ} \mathrm{C}$.

3. The cooling and heating coils operate when the supply air temperature setpoint is higher than $20^{\circ} \mathrm{C}$ and below $13^{\circ} \mathrm{C}$, respectively.

4. Steam humidifier operates to maintain return air humidity at $30 \%$ and limit the supply air humidity to a maximum of $75 \%$. The steam humidifier is disabled when OAT is greater than $10^{\circ} \mathrm{C}$. The high limit controller is disabled when the OAT is above $24^{\circ} \mathrm{C}$.

5. The supply fan is modulated to maintain $2 / 3$ of supply air static pressure at its setpoint (373 Pa) $(\sim 10,800 \mathrm{~L} / \mathrm{s})$.

6. The return fan is modulated to maintain flow difference of $(1770 \mathrm{~L} / \mathrm{s})$ between supply air flow and return air flow.

7. The heat recovery wheel (HRW) operates when the exhaust air temperature is higher than $15^{\circ} \mathrm{C}$. Heat wheel bypass dampers are used to protect the wheel from freezing. When the OAT drops below the frost threshold, the wheel speed is modulated to maintain the relative humidity of the exhaust air at less than $90 \%$.

- Steam \& Hot water loops sequence of operations are summarized as follows:

1. The energy management system (EMS) sends a request to modulate the steam valves to enable the steam to pass through the heat exchanger to transfer heat to the hot water. The EMS also sends a request to operate the hot water pumps when the heating coil valve is opened.

2. The steam valves are modulated to maintain hot water supply temperature setpoint $\left(51.5-81.5^{\circ} \mathrm{C}\right)$. 
3. The hot water loop is designed to have one pump running and one pump operating as a standby pump.

4. The pump speed is modulated to maintain differential pressure setpoint ( $2-8$ Psi) between supply and return hot water.

5. When only one pump is operating, the minimum speed of the pump motor as commanded by the variable frequency drive (VFD) is $40 \%$.

- Chilled and condensing water loops sequence of operations are summarized as

\section{follows:}

1. The EMS sends a request to operate the pumps, chillers, and cooling tower fans when the cooling coil valve is opened. The cooling system stops operating if the OAT is below $10^{\circ} \mathrm{C}$.

2. If the chilled water supply common header temperature rises more than $1{ }^{\circ} \mathrm{C}$ above its setpoint of $5.6^{\circ} \mathrm{C}$ for more than 10 minutes, then the EMS will start the lag chiller in sequence to maintain the header temperature setpoint.

3. If both chillers are running at less than $35 \%$ of the rated capacity for more than 10 minutes, then the lag chiller goes off-line first, its related chilled water pump next and finally its condenser water pump with a 3-minutes time delay.

4. The cooling towers leaving water temperature setpoint is adjusted based on the number of chillers required to satisfy cooling demand. The setpoint is $22^{\circ} \mathrm{C}$ with one chiller on-line and $25^{\circ} \mathrm{C}$ with both chillers on-line.

5. The condenser water circulating pumps shall run at two different fixed speeds based on the number of the chillers on-line. 
6. Ramping up and down of the cooling towers fan speed by variable frequency drive (VFD) shall be limited between $25 \%$ and $100 \%$.

\subsection{Data Acquisition}

This section discusses the challenges in obtaining the data from BMS. The current study focused on historical data for four months in winter (January-April 2015) and summer (May-September 2015). The study relied on existing sensors and sub-meters installed in the AHU and plant loops. The Canal Building is equipped with abundant sensors and sub-meters $(\sim 365)$ to inspect the performance of each component in the AHU and plant loops. However, this building is supplying cooling to itself and one of the adjacent buildings. Thus, the first step was to identify the chiller energy consumption assigned to the building. This was done by obtaining data for chilled water flow rate to the Canal Building and the total flow rate for all supplied buildings as shown in Equation (5.29). On the other side, the steam energy consumption was metered for each building, including the Canal Building.

$$
\begin{gathered}
\text { Chiller consumption } \\
\text { for } C B
\end{gathered}=\begin{gathered}
\text { Chillers total } \\
\text { consumption }
\end{gathered} \times\left(\begin{array}{c}
\text { Flow rate for } C B \\
\begin{array}{c}
\text { Total flow rate for all buildings } \\
\text { sharing the same chillers }
\end{array}
\end{array}\right)
$$

During the summer season that was studied, the AHU operated for 1840 hours and during the winter season it was operating for 2975 hours. The number of operating hours refers to the valid data points that were used in the current study to analyze data for heating and cooling seasons only (excluding shoulder seasons). Some modest errors $(\sim 5$ percent) were associated with some of the sensors (e.g. return, exhaust, mixed, and outdoor temperature, supply and return air $\mathrm{CO}_{2}$, and differential pressure across supply and return fans) such as missing or inconsistent data points. For instance, the supply air $\mathrm{CO}_{2}$ sensor 
reset to outdoor value if the sensing value exceeds the maximum threshold. There are many sources of measurement errors such as sensor accuracy, hysteresis, offset, noise, repeatability, resolution, and threshold. The error that is related to the variation in data due to precision and resolution is calculated as shown in Equation (5.30). Sensor data is only considered erroneous if it is missing or inconsistent. Data points are considered inconsistent as they differ dramatically from the rest of the data. These points are often referred to as outliers. Outlier is defined as data points that are more than 1.5 interquartile ranges (IQRs) below the first quartile (25th percentile) or above the third quartile (75th percentile).

$$
\text { Sensor error }=\frac{s}{n} \times 100
$$

where, $s$ is the number of data points obtained from sensors that contain errors (such as missing or inconsistent data points) and $n$ is the number of data samples. Table 5.2 shows sensor errors, upper and lower limits, and calibration of each sensor and sub-meter installed in the AHU and plant loops. Moreover, most of the sensors have different sensing frequencies. Some sensors pull the data every 5 minutes, and some other sensors pull the data on larger sample intervals (i.e. 10 to 15 minutes). In the current study, interpolation was performed to generate missing values for short time-periods ( $<6$ hours). While for long time-periods, the data were excluded from the analysis.

Table 5.2: Sensors and sub-meters' details summary

\begin{tabular}{|c|c|c|c|c|c|}
\hline & $\begin{array}{l}\text { Sensor/sub- } \\
\text { meter type }\end{array}$ & Calibration & Lower limit & Upper limit & Sensor error \\
\hline Steam & Pressure & $0 \mathrm{Psi}$ & 6 Psi & 20 Psi & $0.42 \%$ \\
\hline \multirow{2}{*}{$\begin{array}{l}\text { Heat } \\
\text { Exchanger } \\
\text { (steam \& } \\
\text { hot water) }\end{array}$} & $\begin{array}{l}\text { Hot water } \\
\text { supply } \\
\text { temperature }\end{array}$ & $0^{\circ} \mathrm{C}$ & $51.5^{\circ} \mathrm{C}$ & $81.5^{\circ} \mathrm{C}$ & $2.57 \%$ \\
\hline & $\begin{array}{l}\text { Hot water } \\
\text { return }\end{array}$ & $0.5^{\circ} \mathrm{C}$ & N/A & N/A & $2.33 \%$ \\
\hline
\end{tabular}




\begin{tabular}{|c|c|c|c|c|c|}
\hline & temperature & & & & \\
\hline \multirow{4}{*}{$\begin{array}{l}\text { Cooling } \\
\text { tower }\end{array}$} & Fluid flow rate & 0 GPM & $\mathrm{N} / \mathrm{A}$ & $\mathrm{N} / \mathrm{A}$ & $0.26 \%$ \\
\hline & $\begin{array}{l}\text { Condensing } \\
\text { water supply } \\
\text { temperature }\end{array}$ & $0^{\circ} \mathrm{C}$ & N/A & $\mathrm{N} / \mathrm{A}$ & $0.88 \%$ \\
\hline & $\begin{array}{l}\text { Condensing } \\
\text { water return } \\
\text { temperature }\end{array}$ & $0^{\circ} \mathrm{C}$ & N/A & $\mathrm{N} / \mathrm{A}$ & $1.01 \%$ \\
\hline & $\begin{array}{l}\text { Fans power } \\
\text { consumption }\end{array}$ & $0 \mathrm{~kW}$ & N/A & $\mathrm{N} / \mathrm{A}$ & $0.17 \%$ \\
\hline \multirow{4}{*}{ Chiller } & Fluid flow rate & 0 GPM & N/A & $\mathrm{N} / \mathrm{A}$ & $0.21 \%$ \\
\hline & $\begin{array}{l}\text { Chilled water } \\
\text { supply } \\
\text { temperature }\end{array}$ & $0^{\circ} \mathrm{C}$ & $4^{\circ} \mathrm{C}$ & $12^{\circ} \mathrm{C}$ & $0.24 \%$ \\
\hline & $\begin{array}{l}\text { Chilled water } \\
\text { return } \\
\text { temperature }\end{array}$ & $0^{\circ} \mathrm{C}$ & N/A & $\mathrm{N} / \mathrm{A}$ & $0.31 \%$ \\
\hline & $\begin{array}{l}\text { Power } \\
\text { consumption }\end{array}$ & $0 \mathrm{~kW}$ & N/A & $\mathrm{N} / \mathrm{A}$ & $0.17 \%$ \\
\hline \multirow{2}{*}{$\begin{array}{l}\text { Hot water } \\
\text { pump }\end{array}$} & $\begin{array}{l}\text { Differential } \\
\text { pressure }\end{array}$ & 0 Psi & 2 Psi & 24 Psi & $2.13 \%$ \\
\hline & $\begin{array}{l}\text { Power } \\
\text { consumption }\end{array}$ & $0 \mathrm{~kW}$ & N/A & $\mathrm{N} / \mathrm{A}$ & $0.07 \%$ \\
\hline \multirow{2}{*}{$\begin{array}{l}\text { Condensing } \\
\text { water } \\
\text { pump }\end{array}$} & $\begin{array}{l}\text { Differential } \\
\text { pressure }\end{array}$ & 0 Psi & N/A & $\mathrm{N} / \mathrm{A}$ & $0.07 \%$ \\
\hline & $\begin{array}{l}\text { Power } \\
\text { consumption }\end{array}$ & $0 \mathrm{~kW}$ & N/A & N/A & $0.17 \%$ \\
\hline \multirow{2}{*}{$\begin{array}{l}\text { Chilled } \\
\text { water } \\
\text { pump }\end{array}$} & $\begin{array}{l}\text { Differential } \\
\text { pressure }\end{array}$ & 0 Psi & N/A & $\mathrm{N} / \mathrm{A}$ & $2.06 \%$ \\
\hline & $\begin{array}{l}\text { Power } \\
\text { consumption }\end{array}$ & $0 \mathrm{~kW}$ & N/A & N/A & $2.28 \%$ \\
\hline \multirow{5}{*}{ AHU } & $\begin{array}{l}\text { Return air } \\
\text { temperature }\end{array}$ & $0^{\circ} \mathrm{C}$ & N/A & $\mathrm{N} / \mathrm{A}$ & $4.86 \%$ \\
\hline & $\begin{array}{l}\text { Return air } \\
\text { relative } \\
\text { humidity }\end{array}$ & $3 \%$ & $15 \%$ & $65 \%$ & $0.01 \%$ \\
\hline & Return air $\mathrm{CO}_{2}$ & $0 \mathrm{ppm}$ & 200 & 1500 & $55 \%$ \\
\hline & $\begin{array}{l}\text { Return fan } \\
\text { differential } \\
\text { pressure }\end{array}$ & $0 \mathrm{~Pa}$ & N/A & N/A & $10 \%$ \\
\hline & $\begin{array}{l}\text { Return fan } \\
\text { power } \\
\text { consumption }\end{array}$ & $0 \mathrm{~kW}$ & N/A & N/A & $0.38 \%$ \\
\hline
\end{tabular}




\begin{tabular}{|c|c|c|c|c|}
\hline $\begin{array}{l}\text { Return air flow } \\
\text { rate }\end{array}$ & $0 \mathrm{~L} / \mathrm{s}$ & N/A & $\mathrm{N} / \mathrm{A}$ & $10 \%$ \\
\hline $\begin{array}{l}\text { Exhaust air } \\
\text { temperature }\end{array}$ & $0^{\circ} \mathrm{C}$ & $1^{\circ} \mathrm{C}$ & $50^{\circ} \mathrm{C}$ & $0.66 \%$ \\
\hline $\begin{array}{l}\text { Exhaust air } \\
\text { relative } \\
\text { humidity }\end{array}$ & $-2 \%$ & N/A & $\mathrm{N} / \mathrm{A}$ & $0.01 \%$ \\
\hline $\begin{array}{l}\text { Outdoor air } \\
\text { temperature }\end{array}$ & $0^{\circ} \mathrm{C}$ & N/A & $\mathrm{N} / \mathrm{A}$ & $0.52 \%$ \\
\hline $\begin{array}{l}\text { Outdoor air } \\
\text { relative } \\
\text { humidity }\end{array}$ & $0 \%$ & N/A & $\mathrm{N} / \mathrm{A}$ & $0.03 \%$ \\
\hline $\begin{array}{l}\text { Mixed air } \\
\text { temperature }\end{array}$ & $0^{\circ} \mathrm{C}$ & N/A & $\mathrm{N} / \mathrm{A}$ & $20 \%$ \\
\hline $\begin{array}{l}\text { Mixed air } \\
\text { relative } \\
\text { humidity }\end{array}$ & $3 \%$ & N/A & N/A & $1.65 \%$ \\
\hline Supply air $\mathrm{CO}_{2}$ & $0 \mathrm{ppm}$ & N/A & $\mathrm{N} / \mathrm{A}$ & $5 \%$ \\
\hline $\begin{array}{l}\text { Supply air } \\
\text { temperature } \\
\text { after coils }\end{array}$ & $0^{\circ} \mathrm{C}$ & N/A & N/A & $0.76 \%$ \\
\hline $\begin{array}{l}\text { Supply air } \\
\text { relative } \\
\text { humidity after } \\
\text { coils }\end{array}$ & $2 \%$ & N/A & $\mathrm{N} / \mathrm{A}$ & $0.01 \%$ \\
\hline $\begin{array}{l}\text { Supply fan } \\
\text { differential } \\
\text { pressure }\end{array}$ & $0 \mathrm{~Pa}$ & N/A & $625 \mathrm{~Pa}$ & $15 \%$ \\
\hline $\begin{array}{l}\text { Supply air } \\
\text { flow rate }\end{array}$ & $0 \mathrm{~L} / \mathrm{s}$ & $\mathrm{N} / \mathrm{A}$ & $\mathrm{N} / \mathrm{A}$ & $15 \%$ \\
\hline $\begin{array}{l}\text { Supply fan } \\
\text { power } \\
\text { consumption }\end{array}$ & $0 \mathrm{~kW}$ & N/A & N/A & $0.16 \%$ \\
\hline $\begin{array}{l}\text { Supply air } \\
\text { temperature }\end{array}$ & $0^{\circ} \mathrm{C}$ & N/A & N/A & $6 \%$ \\
\hline $\begin{array}{l}\text { Supply air } \\
\text { relative } \\
\text { humidity }\end{array}$ & $1.5 \%$ & $0 \%$ & $85 \%$ & $2.8 \%$ \\
\hline
\end{tabular}

Another challenge occurred from temperature sensor errors caused by thermal stratification [238]. This problem occurred when mixing outdoor air with return air during summer and winter days. The thermal stratification pattern varies depending on the state 
of outdoor and return air, difference in velocity of air streams, and the geometry of the AHU. For example, during winter days and the HRW was not operating, it was found that the mixed air temperature before the heating coil and humidifier was higher than the temperature of return and outdoor air. Thus, an energy balance was performed for each component in the AHU to determine the state of the air as mentioned in Section 5.3.5. Moreover, it is essential to have temperature and relative humidity sensors installed before and after each component in the AHU to estimate the amount of latent energy injected or extracted. However, in the case of $\mathrm{CB}$, there was no space between the steam humidifier and the heating coil to install separate sensors. Thus, only one temperature and relative humidity sensors was previously installed by the Facilities Management and Planning (FMP) before the supply fan to determine the state of the air after the coils (heating and cooling) and steam humidifier. In this case, the analysis relied on an energy balance and valve operations obtained from the BMS to determine the amount of energy injected or extracted by coils and humidifier.

The temperature of the supply and return hot glycol water were obtained from sensors located at the main heat exchanger (steam and hot water), which provides hot water to the whole building. While the chilled water supply and return temperature were obtained from sensors located at the chiller. Furthermore, in the current study, thermal losses through the pipes transporting the fluids were excluded as they are well-insulated.

From the above-mentioned challenges, the authors recommend that future buildings be constructed with another set of temperature sensors (in different locations) for outdoor, mixed, recirculated, and exhaust air. This will provide more data to be able to determine the pattern of thermal stratification in the air stream. It is also recommended to install 
temperature sensors for the supply and return fluids running through the coils (i.e. hot glycol water and chilled water). This will enable to determination of the actual performance of the coils and thermal losses through pipes. Moreover, a flow meter is required for the steam humidifier to determine the actual amount of steam used in the humidification process.

\subsection{Data Visualization}

This study applied Sankey diagrams on HVAC sub-systems to visualize energy and mass flows and the corresponding direct or indirect energy cost through each component and stage in the system. The Sankey diagrams covered data for four months in winter (January-April 2015) and summer (May-September 2015). The proposed Sankey diagrams also show a comparison between the baseline and the actual energy consumption by different components (i.e. sensed and metered components such as fans, pumps, steam, chillers, and cooling tower fans). The baseline energy consumption of different components for the same year selected in the study was provided by the Facilities Management and Planning (FMP) on campus. The baseline energy consumption is calculated based on previous year bills. The energy flows in the following Sankey diagrams were colored to indicate the actual energy consumption compared to the baseline. Green, yellow, and red color indicates the energy consumption that are higher, equal to, and lower than baseline, respectively.

The Sankey diagrams were rendered in a browser using Scalable Vector Graphics (SVG). D3's Sankey layout code developed by Google Developers was used to create Sankey diagrams. This layout helped in providing more flexibility in organizing nodes, colors, and font sizes [183]. In order to create Sankey diagrams, which mainly consists of 
nodes with the connection links, a comma-separated values (CSV) file should be prepared at first. This CSV file consist of a set of rows containing data for source, target, and magnitude for each flow. Unfortunately, this software prevented the creation of feedback loops in the Sankey diagrams, so these were manually added with image editing software.

Sankey diagrams should be read based on the direction of the flow (i.e. from left to right). All energy inputs come from the left side, while energy outputs leave rightward. For instance, during winter, heat is added to the building. While during summer, heat is extracted from the building. This study also accounts for the amount of recovered energy, which is represented by loops that emerge from the right and re-inter the system from the left.

\subsubsection{Energy Consumption}

The purpose of the energy Sankey diagrams is to show the energy flow from the source and its distribution to different components in the HVAC system. During the winter season, the CHP generates steam, which is delivered to the building. Some of the supplied energy to the CHP is converted into useful energy (i.e. steam production). Some heat losses were recorded due to the inefficiency of the plant and the distribution losses through the pipes from the plant to the building. During the summer season, electric energy is used by the chillers and cooling towers to extract energy from the building to the ambient air. The amount of energy gained and lost were estimated using energy balance equations developed in Section 5.3 and historical data from BMS. On the other hand, enthalpy of air at $0^{\circ} \mathrm{C}$ and $0 \% \mathrm{RH}$ was selected as the reference state.

All forms of energy (i.e. electricity and steam) in this diagram were converted to 
one common energy unit: MWh. HVAC energy flows for the heating and cooling seasons are shown in Figure 5.7 and Figure 5.8, respectively. It should also be noted that the return air from the plenum is not shown in the Sankey diagram during summer season as it is the reference point used to calculate cooling loads.

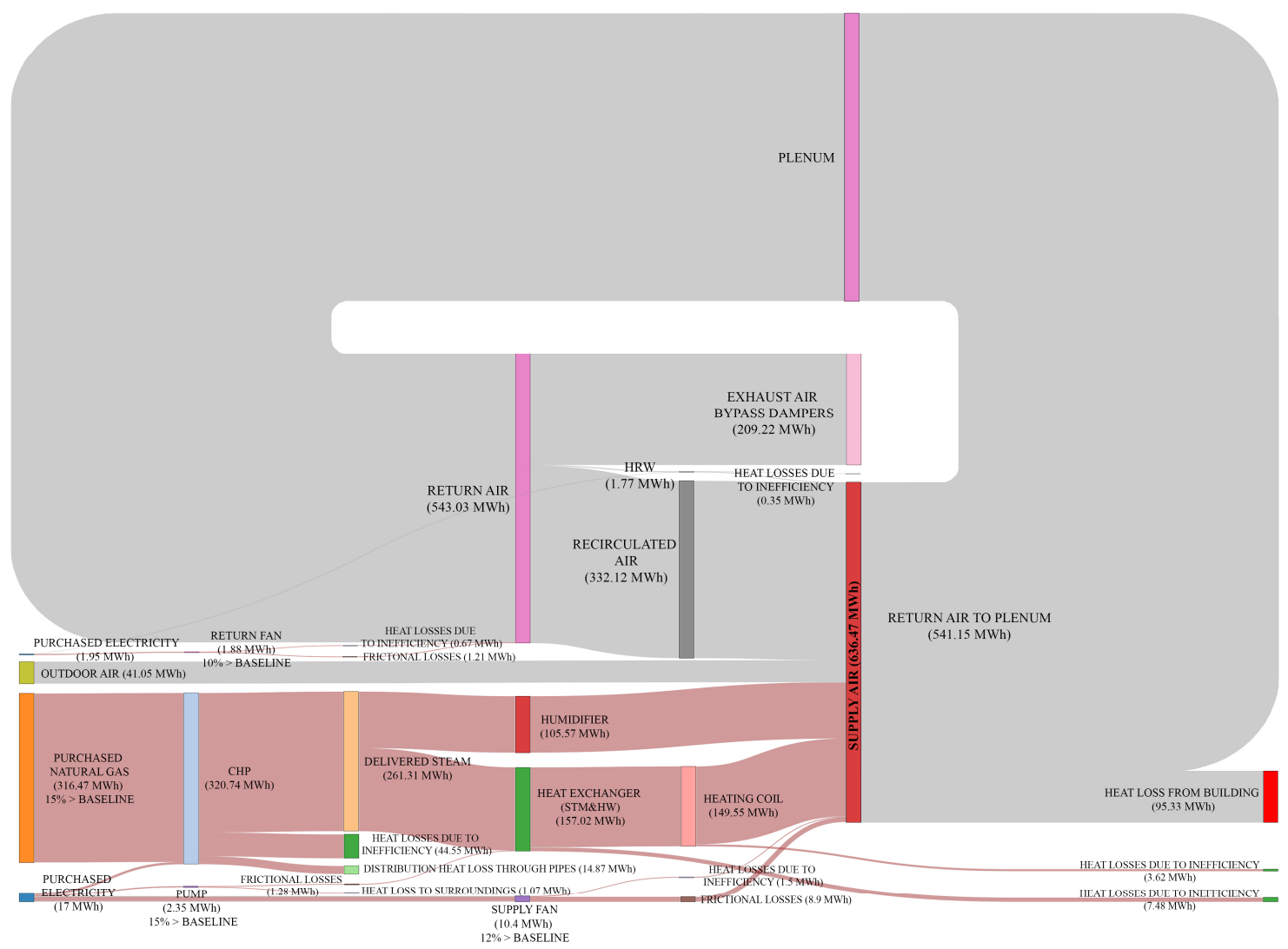

Figure 5.7: Sankey diagram showing AHU and plant loops energy flow during winter season, energy flows (for sensed and metered components) colored in red color indicates that the actual energy consumption is higher than the baseline 


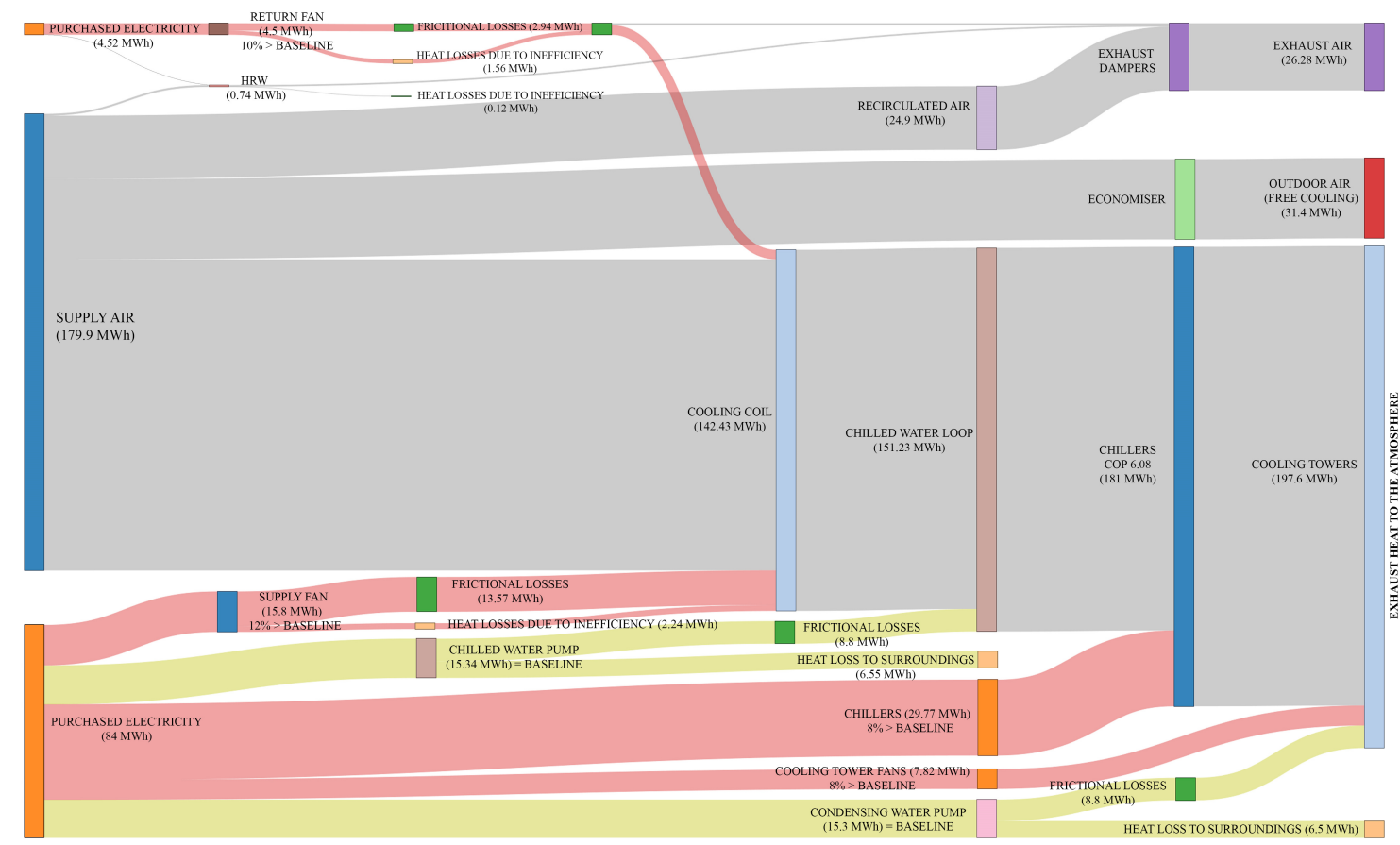

Figure 5.8: Sankey diagram showing AHU and plant loops energy flow during summer season, energy flows (for sensed and metered components) colored in red color or yellow indicates that the actual energy consumption is higher than or equal to the baseline, respectively

\subsubsection{Mass Flow}

The aim of this diagram is to show mass flow of fluids throughout the AHU and plant loops. During the winter, the diagram shows the total mass flow of steam, natural gas and their distribution through various paths. Moreover, it shows mass production of steam by the CHP and the amount delivered to the building including return condensate water. Moreover, steam losses due to inefficiency of CHP and distribution through network of pipes were also taken into consideration as shown in Figure 5.9. While in the summer, the diagram shows the chilled and condensing water loops as shown in Figure 5.10. The common unit used to represent mass flow is tonnes. Moreover, seasonal average mass flow rate in $\mathrm{kg} / \mathrm{s}$ was also included in the diagram in order to provide more understandable values. BMS provides volumetric flow rates, thus density of fluids was used to obtain mass 
flow rates. A psychrometric chart and formulas (mentioned in Chapter 1 of ASHRAE Handbook — Fundamentals (SI), 2013 [239]) were used (as the density of air is a function of temperature and relative humidity). Moreover, the density of other fluids (i.e. steam, hot water, and condensing and chilled water) was calculated based on the thermos-physical properties of the fluid (i.e. temperature, pressure, and properties of the fluid mixture). The BMS does not provide flow rate across each component, thus the fluid flow rates were obtained from the equations mentioned in Section 5.3.

On the other hand, the amount of net exfiltration and internal water vapor added were calculated as shown in Equation (5.31) and Equation (5.32), respectively.

$$
\begin{gathered}
\dot{m}_{\text {net exfiltration }}=\dot{m}_{\text {supply air }}-\dot{m}_{\text {return air }} \\
\dot{m}_{\text {internal water vapor added }}=\dot{m}_{\text {return air }} \times\left(W_{\text {return air }}-W_{\text {supply air }}\right)
\end{gathered}
$$




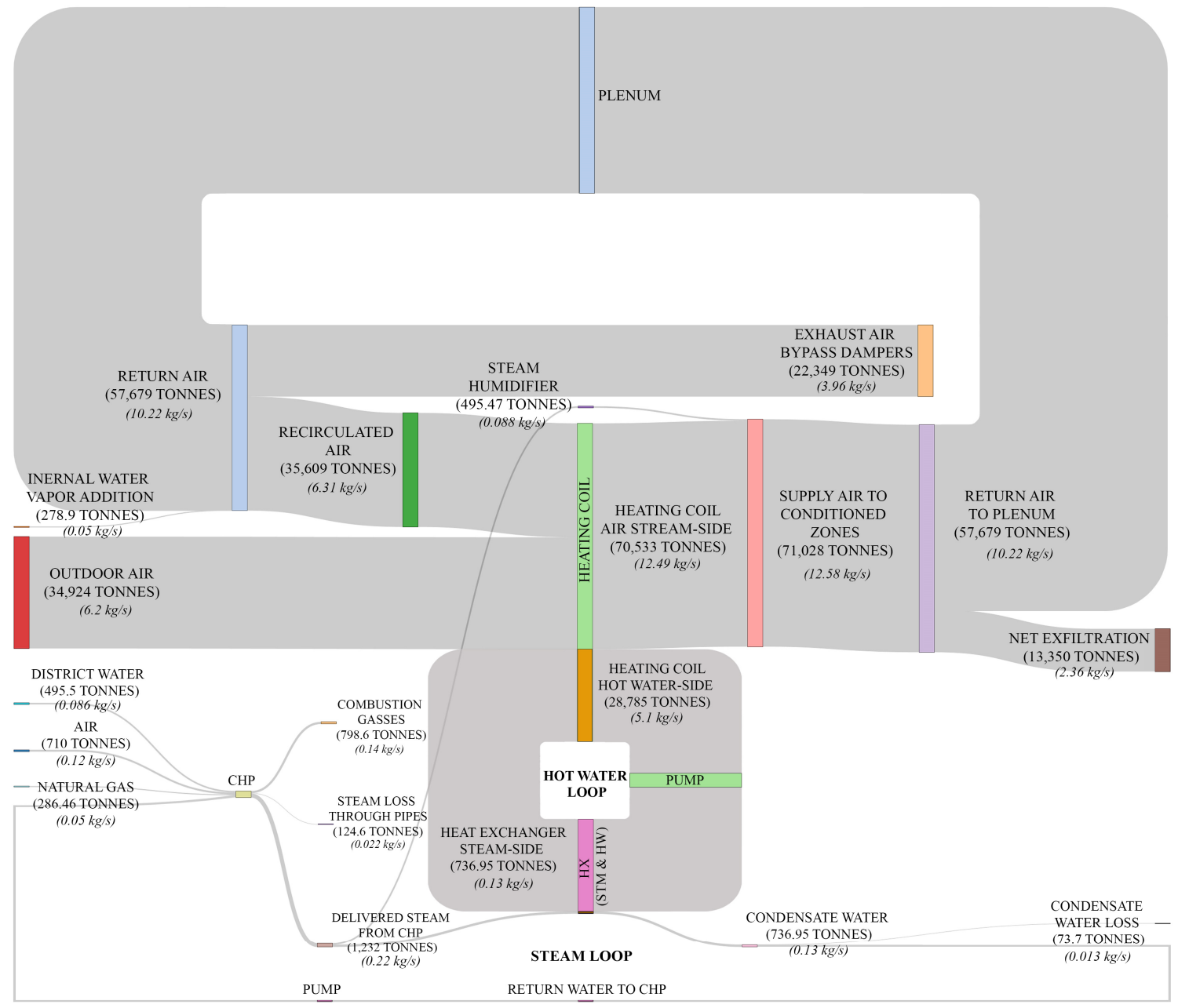

Figure 5.9: Sankey diagram showing AHU and plant loops mass flow during winter season 


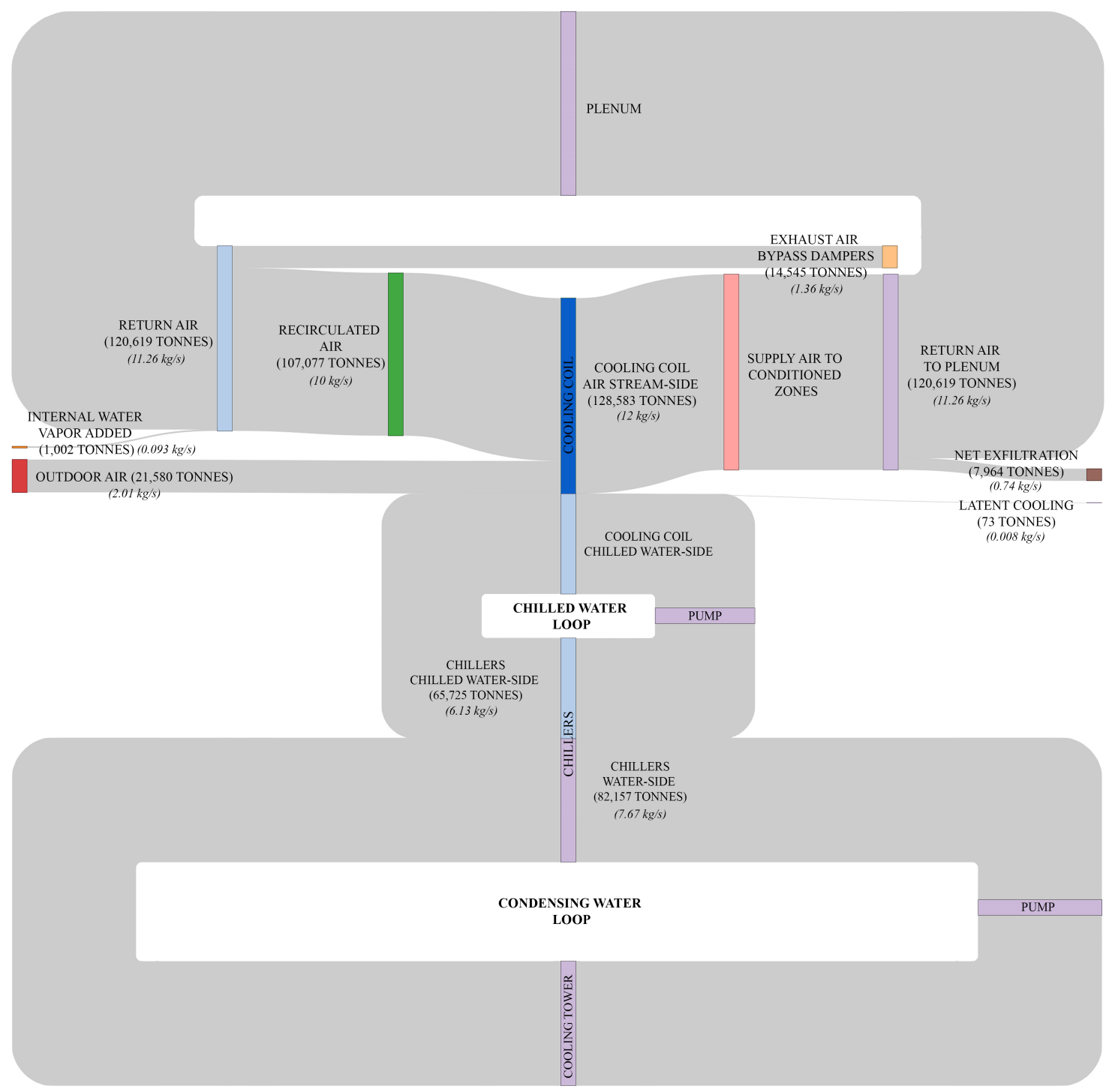

Figure 5.10: Sankey diagram showing AHU and plant loops mass flow during summer season

\subsubsection{Energy Cost}

This diagram focuses on visualizing the corresponding energy cost by different components in the AHU and plant loops. The cost of the steam was determined based on the amount of natural gas and electricity consumption by the CHP [197]. Moreover, the equivalent indirect cost of passive heat gains (i.e. from outdoor air and recirculated air) or losses (i.e. exhaust air) was also calculated by using a proportional weighting to map these 
costs to real utility costs. The enthalpy of air at $0^{\circ} \mathrm{C}$ and $0 \% \mathrm{RH}$ was used as reference point for the energy cost Sankey diagrams. However, Sankey diagrams implies that if outdoor air is $10^{\circ} \mathrm{C}$ that energy flows inwards for free; but that still has costs since we have to heat it with steam. Furthermore, the electrical to thermal cost ratio was also considered during heating season to determine the value of heating cost that is offset by heat generated by supply and return fans and the hot water pump. During summer days, the energy cost was calculated based on the coefficient of performance (COP) of the cooling system to determine the value of adverse and useful repercussions on cooling cost. For the summer season energy cost diagram, the left-hand side of the diagram shows the total purchased electricity by the chillers and cooling tower fans and the indirect cost of free cooling and exhaust air. While, on the right-hand side of the diagram, the cost to remove the corresponding heat from pumps and air handling unit fans is shown. In contrast to the Sankey diagrams representing energy, there is not necessarily a conservation of cost across a component. Figure 5.11 and Figure 5.12 show the corresponding energy cost during heating and cooling seasons, respectively. 


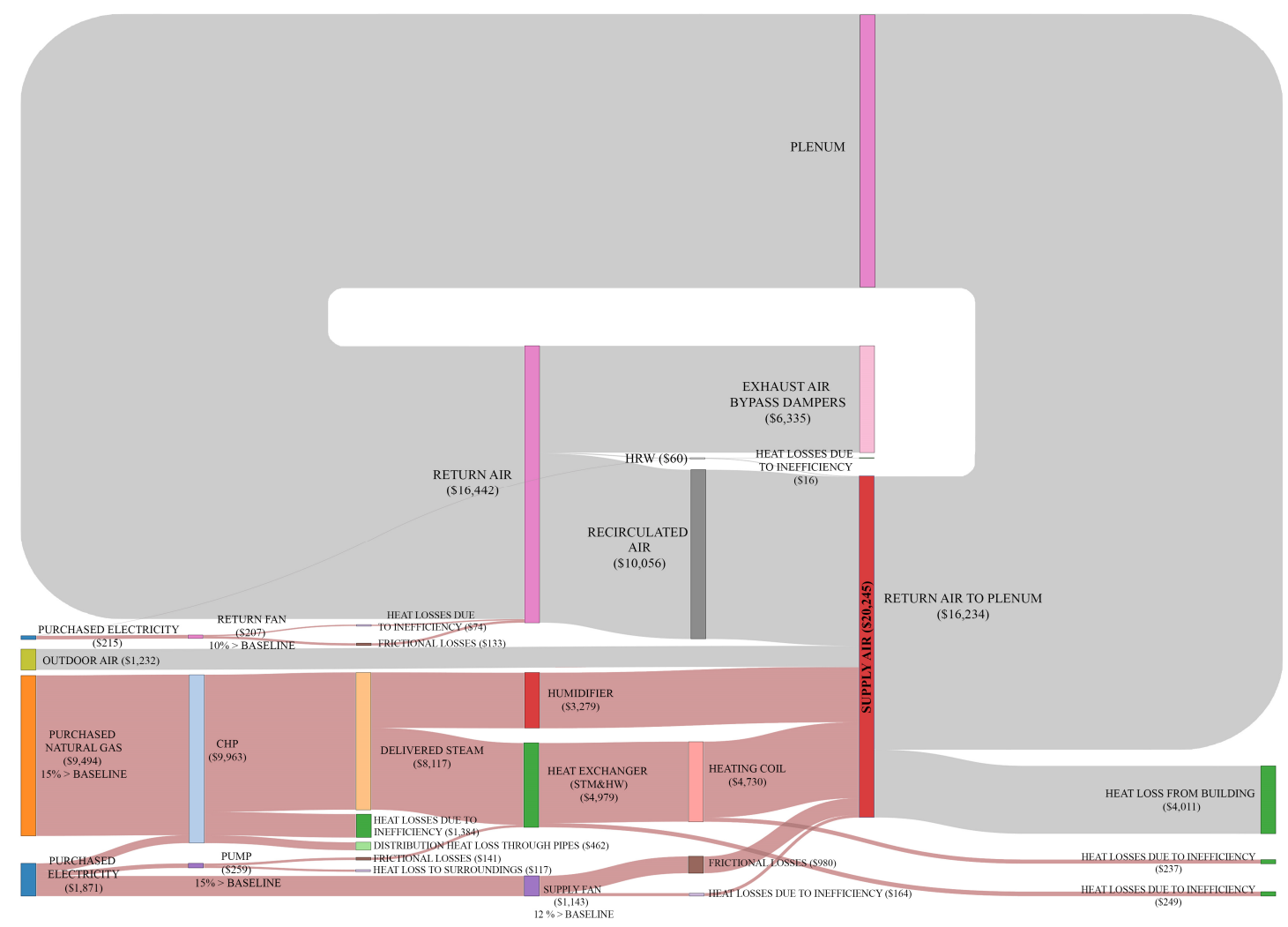

Figure 5.11: Sankey diagram showing AHU and plant loops energy cost during winter season, energy costs (for sensed and metered components) colored in red color indicates that the actual energy consumption is higher than the baseline 


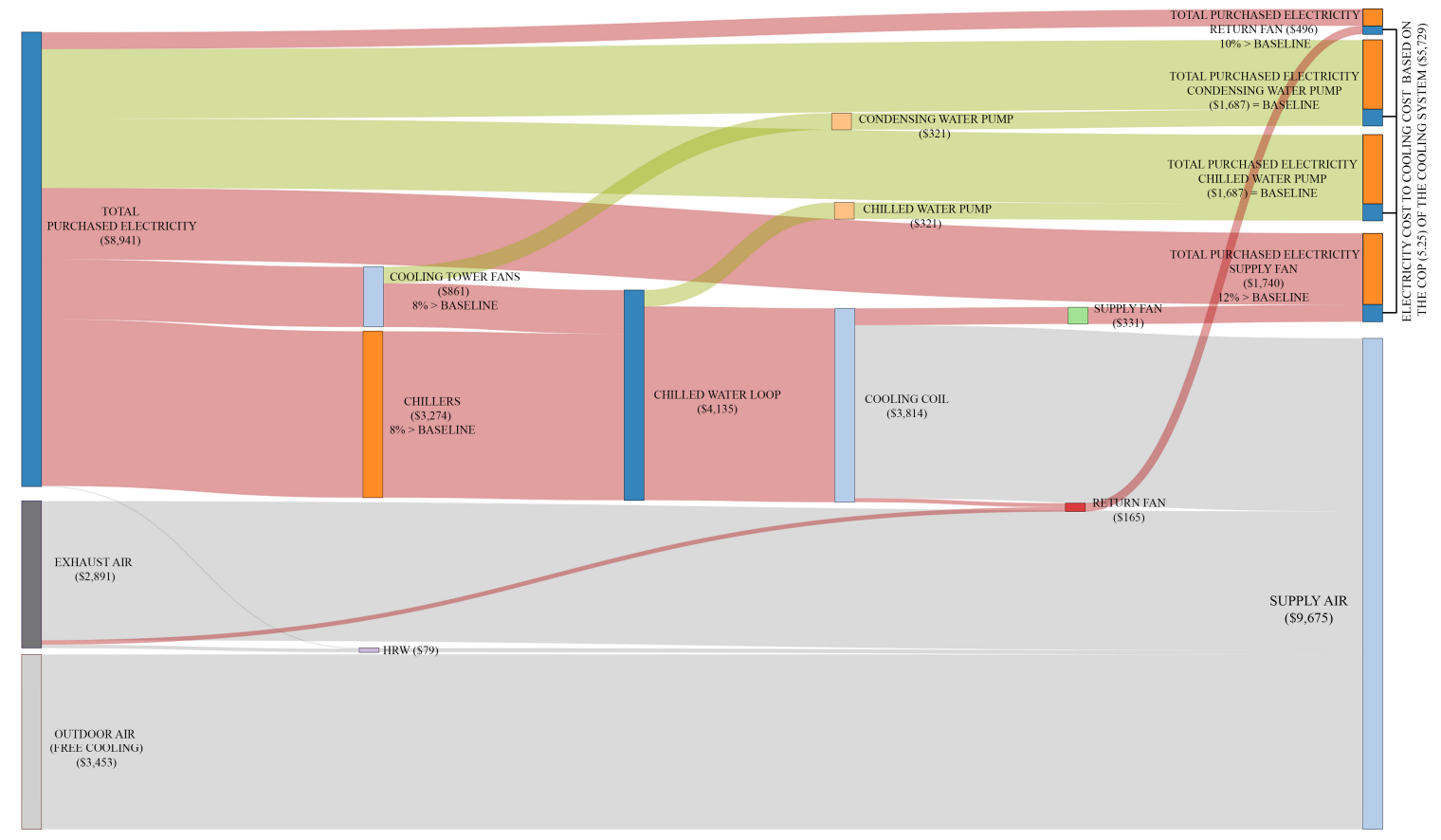

Figure 5.12: Sankey diagram showing AHU and plant loops energy cost during summer season, energy costs (for sensed and metered components) colored in red color or yellow indicates that the actual energy consumption is higher than or equal to the baseline, respectively

\subsection{Discussion}

The current study focused on sub-hourly data obtained from BMS for one AHU and plant loops during winter and summer seasons. Figure 5.7 shows the energy flows and feedback loops of AHU and plant loops during the heating season, while Figure 5.11 shows the corresponding energy cost. Approximately 23 percent $(\$ 4,730), 16$ percent $(\$ 3,279)$, 6.5 percent $(\$ 1,232)$ of the heat was added by the heating coil, steam humidifier, and outdoor air, respectively. The natural gas consumption was found to be higher than the baseline by almost 15 percent. This was at least partially due to the fact that the set point temperature was set to $22^{\circ} \mathrm{C}$, regardless of occupancy. Moreover, this building has opposite pairs of large sliding doors at the entrance, which caused significant air flow through the lobby. It was also noticed that the windows were left opened in the winter for some spaces. 
The boiler efficiency was found to be approximately 80 percent. On the other hand, the amount of energy added by outdoor air was estimated based on enthalpy at $0^{\circ} \mathrm{C}$ as reference. The majority $\sim 52$ percent $(\$ 10,056)$ of the heat was added from the recirculated air and HRW as shown in Figure 5.7. This was due to high internal gains from equipment, lighting, occupants, and heat added by VAV-reheat coils and radiant panels installed in some of the perimeter zones. However, it was observed that the HRW was rarely used. By checking the HRW controller, it was noticed that the allowable HRW operation temperature range (i.e. difference between sensed and setpoint temperature of the supply air) was very low. For instance, the allowable HRW operation temperature was found to be approximately $0.7^{\circ} \mathrm{C}$. It was also noticed that the HRW was only working when the heating system was turned off. This caused the HRW to operate only for 22 hours during winter season. On the other hand, it was recorded that the supply fan energy consumption was higher than the baseline by approximately 12 percent. The amount of heat added to the air stream by the fan was approximately 2.5 percent $(\$ 1,143)$ of the total heat supplied to the conditioned spaces. While, it was found that the return fan energy consumption was higher than the baseline by 10 percent. It was also noticed that approximately 38 percent $(\$ 6,335)$ of the return air was exhausted to the atmosphere. This might be due to high $\mathrm{CO}_{2}$ concentration of the return air, which cannot be recirculated. However, if the HRW was used more frequently, it could have recovered approximately 30 percent of the energy exhausted to the environment. This could be done by increasing the allowable HRW operation temperature range taking in consideration freezing problems that could damage the HRW. The hot glycol pump energy consumption was found to be higher than the baseline by 15 percent. Moreover, the measured efficiency of the pumps was found to be 
approximately 45 percent, while the rated efficiency is 70 percent.

Figure 5.8 shows the energy flows and feedback loops of AHU and plant loops during the cooling season. While, Figure 5.12 shows the corresponding energy cost. Approximately 67 percent $(\$ 16,215), 18$ percent $(\$ 3,453)$, and 15 percent $(\$ 2,737)$ of the heat was extracted by the cooling coil, free cooling (i.e., cooler outdoor air instead of mechanical cooling when the outdoor temperature is below $22^{\circ} \mathrm{C}$ ), and recirculated air and HRW, respectively. However, it was again observed that the HRW did not contribute significantly to recovering energy as it was rarely turned on (5 hours during the summer season) as discussed above. Moreover, the HRW was operating when the cooling coil was turned off. Approximately 20 percent of the energy could be recovered if the HRW operation temperature range increased. The supply fan energy introduced from the motor was approximately 10 percent $(\$ 1,740)$ of the total energy extracted by the cooling coils. Moreover, the supply fan energy consumption was approximately 12 percent higher than baseline. This appears to be due to high frictional losses recorded through the air filter installed before the heating and cooling coils and also the pressure losses through the ducts. In terms of temperature, it was observed that the supply fan increased the air stream temperature from $11^{\circ} \mathrm{C}$ to $15.5^{\circ} \mathrm{C}$, while the mixed air temperature was about $22^{\circ} \mathrm{C}$. While, the amount of heat addition from the return fan was approximately 3 percent of the total amount of energy extracted by the cooling coil. It was also found that the return fan energy consumption was higher than the baseline by 10 percent. The chiller energy consumption was found to be higher than the baseline by approximately 8 percent. This was due to the high internal gains from occupants, lighting, equipment, and heat gain from building envelope. Furthermore, while the coefficient of performance (COP) of the chiller is 
approximately six, the one units of electricity is required to remove 3.9 units of heat from the building. The pumps (i.e. condensing and chilled water) energy consumption were found to be the same as baseline. However, the efficiency of the pumps was found to be approximately 43 percent, while the rated efficiency is 77 percent. For the cooling tower, it was noticed that the fan's energy consumption was higher than baseline by almost 8 percent.

On the mass flow side, Figure 5.9 shows AHU and plant loops total mass flow for the whole winter season. The proposed Sankey diagrams also shows seasonal average mass flow rate that fluctuates over time. The mixed air consisted of approximately 51 percent and 49 percent from recirculated air and outdoor air, respectively. The steam humidifier added on average for the winter season approximately $0.088 \mathrm{~kg} / \mathrm{s}$ of water vapor to the air stream. Moreover, the net exfiltration was about 18 percent of the supply air, which was obtained from the difference between the mass flow of the supply and return air. Approximately $0.05 \mathrm{~kg} / \mathrm{s}$ of water vapor was added on average from occupants, gas appliances, hot water consumed by sinks, seasonal storage of moisture, construction materials, basements, and rain penetration. The average moisture addition from a sitting person is approximately $9 \mathrm{mg} / \mathrm{s}(\sim 0.75 \mathrm{~kg} /$ day) [240]. The mass flow of hot glycol-water was almost 40 percent of the mass flow of air. While, the mass flow of the steam was approximately 2.5 percent of the hot glycol-water mass flow.

Figure 5.10 shows AHU and plant loops total mass flow for the whole summer season. The mixed air consisted of approximately 83 percent from recirculated air and 17 percent outdoor air. The outdoor air fraction recorded low value as the AHU exhaust almost 12 percent of the return air. Moreover, the net exfiltration was recorded as approximately 
6.5 percent of the supply air. Approximately $0.093 \mathrm{~kg} / \mathrm{s}$ of water vapor was added on average from the internal components of the building. Furthermore, the mass flow rate of water vapor in the air stream was reduced by $0.008 \mathrm{~kg} / \mathrm{s}$ (on average) due to latent cooling. The mass flow of the chilled water was found to be less than the mass flow of air and condensing water by 51 and 75 percent, respectively.

The proposed Sankey diagrams were able to show relative energy, mass, and cost in order to identify components that deserve the most focus for improvement. Moreover, Sankey diagrams allowed to understand the performance of the whole system - not just component. It also helped in understanding the upstream and downstream impact of inefficiencies. Furthermore, Sankey diagrams facilitated the identification of possible opportunities to recover energy. The implications of showing total flow for the whole season rather than instantaneous or other shorter periods may reduce the ability to detect issues and inefficiencies.

\subsection{Summary and Future Work}

This study proposed a method to analyze and visualize the performance of real HVAC system. The study at first focused on developing a method using first principles to convert sparse HVAC data obtained from sensors and sub-meters into energy, mass, and cost flows. The estimated flows were then visualized using Sankey diagrams. The proposed method was then applied to one of the AHUs of a multi-zone Canadian university building. The main contribution of this study is to make use of real-time and historical data obtained from BMS instead of relying on simulations and predictions to identify inefficiencies. The implication of this work is that it will help make operational problems more visible and quantifiable in order to identify opportunities for energy savings and to facilitate the 
decision making by building operators to manage the operation of building system level. The proposed method facilitated greater understanding on how each component in the AHU and plant loops affect the performance of the whole system. It also helped in understanding the upstream and downstream impact of inefficiencies. Moreover, the proposed Sankey diagrams helped in comparing the energy consumption of metered and sensed components to the baseline to help non-expert users to assess these values.

There are some limitations in the usability of the proposed Sankey diagrams. For instance, they primarily provide information on the efficiency of components but do not directly identify the cause of poor operations.

Future steps for this research initiative include:

- Developing tool(s) for automating the process that creates Sankey diagrams from sensors and sub-meters' input files;

- Creating Sankey diagrams that can represent retrofitting of some AHU and plant loop components and their impact on the overall energy consumption;

- More analysis on the secondary air handling unit (e.g., VAV boxes);

- Developing interactive Sankey diagrams by allowing the user to select the temporal resolution (discussed in Chapter 7). 
Part II: Implementation of Sankey Diagrams as A DecisionMaking Tool 


\section{Chapter 6: Visualization of Building Performance using Sankey Diagrams to Enhance the Decision-Making Process}

This chapter was published as:

(Abdelalim A., O’Brien W. and Shi Z., "Visualization of Building Performance using Sankey Diagrams to Enhance the Decision-Making Process, "Symposium on Simulation for Architecture and Urban Design (SimAUD) Conference)

It is reprinted here under the terms of the copyright license agreement with ACM Digital Library. The copyright license agreement is provided in Appendix H.

This chapter aim to investigate the feasibility of utilizing Sankey diagrams in visualizing building performance and to understand the upstream and downstream impact to allow various design variants to be evaluated and facilitate the decision-making by architects and design engineers.

\subsection{Abstract}

Nowadays, there are various building energy performance optimization methods available to designers. The aim of these methods is to vary building parameters to optimize the energy performance of the building in the early design stage and during operation and to choose the appropriate alternatives evaluated through multi-criteria objectives. However, current visualization methods have some limitations in evaluating simulation results in relation to non-performative or qualitative analysis. This paper investigates the feasibility of using Sankey diagrams to visualize and understand the upstream and downstream performance impacts of building design decisions. The current target audience is primarily architects and design engineers. The aim of this paper is to provide a workflow to obtain, analyze, and visualize energy flows obtained from simulation outputs. The 
developed workflow is applied to large office commercial reference building models that comply with the national energy code of Canada for buildings. Samples of Sankey diagrams are presented to visualize the impact of changing building/system components on the whole system performance and demonstrate energy-saving strategies.

\subsection{Introduction}

Energy demand in commercial and intuitional buildings accounts for 16 percent and 14 percent of the total energy consumption in U.S. [241] and Canada [242], respectively. Optimization of building parameters (such as geometry, orientation, materials, and construction) in early design stages or components (such as mechanical, electrical, and control systems) during operation is crucial to reduce energy use and greenhouse gas emissions [243]. Integrating parametric modeling into the process of energy performance analysis helps designers evaluate different alternatives through complex multi-criteria objectives [244].

On the visualization side, the most common visualization techniques used for parameter design of buildings are interactive parallel coordinates plot, binning plots, carpet plots, voxel-plot, superimposed line graph plot, line chart, bar graph, and color mapping plots. Jeong et al. [245] developed an integrated environment combining BIM and the results from object-based building energy simulation (BES). Their method helped in visualizing energy simulation results of each building component one at a time in BIM environment. Moreover, objects such as infiltration, ventilation, solar radiation, and occupants were not included in their study. Asl et al. [114] developed an integrated parametric BIM-based system to interact with cloud-based whole building simulation and daylight tools to optimize the building energy performance using a multi-objective 
optimization. Their system helped designers to explore different design alternatives using a visual programming interface and to assess the energy performance to select the most appropriate window design. Interactive parallel coordinates plot was used in their study to visualize various iterations to be evaluated by users. Raftery and Keane [116] presented a new visualization technique of building performance data by combining binning with carpet plots. Pratt and Bosworth [10] developed a multi-scalar visualization technique that could help users understand the effect of changing building parameters on energy performance. Three-dimensional interactive voxel-plot and superimposed line graph plot were used in their study to visualize the output simulation results. Pratt and Bosworth claimed that an effective visualization method is needed when inspecting a high order (more than three variables) search space.

Moreover, Pratt and Bosworth [10], Srivastav et al. [11], and Hab et al. [12] claimed that conveying simulation results in the form of tables and graphs is not useful to nonexpert designers in the field of building science. Elnimeiri and Nicknam [246] visualized the results of environmental and structural performance using color mapping, which enabled the design team to understand the effect of different designs. Currently, Autodesk developed Insight $360^{\circ}$ tool [247], which empowers users to evaluate different design scenarios that could lead to a better building performance. The above-mentioned visualization methods have some limitations in evaluating simulation results in relation to non-performative or qualitative analysis [10]. Moreover, the current visualization tools typically do not provide a comprehensive understanding of how each component affects the whole system performance, including upstream and downstream building systems.

Sankey diagrams are one of the visualization tools that are useful in energy 
management and performance improvement. These diagrams consist of arrows that represent the magnitude and direction of the flow (i.e. energy flow) from source to sink [197]. Furthermore, Sankey diagrams can provide relative magnitude of the flow, input and output of interacting systems, energy recovery, and spatial representation. There are various applications of using Sankey diagrams in visualizing energy flows. On the campus level, Sankey diagrams were utilized to visualize energy, mass, greenhouse gases, and utility cost in a companion paper by Abdelalim et al. [197]. In another companion paper, Sankey diagrams were utilized to visualize energy flows and associated costs on the building-level by using measured and calibrated model data [248]. Belzer [198] used Sankey diagrams to visualize energy flows from source to end-use in the building sector. Dynamic Sankey diagrams were also used to visualize internal and external flows through building envelopes [199]. Schlueter and Thesseling [8] integrated Sankey diagrams into building information modeling (BIM) to visualize instantaneous energy and exergy. Sankey diagrams were also integrated to limited number of building design and analysis tools to visualize predicted energy use, such as CASAnova [154] and Sefaira [155]. On the HVAC system level, Sankey diagrams were utilized to visualize real HVAC performance of a large commercial building [249]. Sankey diagrams were also used in the work by Perez-Lombard et al. [220] to visualize energy flows in a typical constant air volume systems installed in office buildings in Spain. The current applications/tools utilizing Sankey diagrams to visualize building-level energy performance used set of nodes to represent inputs and outputs of energy at the building rather than multi-staged nodes. Moreover, the current tools have some limitations in comparing multiple designs simultaneously. 
Recently, an online survey was conducted to assess usability and effectiveness of visualizing energy flow and the associated cost using Sankey diagrams on the buildinglevel. The survey was applied to a sample user group (42 participants). The sample group consisted of 13 energy managers, 12 design engineers, 6 researchers/lecturers, and 11 other related professionals. 78 percent of the participants supported the notion that Sankey diagrams helped in: 1) visualizing building-level energy flows and costs, 2) understanding the interrelated variables that affect building performance, 3) identifying system inefficiencies, 4) quantifying and understanding the impact of unmeasured energy flows, and 5) making operational problems more visible and quantifiable in order to identify opportunities for energy savings and facilitate decision making.

This paper investigates the feasibility of utilizing Sankey diagrams in visualizing building energy performance and to understand the upstream and downstream impact to allow various design variants to be evaluated and facilitate the decision-making by architects and design engineers. The target audience is often policy makers and other nontechnical professionals without a building physics background. Thus, communicating relative energy flows is important to provide them with a basis for informed decisionmaking.

This paper proposes a workflow to obtain, analyze, and visualize energy flows obtained from simulation outputs. The developed workflow is applied to large office reference building model that complies with the National Energy Code of Canada for Buildings (NECB) (as the base case) for Ottawa climate zone (ASHRAE Climate Zone 6) [250]. Reference building models are used for building code development, research, and design guides. Sankey diagrams are used to inspect the energy-saving opportunities for the 
base case. Different design scenarios are proposed and compared to the base case model energy performance. Samples of Sankey diagrams are presented to visualize the impact of changing building/system components on the whole system performance and demonstrate energy-saving strategies. The proposed Sankey diagrams consists of multi-staged nodes, rather than merely inputs and outputs, to provide a greater understanding of how each building component/system consumes energy and relates to the others. Moreover, the paper discusses the potential of each design option in terms of energy savings using Sankey diagrams. The paper also discusses the technical challenges of creating Sankey diagrams from simulation data.

\subsection{Methodology}

The aim of the proposed study is to utilize Sankey diagrams to visualize building energy performance on the building-level and building system level (HVAC system) obtained from simulation outputs. The paper proposes a workflow to obtain, analyze, and visualize energy flows obtained from simulation outputs as shown in Figure 6.1. EnergyPlus V8.6 was selected as the BPS tool due to its technical documentation and versatility and capability of simulating complex building systems [200]. In this paper, a large office building model that complies with the National Energy Code of Canada for Buildings (NECB) for Ottawa climate zone was used as the base case model. The workflow consists of:

1) Converting sub-hourly data into annual energy flows by selecting the appropriate output variables and meters that are listed in Section 6.3.2.

2) Aggregating energy flows: some variables, such as (windows heat gain/loss 
and transmitted solar radiations, heating coils, conduction heat gain/loss rates people sensible heating and latent energy gains, air stream nodes, zone infiltration total heat gain/loss energy, and surface heat storage loss/gain rate) provides output for each zone or element. Thus, zones or elements for each variable were summed up.

3) Checking the energy balance. This process was performed by adding energy flows entering and leaving the building. As EnergyPlus provides dynamic simulation results (i.e. not steady-state for each hour). Thus, output variables such as energy input or extraction of radiant panel, boiler, chiller, and heating and cooling coils were modified to maintain energy balance for each hour and also maintain daily and monthly energy consumption.

The following sections provide details on the selecting base case model and the required simulation output variables and meters that creates Sankey diagrams.

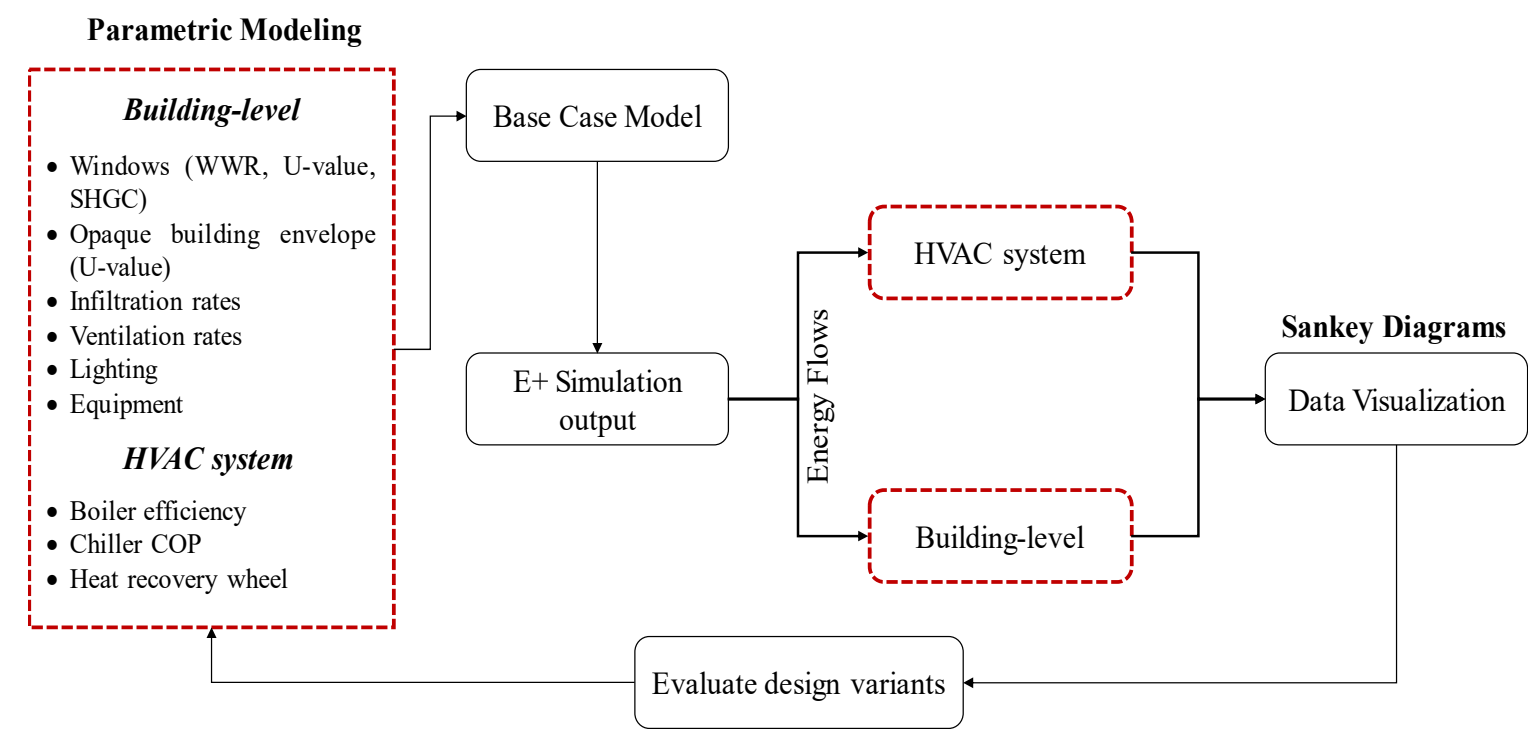

Figure 6.1: Flowchart showing the process to visualize energy flows on building-level and HVAC system using Sankey diagrams and evaluate different design variants 


\subsubsection{Setting Up the Model}

The purpose of this section is to provide information on the selected EnergyPlus V8.6 base case model. The base case model represents large office Canadian reference building provided by Natural Resources of Canada [251]. Table 6.1 shows base case model details summary. Figure 6.2 shows base case model for large office building used for Ottawa climate. AMY (actual meteorological year) weather data was used for Ottawa, Canada [202]. Schedules for internal gains used are based on American Society for Heating, Refrigerating and Air Conditioning Engineers (ASHRAE) 90.1.

Table 6.1: Base case model details summary

\begin{tabular}{|c|c|c|}
\hline \multirow{5}{*}{ General } & Bldg. type & Large office \\
\hline & Floor Area $\left(\mathrm{m}^{2}\right)$ & 14,252 \\
\hline & No. of stories (including basement) & 12 \\
\hline & $\begin{array}{l}\text { No. of thermal zones ( } 4 \text { perimeters } \\
\text { and } 1 \text { core zones/typical floor) }\end{array}$ & 56 \\
\hline & $\begin{array}{l}\text { Window to wall ratio (WWR) for } \\
\text { all orientations: }\end{array}$ & 44.57 \\
\hline \multirow{6}{*}{$\begin{array}{l}\text { Building Envelope } U \text { - } \\
\quad \text { value }\left(\mathrm{W} / \mathrm{m}^{2} . \mathrm{K}\right)\end{array}$} & Roof & 1.881 \\
\hline & Walls & 0.454 \\
\hline & Below grade walls & 3.663 \\
\hline & $\begin{array}{l}\text { Intermediate floor and basement } \\
\text { floor }\end{array}$ & 1.881 \\
\hline & $\begin{array}{l}\text { Windows (double glazed }(6 \mathrm{~mm}) \\
\text { with } 6 \mathrm{~mm} \text { air gap: clear from inside } \\
\text { and tinted from outside) }\end{array}$ & 3.045 \\
\hline & Window (SHGC) & 0.368 \\
\hline \multirow{3}{*}{ Internal Gains } & $\begin{array}{l}\text { People: office/mechanical rooms } \\
\text { (person } / \mathrm{m}^{2} \text { ) }\end{array}$ & $0.05 / 0.00495$ \\
\hline & $\begin{array}{l}\text { Lighting: office/mechanical rooms } \\
\left(\mathrm{W} / \mathrm{m}^{2}\right)\end{array}$ & $10.9 / 13.29$ \\
\hline & $\begin{array}{l}\text { Equipment: office/mechanical } \\
\text { rooms }\left(\mathrm{W} / \mathrm{m}^{2}\right)\end{array}$ & $7.5 / 1$ \\
\hline Infiltration Rates & $\begin{array}{l}\text { Floor per exterior surface area } \\
\left(\mathrm{m}^{3} / \mathrm{s}-\mathrm{m}^{2}\right)\end{array}$ & 0.00025 \\
\hline HVAC air loop & \multicolumn{2}{|c|}{$\begin{array}{l}\text { The building is conditioned by four air handling units (AHUs) } \\
\text { AHU-fan efficiency is } 55 \% \text {. No heat recovery is installed. Air }\end{array}$} \\
\hline
\end{tabular}




\begin{tabular}{|c|c|}
\hline & distribution system is single duct VAV-box \\
\hline Space Cooling & $\begin{array}{c}\text { Two electric EIR chillers with COP of } 2.5 \text { and a cooling tower } \\
\text { single speed. The building relies on free outdoor cooling when } \\
\text { the outdoor temperature is below } 28^{\circ} \mathrm{C}\end{array}$ \\
\hline Space Heating/Hot water & $\begin{array}{l}\text { Hot water delivered from a boiler using natural gas }(83 \% \\
\text { thermal efficiency). All zones are equipped with radiant panels }\end{array}$ \\
\hline Thermostat settings & $\begin{array}{l}\text { heating/cooling setpoints are } 22^{\circ} \mathrm{C} \text { to } 24^{\circ} \mathrm{C} \text {, respectively. } \\
\text { heating/cooling setbacks are } 18^{\circ} \mathrm{C} \text { to } 27^{\circ} \mathrm{C} \text {, respectively. }\end{array}$ \\
\hline
\end{tabular}

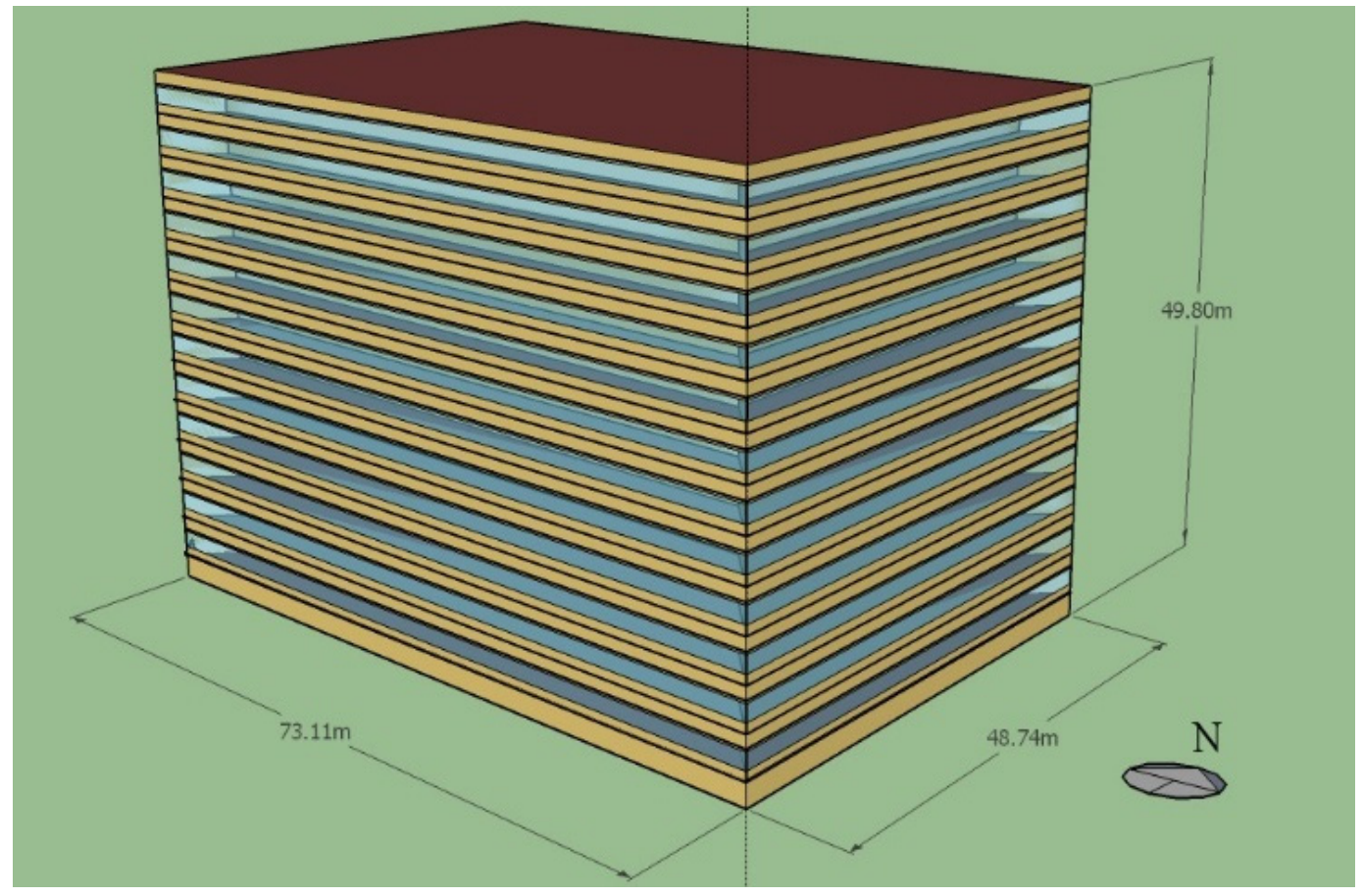

Figure 6.2: Base case model for the large office building

\subsubsection{Simulation Outputs}

Energy simulation tools provide numerous output variables and meters, from which the user has to choose the appropriate ones based on the required analysis. Table 6.2 shows the outputs that were used in this study in order to create Sankey diagrams.

Table 6.2: Hourly simulation output variables used in the study

\begin{tabular}{|c|l|}
\hline Output variable & \multicolumn{1}{c|}{ Description } \\
\hline \multirow{2}{*}{ Boiler } & Heating energy \\
& $\begin{array}{l}\text { Gas energy } \\
\text { Ancillary electrical energy }\end{array}$ \\
\hline
\end{tabular}




\begin{tabular}{|c|c|}
\hline Chillers & $\begin{array}{l}\text { Chillers electricity } \\
\text { Evaporator cooling energy } \\
\text { Condenser heat transfer rate }\end{array}$ \\
\hline Cooling tower & $\begin{array}{l}\text { Fan energy } \\
\text { Heat transfer rate }\end{array}$ \\
\hline Pump & $\begin{array}{l}\text { Pump electric energy } \\
\text { Pump fluid heat gain }\end{array}$ \\
\hline AHU & $\begin{array}{l}\text { AHU-fan energy } \\
\text { Humidifier } \\
\text { Heating coil } \\
\text { Cooling coil } \\
\text { Heat exchanger (heat recovery) } \\
\text { Outdoor air node energy } \\
\text { Exhaust air node energy } \\
\text { Return air node energy } \\
\text { Mixed air node energy }\end{array}$ \\
\hline Baseboard & Total heating energy \\
\hline People & $\begin{array}{l}\text { Sensible heating energy } \\
\text { Latent energy }\end{array}$ \\
\hline Windows & $\begin{array}{l}\text { Heat gain } \\
\text { Heat loss } \\
\text { Transmitted solar radiation }\end{array}$ \\
\hline Infiltration & $\begin{array}{l}\text { Zone infiltration total heat gain } \\
\text { Zone infiltration total heat loss }\end{array}$ \\
\hline Lighting & Electric energy \\
\hline Equipment & Electric energy \\
\hline Opaque envelope & $\begin{array}{l}\text { Outside surface heat loss } \\
\text { Outside surface heat gain }\end{array}$ \\
\hline
\end{tabular}

\subsection{Data Visualization}

This section includes samples of data visualization of energy flows on the buildinglevel and one of the air handling units (AHU) and plant loops for the base case model using Sankey diagrams for winter and summer seasons. Figure 6.3 and Figure 6.4 shows energy flows on the building-level and HVAC system for the base case (BC), respectively. The Sankey diagrams presented in this paper focused on hourly data obtained from EnergyPlus simulation outputs. Sankey diagrams should be read based on the direction of the flow (i.e. from left to right). For instance, during winter, heat is added to the building from left side and leaving rightward. While during summer, heat is extracted from the building. All forms of energy in these diagrams were converted to one common energy unit (MWh). The 
Sankey diagrams were rendered in a browser using Scalable Vector Graphics (SVG). The layout was derived from D3's Sankey layout code developed by Google Developers that provided more flexibility in organizing nodes, colors, and font sizes [183].

For the base case, the exhaust air from the AHU recorded the highest source $(\sim 60$ percent) of heat loss during winter season. This is due to the lack of heat recovery wheel (HRW) installed. Moreover, approximately 21 and 17 percent of the heat was lost through opaque envelope and windows, respectively. While, that the results show that the amount of heat was added by windows was 7 percent. This is due to the high windows USI-value. During summer season, the highest source (31 percent) of heat gain was from lighting. While, approximately 22,18 , and 17 percent of heat was added by windows, equipment, and opaque envelope, respectively. It was also found that lighting is responsible for approximately 51 percent of total internal heat gain. Moreover, the internal heat gains are responsible for 60 percent of mechanical cooling energy provided.

The next step in this study is to change some building/system components to optimize the building energy performance. Based the above analysis, windows and lighting will be modified on the building-level. While on the building system level (Heating, Ventilation, and Air Conditioning (HVAC)), a HRW will be installed to take advantage of the exhaust air in the AHU. These parameters are listed in detail in the next section. 


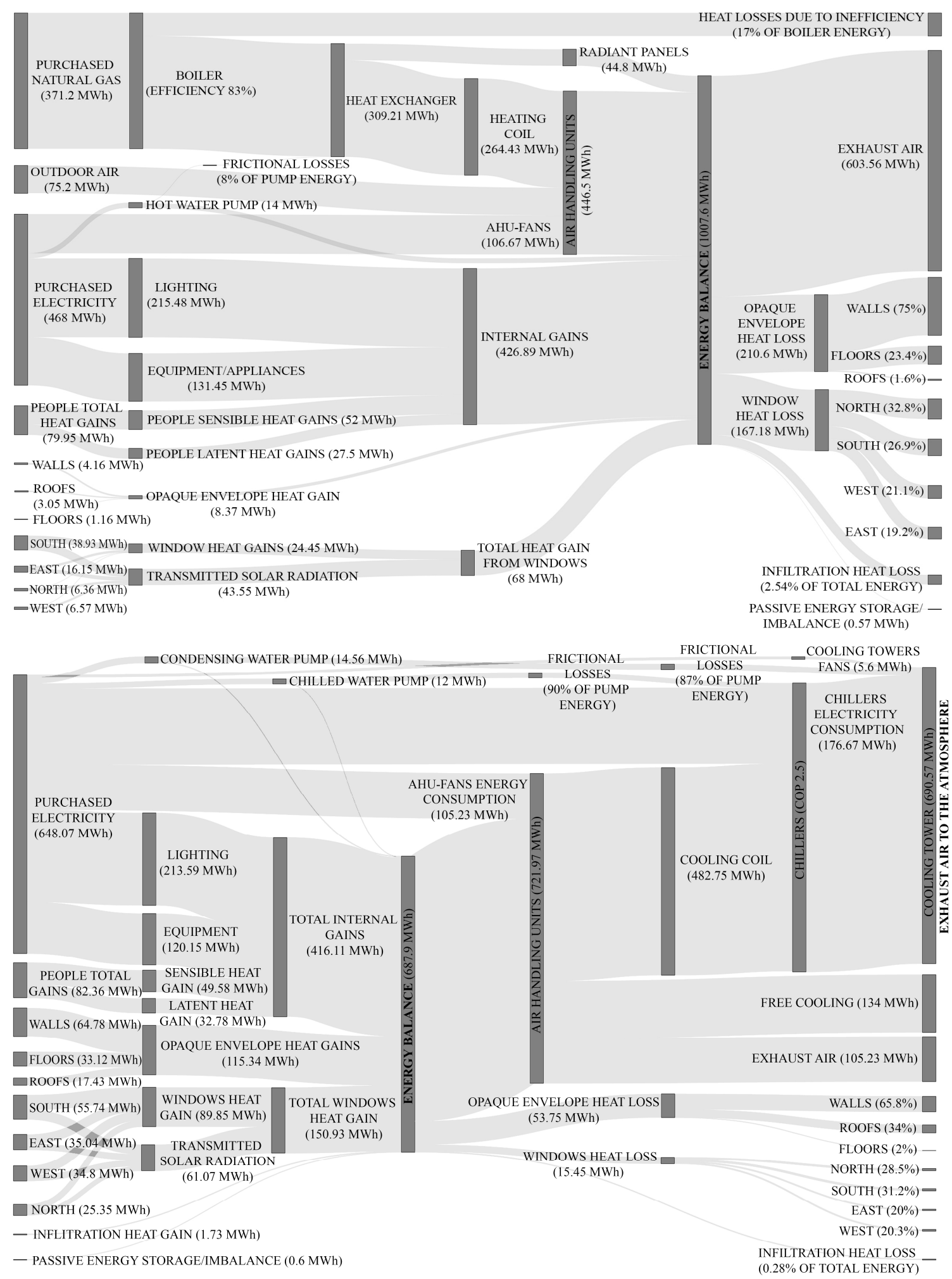

Figure 6.3: Sankey diagrams showing energy flows on the building-level for the winter season (top) and summer season (bottom) for the base case (BC) 

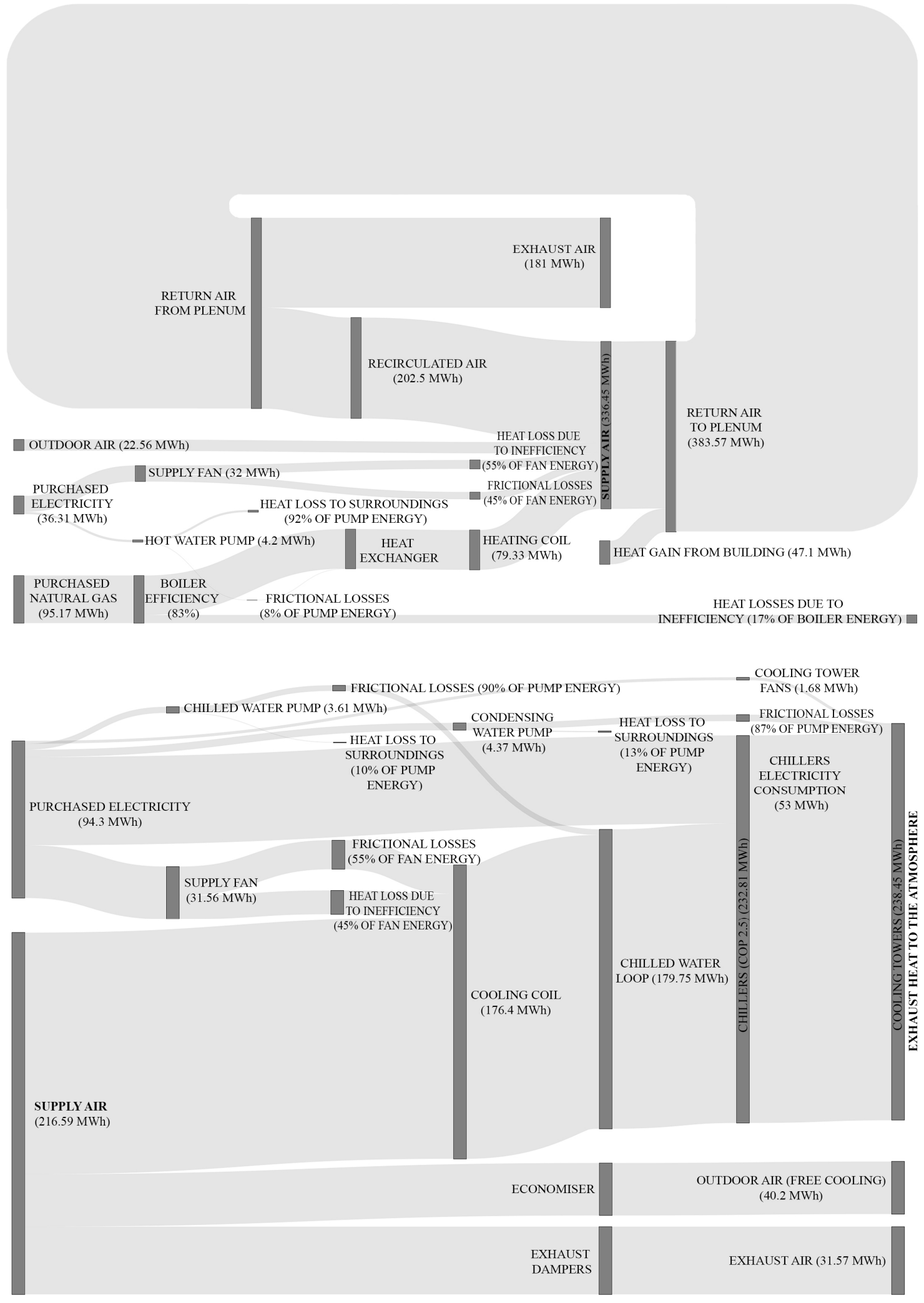

Figure 6.4: Sankey diagrams showing energy flows on the HVAC system for the winter season (top) and summer season (bottom) for the base case (BC) 


\subsection{Parametric Modeling}

There are various parameters that could be modified on the building-level and building system level to optimize the building energy performance. Table 6.3 shows different design variants implemented in this study. Each design variant was applied independently and compared to the base case.

Table 6.3: Design variants details

\begin{tabular}{|c|l|c|}
\hline Design variants & \multicolumn{1}{|c|}{ Parameters } & Level \\
\hline Base case (BC): (grey) & \multicolumn{1}{|c|}{ Details mentioned in Table 6.1} \\
\hline I1: (red) & Windows: reduce U-value to $1.903 \mathrm{~W} / \mathrm{m}^{2} \mathrm{~K}$ and & Building \\
\hline I2: (blue) & SHGC to 0.252 & Building \\
\hline I3: (green) & Install rotary heat recovery wheel (HRW) & HVAC \\
\hline
\end{tabular}

\subsection{Discussion}

The aim of this study was to investigate the feasibility of utilizing Sankey diagrams in visualizing energy flows on different spatial resolutions obtained from simulation outputs. A workflow was developed to obtain and analyze vast data sets obtained from simulation outputs and convert them to energy flows to create Sankey diagrams. Large office Canadian reference building was used as the base case. Sankey diagrams showing energy flows for base case on building-level and HVAC are shown in Figure 6.3 and Figure 
6.4, respectively. From the analysis mentioned in Section 6.4 on the base case (BC), parameters such as windows, lighting, and HRW were selected to be modified based on their significance on heating and cooling loads. The three proposed design variants are (I1, I2, and I3). Sankey diagrams were utilized to compare different design variants to the base case model energy performance. Energy flows on building-level for winter and summer seasons for I1, I2, and I3 are shown in Figure 6.5, Figure 6.7, and Figure 6.9, respectively. Figure 6.6, Figure 6.8, and Figure 6.10 shows energy flows on HVAC system for winter and summer seasons for I1, I2, and I3, respectively.

In the base case, the windows were responsible for 17 percent of heat loss during summer. While during summer, the amount of heat gain from windows was 22 percent. Thus, the first design variant (I1) aimed at decreasing windows U-value from 3.045 to $1.903\left(\mathrm{~W} / \mathrm{m}^{2} \mathrm{~K}\right)$ and SHGC from 0.368 to 0.252 . During the heating season, window heat losses and heat gains were reduced by 42 and 48.4 percent compared to the base case, respectively. As the ratio of window heat losses to heat gains was 2.45:1, energy consumption by radiant panels, AHU-heating coils, and AHU-fans were reduced by 45.7, 15.5, and 10 percent compared to the base case, respectively. This design variant (I1) resulted in a reduction of 19.8 percent of natural gas consumption by the boiler compared to the base case. During cooling season, it was found that heat gains and losses from windows were reduced by 40.6 and 49.4 percent compared to the base case, respectively. Despite the fact that window heat gains were substantially reduced, the chillers electric energy consumption was only reduced by 3.2 percent compared to the base case. Moreover, AHU-fans, chilled water pump and condensing water pump electric energy consumption were reduced by 3.8 percent. This is due the fact that window heat gains accounts for 
CHAPTER 6: VISUALIZATION OF BUILDING PERFORMANCE USING SANKEY

approximately 22 percent of the total energy gains. Moreover, the reduction recorded for the heat loss from windows caused an increase in the amount of energy on the buildinglevel that should be extracted by mechanical system. 

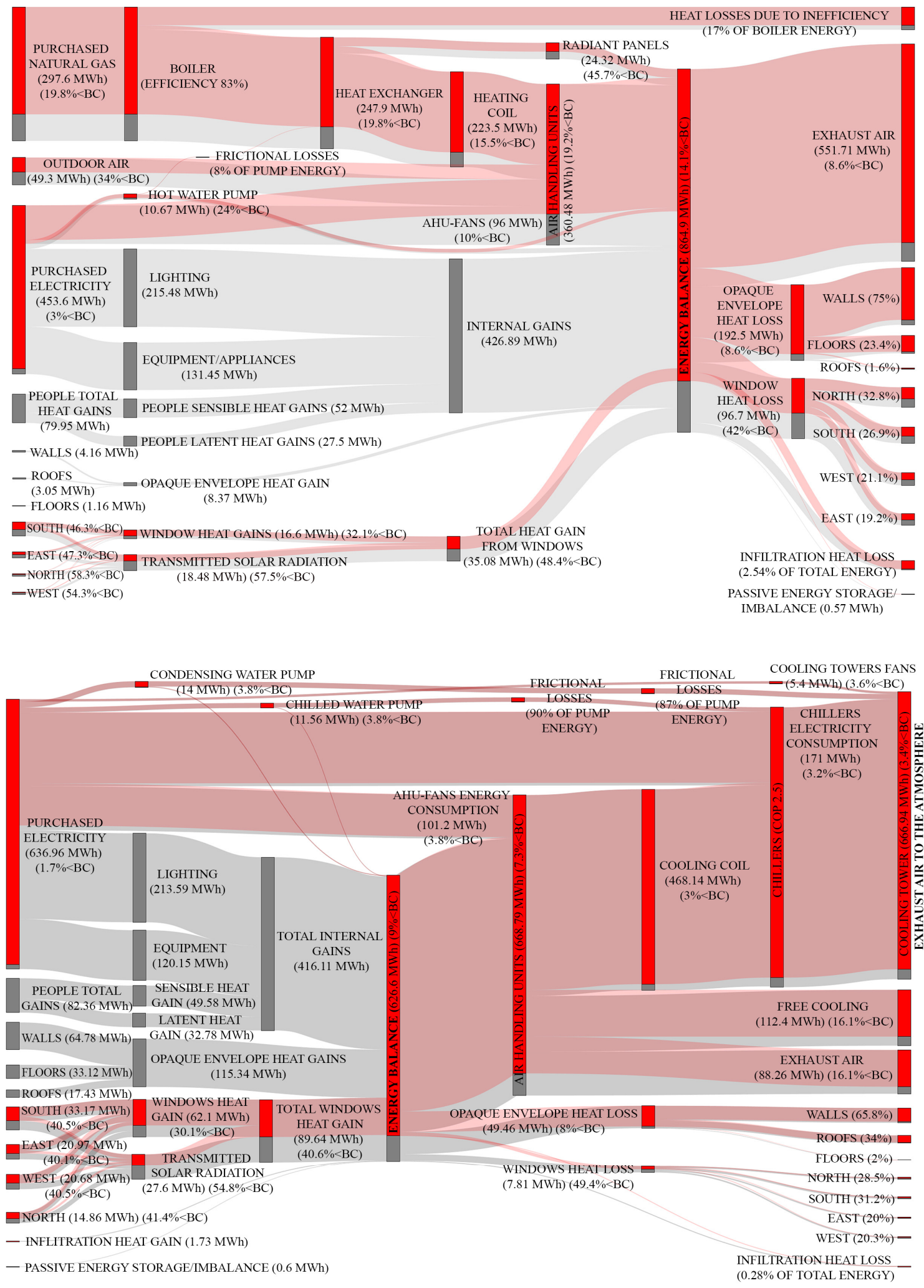

Figure 6.5: Sankey diagrams showing energy flows (grey for BC and red for I1) on building-level for the winter season (top) and summer season (bottom) 


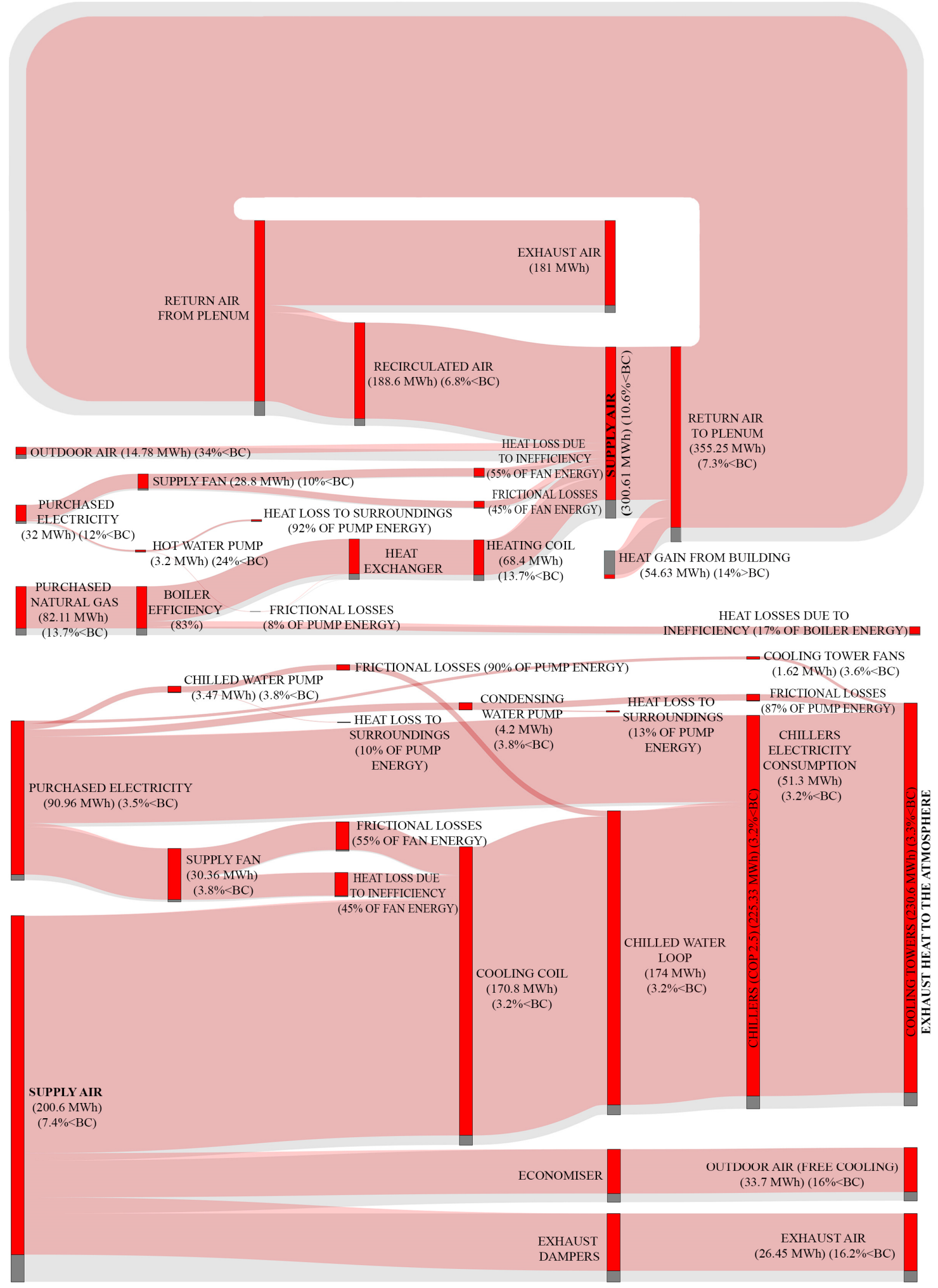

Figure 6.6: Sankey diagrams showing energy flows (grey for BC and red for I1) on HVAC for the winter season (top) and summer season (bottom) 

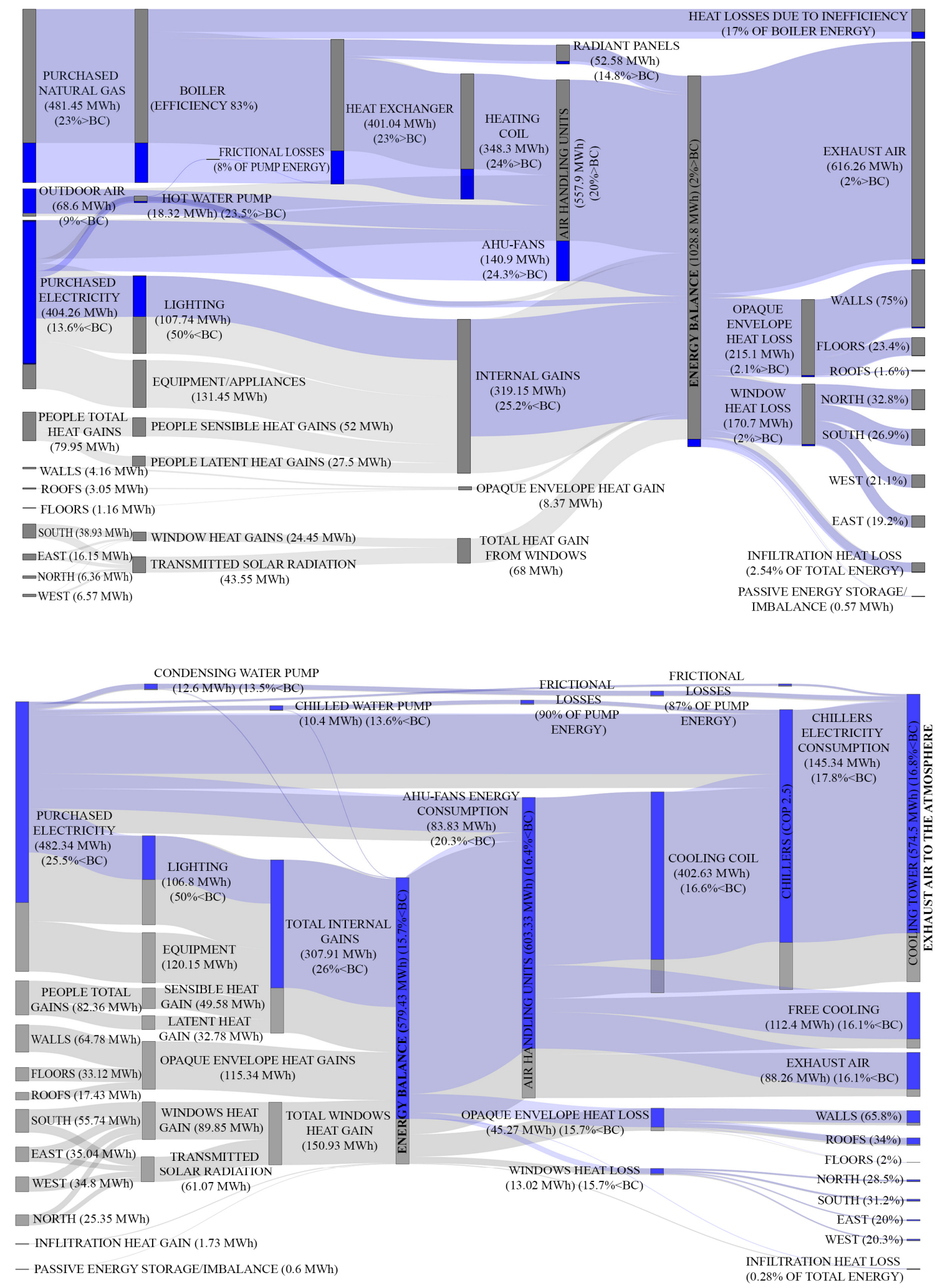

Figure 6.7: Sankey diagrams showing energy flows (grey for BC and blue for I2) on building-level for the winter season (top) and summer season (bottom) 


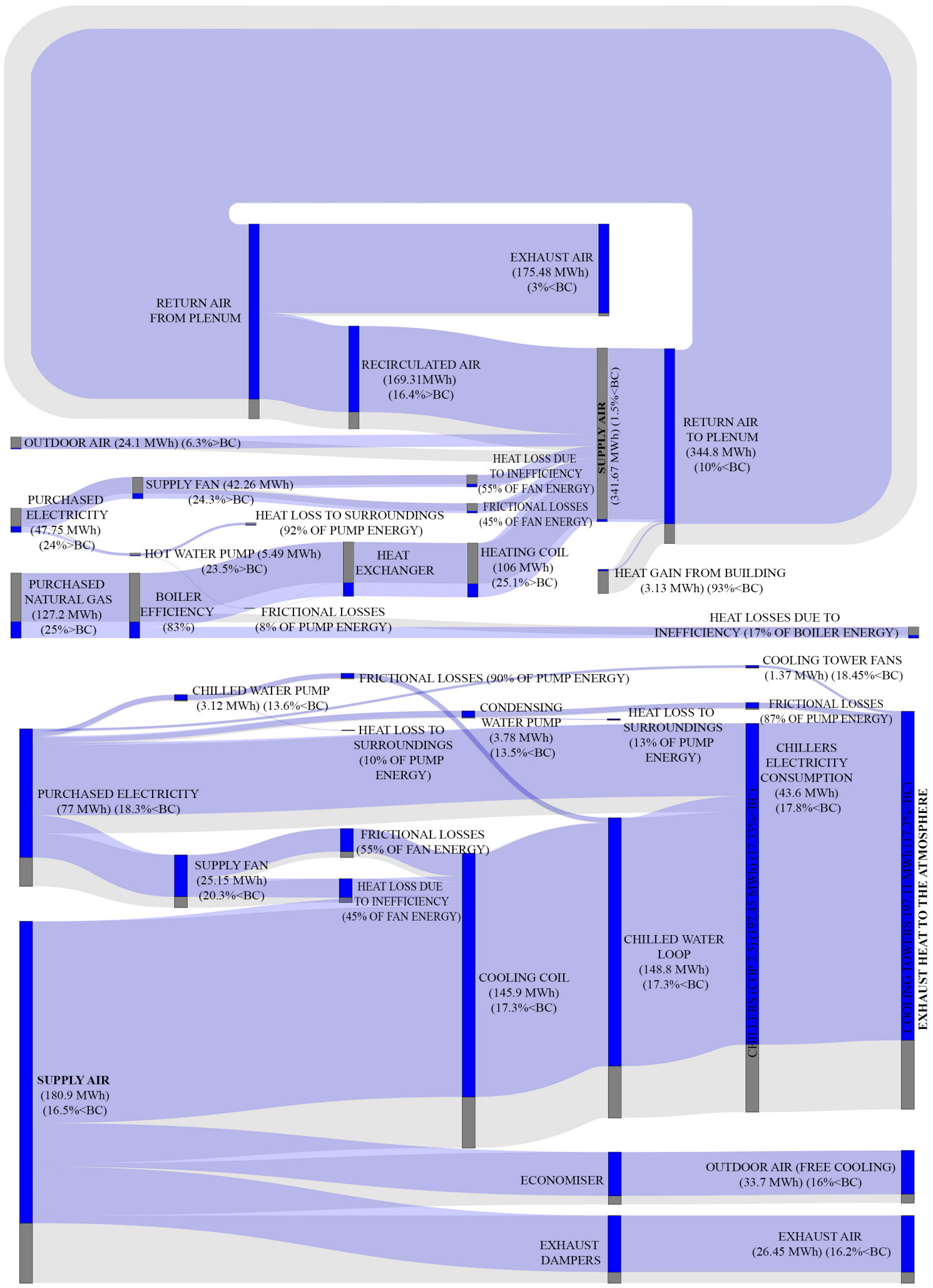

Figure 6.8: Sankey diagrams showing energy flows (grey for BC and blue for I2) on HVAC for the winter season (top) and summer season (bottom) 

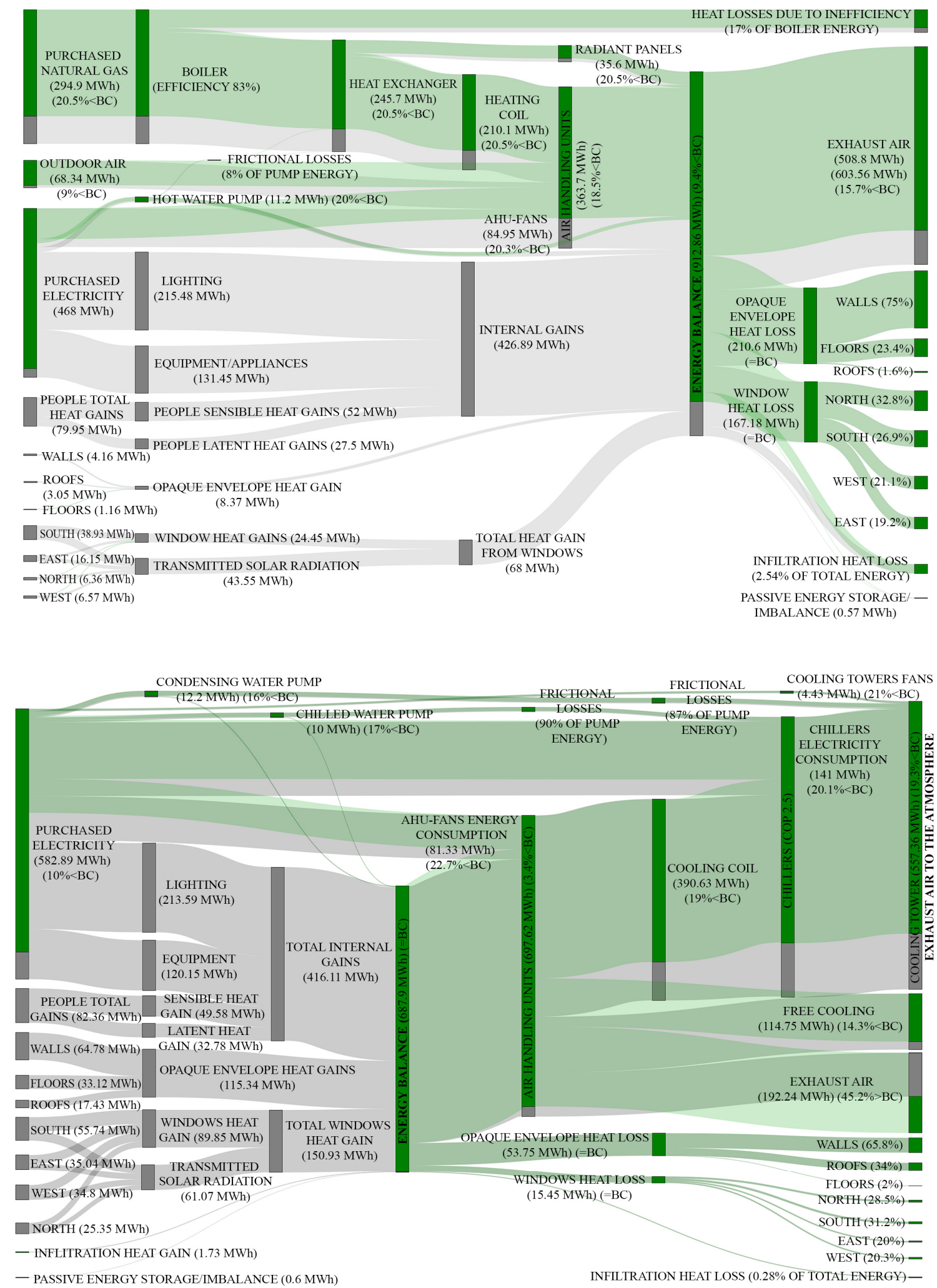

Figure 6.9: Sankey diagrams showing energy flows (grey for $\mathrm{BC}$ and green for I3) on building-level for the winter season (top) and summer season (bottom) 


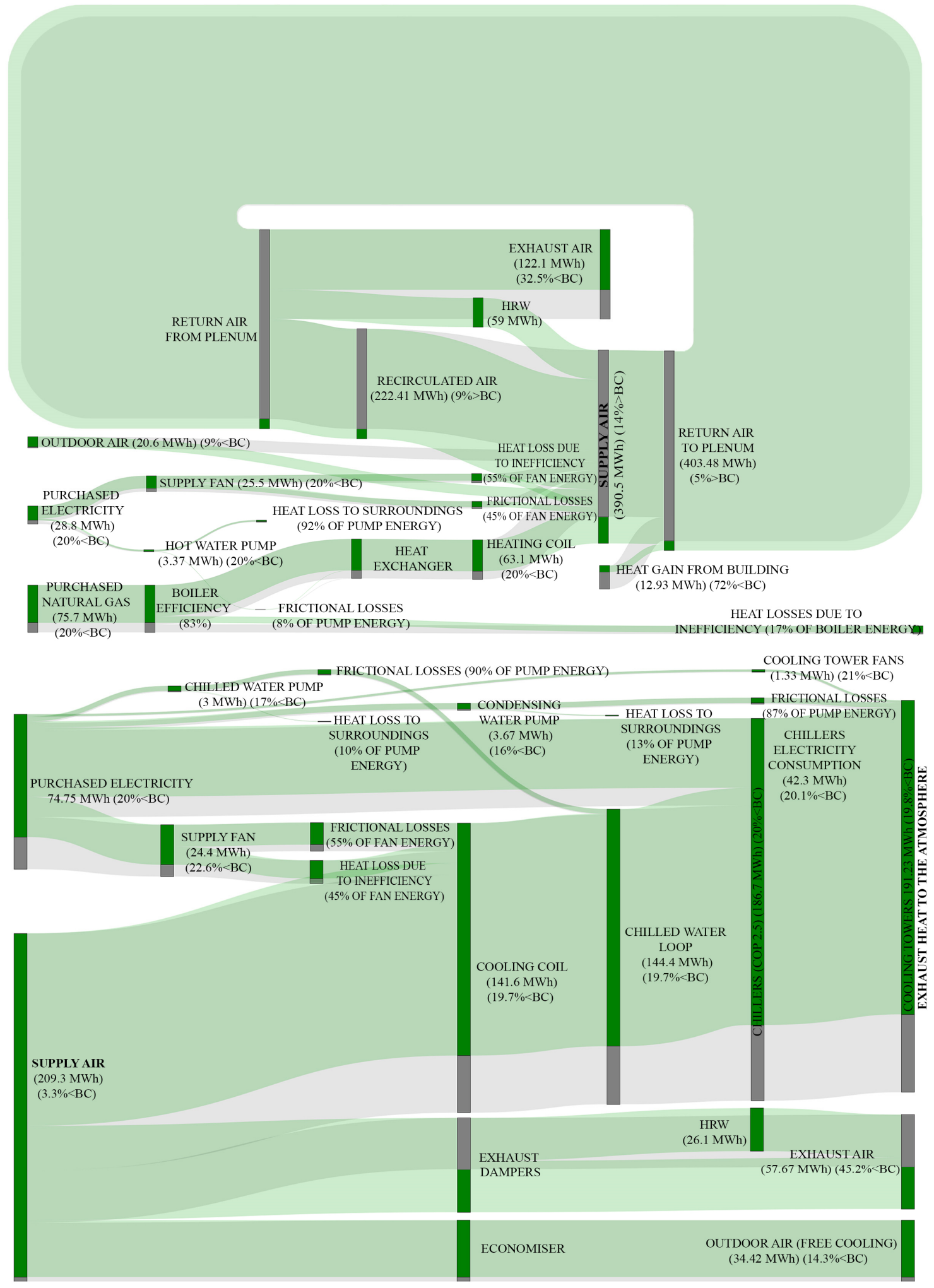

Figure 6.10: Sankey diagrams showing energy flows (grey for BC and green for I3) on HVAC for the winter season (top) and summer season (bottom) 
In the base case during summer season, the highest source $(\sim 31$ percent $)$ of heat gain was from lighting. Thus, the second design variant (I2) aimed at reducing lighting power density by 50 percent. As a result, during summer season, the electric energy consumption by AHU-fans, cooling tower fans, chillers, chilled water pump, and condensing water pump were reduced by $20.3,18.45,17.8,13.6$, and 13.5 percent compared to base case, respectively. However, during winter days, this reduction in internal gains from lighting caused an increase of 23 percent to natural gas consumption by the boiler. Despite the increase in heating energy, the lighting power consumption was reduced by 50 percent for both seasons and substantial reduction were recorded for cooling system components.

In the base case, the exhaust air from AHU was responsible for approximately 60 percent of heat loss during winter days due to that there is no HRW installed. Thus, the third design variant (I3) focused on installing heat recovery wheel (HRW) in the air handling units in order to take the advantage of the hot and cold air exhausted from the AHU in order to save energy required for heating and cooling. During winter days, the natural gas energy consumption was reduced by 20.5 percent compared to base case. While during summer days, the chillers electric energy consumption was reduced by 20.1 percent.

Compared to pie charts and other conventional graphs, Sankey diagrams facilitate the visualization of not only proportions but also the direction of flow of energy within buildings and their systems. For instance, Sankey diagrams were able to visualize the amount of lighting energy to the total internal heat gains. Moreover, it can visualize the amount of mechanical cooling required to remove internal heat gains. Moreover, Sankey diagrams demonstrated its usability and effectiveness to compare one design variant at a 
time or all design variants in one diagram. This could help different users evaluating different design alternatives.

\subsection{Summary and Future Work}

The aim of this study was to investigate the feasibility of utilizing Sankey diagrams in visualizing building energy performance obtained from simulation outputs. The study proposed a workflow to obtain, analyze, and visualize energy flows obtained from simulation outputs Large office reference building model that complies with the national energy code of Canada for buildings for Ottawa climate zone was selected as the base case model. Different design variants were proposed and tested against the base case model independently. The proposed Sankey diagrams helped in visualizing building energy performance and to understand the upstream and downstream impact to allow various design variants to be evaluated by users (such as architects, and design engineers). Moreover, it helped in visualizing the impact of changing building/system components on the whole system performance and demonstrate energy-saving strategies.

One of the challenges in creating Sankey diagrams was creating feedback loops. The D3's Sankey layout code used has some limitations in creating feedback loops, so these loops were added manually using image editing software.

Future steps for this research include: developing tool(s) for automating the process that creates Sankey diagrams from simulation output files, and developing interactive Sankey diagrams by allowing the user to select the spatial and temporal resolutions (discussed in Chapter 7). 


\section{Chapter 7: Automating the Creation of Sankey Diagrams}

\subsection{Introduction}

Once the workflows and methodologies for creating Sankey diagrams at various levels of the building environment, the process was automated for better dissemination and future application. This chapter provides the detailed methods that were developed to automate this process. Data obtained from energy simulation (EnergyPlus) outputs were used to create Sankey diagrams on different spatial (i.e. building-level and HVAC system level) and temporal (i.e. monthly, daily, and hourly) resolutions.

The first step in creating Sankey diagrams on different spatial and temporal resolutions is to select the appropriate output variables from energy simulation tools (EnergyPlus in this case). The required output variables and meters to create Sankey diagrams are discussed in Section 7.3. In order to test different building systems, large, medium, and small office reference building models that complies with the National Energy Code of Canada for Buildings (NECB) for Ottawa climate zone were selected.

The purpose of this framework is to automate the process of creating Sankey diagrams from energy simulation outputs. The framework entails the development of a user-graphical interface web page using Hypertext Preprocessor (PHP), JavaScript, and Hypertext Markup Language (HTML). The user at first inserts the IDF file (without output variables and meters) in the web page and selects the building type (from a drop-down menu) that corresponds to the same in the IDF file. The output variables and meters will be appended to the EnergyPlus IDF file using PHP code. The user should then run the generated IDF file in EnergyPlus V8.6 on local machine and import the simulation output 
files in the web page. EnergyPlus generates comma separated values (CSV) and ESO files that contains the simulation results. However, CSV files are limited to 255 columns (i.e. variables), thus ESO files were selected for the purpose of this study as it can contain all output variables and meters. In the future, a framework will be developed to execute EnergyPlus on a cloud to automate the process of obtaining ESO files. The PHP code then analyzes the ESO file by matching certain strings and patterns in the IDF file. The PHP code then generates Comma Separated Value (CSV) files that will be read by JavaScript code to generate Sankey diagrams. The user can control the displayed diagrams including number of diagrams, colors, and fonts. The user also can select spatial resolution from a drop-down menu (i.e. building-level and HVAC system) and temporal resolution (i.e. monthly, daily, and hourly) using a slide bar. Figure 7.1 shows the framework to automate the process of creating Sankey diagrams from EnergyPlus IDF and simulation output (ESO) files.

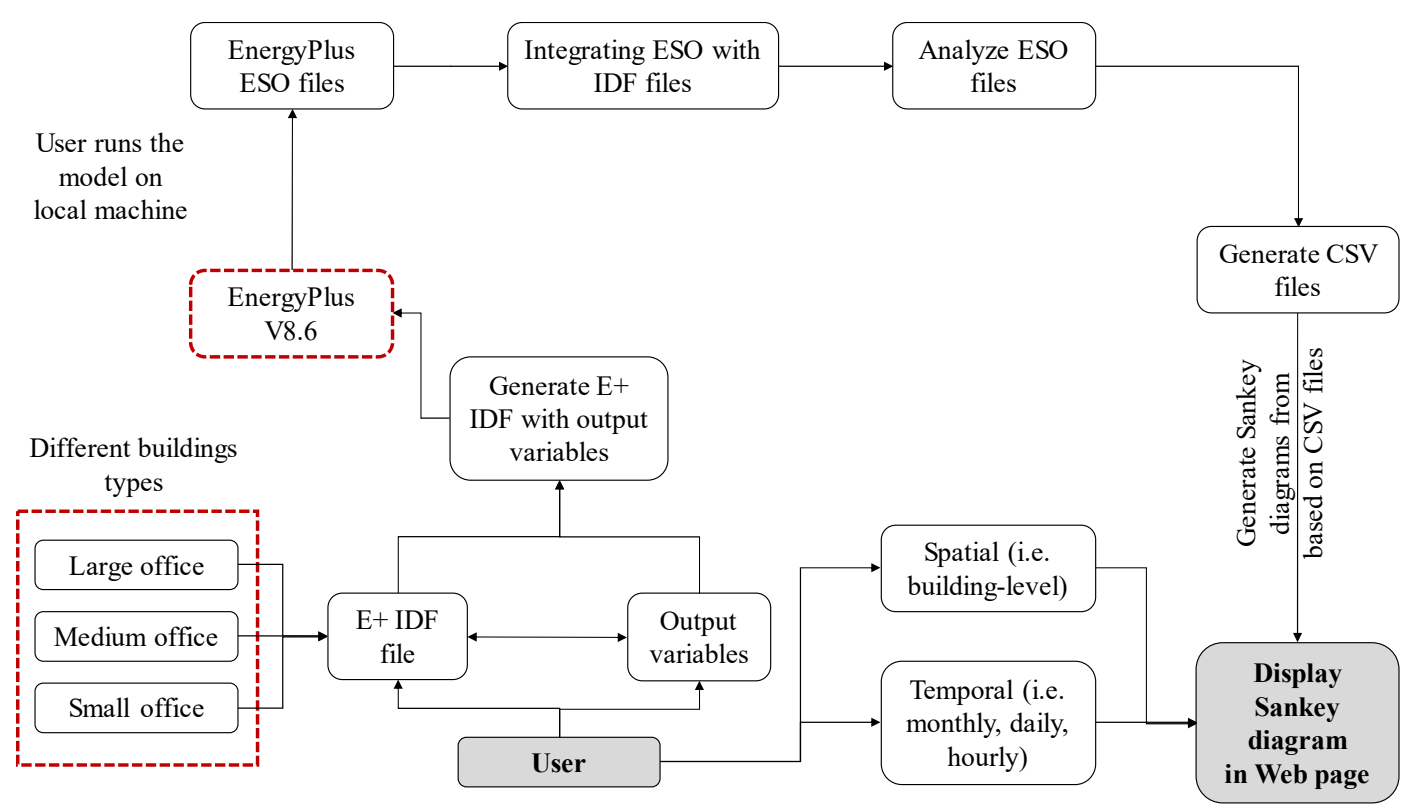

Figure 7.1: flowchart showing the framework to automate the process of creating Sankey diagrams from EnergyPlus IDF and simulation output (ESO) files 
The following sections illustrate: 1) EnergyPlus IDF files used in the study, 2) the required output variables to create Sankey diagrams, 3) front-end implementation including HTML and JavaScript codes, and 4) back-end implementation including PHP code.

\subsection{EnergyPlus IDF}

The purpose of this section is to provide information on the selected EnergyPlus V8.6 office building models included in this study. Large, medium, and small office reference buildings that complies with the National Energy Code of Canada for Buildings (NECB) for Ottawa climate zone [251] were selected for the purpose of testing and validation of the code developed on different building systems. Figure 7.2 shows large, medium, and small office building models used in the study. Table 7.1 shows large, medium, and small office building model details summary. AMY (actual meteorological year) weather data was used for Ottawa, Canada [202]. Schedules for internal gains used are based on American Society for Heating, Refrigerating and Air Conditioning Engineers (ASHRAE) Standard 90.1. 


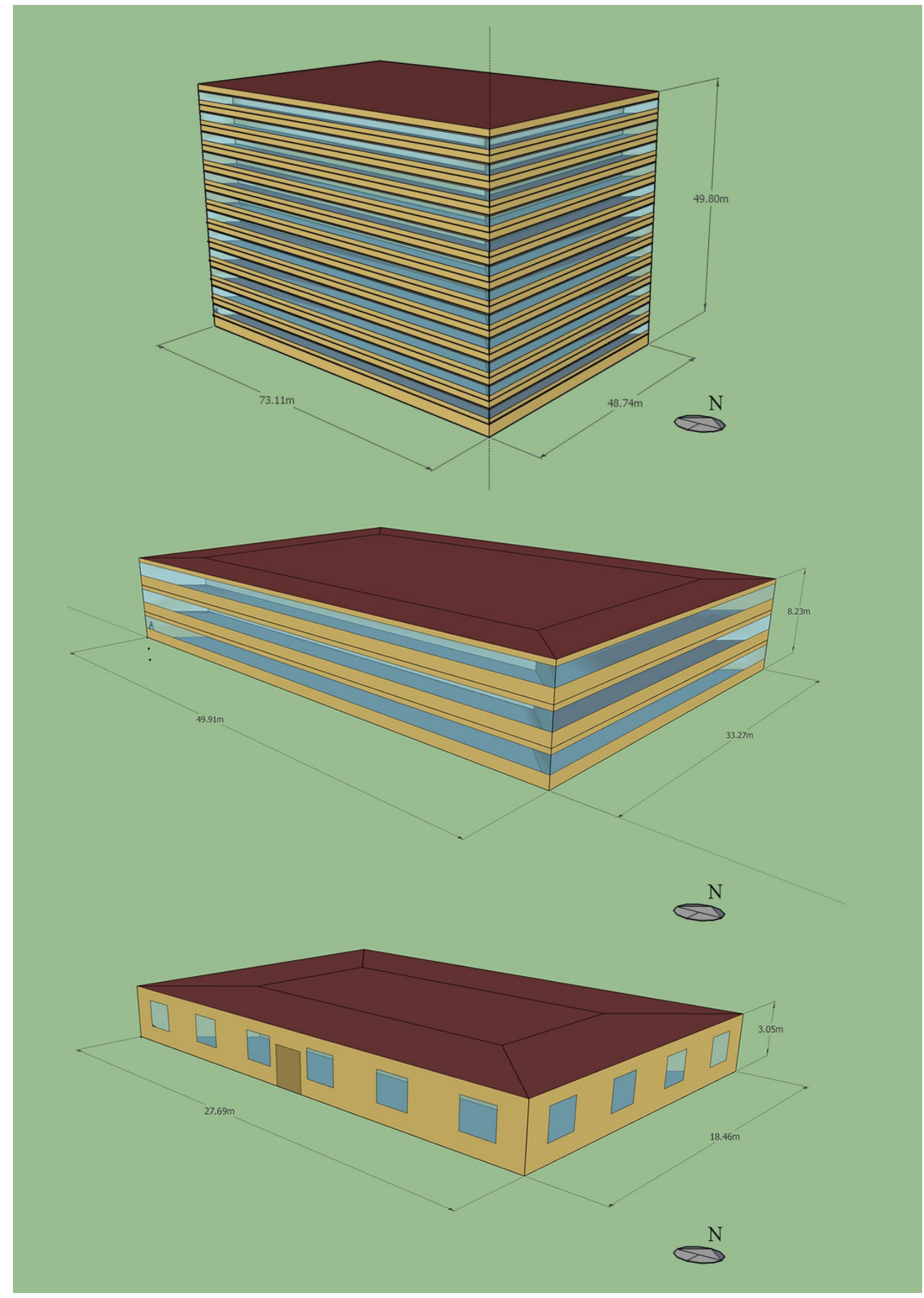

Figure 7.2: large (top), medium (middle), and small (bottom) office building models 
Table 7.1: Base case model details summary

\begin{tabular}{|c|c|c|c|c|}
\hline \multirow{5}{*}{ General } & Bldg. type & $\begin{array}{l}\text { Large office } \\
\text { building }\end{array}$ & $\begin{array}{c}\text { Medium } \\
\text { office } \\
\text { building }\end{array}$ & $\begin{array}{l}\text { Small office } \\
\text { building }\end{array}$ \\
\hline & Floor Area $\left(\mathrm{m}^{2}\right)$ & 14,252 & 4,982 & 511 \\
\hline & $\begin{array}{l}\text { No. of stories (including } \\
\text { basement) }\end{array}$ & 12 & $\begin{array}{l}3 \text { (no } \\
\text { basement) }\end{array}$ & $\begin{array}{l}1 \text { (no } \\
\text { basement) }\end{array}$ \\
\hline & $\begin{array}{l}\text { No. of thermal zones ( } 4 \\
\text { perimeters and } 1 \text { core } \\
\text { zones/typical floor) }\end{array}$ & 56 & 12 & 4 \\
\hline & $\begin{array}{l}\text { Window to wall ratio } \\
\text { (WWR) for all } \\
\text { orientations: }\end{array}$ & 44.57 & 47.68 & 19.81 \\
\hline \multirow{6}{*}{$\begin{array}{c}\text { Building } \\
\text { Envelope } U \text { - } \\
\text { value } \\
\left(\mathrm{W} / \mathrm{m}^{2} \cdot \mathrm{K}\right)\end{array}$} & Roof & \multicolumn{3}{|c|}{1.881} \\
\hline & Walls & \multicolumn{3}{|c|}{0.487} \\
\hline & Below grade walls & 3.663 & N/A & N/A \\
\hline & $\begin{array}{l}\text { Intermediate floor and } \\
\text { basement floor }\end{array}$ & \multicolumn{3}{|c|}{1.881} \\
\hline & $\begin{array}{l}\text { Windows (double glazed } \\
(6 \mathrm{~mm}) \text { with } 6 \mathrm{~mm} \text { air gap: } \\
\text { clear from inside and } \\
\text { tinted from outside) }\end{array}$ & \multicolumn{3}{|c|}{3.045} \\
\hline & Window (SHGC) & \multicolumn{3}{|c|}{0.368} \\
\hline \multirow{3}{*}{ Internal Gains } & $\begin{array}{l}\text { People: } \\
\text { office } / \text { mechanical rooms } \\
\left(\text { person } / \mathrm{m}^{2}\right)\end{array}$ & $0.05 / 0.00495$ & \multicolumn{2}{|c|}{$0.05 / \mathrm{N} / \mathrm{A}$} \\
\hline & $\begin{array}{l}\text { Lighting: } \\
\text { office/mechanical rooms } \\
\left(\mathrm{W} / \mathrm{m}^{2}\right)\end{array}$ & $10.9 / 13.29$ & $10.9 / \mathrm{N} / \mathrm{A}$ & $11.9 / \mathrm{N} / \mathrm{A}$ \\
\hline & $\begin{array}{l}\text { Equipment: } \\
\text { office/mechanical rooms } \\
\left(\mathrm{W} / \mathrm{m}^{2}\right)\end{array}$ & $7.5 / 1$ & \multicolumn{2}{|c|}{$7.5 / \mathrm{N} / \mathrm{A}$} \\
\hline $\begin{array}{l}\text { Infiltration } \\
\text { Rates }\end{array}$ & $\begin{array}{l}\text { Floor per exterior surface } \\
\text { area }\left(\mathrm{m}^{3} / \mathrm{s}-\mathrm{m}^{2}\right)\end{array}$ & \multicolumn{3}{|c|}{0.00025} \\
\hline $\begin{array}{l}\text { HVAC air } \\
\quad \text { loop }\end{array}$ & \multicolumn{2}{|c|}{$\begin{array}{c}\text { Four air handling units (AHUs). Air } \\
\text { distribution system is single duct VAV-box } \\
\text { with reheat coils }\end{array}$} & $\begin{array}{c}\text { Three air } \\
\text { handling } \\
\text { units } \\
\text { (AHUs). Air } \\
\text { distribution } \\
\text { system is } \\
\text { single duct } \\
\text { VAV-box } \\
\text { with reheat }\end{array}$ & $\begin{array}{l}\text { The building is } \\
\text { equipped with } \\
\text { five Packaged } \\
\text { Single-Zone }\end{array}$ \\
\hline
\end{tabular}




\begin{tabular}{|c|c|c|}
\hline Space Cooling & $\begin{array}{c}\text { Two electric EIR chillers with COP of } 2.5 \text { and a cooling } \\
\text { tower single speed. The building relies on free outdoor } \\
\text { cooling when the outdoor temperature is below } 28^{\circ} \mathrm{C}\end{array}$ & $\begin{array}{c}\text { Cooling DX } \\
\text { single unit }\end{array}$ \\
\hline $\begin{array}{c}\text { Space } \\
\text { Heating/Hot } \\
\text { water }\end{array}$ & $\begin{array}{c}\text { Hot water delivered from a boiler using natural gas (83\% thermal efficiency). } \\
\text { All zones are equipped with radiant panels }\end{array}$ \\
\hline $\begin{array}{c}\text { Thermostat } \\
\text { settings }\end{array}$ & $\begin{array}{c}\text { heating/cooling setpoints are } 22^{\circ} \mathrm{C} \text { to } 24^{\circ} \mathrm{C} \text {, respectively. } \\
\text { heating/cooling setbacks are } 18^{\circ} \mathrm{C} \text { to } 27^{\circ} \mathrm{C} \text {, respectively. }\end{array}$ \\
\hline
\end{tabular}

\subsection{Simulation Output Variables and Meters}

This section aims at providing simulation output variables and meters that will be used to create Sankey diagrams for large, medium, and small office reference buildings. The objective of the PHP code (discussed in Section 7.5.1) is to append the output variables and meters with the IDF file imported by the user. Table 7.2 shows hourly simulation output variables and meters required to create Sankey diagrams for the selected energy models.

Table 7.2: Hourly simulation output variables and meters used in the study

\begin{tabular}{|c|c|c|c|}
\hline Output variables & $\begin{array}{l}\text { Large office } \\
\text { building }\end{array}$ & $\begin{array}{l}\text { Medium office } \\
\text { building }\end{array}$ & $\begin{array}{c}\text { Small office } \\
\text { building }\end{array}$ \\
\hline Cooling tower & \multicolumn{2}{|c|}{$\begin{array}{l}\text { Cooling Tower Fan Electric Energy } \\
\text { Cooling Tower Heat Transfer Rate }\end{array}$} & $\mathrm{N} / \mathrm{A}$ \\
\hline Pump & \multicolumn{2}{|c|}{$\begin{array}{l}\text { Pump Electric Energy } \\
\text { Pump Fluid Heat Gain Energy }\end{array}$} & $\begin{array}{l}\text { Note: No condensing } \\
\text { or chilled pump are } \\
\text { installed. Only hot } \\
\text { water pump is } \\
\text { included }\end{array}$ \\
\hline AHU & \multicolumn{3}{|c|}{$\begin{array}{l}\text { System Node Mass Flow Rate } \\
\text { System Node Enthalpy } \\
\text { Air System Humidifier Electric Energy } \\
\text { Air System Humidifier Gas Energy }\end{array}$} \\
\hline Heating Coils/VAV- & \multicolumn{2}{|c|}{ Heating Coil Heating Energy } & $\mathrm{N} / \mathrm{A}$ \\
\hline
\end{tabular}




\begin{tabular}{|c|c|c|c|}
\hline reheat coils & & & \\
\hline People & \multicolumn{3}{|c|}{$\begin{array}{l}\text { People Sensible Heating Energy } \\
\text { People Latent Gain Energy }\end{array}$} \\
\hline Windows & \multicolumn{3}{|c|}{$\begin{array}{l}\text { Zone Windows Total Transmitted Solar Radiation Rate } \\
\text { Zone Windows Total Heat Gain Rate } \\
\text { Zone Windows Total Heat Loss Rate }\end{array}$} \\
\hline Infiltration & \multicolumn{3}{|c|}{$\begin{array}{l}\text { Zone Infiltration Total Heat Gain Energy } \\
\text { Zone Infiltration Total Heat Loss Energy }\end{array}$} \\
\hline Opaque envelope & \multicolumn{3}{|c|}{$\begin{array}{l}\text { Surface Inside Face Conduction Heat Gain Rate } \\
\text { Surface Inside Face Conduction Heat Loss Rate } \\
\text { Surface Heat Storage Gain Rate } \\
\text { Surface Heat Storage Loss Rate }\end{array}$} \\
\hline Output meters & $\begin{array}{l}\text { Large office } \\
\text { building }\end{array}$ & $\begin{array}{c}\text { Medium office } \\
\text { building }\end{array}$ & $\begin{array}{l}\text { Small office } \\
\text { building }\end{array}$ \\
\hline Boiler & \multicolumn{3}{|c|}{$\begin{array}{l}\text { Boiler:Heating:Gas } \\
\text { Boilers:EnergyTransfer } \\
\text { Boiler Parasitic:Heating:Electricity }\end{array}$} \\
\hline $\begin{array}{l}\text { Chillers / DX unit (for } \\
\text { small office) }\end{array}$ & \multicolumn{3}{|c|}{ Cooling:Electricity } \\
\hline Cooling Coils & \multicolumn{3}{|c|}{ CoolingCoils:EnergyTransfer } \\
\hline Heating Coils & \multicolumn{2}{|c|}{$\mathrm{N} / \mathrm{A}$} & $\begin{array}{l}\text { HeatingCoils:Energy } \\
\text { Transfer }\end{array}$ \\
\hline AHU-fans & \multicolumn{3}{|l|}{ Fans:Electricity } \\
\hline Baseboard & \multicolumn{3}{|c|}{ Baseboard:EnergyTransfer } \\
\hline Lighting & \multicolumn{3}{|c|}{ InteriorLights:Electricity } \\
\hline Equipment & \multicolumn{3}{|c|}{ InteriorEquipment:Electricity } \\
\hline
\end{tabular}

Some of the variables and meters mentioned in the above table could be read directly in order to create elements in Sankey diagrams. However, some other variables need mathematical computation in order to be used to create Sankey diagrams. For instance, the "Pump Electric Energy" variable represents the electricity consumption for the chilled, condensing, and hot water loop pumps. As the name of the pumps varies from 
one IDF file to another and also are not explicitly mentioned in the ESO file. Thus, a processing step was conducted using PHP code to search for the name of the pump in the IDF file and determine the corresponding name of the pump in the ESO file. The same procedure has been conducted for several other variables (i.e. heating coils, AHU-System Node Mass Flow Rate and Node Enthalpy, Windows, and Opaque Envelope). Section 7.5.1 addresses the mathematical computations applied to these variables using PHP code.

\subsection{Front-end Implementation}

This section explains the front-end implementation of the user-graphical interface web page developed including Hypertext Markup Language (HTML) and JavaScript codes.

\subsubsection{Hypertext Markup Language (HTML)}

HTML is the set of markup symbols or codes inserted in a file intended for display on a World Wide Web browser page. The purpose of this code is to organize the structure of the web page including controls (such as slide bar, number of diagrams, upload files, colors, and fonts). HTML includes JavaScript library (jQuery) for advanced processing. The HTML code includes Cascading Style Sheets (CSS) for styling the web page elements. The user will be using the web page to add Sankey diagrams including names, upload the IDF files, and select the building type (i.e. large, medium, and small). In order to generate IDF file with the required outputs, a PHP code (discussed in Section 7.5.1) was used to append the required variables and meters (mentioned in Section 7.3) into the IDF files. The user then runs the generated IDF file in EnergyPlus V8.6 and imports the ESO file in the web page. The uploaded ESO file will be analyzed along with the IDF file using PHP code (discussed in Section 7.5.1) in order to create CSV files required to construct Sankey 
diagrams. D3 (Data-Driven Documents) JavaScript library was used to read CSV files generated from PHP code to create Sankey diagrams (discussed in Section 7.4.2). The user then can select spatial and temporal resolutions from the web page. Appendix D shows the HTML code developed the in this study.

\subsubsection{JavaScript}

JavaScript resides inside Hypertext Markup Language (HTML) documents, and can provide levels of interactivity to web pages that are not achievable with simple HTML. Common uses for JavaScript are image manipulation, form validation, and dynamic changes of content. The purpose of the JavaScript is to generate nodes and links between elements. D3 (Data-Driven Documents) JavaScript library was used for that purpose. D3 is a JavaScript library for visualizing data with Hypertext Markup Language (HTML), Scalable Vector Graphics (SVG), and Cascading Style Sheets (CSS). The script developed in this part relied on D3's Sankey layout code developed by Google Developers, but was further developed to fulfil the objectives of the study [183]. The JavaScript code reads Comma Separated Values (CSV) generated from the ESO files in order to create Sankey diagrams. The code enables the user to modify node width, height, link curvature, and to adjust transformation of nodes and links. In addition, the code has the ability to create multiple diagrams laying over each other.

JavaScript was also used to create functions for the elements created in the HTML code (such as generate and download IDF files, upload ESO file, add and remove diagrams, selecting building type, and generate CSV files). Moreover, JavaScript was used to provide the controls for the elements created in the HTML code (such as load data from CSV files, construct Sankey diagram(s), slide bar functions (navigate through different hours, days, 
and months), adding labels to slider, select spatial resolution, changing color of diagrams, and transform nodes and links of Sankey diagrams).

The following list of appendices shows JavaScript codes developed in the study:

\begin{tabular}{|c|l|}
\hline Appendix & \multicolumn{1}{|c|}{ Description of the JavaScript codes } \\
\hline E1 & $\begin{array}{l}\text { Shows functions and controls for the "HOME" tab including: 1) } \\
\text { select number and name of diagrams, 2) upload IDF file, 3) select } \\
\text { building type from a drop-down menu, 4) generate IDF file with the } \\
\text { required outputs and meters, 5) upload ESO file, 6) generate CSV } \\
\text { files, and 7) remove diagrams. }\end{array}$ \\
\hline E2 & Shows D3's Sankey layout JavaScript code \\
\hline E3 & Shows list of variables that are used by other JavaScript codes \\
\hline E4 & $\begin{array}{l}\text { Generate multiple Sankey diagrams based on the number of } \\
\text { diagrams selected by the user (in the HOME tab) }\end{array}$ \\
\hline E5 & Shows functions and controls to select spatial resolution \\
\hline E6 & $\begin{array}{l}\text { Shows functions and controls to select temporal resolution using } \\
\text { slide bar }\end{array}$ \\
\hline E7 & Shows functions and controls to animate the results \\
\hline E8 & Select color for each diagram \\
\hline E9 & Show/hide diagrams from a drop-down menu \\
\hline E10 & Shows functions to initialize HTML elements in the web page \\
\hline
\end{tabular}

\subsection{Back-end Implementation}

This section explains the back-end implementation of the user-graphical interface web page developed including Hypertext Preprocessor (PHP) code.

\subsubsection{Hypertext Preprocessor (PHP)}

PHP is a popular general-purpose scripting language that is especially suited to web development. The purpose of the PHP code is to perform operations on the files uploaded in the web page by the user (i.e. IDF and ESO files). The operations performed are: 1) generate IDF files with the required output variables, 2) analyze vast data sets resulting from EnergyPlus simulation outputs (ESO files), 3) generate CSV files, and 4) check and correct the energy balance. Appendix F shows the PHP code developed in this study.

\section{1) Generate IDF files with the required output variables and meters}


The purpose of this operation is to append the output variables and meters that corresponds to the building type selected (i.e. large, medium, and small office) mentioned in Section 7.3 into the IDF file imported by the user. This will ensure that the required outputs to create Sankey diagrams are included in the IDF file.

\section{2) Analyze ESO files}

The purpose of this operation is to analyze some of the output variables in the ESO file in order to be used in the CSV files that will be read by JavaScript code to create Sankey diagrams. The following discussion includes the output variables that needs mathematical computations in order to be used to create Sankey diagrams.

For the pumps, the name of the pumps may vary from one file to another and may not reflect the type of the pump (i.e. chilled, condensing, and hot water). Thus, in order to determine the name of the pumps (in the ESO file) that corresponds to the pump type, the code navigates to "ALL OBJECTS IN CLASS: BRANCH" section in the IDF file and searches for the pump type (pump: constant speed in this case) and then searches in the following branch elements for plant equipment (i.e. chillers, cooling towers, and boilers). The code then determines the name of the pump in the IDF file and extracts the data from the ESO file.

In the case of large and medium office buildings, the zones are heated by VAVreheat coils and heating coils in the air handling units (AHUs). In order to determine the energy consumption by AHU-heating coils and VAV-reheat coils, the code searches for the name of the AHU-heating coils in the IDF file. This could be done by navigating to "ALL OBJECTS IN CLASS: BRANCH" section in the IDF file and searches for “AirLoopHVAC:OutdoorAirSystem" section under "object type" and then searches for the 
name of the heating coils based on the number of AHUs in the IDF file. The code then extracts the AHU-heating coils energy consumption from the ESO file. The code then sums up all the remaining heating coils in the ESO file, which represents the VAV-reheat coil energy consumption. On the other hand, for the small office the packaged single-zoneheating coils energy consumption data are directly extracted from output meter as mentioned in Table 7.2.

EnergyPlus provides mass flow rate and enthalpy for system nodes. The name of nodes in the IDF file may vary from one file to another. Thus, in order to determine the energy flows for outdoor, mixed, return, and exhaust air in the AHU, the code at first searches for the name of the air stream nodes that corresponds to the exhaust (relief), outdoor, mixed, and return air under "ALL OBJECTS IN CLASS: OUTDOORAIR: MIXER" section. The code then extracts the product of mass flow and enthalpy of each node.

In order to determine windows total transmitted solar radiation rate, total heat gain rate, and total heat loss rate for each cardinal direction. Information such as cardinal directions are not included in the IDF file, thus the user should add the cardinal directions (i.e. East, West, South, and North) to the name of the fenestration surfaces. The code searches for the outputs in the ESO file (i.e. zone windows total transmitted solar radiation rate, zone windows total heat gain rate, and zone windows total heat loss rate) and searches for the cardinal directions for each element. The code then sums up the output for each cardinal direction.

For the conduction heat gain and loss rates, the output variables include all surfaces 
including exterior and interior opaque envelope components. However, the purpose is to obtain the amount of heat gain and loss by conduction through exterior opaque envelope components. Thus, the code navigates to "BUILDING_SURFACE_DETAILED_ SECTION" section in the IDF file. The code then searches for "Outside Boundary Condition Object" that is null. The code then searches for the "Surface Type" and determine the name of the opaque surface that corresponds to the building envelope component (wall, roof, and floor) to be extracted from the ESO file.

For other variables, such as (people sensible heating energy, people latent gain energy, zone infiltration total heat gain energy, zone infiltration total heat loss energy, air system humidifier gas energy, surface heat storage loss rate, and surface heat storage gain rate) provides output for each zone or element. Thus, the code was developed to sum up zones or elements for each output.

\section{3) Generate CSV files}

In order to create Sankey diagrams, CSV files should be created including (source, target, and values) in separate columns. The purpose of this code is to create CSV files that contains hourly, daily, and monthly data. However, CSV files can only contain 255 columns, thus data for each week (i.e. 168 columns, which is the number of hours per week) was created in separate files (i.e. 53 files). The last CSV (i.e. 53) includes 24 columns (hours) to include the last day of the year. Moreover, two CSV files were created to include the daily data (i.e. 365 days) and one CSV file containing monthly data (i.e. 12 months).

\section{4) Check and correct the energy balance}

EnergyPlus provides dynamic simulation results (i.e. not steady-state). For 
instance, the simulation output variables provide the amount of energy in, energy out, stored energy, and released stored energy each time step. The amount of stored energy depends on the specific heat capacity of materials, equipment, and fluids. Thus, the aim of this process is to check and correct the energy balance across each component in the Sankey diagrams (e.g. energy consumption by radiant panels and heating coils should be equal to the amount of boiler energy transfer). Moreover, this process ensures that the energy balance is maintained on the building-level (i.e. the amount of energy entering and leaving the building is equal). Thus, output variables such as energy consumption by radiant panel and heating coils, energy extraction by cooling coils, and passive energy storage were modified to maintain energy balance for each hour and also maintain daily and monthly energy consumption.

\subsection{Discussion}

The main aim of the developed framework was to automate the process of creating Sankey diagrams from EnergyPlus outputs. The framework involves the development of a user-graphical interface web page using PHP, HTML, and JavaScript codes. The web page consists of two tabs: "HOME" and "Sankey Diagrams". In the "HOME" tab, the user can add the diagram name, number of diagrams, upload IDF file, select building type, and generate IDF file with the required output variables and meters used to create Sankey diagrams. Figure 7.3 shows the "HOME" tab of the web page. When the user selects "Generate IDF File", the generated IDF file with the required output variables and meters will be downloaded automatically. The user then imports the generated IDF file in EnergyPlus V8.6 on local machine to obtain the simulation results (ESO files). There is an additional feature where the user can remove the created diagrams to clear the created 
temporary folders and files.

\section{Implementation of Representation of Reference}

Building Energy Model Performance Using Sankey Diagrams

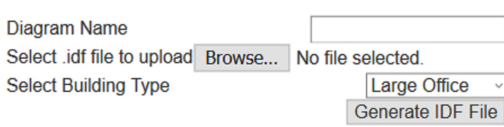

List of Sankey Diagrams

Select .idf file to upload Browse... No file selected.

Generate IDF File

Remove All Diagrams

Figure 7.3: "HOME" tab showing different user-graphical interface features

The next step is to upload the ESO file in the desired location (in case of having multiple diagrams) as shown in Figure 7.4.

Home

Sankey Diagrams

Implementation of Representation of Reference

Building Energy Model Performance Using Sankey Diagrams

Diagram Name

Case 1

Select idf file to upload Choose File Ottawa Large ... building.idf

Select Building Type

Large Office

Diagram Name:Base Case

Building Type:Large Office

Upload ESO file

Choose File No file chosen

Remove Diagram

Diagram Name:Case 1

Building Type:Large Office

Upload ESO file

Choose File No file chosen

Remove Diagram

List of Sankey Diagrams

en

(1)

Figure 7.4: uploading of ESO file for each diagram

After uploading the ESO files, the user should click on "Generate CSV" to create CSV files that will be read by JavaScript to generate Sankey diagrams. In order to display the created diagrams, the user should select the "Sankey Diagrams" tab. In this tab, the Sankey diagrams are displayed. The user can choose the spatial resolution (i.e. buildinglevel and HVAC system) from the drop-down menu. The user can select the temporal 
resolution (i.e. monthly, daily, and hourly) from the radio button beside the slide bars and navigates through different hours, days, and months. The user can also click on the "play/pause" button to animate the results. Colors of nodes and links of each diagram could also be modified by the user. The user can also organize the node locations. The user can hide/unhide the created Sankey diagrams from the drop-down menu to evaluate different design iterations simultaneously. Figure 7.5 shows the "Sankey Diagrams" tab of the web page. For the purpose of testing the functionality of the developed webpage, samples of Sankey diagrams for large office reference building model are presented. Moreover, two design iterations were selected to test multi-layer Sankey diagrams. The first design iteration (base case for large office building model: mentioned in Table 7.1) and the second iteration (Case A: reduce USI-value from 3.045 to $1.903 \mathrm{~W} / \mathrm{m}^{2} \mathrm{~K}$ and SHGC from 0.368 to 0.252). Figure 7.6 shows multi-layer Sankey diagrams to evaluate various design iterations simultaneously. 


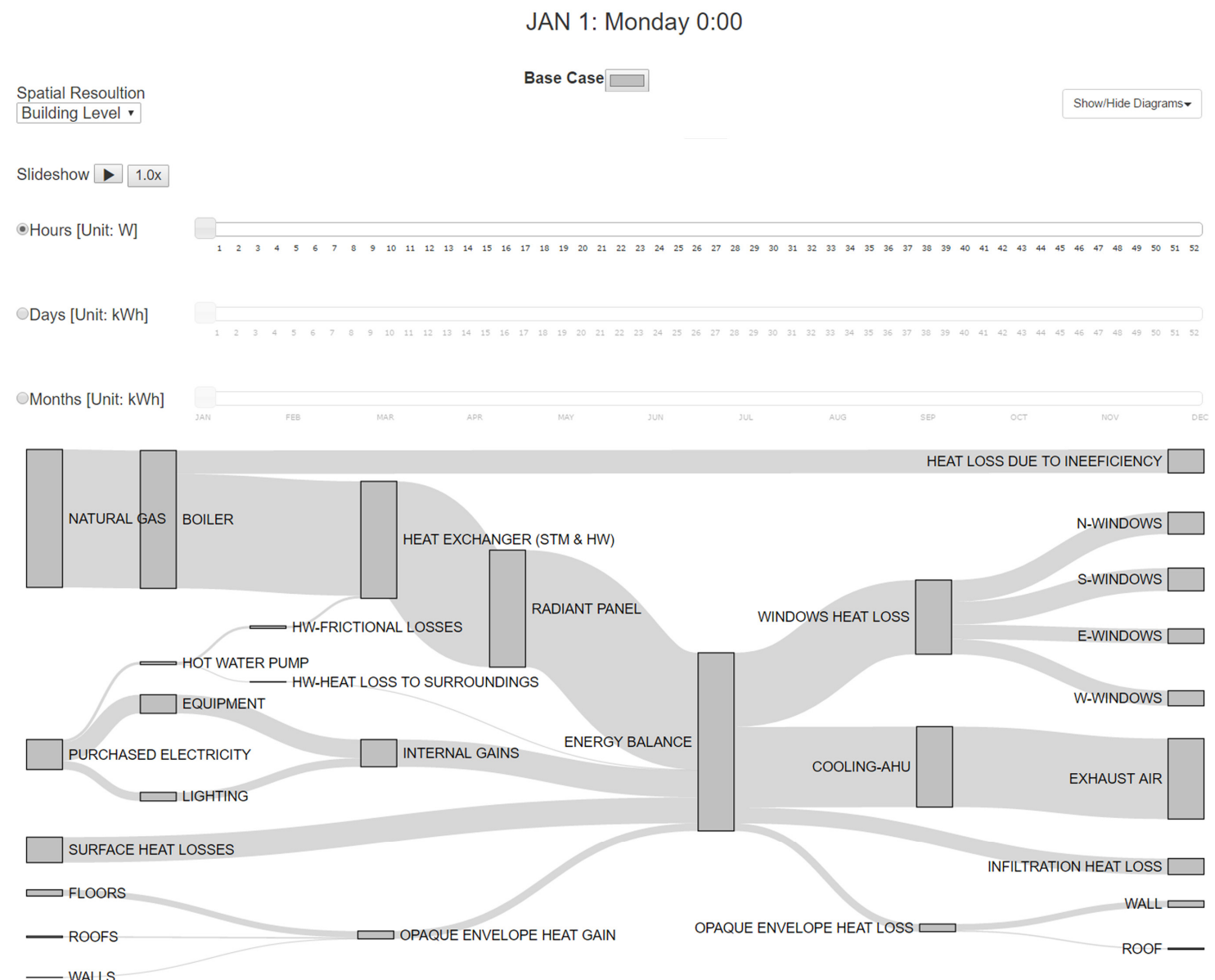

Figure 7.5: "Sankey Diagrams" tab showing different user-graphical interface features 


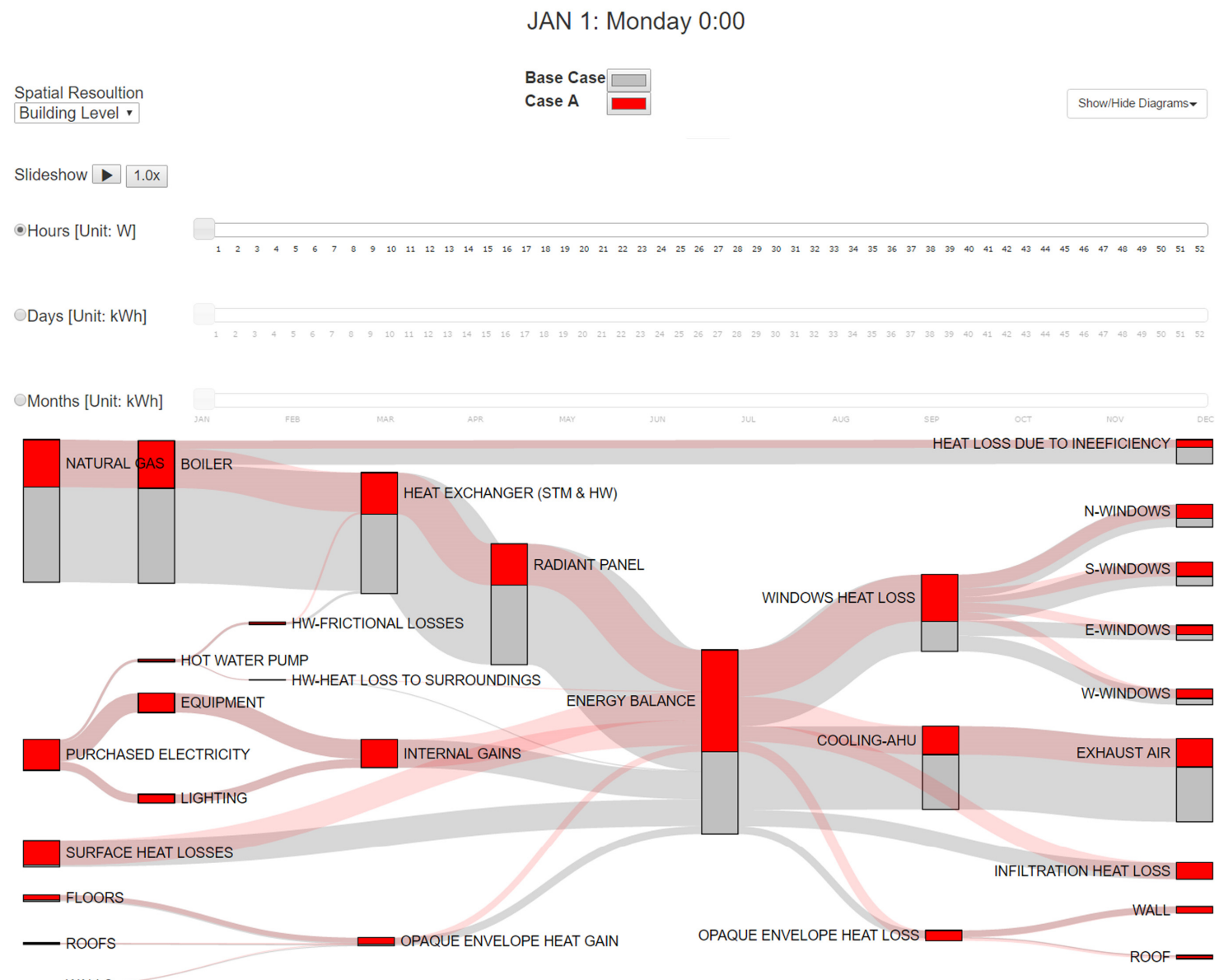

Figure 7.6: multi-layer Sankey diagrams for large office (including base case and case A)

\subsection{Summary and Future Work}

This chapter proposed a framework to automate the process of creating Sankey diagrams from energy simulation outputs through a user-graphical interface web page using Hypertext Preprocessor (PHP), JavaScript, and Hypertext Markup Language (HTML) codes. EnergyPlus v8.6 was selected as the energy simulation tool. Front-end (including HTML and JavaScript codes) and back-end (including PHP code) implementation of the web page were discussed. 
Different office reference building models (i.e. large, medium, and small) that complies with the National Energy Code of Canada for Buildings (NECB) for Ottawa climate zone were selected to test different building systems. Details on the selected EnergyPlus IDF of large, medium, and small office reference building models were provided. Moreover, the required output variables and meters for each office type were provided.

The developed PHP code helped in 1) generating EnergyPlus IDF files with the required variables and meters used to create Sankey diagrams on the building-level and HVAC system, 2) analyzing energy simulation output (ESO) file by matching certain strings and patterns in the IDF file, and 3) generating CSV files that will be read by JavaScript code to construct Sankey diagrams. The purpose of the developed JavaScript was to read CSV files generated by the PHP code to generate Sankey diagrams. D3 JavaScript library was used to create nodes and links between elements. The HTML code developed helped in organizing the structure of the web page including controls (such as slide bar, number of diagrams, upload files, colors, and fonts).

Samples of Sankey diagrams showing energy flows on the building-level and HVAC system for large office building model were provided to show and test different features of the webpage. Key features include: 1) user graphical interface including selecting diagram name and number of diagrams, uploading of IDF and ESO files, select building type, generating IDF and CSV files, transformation of nodes, and color selection, 2) creating interactive Sankey diagrams (the user can select spatial and temporal resolutions from drop-down menu and slide bars, respectively), and 3) creating multiple Sankey diagrams to evaluate different design iterations simultaneously. 
Future steps for this research include: 1) developing a framework to execute EnergyPlus on a cloud to automate the process of obtaining ESO files, 2) developing a code to generate feedback loops (i.e. recovered energy) for HVAC system, 3) test the developed workflow on other building systems, 4) integrating the developed framework to automate the process of creating Sankey diagrams into commercial tools (such as Revit, OpenStudio, etc.), and 5) integrating Sankey diagrams to parametric building modelling tools (such as Grasshopper). 


\section{Chapter 8: Conclusion and Future Work}

University campuses include a large number of facilities and building functions, which acts as small communities. Frequently, their buildings' resource consumption is metered to various levels of spatial and temporal resolutions to attempt to track and reduce GHG emissions. As a result, many data sources are available on the building-level but less information could be obtained from these sensors and sub-meters. Moreover, the Facilities Management and Planning (FMP) department at Carleton University utilizes building energy dashboard tools to visualize historical and instantaneous data. However, accessing data logging systems are often inconvenient and difficult due to use of multiple systems and technologies of varying vintages. Moreover, some data cannot be obtained from meters due to high cost (such as solar gains, infiltration and ventilation rates, etc.). Furthermore, on the visualization side, the current data availability and visualization tools do not lend themselves to identification of inefficiencies and possible solutions. The current visualization tools are limited in providing greater understanding on how each building or system component affects the overall system performance.

This work is divided into two parts. The objective of the first part "Developing Methods to Enhance Campus Operations" was to develop a useful workflow to facilitate the decision-making by building operators, campus planners, and other stakeholders that could be generalized to other Canadian campuses communities and could be applied to other building functions and vintages. This work focused on developing methods to analyze and visualize energy and mass flows and the corresponding energy costs on different spatial (i.e. campus, building-level, and HVAC system level) and temporal (i.e. annual, monthly, and hourly) resolutions. The research then demonstrated the applicability of utilizing 
Sankey diagrams to identify opportunities for energy savings on different scales from campus to building system level.

On the campus scale, the aim of the developed methods was to facilitate a greater understanding of how each building performs and to identify underperforming buildings so that building operators and other stakeholders can investigate further. Historical data obtained from meters were used to analyze energy flows from primary to secondary (onsite) energy source. The research also demonstrated the upstream environmental and economic impacts of buildings' and campus performance.

On the building-level, the aim of the research was to provide a comprehensive energy-use assessment that could facilitate the decision making by building owners, operators, and other stakeholders. The aim of this work was to develop an integrated framework using measured and modeled data to estimate energy flows and the corresponding energy costs on the building-level. The framework consisted of 1) develop BIM model, 2) a method to convert BIM to BPS, and 3) a method to calibrate BPS models.

On the HVAC system level, the aim of the research was to make use of real-time and historical data obtained from BMS to facilitate the decision making by building operators to manage the operation of building system level. This was achieved by converting sparse sensor data into estimated energy and mass flows and the corresponding energy cost for major components of AHU and plant loops.

Sankey diagrams were utilized in the current work to visualize the data for energy, mass and the corresponding costs obtained from the above-mentioned methods on various scales (from campus to building system level). 
The developed methods could yield greater insights about opportunities for energy savings and inspect operational problems on various scales. Key components include: 1) determining renewable to non-renewable primary energy source, 2) determining the performance of central plant and different buildings (varying in age and function), 3) quantifying measured and unmeasured energy flows by different components on buildinglevel and building system level (i.e. HVAC), 4) revealing the impact of a single component to the whole system performance, 5) revealing system inefficiencies and generation, transmission, and distribution losses, 6) estimating GHG emissions, 7) quantifying feedback loops, and 8) determining energy use trends over time (i.e. annually, monthly, hourly).

The developed methods could also facilitate the decision making in developing the requirements of some building/system component (such as window properties, infiltration and ventilation rates, lighting, equipment, AHU-fans, chillers, boilers, and pumps) in the energy efficiency code for commercial buildings.

Sankey diagrams were utilized in the current work to visualize energy, mass and cost flows on various scales. The information contained in the developed Sankey diagrams would be useful in informing policy and investment decisions related to energy use patterns on the campus, building-level, and HVAC system level. Another application of Sankey diagrams is that it can help in quantifying the impact of climate change on heating and cooling loads and other building components (such as window heat gain and loss).

However, the creation process of Sankey diagrams was partially manually. Thus, a further step was taken in the second part of this work "Implementation of Sankey 
Diagrams as a Decision-Making Tool" to develop a framework to automate the process of creating Sankey diagrams from energy simulation (EnergyPlus) outputs. This framework involves the development of a user-graphical interface web page using JavaScript, Hypertext Preprocessor (PHP), and Hypertext Markup Language (HTML) to facilitate the process of creating Sankey diagrams from energy simulation outputs by users.

\section{The main contributions of this research are:}

On the campus level:

1) Developed a method to quantify and normalize resource use (per unit area and per person) obtained from meters (electricity, natural gas, steam, and water) for typical campus configuration (main power plant and various buildings varying in function and age);

2) Converting primary to secondary energy (natural gas and annual electricity mixture) including power plant efficiency and losses due to transmission and distribution (T\&D);

3) Estimating GHG emissions by different fuel types based on the average annual emission factors in Ontario for electricity, natural gas, and bio-fuel;

On the building-level:

4) Developed a workflow to convert the BIM-based architecture model to a building performance model. The workflow consists of architectural and analytical model development and model checking and conversion of BIM to gbXML data;

5) Developed a hybrid evidence-based and analytical optimization method to 
calibrate BPS models. Moreover, an inverse calibration method was developed to calibrate lighting and equipment energy consumption;

6) Converting model data to reliable energy flows and the corresponding energy cost;

On the HVAC system level:

7) Developed a method to use first principles to convert sparse AHU and plant loops data obtained from sensors and sub-meters into energy, mass, and the corresponding cost flows;

Visualization technique:

As mentioned in Section 2.5, the current visualization tools do not express the spatial and sequential nature of energy, mass, and cost flows. On the other hand, Sankey diagrams allow resource flows to be visualized within complex systems with interacting subsystems. They are particularly useful for understanding relative flows of resources and identifying opportunities for which waste outputs could be recirculated as inputs back into the system. Thus, the research:

8) Developed Sankey diagrams to visualize energy, mass, and cost flows on various scales (from campus to building system level) to help identify system inefficiencies and identify opportunities for energy savings and facilitate decision making;

9) Developed a framework to automate the process of creating Sankey diagrams from energy simulation (EnergyPlus) outputs. This workflow involves the development of a user-graphical interface web page using JavaScript, PHP, and 
HTML codes.

\subsection{Findings}

This section consists of two parts; the first part focuses on the findings from the developed methods to analyze and visualize energy and mass flows and the corresponding energy cost using Sankey diagrams on different scales (i.e. campus, building-level, and HVAC system). Moreover, this section discusses the findings from utilizing Sankey diagrams to visualize energy simulation outputs to evaluate various design alternatives. Furthermore, findings from the code developed to automate the process of creating Sankey diagrams from energy simulation outputs are discussed. The second part includes findings from the two surveys applied in this research.

By applying the proposed methods on the campus level, it was noticed that building activities/functions have a more significant impact than building age upon energy consumption and $\mathrm{CO}_{2} \mathrm{e}$ emissions. This was observed for some older buildings that were consuming less energy than newer ones having the same function/activity. Furthermore, buildings with multi-functions recorded high utility costs as they consume more amount of energy to serve different facilities such as restaurants/cafes. The GHG calculations mentioned in Section 3.3.3 was based on the annual electricity mixture - not using higher temporal resolution (i.e. hourly or daily). If the temporal resolution of this work were increased, the temporal resolution of the power generation sources would have to be incorporated.

The proposed method on the building-level helped to reveal inefficiencies of different building components and systems. For instance, the cause of highest heat loss in 
the Canal Building was through the windows ( 32 percent) during winter period. Furthermore, exfiltration and natural ventilation accounted for approximately 24 percent of heat loss from building during heating periods. While, during cooling periods, approximately 31 percent of heat gain to the building was recorded for windows. Lighting and equipment energy consumption resulted in high internal gains in both seasons. In this work, sensed and metered components (i.e. such as lights, equipment, AHU-fans, pumps, steam, chillers, and cooling tower fans) energy consumption were compared to the baseline energy consumption.

Utilizing BIM to produce energy models helped in providing details on building geometry, construction, space and zone, material properties, operational schedules, equipment and lighting power densities. Moreover, interoperability between BIM and BPS tools helped in eliminating data repetition and inherent human error. The model resolution required to perform energy analysis is based on the objective of the study. For instance, simple models are useful when estimating the amount of energy entering and leaving the building. However, more detailed models are required when detecting faults and operational problems due to the use of complex mechanical systems (i.e. offices share same VAV-boxes). In this work, a detailed model of the selected case study (the Canal Building) was required as the purpose was to provide a comprehensive energy assessment on the building-level to inform building operators with operational problems and help in visualizing unmeasured energy flows. However, some model simplification methods were implemented to reduce computation time and reduce errors (such as simplify complex geometries, exclude structural elements, lump similar windows, and merge zones having same HVAC system, setpoint and setback temperatures, schedules, internal gains, and 
boundary conditions). Figure 8.1 shows detailed and simplified energy models for the Canal building. Figure 8.2 shows a comparison between monthly measured data and calibration results of detailed and simplified energy model data for heating and cooling loads. Comparing measured data to the detailed model data, the monthly MBE and CV (RMSE) for heating loads were $-0.92 \%$ and $2.77 \%$, respectively, while $0.67 \%$ and $1.68 \%$ for cooling. Whereas comparing measured data to the simplified model data, the monthly MBE and CV (RMSE) for heating loads were $24.56 \%$ and $74.26 \%$, respectively, while $23.8 \%$ and $58.22 \%$ for cooling loads. The calibration results of the detailed model are within the ASHRAE Guideline 14-2002 thresholds. While, high discrepancy between measured data and the simplified model was recorded. For the model calibration method developed, the calibration optimization may produce mathematically correct but physically meaningless results; therefore, the user needs to countercheck results before using them. Moreover, building simulation relies on many assumptions and simplifications. Therefore, the user needs to countercheck results before using them.

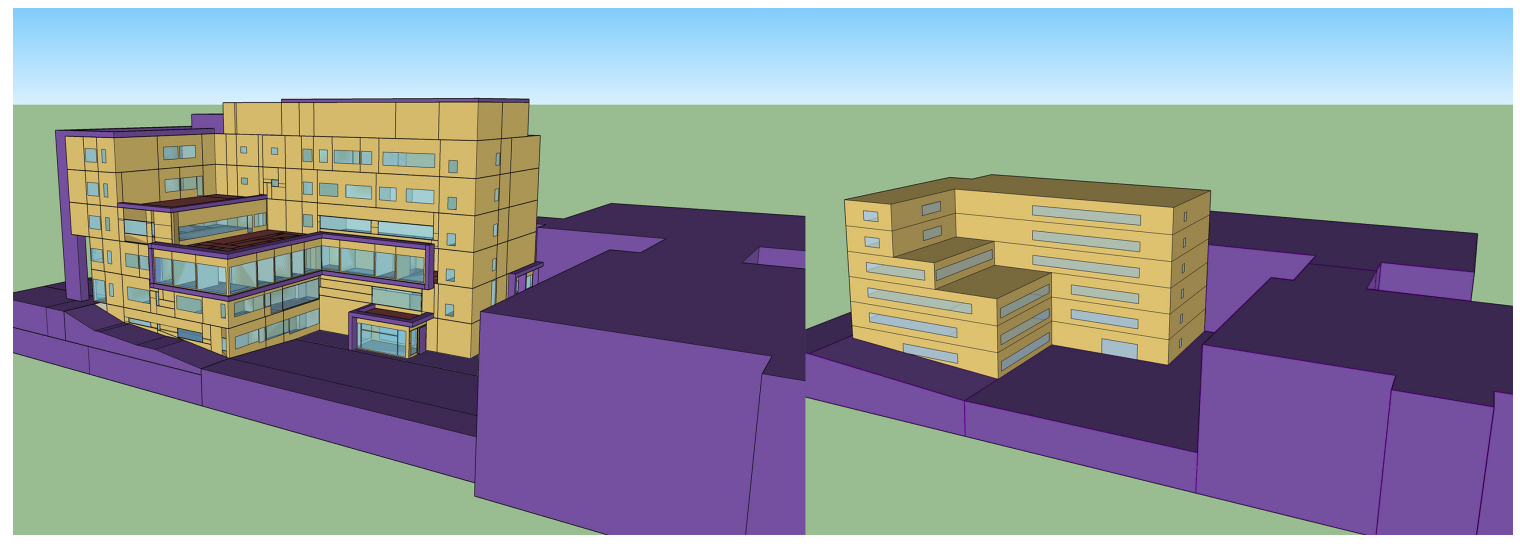

Figure 8.1: The Canal building energy model detailed (left) and simplified (right) 

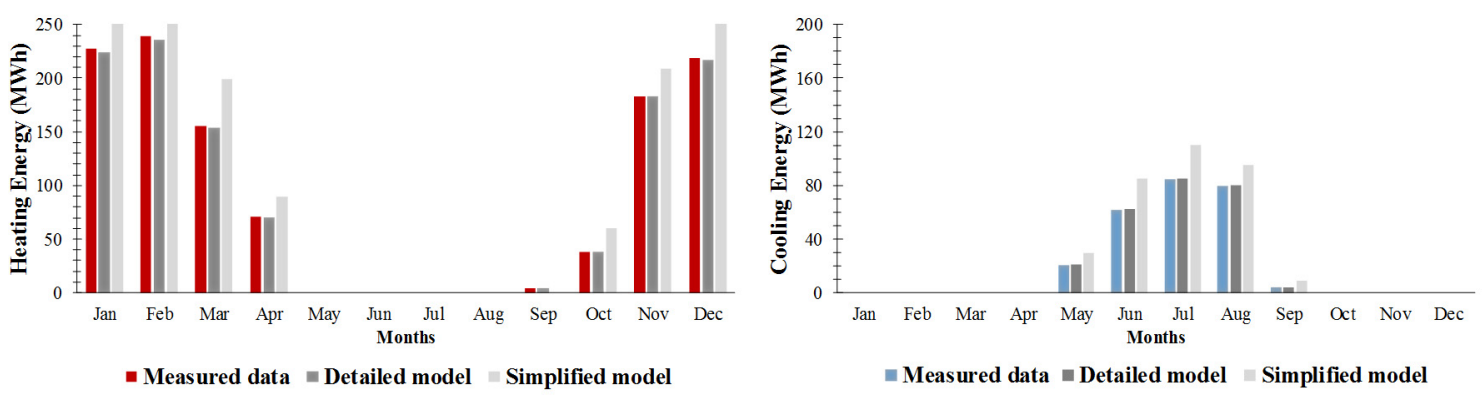

Figure 8.2: Comparison between monthly measured data and calibration results of detailed and simplified model data for heating loads (left) and cooling loads (right)

The proposed method to analyze and visualize HVAC system performance was able to provide greater understanding on how each component affects the whole system performance. Moreover, the developed Sankey diagrams helped to reveal system inefficiencies and operational problems. For instance, it was observed that the HRW operated only for 22 and 5 hours during winter and summer seasons, respectively. By checking the HRW controller, it was noticed that the allowable HRW operation temperature range (i.e. difference between sensed and setpoint temperature of the supply air) was very low. It was also observed that the supply fan energy introduced from the motor was approximately 10 percent of the total energy extracted by the cooling coils during cooling periods. Moreover, sensed and metered components (such as AHU-fans, pumps, steam, chillers, and cooling tower fans) energy consumption were compared to the baseline energy consumption. Furthermore, Sankey diagrams helped to visualize the amount of energy recirculated and exhausted in the AHU. It has been noticed that approximately 52 percent of the heat added to the supply air in the AHU was from the recirculated air during heating periods. This was due to high internal gains from equipment, lighting, occupants, and heat added by VAV-reheat coils and radiant panels installed in some of the perimeter zones. While, approximately 38 percent of the return air to the 
plenum was exhausted to the environment. The above-mentioned methods on the buildinglevel were developed on a modern commercial building. In order to implement the developed methods on an older building (e.g. masonry envelope with low metering resolution), the following steps should be considered: 1) choose the appropriate spatial (i.e. less details) and temporal (i.e. weeks or months) resolutions based on the available sensors and sub-meters, 2) an on-site audit is required to obtain information such as number of occupants, lighting and equipment power densities, and hours of operations (could be done through a survey), and 3) less information about the building requires that more parameters be optimized (such as HVAC equipment and sequence of operations) to calibrate the BPS model. However, the user needs to countercheck results before using them, as the calibration optimization may produce mathematically correct but physically meaningless results. Sankey diagrams were then applied to energy simulation outputs of a large office Canadian reference building. The proposed Sankey diagrams helped in visualizing building energy performance and to understand the upstream and downstream impact to allow various design variants to be evaluated by users. Moreover, it helped in visualizing the impact of changing building/system components to the whole system performance and demonstrate energy-saving strategies.

A framework was developed to automate the process of creating Sankey diagrams from energy simulation (EnergyPlus) outputs through a user-graphical interface web page using Hypertext Preprocessor (PHP), JavaScript, and Hypertext Markup Language (HTML) codes. The developed framework helped in: 1) generating EnergyPlus IDF files with the required list of simulation output variables and meters used to create Sankey diagrams on the building-level and HVAC system for the selected office building models 
(i.e. large, medium, and small), 2) analyzing EnergyPlus simulation output (ESO) file by matching certain strings and patterns in the IDF file, 3) generating CSV files that will be read by JavaScript code to generate Sankey diagrams, 4) check and correct energy balance across each component in the Sankey diagrams, 5) developing a user graphical interface including features (such as adding Sankey diagrams including names, uploading of IDF and ESO files, select building type, generating IDF and CSV files, fixing nodes, and color selection, 5) creating interactive Sankey diagrams (the user can select spatial and temporal resolutions from drop-down menu and slide bars, respectively), and 7) creating multi-layer Sankey diagrams to evaluate different design variants simultaneously.

Two online surveys were conducted in this research to evaluate the effectiveness and usability of utilizing Sankey diagrams to visualize energy flows on campus and building-level. As revealed from the survey results highlighted in Section 3.6, it was found that the Sankey diagrams developed on the campus level were useful in tracking underperforming buildings and to visualize overall campus performance and proportionality and providing qualitative analysis.

As revealed from the survey results highlighted in Section 4.7. The findings supported that the Sankey diagrams developed in this work helped in: 1) visualizing building-level energy flows and costs, 2) understanding the interrelated variables that affect building performance, 3) identifying system inefficiencies, 4) quantifying and understanding the impact of unmeasured energy flows, and 5) making operational problems more visible and quantifiable in order to identify opportunities for energy savings and facilitate decision making. 
There are some limitations in the usability of the proposed Sankey diagrams. For instance, they primarily provide information on the efficiency of components but do not necessarily identify poor operations. Moreover, the developed Sankey diagrams relied on historical data obtained from sensors and sub-meters and simulation outputs, thus a next step of this research is to extract the data obtained from building management system (BMS) to create Sankey diagrams that shows instantaneous energy flows on various scales.

\subsection{Future Work}

The developed methods would be useful in informing policy and investment decisions related to energy use patterns on the campus, building-level, and HVAC system level. Moreover, the developed methods could help in inspecting daily, weekly, or seasonal operation and maintenance (O\&M) issues, occupant behaviors, performance of installed equipment (e.g., HVAC and lighting), and verification of installed efficiency technologies. Moreover, the visualization technique (Sankey diagrams) developed helped in visualizing energy flows on different spatial and temporal resolutions to understand the performance of the whole system - not just components. Furthermore, Sankey diagrams helped in evaluating various design variants simultaneously in early design stage.

Future steps for this research initiative include:

- Integrating the developed methods to analyze energy and mass flows and the corresponding energy costs on campus, building-level, and HVAC system into operation and maintenance $(\mathrm{O} \& \mathrm{M})$ plan.

Carleton university started the Digital Campus Innovation (DCI) project in 2014. The aim of this project is to integrate BIM, BPS, and continuous commissioning system to 
the campus and building-level in order to provide a useful platform for building operators and other stakeholders. By integrating the developed methods in this research into $\mathrm{O} \& \mathrm{M}$ plan, this would help in inspecting operational problems and to identify opportunities for energy savings and facilitate decision making.

- Developing computer tool(s) for automating the process that creates a Sankey diagram from campus sub-meters input file.

The developed Sankey diagrams relied on historical data obtained from meters and sensors. However, real-time data analysis is more effective in order to detect operational inefficiencies and system failures. This task involves obtaining permissions to access and obtain raw data from BMS.

- Developing methods to analyze and visualize energy flows on the zone/room level using Sankey diagrams to help in inspecting fault detections. This also could facilitate greater understanding about the dynamics of energy use and occupant comfort.

This method involves the utilization of BIM and calibration of BPS models. Moreover, cardinal directions should be taken into consideration to help in understanding the occupant comfort (including thermal, indoor air quality, visual, and acoustics). Furthermore, it could help building operators and other stakeholders to make informed decisions and efficiently explore operational improvement strategies.

- Developing methods to analyze and visualize energy flows on the air distribution system (e.g., CAV and VAV boxes) using Sankey diagrams.

The current work focused on analyzing the performance of AHU and plant loops. However, future steps including analyzing and visualizing energy flows in the air distribution systems could provide greater understanding about the performance of the 
air distribution system (such as: leaking problems, performance of VAV-reheat coils, dampers operations, and optimizing air flow delivered to zones).

On the visualization side, future steps include:

- Integrating the developed framework to automate the process of creating Sankey diagrams into commercial tools (such as Revit, Insight 360, OpenStudio, etc.).

In the current work, a user-graphical interface web page was developed using PHP, HTML, and JavaScript to automate the process of creating Sankey diagrams from energy simulation outputs. Future steps include the development of a plug-in that could be integrated into commercial tools to visualize the simulation results.

- Testing the developed framework on other building systems.

The developed framework was tested on large, medium, and small reference office energy models that complies with the National Energy Code of Canada for Buildings (NECB). Future steps include the implementation of the developed code on other commercial reference buildings.

- Integrating Sankey diagrams to parametric building modelling tools (such as Dynamo Studio and Grasshopper).

The most common visualization techniques used for parameter design of buildings are 1) interactive parallel coordinates plot, 2) carpet plots, 3) voxel-plot, 4) superimposed line graph plot, 5) line chart, 6) bar graph, and 7) color mapping. However, as claimed by different authors that conveying simulation results in the form of tables and graphs is not useful to non-expert designers in the field of building science. Thus, a future step is to integrate Sankey diagrams to visualize parametric design results to enable the users to evaluate different design iterations simultaneously. 


\section{List of References}

[1] J. P. Kaye, P. M. Groffman, N. B. Grimm, L. A. Baker and R. V. Pouyat, "A Distinct Urban Biogeochemistry?," TRENDS in Ecology and Evolution, vol. 21, no. 4, pp. 192-199, 2006.

[2] C. Kennedy, J. Cuddihy and J. Engel-Yan, "The Changing Metabolism of Cities," Journal of Industrial Ecology, vol. 11, no. 2, pp. 43-59, 2007.

[3] StatCan, "Back to school... by the numbers," Statistics Canada, 5 November 2015. [Online]. Available:

http://www.statcan.gc.ca/eng/dai/smr08/2014/smr08_190_2014\#a2. [Accessed 12 February 2017].

[4] C. Klein-Banai and T. L. Theis, "An Urban University's Ecological Footprint and The Effect of Climate Change," Ecological Indicators, vol. 11, no. 3, p. 857-860, 2011.

[5] Natural Resources Canada (NRCan), "Commercial/Institutional Sector - Ontario," Natural Resources Canada (NRCan), 2016. [Online]. Available:

http://oee.nrcan.gc.ca/corporate/statistics/neud/dpa/menus/trends/comprehensive/t rends_com_on.cfm. [Accessed 5 April 2017].

[6] Environment Canada, "Greenhouse Gas Emissions by Province and Territory," 2015 a. [Online]. Available: https://www.ec.gc.ca/indicateursindicators/default.asp?lang=en\&n=18F3BB9C-1. [Accessed 01 May 2015].

[7] Environment Canada, "Government of Canada Announces 2030 Emissions Target," 2015 b. [Online]. Available: http://news.gc.ca/web/articleen.do?nid=974959. [Accessed 31 May 2015].

[8] A. Schlueter and F. Thesseling, "Building Information Model Based Energy/Exergy Performance Assessment in Early Design Stages," Automation in Construction, vol. 18, no. doi: http://dx.doi.org/10.1016/j.autcon.2008.07.003, pp. 153-163, 2009.

[9] H. J. Moon, M. S. Choi, S. K. Kim and S. H. Ryu, "Case Studies For The Evaluation of Interoperability Between A Bim Based Architectural Model And Building Performance Analysis Programs," in 12th Conference of International Building Performance Simulation Association, Sydney, 2011.

[10] K. B. Pratt and D. E. Bosworth, "A Method for The Design and Analysis of Parametric Building Energy Models," in 12th Conference of International Building Performance Simulation Association, Sydney, 2011.

[11] S. Srivastav, S. Lannon, D. K. Alexander and P. Jones, "A Review and 
Comparison of Data Visualization Techniques used in Building Design and in Building Simulation," in Eleventh International IBPSA , Glasgow, Scotland, 2009.

[12] K. Hab, S. Schweitzer, D. F. Prieto, E. Hagen, D. Engel, M. Bottinger and I. Scheler, "Visualization of Building Performance Simulation Results: State-ofThe-Art and Future Directions," in Visualization Symposium (PacificVis), DOI: 10.1109/PacificVis.2014.34, 2014.

[13] B. Lange, N. Rodriguez and W. Puech, "Energy Efficiency - A Bridge to Low Carbon Economy," in Energy Consumption Improvement Through a Visualization Software, LIRMM, Ed., France, INTECH, 2012, pp. 161-184.

[14] I. Yarbrough, Q. S. D. Reeves, K. Hackman, R. Bennett and D. Henshel, "Visualizing Building Energy Demand for Building Peak Energy Analysis," Energy and Buildings, vol. 91, no. 1 doi: http://dx.doi.org/10.1016/j.enbuild.2014.11.052, p. 10-15, 2014.

[15] F. E. M. Program, "Metering Best Practices: A Guide to Achieving Utility Resource Efficiency," Federal Energy Management Program, http://www1.eere.energy.gov/femp/pdfs/mbpg.pdf, 2007.

[16] N. S. a. T. Council, "Submetering of Building Energy and Water Usage: Analysis and Recommendations of The Subcommittee on Buildings Technology Research and Development," National Science and Technology Council, Washington, D.C., 2011.

[17] F. E. M. Program, "Guidance for Electric Metering in Federal Buildings," Federal Energy Management Program, http://www1.eere.energy.gov/femp/pdfs/adv_metering.pdf, 2006.

[18] U. E. P. A. E. STAR, "Sub-Metering Energy Use in Colleges and Universities: Incentives and Challenges," 2002.

[19] NYSERDA, "Demonstration of New Submetering Technologies," NYSERDA, Report 86-8, 1986.

[20] G. Sullivan, R. Pugh and W. Hunt, "Metering Best Practices: A Guide to Achieving Utility Resource Efficiency," http://www1.eere.energy.gov/femp/pdfs/mbpg.pdf, 2007.

[21] EPRI, End-Use Performance Monitoring Handbook, Palo Alto, California: EPRI TR-106960, Electric Power Research Institute, 1996.

[22] D. Holzer, "BIM's Seven Deadly Sins," International Journal of Architectural Computing, vol. 9, no. 4, pp. 463-480, 2011. 
[23] S. Kota, F. Stipo, W. Jeong, J. B. Kim, J. L. B. Alcocer, M. J. Clayton, W. Yan and J. S. Haberl, "Development of a Reference Building Information Model for Thermal Model Compliance Testing-Part I: Guidelines for Generating Thermal Model Input Files," ASHRAE transactions, vol. 122, pp. 256-266, 2016.

[24] C. Eastman, P. Teicholz, R. Sacks and K. Liston, BIM Handbook: A Guide to Building Information Modelling for Owners, Managers, Designers, Engineers, and Contractors, Canada: John Wiley \& Sons. Inc., 2011.

[25] J. Underwood and U. Isikdag, Handbook of Research on Building Information Modeling and Construction Informatics, IGI Global, 2012.

[26] N. Nisbet and T. Liebich, "ifcXML Implementation Guide," Building Smart, International Alliance for Interoperability (IAI), 2007.

[27] J. Plume and J. Mitchell, "Collaborative Design using a Shared IFC Building Model-Learning from Experience," Automation in Construction, vol. 16, no. 1, pp. 28-36, 2007.

[28] D. Juan and Q. Zheng, "Cloud and Open BIM-Based Building Information Interoperability Research," Journal of Service Science and Management, vol. 7, no. http://dx.doi.org/10.4236/jssm.2014.72005, pp. 47-56, 2014.

[29] D. Smith and M. Tardif, Building Information Modelling: A Strategic Implementation Guide for Architects, Engineer, Constructors, and Real Estate Asset Managers, New Jersey: John Wiley \& Sons, Inc., 2009.

[30] B. Dong, K. P. Lam and Y. C. Huang, "A Comparative Study of the IFC and gbXML Informational Infrastructures for Data Exchange in Computational Design Support Environments Geometry Information," in Building Simulation 2007. pp.1530-1537, 2007.

[31] Y. N. Bahar, C. Pere, J. Landrieu and C. Nicolle, "A Thermal Simulation Tool for Building and Its Interoperability through the Building Information Modeling (BIM) Platform," Buildings , vol. 3, no. doi:10.3390/buildings3020380, pp. 380398, 2013.

[32] Z. Shi, A. Abdelalim, . W. O’Brien, . R. Attar, P. Akik, K. Graham, B. van Waarden, S. Fai, A. Tessier and A. Khan, "Digital Campus Innovation Project: Integration of Building Information Modeling with Building Performance Simulation and Building Diagnostics," Washington, 2015.

[33] DOE, "EnergyPlus Energy Simulation Software," U.S. Department of Energy, 2009. [Online]. Available: http://apps1.eere.energy.gov/buildings/energyplus/. [Accessed 9 September 2015]. 
[34] T. Kusuda, "Early History and Future Prospects of Buildings System Simulation," in Proceedings of the 6th International Building Simulation IBPSA conference, Kyoto, Japan, 1999.

[35] J. Clarke, Energy Simulation in Building Design (Second Edition), the University of Michigan: A. Hilger ISBN: 978-0-7506-5082-3, 1985.

[36] J. Lebrun and G. Liebecq, "Energy Conservation in Buildings and Community Systems Annex 10: Building HVAC System Simulation," International Energy Agency, Synthesis Report, 1988.

[37] D. B. Crawley, J. W. Hand, M. Kummert and B. T. Griffith, "Contrasting The Capabilities of Building Energy Performance Simulation Programs," Building and Environment, vol. 43, no. 4, pp. 661-673, 2008.

[38] J. Visier and M. Jandon, "Energy Conservation in Buildings and Community Systems Annex 40: Commissioning of Building HVAC Systems for Improved Energy Performances," International Energy Agency, Synthesis Report, 2004.

[39] R. Jagpal,, "Energy Conservation in Buildings and Community Systems Annex 34 : Computer Aided Evaluation of HVAC System Performance," International Energy Agency, Synthesis Report, 2006.

[40] M. Krarti, Energy Audit of Building Systems; An Engineering Approach, Florida, U.S.: CRC Press, 2000.

[41] J. Spitler, "Building performance simulation: the now and the not yet," $H V A C \& R$ Research, vol. 12, no. 3a, pp. 711-713, 2006.

[42] J. Lebrun and S. Wang, "Energy Conservation in Buildings and Community Systems Annex 17: Building Energy Management Systems - Evaluation and Emulation Techniques," International Energy Agency, Synthesis Report, 1993.

[43] M. Fels, "Prism: An Introduction," Energy and Buildings, vol. 9, pp. 5-18, 1986.

[44] J. Kissock, J. Haberl and D. Claridge, "Development of a Toolkit for Calculating Linear, Change-point Linear and Multiple-linear Inverse Building Energy Analysis Models," Final Report, ASHRAE 1050-RP, 2002.

[45] T. Reddy, S. Deng and D. Claridge, "Development of An Inverse Method to Estimate Overall Building and Ventilation Parameters of Large Commercial Buildings," Journal of Solar Energy Engineering, vol. 121, pp. 40-46, 1999.

[46] LBNL, "DOE-2 User Guide, Ver. 2.1.," Lawrence Berkeley Laboratory and Los Alamos National Laboratory, LBL Report No. LBL-8689 Rev. 2, Berkeley, CA, 1980 . 
[47] S. Klein, "TRNSYS 16 Program Manual," Solar Energy Laboratory, University of Wisconsin, Madison, USA, 2007.

[48] W. Carroll and R. Hitchcock, "Tuning Simulated Building Descriptions to Match Actual Utility data: Methods AND Implementation," ASHRAE Transactions, vol. 99, pp. 928-934, 1993.

[49] M. Kaplan, J. McFerran, J. Jansen and R. Pratt, "Reconciliation of a DOE2.1c Model with Monitored End-use Data for a Small Office Building," ASHRAE Transactions, pp. 981-993, 1990.

[50] T. Reddy and I. Maor, "Procedures for Reconciling Computer-Calculated Results With Measured Energy Data," ASHRAE Research Project 1051-RP, Atlanta, 2006.

[51] T. Reddy, "Literature Review on Calibration of Building Energy Simulation Programs: Uses,Problems, Procedures, Uncertainty and Tools," ASHRAE Transactions, vol. 112, no. 1, pp. 226-240, 2006.

[52] K. Subbarao, "PSTAR - Primary and Secondary Terms Analysis and Renormalization. A Unified Approach to Building Energy Simulations and ShortTerm Monitoring," SERI/TR-254-3175, Solar Energy Research Institute, Golden, CO, 1988b.

[53] Y. Pan, Z. Huang and G. Wu, "Calibrated Building Energy Simulation and Its Application In a Highrise Commercial Building in Shanghai," Energy and Buildings, vol. 39, pp. 651-657, 2007.

[54] A. Pedrini, F. Westphal and R. Lamberts, "A Methodology for Building Energy Modeling and Calibration in Warm Climates," Building and Environment, vol. 37, pp. 903-912, 2002.

[55] F. Westphal and R. Lamberts, "Building Simulation Calibration using Sensitivity Analysis," in Proceedings of the 9th IBPSA Building Simulation Conference, Montréal, Canada, 2005.

[56] J. Yoon, J. Lee and D. Claridge, "Calibration Procedure for Energy Performance Simulation of A Commercial Building," Journal of Solar Energy Engineering, vol. 125, pp. 251-257, 2003.

[57] P. Raftery, M. Keane and A. Costa, "Calibrating Whole Building Energy Models: Detailed Case Study Using Hourly Measured Data," Energy and Buildings, vol. 43, no. 12, pp. 3666-3679, 2011.

[58] A. Costa, M. Keane,, P. Raftery and J. O’Donnell, "Key factors - Methodology for Enhancement and Support of Building Energy Performance," in Proceedings 
of the 11th IBPSA Conference, Glasgow, UK, 2009.

[59] P. Raftery, M. Keane and A. Costa, "Calibration of A Detailed Simulation Model To Energy Monitoring System Data: A Methodology And Case Study," Glasgow, Scotland, 2009.

[60] A. E. Kandil and J. A. Love, "Signature Analysis Calibration of A School Energy Model Using Hourly Data," Journal of Building Performance Simulation, vol. doi: 10.1080/19401493.2013.838608, pp. 1-20, 2013.

[61] K. P. Lam, J. Zhao, E. B. Ydstie, J. Wirick, M. Qi and J. Park, "An Energyplus Whole Building Energy Model Calibration Method For Office Buildings Using Occupant Behavior Data Mining And Empirical Data," in ASHRAE/IBPSA-USA Building Simulation Conference, Atlanta, GA, 2014.

[62] T. A. Reddy, I. Maor and C. Panjapornpon, "Calibrating Detailed Building Energy Simulation Programs with Measured Data-Part I: General Methodology (RP1051)," HVAC\&R Research, vol. 13, no. 2, pp. 221-241, 2007.

[63] R. Oliva, "Model Calibration as a Testing Strategy for System Dynamics Models," European Journal of Operational Research, vol. 151, no. 5, pp. 52-68, 2003.

[64] J. Haberl and T. Bou-Saada, "Procedures for Calibrating Hourly Simulation Models to Measured Building Energy and Environmental Data," J. Sol. Energy Eng., vol. 120, pp. 193-204, 1998.

[65] M. Liu, D. Claridge, N. Bensouda, K. Heinemeier, S. Lee and G. Wei, "Manual of Procedures for Calibrating Simulations of Building Systems," HPCBS\#E5P23T2b. California Energy Commission, Public Interest Energy Research Program, California, 2003.

[66] T. Bou-Saada and J. Haberl, "An Improved Procedure for Developing Calibrated Hourly Simulation Models," in Proceedings of the 5th IBPSA Building Simulation Conference, Madison, Wisconsin, USA, 1995.

[67] J. McCray, P. Bailey and J. Parker, "Using Data Visualization Tools for The Calibration of Hourly DOE-2.1 Simulations," in Proceedings of the 5th IBPSA Building Simulation Conference, Madison, Wisconsin, USA, 1995.

[68] G. Wei, M. Liu and D. Claridge, "Signatures of Heating and Cooling Energy Consumption for Typical AHUs," in Proceedings of the Eleventh Symposium on Improving Building Systems in Hot and Humid Climates, Fort Worth, TX, 1998.

[69] M. Liu, "User's Manual for Air Side Simulation Programs (AirModel)," Energy Systems Laboratory, Texas A\&M University, College Station, TX, 1997. 
[70] M. Liu, L. Song, G. Wei and D. Claridge, "Simplified Building and Air-Handling Unit Model Calibration and Applications," Journal of Solar Energy Engineering, vol. 126, pp. 601-609, 2004.

[71] S. Bertagnolio, "Evidence-Based Model Calibration For Efficient Building Energy Services," Energy Systems Research Unit Aerospace And Mechanical Engineering Department Faculty of Applied Sciences University of Liège In Partial Fulfillment of the requirements for the degree of doctor of applied sciences, Wallonia, Belgium, 2012.

[72] E. Fabrizio and V. Monetti, "Methodologies and Advancements in the Calibration of Building Energy Models," energies, vol. 8, no. ISSN 1996-1073, pp. 25482574, 2015.

[73] D. Coakley, P. Raftery and M. Keane, "A Review of Methods to Match Building Energy Simulation Models to Measured Data," Renew. Sustain. Energy Rev., vol. 37, pp. 123-141, 2014.

[74] Y. Heo, "Bayesian Calibration of Building Energy Models for Energy Retrofit Decision-Making Under Uncertainty," PhD Thesis, Georgia Institute of Technology, Georgia, 2011.

[75] A. Booth, R. Choudhary and D. Spiegelhalter, "Handling Uncertainty in Housing Stock Models," Build. Environ., vol. 48, pp. 35-47, 2012.

[76] Y. Heo, R. Choudhary and G. Augenbroe, "Calibration of Building Energy Models for Retrofit Analysis Under Uncertainty," Energy Build, vol. 47, pp. 550560, 2012.

[77] M. Manfren, N. Aste and R. Moshksar, "Calibration and Uncertainty Analysis for Computer Models-A Meta-Model Based Approach for Integrated Building Energy Simulation," Appl. Energy, vol. 103, pp. 627-641, 2013.

[78] G. Pavlak, A. Florita, G. Henze and B. Rajagopalan, "Comparison of Traditional and Bayesian Calibration Techniques for Gray-Box Modeling," J. Arch. Eng., pp. doi:10.1061/(ASCE)AE.1943-5568.0000145, 2014.

[79] A. Saltelli, S. Tarantola and F. Campolongo, "Sensitivity Analysis as An Ingredient of Modeling," Stat. Sci., vol. 15, pp. 377-395, 2000.

[80] L. Van Gelder, P. Das, H. Janssen and S. Roels, "Comparative Study of Metamodelling Techniques in Building Energy Simulation: Guidelines for Practitioners," Simul. Model. Pract. Theory, vol. 49, pp. 245-257, 2014.

[81] B. Eisenhower, Z. O'Neill, S. Narayanan, V. Fonoberov and I. Mezic, "A Methodology for Meta-Model Based Optimization in Building Energy Models," 
Energy Build, vol. 47, pp. 292-301, 2012.

[82] A. Nguyen, S. Reiter and P. Rigo, "A Review on Simulation-Based Optimization Methods Applied to Building Performance Analysis," Appl. Energy, vol. 113, pp. 1043-1058, 2014.

[83] R. Evins, "A Review of Computational Optimization Methods Applied to Sustainable Building Design," Renew. Sustain. Energy Rev, vol. 22, pp. 230-245, 2013.

[84] V. Machairasetal, A. Tsangrassoulis and K. Axarli, "Algorithms for Optimization of Building Design: A Review," Renew. Sustain. Energy Rev, vol. 31, pp. 101$112,2014$.

[85] V. Norrefeldt, G. Grün and C. van Treeck, , "Use of the VEPZO model to optimize a hybrid ventilation system," in (BauSIM 2012): Fourth GermanAustrian IBPSA Conference Berlin University of the Arts, Berlin, Germany, 2012.

[86] S. Liu and G. Henze, "Calibration of Building Models for Supervisory Control of Commercial Building," in Proceedings of the Ninth International IBPSA Conference 2005, Montréal, QC, Canada, 15-18, 2005.

[87] A. Hani and T. Koiv, "Optimization of Office Building Façades in A Warm Summer Continental Climate," Smart Grid Renew. Energy, vol. 3, pp. 222-230, 2012.

[88] M. Ferrara, E. Fabrizio, J. Virgone and M. Filippi, "A Simulation-Based Optimization Method for Cost-Optimal Analysis of Nearly Zero Energy Buildings," Energy Build, vol. 84, pp. 442-457, 2014.

[89] S. U. Lee and D. Claridge, "Automatic Calibration of a Building Energy Simulation Model Using a Global Optimization Program," Texas A\&M University (http://www.tamu.edu); Energy Systems Laboratory (http://esl.tamu.edu). Available electronically from http : / /hdl .handle .net /1969 $.1 / 5186$, Texas, 2002.

[90] LBNL, "GenOpt," Lawrence Berkeley National Laboratory, Berkeley, CA, 2011.

[91] K. Lavigne, "Assisted Calibration in Building Simulation-Algorithm Description and Case Studies," in Proceedings of the 11th IBPSA Conference, Glasgow, UK, 2009.

[92] J. Sanyal, J. New, R. E. Edwards and L. Parker, "Calibrating building energy models using supercomputer trained machine learning agents," Concurrency and Computation: Practice and Experience, vol. 26, no. 13, pp. 2122-2133, 2013. 
[93] D. Coakley, P. Raftery, P. Molloy and G. White, "Calibration of A Detailed Bes Model To Measured Data Using An Evidence-Based Analytical Optimisation Approach," in Proceedings of Building Simulation 2011: 12th Conference of International Building Performance Simulation Association, Sydney, 2011.

[94] J. Sun and T. A. Reddy, "Calibration of Building Energy Simulation Programs Using the Analytic Optimization Approach (RP-1051)," HVAC\&R Research, vol. 12, no. 1, pp. 177-196, 2006.

[95] M. Taheri, F. Tahmasebi and A. Mahdavi, "A Case Study of Optimization-Aided Thermal Building Performance Simulation Calibration," in Proceedings of the 13th Conference of International Building Performance Simulation Association, Chambéry, France, 25-28, 2013.

[96] F. Campolongo, A. Saltelli and J. Cariboni, , "From Screening to Quantitative Analysis. A Unified Approach," Comput. Phys. Commun., vol. 182, pp. 978-988, 2011.

[97] ASHRAE, "ASHRAE Guideline: Measurement of Energy and Demand Savings," ASHRAE Guideline 14-2002, 2002.

[98] IPMVP, "International Performance Measurement \& Verification Protocol: Concepts and Options for Determining Energy and Water Savings," Available electronically at http://www.doe.gov/bridge, Oak Ridge, TN, 2002.

[99] FEMP, "M\&V Guidelines: Measurement and Verification for Federal Energy Projects," U.S. DEPARTMENT OF ENERGY OFFICE OF ENERGY EFFICIENCY AND RENEWABLE ENERGY, Berkeley, 2000.

[100] J. Waltz, Computerized Building Energy Simulation Handbook, Lilburn, GA: Fraimont Press, 2000.

[101] M. Schmidt, "The Sankey Diagram in Energy and Material Flow Management (Part II:Methodology and Current Applications)," Journal of industrial ecology, vol. 12, no. 2, pp. 173-185, 2008.

[102] IEA, NComVA, 2015. [Online]. Available: http://www.iea.org/sankey/\#?c=World\&s=Balance. [Accessed 15 September 2015].

[103] Canadian Energy Systems Analysis Research (CESAR), "Sankey diagrams of Canada's energy systems," Canadian Energy Systems Analysis Research (CESAR), 2016. [Online]. Available: http://www.cesarnet.ca/visualization/sankey-diagrams-canadas-energy-systems. [Accessed 29 January 2017]. 
[104] N. Gershon, S. Eick and S. Card, "Information Visualization," Interactions, vol. 5, no. 2, pp. 9-15, 1998.

[105] S. Card, J. Mackinlay and B. Shneiderman, Readings in Information Visualization: Using Vision to Think, Morgan Kaufmann, 1999.

[106] S. Meyers, E. Mills, A. Chen and L. Demsetz, "Building Data Visualization for Diagnostics, Operator Feedback, and Performance Optimization," ASHRAE Journal, no. June, pp. 63-73, 1996.

[107] J. Scholtz, "Beyond Usability: Evaluation Aspects of Visualanalytic Environments," in IEEE Symposium on Visual Analytics Science and Technology, Richland, WA, 2006.

[108] C. Plaisant, J. D. Fekete and G. Grinstein, "Promoting Insight-Based Evaluation of Visualizations: From Contest to Benchmark Repository," IEEE Transactions on Visualization and Computer Graphics, vol. 14, pp. 120-134, 2008 a.

[109] C. Plaisant, G. Grinstein, J. Scholtz, M. Whiting, T. O’Connell, S. Laskowski, L. Chien, A. Tat, W. Wright, C. Gorg, Z. Liu, N. Parekh, K. Singhal and J. Stasko, "Evaluating Visual Analytics at The 2007 Vast Symposium Contest," IEEE Computer Graphics and Applications, vol. 28, pp. 12-21, 2008b.

[110] S. Greenberg and B. Buxton, "Usability Evaluation Considered Harmful (some of the time)," in Proceedings of the twenty-sixth annual SIGCHI conference on Human factors in computing systems, ACM, New York,, 2008.

[111] J. Ferwerda, H. Rushmeier and B. Watson, "Frontiers in perceptually-based image synthesis: modeling, rendering, display, validation. Half day tutorial," ACM SIGGRAPH 2003 conference, San Diego, CA, July, 2003.

[112] S. Laskowski and C. Plaisant, "Evaluation Methodologies for Visual Analytics," in Illuminating the Path: The Research and Development Agenda for Visual Analytics, Los Alamitos, CA, J. J. Thomas and K. A. Cook, Eds. IEEE Computer Society, 2005, pp. 150-157.

[113] C. North, "Toward measuring insight," IEEE Computer Graphics and Applications, vol. 26, no. 1, pp. 6-9, 2006.

[114] M. Asl, M. Bergin, A. Menter and W. Yan, "BIM-based parametric building energy performance multi-objective optimization," Education and Research in Computer Aided Architectural Design in Europe, vol. 32, pp. 1-10, 2014.

[115] E. Achtert, H.-P. Kriegel and E. Schubert, "Interactive Data Mining with 3DParallel-Coordinate-Trees," New York City, NY. doi:10.1145/2463676.2463696, 2013. 
[116] P. Raftery and M. Keane, "Visualizing Patterns in Building Performance Data," in 12th Conference of International Building Performance Simulation Association, Sydney, 2011.

[117] A. Telea, Data Visualization: Principles and Practice, Second Edition, 2 ed., ISBN 9781498759793: CRC Press, 2015.

[118] A. Wolman, "The metabolism of cities," in The weight of nations: Material outflows from industrial economies, Washington, DC, Scientific American, 1965, pp. 178-193.

[119] E. H. Decker, S. Elliott, F. A. Smith, D. R. Blake and F. S. Rowland, "Energy and Material Flow Through The Urban Ecosystem," Annual Review of Energy and the Environment, vol. 25, no. 1, pp. 685-740, 2000.

[120] H. Girardet, W. N'Dow, J. G. Wood and D. Rees, The Gaia Atlas of Cities: New Directions for Sustainable Urban Living, II ed., New York, US: Gaia Books Ltd; New edition edition, 1996.

[121] H. R. Sahely, S. Dudding and C. A. Kennedy, "Estimating The Urban Metabolism of Canadian Cities: Greater Toronto Area Case Study," Canadian Journal of Civil Engineering, vol. 30, no. 2, pp. 468-483, 2003.

[122] H. Tisha and S. Pincetl, "Urban Metabolism Literature Review," UCLA Institute of the Environment, Los Angeles, 2012.

[123] C. Kennedy, S. Pincetl and P. Bunje, "The study of urban metabolism and its applications to urban planning and design," Environmental Pollution, vol. 159, no. 8-9, p. 1965-1973, 2011.

[124] N. Codoban and C. Kennedy, "Metabolism of Neighborhoods," Journal of Urban Planning, vol. 134, pp. 21-31, 2008.

[125] S. Li, Y. Zhang, Z. Yang and J. Zhang, "Ecological Relationship Analysis of The Urban Metabolic System of Beijing, China," Environmental Pollution, vol. 170, pp. 169-76, 2012.

[126] S. Barles, "Urban Metabolism of Paris And Its Region," Journal of Industrial Ecology, vol. 13, pp. 898-913, 2009.

[127] C. Kennedy, I. D. Stewart, N. Ibrahim, A. Facchini and R. Mele, "Developing a multi-layered indicator set for urban metabolism studies in megacities," Ecological Indicators, vol. 47, pp. 7-15, 2014.

[128] B. Goldstein, M. Birkved, M.-B. Quitzau and M. Hauschild, "Quantification of Urban Metabolism Through Coupling With The Life Cycle Assessment Framework: Concept Development And Case Study," Environmental Research 
Letters, Vols. http://dx.doi.org/10.1088/1748-9326/8/3/035024, 2013.

[129] R. Obernosterer, P. H. Brunner, H. Daxbeck, T. Gagan, E. Glenck, C. Hendriks and I. Reiner, "Materials Accounting As A Tool Fordecision Making In Environmentalpolicy (Mac Tempo)," Institute for Water Quality and Waste Management, Vienna University, Austria, 1998.

[130] C. Hendriks, R. Obernosterer, D. Müller, S. Kytzia, P. Baccini and P. H. Brunner, "Material Flow Analysis: A Tool To Support Environmental Policy Decision Making. Case-Studies On The City of Vienna and The Swiss Lowlands," Local Environment: The International Journal of Justice and Sustainability, vol. 5, no. 3, pp. 311-328, 2000.

[131] L. Talmon-Gros, Development Patterns of Material Productivity: Convergence or Divergence?, 1 ed., Switzerland: Springer Science \& Business Media, 2014.

[132] M. M. Khasreen, P. F. Banfill and G. F. Menzies, "Life-Cycle Assessment and the Environmental Impact of Buildings: A Review," Sustainability, vol. 1, pp. 674701, 2009.

[133] J. Norman, H. L. MacLean and C. A. Kennedy, "Comparing High and Low Residential Density: Life-Cycle Analysis of Energy Use and Greenhouse Gas Emissions," Journal of Urban Planning and Development, vol. 132, no. 1, pp. 10$21,2006$.

[134] EPA, "Life-Cycle Assessment," 2014 a. [Online]. Available: http://www.epa.gov/sustainability/analytics/life-cycle.htm. [Accessed 09 June 205].

[135] T. Ramesh, R. Prakash and K. Shukla, "Life Cycle Energy Analysis of Buildings: An Overview," Energy and Buildings, vol. 2, p. 1592-1600, 2010.

[136] S. Pincetl , P. Bunje and T. Holmes, "An Expanded Urban Metabolism Method: Towards A Systems Approach For Assessing The Urban Energy Processes And Causes," Landscape and Urban Planning, pp. 193-202, 2012.

[137] M. Chester, S. Pincetl and B. Allenby, "Avoiding Unintended Tradeoffs By Integrating Life-Cycle Impact Assessment With Urban Metabolism," Current Opinion in Environmental Sustainability, vol. 4, no. 4, pp. 451-457, 2012.

[138] T. M. Conway, C. Dalton, J. Loo and L. Benakoun, "Developing Ecological Footprint Scenarios On University Campuses: A Case Study of The University of Toronto At Mississauga," International Journal of Sustainability in Higher Education, vol. 9, no. 1, pp. 4-20, 2008.

[139] G. F. Dawe, A. Vetter and S. Martin , "An Overview of Ecological Footprinting and Other Tools and Their Application To The Development of Sustainability 
Process: Audit and Methodology At Holme Lacy College, Uk," International Journal of Sustainability in Higher Education, vol. 5, no. 4, pp. 340-371, 2004.

[140] K. Flint, "Institutional Ecological Footprint Analysis - A Case Study of The University of Newcastle, Australia," International Journal of Sustainability in Higher Education, vol. 2, no. 1, pp. 48 - 62, 2001.

[141] EPA, "Environmental Footprint Analysis," 2014 b. [Online]. Available: http://www.epa.gov/sustainability/analytics/environmental-footprint.htm. [Accessed 06 June 2015].

[142] McGill University, "McGill Energy Dashboard," 2015. [Online]. Available: https://my.pulseenergy.com/mcgill/dashboard\#/location/1585. [Accessed 26 February 2015].

[143] UBC, "UBC Energy Demand," 2014 a. [Online]. Available: https://my.pulseenergy.com/ubc/dashboard\#/overview. [Accessed 26 Febrarury 2015].

[144] Arizona State University (ASU), "ASU Campus Metabolism," 2015. [Online]. Available: http://cm.asu.edu/. [Accessed 31 May 2015].

[145] Lucid, "Building Dashboard," 2014. [Online]. Available: http://www.luciddesigngroup.com/buildingdashboard/index.html. [Accessed 24 February 2015].

[146] BuildingDashboard, "BuildingDashboard: Carleton University," 2014. [Online]. Available: http://buildingdashboard.net/carletonu/\#/carletonu. [Accessed 25 June 2015].

[147] SCP, "Sustainable Campus Program," 2014. [Online]. Available: http://frisco.ahec.edu/\#/home/. [Accessed 20 Febraury 2015].

[148] B. Howard, L. Parshall, J. Thompson, S. Hammer, J. Dickinson and V. Modi, "Spatial Distribution of Urban Building Energy Consumption By End Use," Energy and Buildings, vol. 45, pp. 141-151, 2012.

[149] Y. Agarwal, T. Weng and R. K. Gupta, "The energy dashboard: improving the visibility of energy consumption at a campus-wide scale," New York, NY, USA, 2009.

[150] K. McCusker, "Measuring, Managing and Visualizing Building Energy Consumption \& Carbon Emissions: Benchmarking at the University of Massachusetts Amherst," Amherst, 2013.

[151] W. O'Brien, "Preliminary Investigation of The Use of Sankey Diagrams to Enhance Building Performance Simulation-Supported Design," in Symposium on 
Simulation for Architecture and Urban Design. Society for Computer Simulation International, 2012.

[152] S. L. Singer and A. J. Simon, "EEBHub Navy Yard Sankey Diagram Energy Analysis Final Report," Lawrence Livermore National Laboratory, Tech. Rep. LLNLTR-613594, CA, 2013.

[153] R. Sims, R. Schock, A. Adegbululgbe, J. Fenhann, I. Konstantinaviciute, W. Moomaw, H. Nimir, B. Schlamadinger, J. Torres-Martínez, C. Turner, Y. Uchiyama, S. Vuori, N. Wamukonya and X. Zhang, "Energy supply," in In Climate Change 2007: Mitigation, B. Metz, O. Davidson, P. Bosch, R. Dave and L. Meyer, Eds., Cambridge, United Kingdom and New York, NY, USA, Cambridge University Press, 2007, pp. 253-322.

[154] F. Heidt, "CASAnova," 2012. [Online]. Available: http://nesal.unisiegen.de/index.htm?/softlab/casanova_e.htm. [Accessed 20 February 2015].

[155] Sefaira, "Sefaira," 2012. [Online]. Available: http://sefaira.com/our-resources/. [Accessed 18 February 2015].

[156] L. E. Fedoruk, R. J. Cole, J. B. Robinson and A. Cayuela , "Learning from failure: understanding the anticipated-achieved building energy performance gap," Building Research \& Information, p. DOI:10.1080/09613218.2015.1036227, 2015.

[157] Cleaver-Brooks, "Boiler Efficiency Guide," Cleaver-Brooks, Inc, Thomasville, 2010.

[158] International Energy Agency (IEA)/OECD/eurostat, "Energy Statistics Manual," International Energy Agency (IEA), 2005.

[159] M. Deru and P. Torcellini , "Source Energy and Emission Factors for Energy Use in Buildings," National Renewable Energy Laboratory, Colorado, 2007.

[160] K. Ueno and J. Straube, "BSD-151:Understanding Primary/Source and Site Energy," Building Science Press, 2010.

[161] AMPCO, "Electricity in Ontario," (AMPCO) Association of Major Power Consumers in Ontario, Toronto, 2011.

[162] Energy Star, "Source Energy," Energy Star Portfolio Manager, Pennsylvania, 2013.

[163] ieso, "Ontario's Electricity Hub," 2015. [Online]. Available: http://www.ieso.ca/Pages/Power-Data/supply.aspx. [Accessed 23 May 2015]. 
[164] CANSIM, "Electricity Generated from Fuels, by Electric Utility Thermal Plants," 2014 a. [Online]. Available:

http://www5.statcan.gc.ca/cansim/a26?lang=eng\&retrLang=eng\&id=1270006\&ta bMode $=$ dataTable\&srchLan=-1\&p1=-1\&p2=9. [Accessed 01 June 2015].

[165] CANSIM, "Fuel Consumed for Electric Power Generation, by Electric Utility Thermal Plants," 2014 b. [Online]. Available:

http://www5.statcan.gc.ca/cansim/a26?lang=eng\&retrLang=eng\&id=1270004\&ta bMode $=$ dataTable $\&$ srchLan $=-1 \& p 1=-1 \& p 2=9$. [Accessed 01 June 2015].

[166] ers, "Independent Audit of Enbridge Gas Distribution 2012 DSM Program Results," (ers) Energy \& Resource Solution, North Andover, 2013.

[167] L. Honorio, J.-G. Bartaire, R. BAUERSCHMIDT, T. OHMAN, Z. TIHANYI, H. ZEINHOFER, J. F. SCOWCROFT, V. DE JANEIRO, H. KRUGER, H.-J. MEIER, D. OFFERMANN and U. LANGNICKEL, "Efficiency in Electricity Generation," Union of the Electricity Industry - EURELECTRIC, VGB, Brussels, 2003.

[168] ieso, "Ontario’s Electricity System," 2014. [Online]. Available: http://www.ieso.ca/ontarioenergymap/index.html. [Accessed 18 May 2015].

[169] C. Maas, "Greenhouse Gas and Energy Co-Benefits of Water Conservation," POLIS Research Report 09-01, Victoria, 2009.

[170] C. Maas, "Ontario's Water-Energy Nexus: Will We Find Ourselves in Hot Water... or Tap into Opportunity?," POLIS Project on Ecological Governance, Victoria, 2010.

[171] OSSTF, "Ontario Secondary Svhool Teachers' Federation: Occupancy and Load Capacity," 2015. [Online]. Available: http://www.osstf.on.ca/en$\mathrm{CA} /$ services/health-safety/information-bulletins/occupancy-and-load-capacity. [Accessed 19 May 2015].

[172] J. SKOPEK, "Factors Affecting Building Performance," Prague, 2013.

[173] M. S. Gul and S. Patidar, "Understanding The Energy Consumption And Occupancy of A Multi-Purpose Academic Building," Energy and Buildings, vol. 87, p. 155-165, 2015.

[174] EPA, "Overview of Greenhouse Gases," 2015. [Online]. Available: http://www.epa.gov/climatechange/ghgemissions/gases.html. [Accessed 25 June 2015].

[175] E. Kikuchi, D. Bristow and C. A. Kennedy, "Evaluation of region-specific residential energy systems for GHG reductions: Case studies in Canadian cities," 
Energy Policy, vol. 37, no. 4, p. 1257-1266, 2009.

[176] T. Nitheanandan and M. Brown, "A HistoricaL Review of Nuclear Contribution To The Ontario Energy Mix," Vancouver, 2014.

[177] Natural Resources Canada (NRCan), "CO2 Emission Factors," 2013 b. [Online]. Available: http://www.nrcan.gc.ca/energy/efficiency/industry/technicalinfo/benchmarking/canadian-steel-industry/5193\#archived. [Accessed 05 March 2015].

[178] E. Canada, "Canada's Emissions Trends," Environment Canada , ISSN 22919392, 2013.

[179] Natural Resources Canada (NRCan), "Canadian Climate Normals 1981-2010 Station Data," 2015. [Online]. Available:

http://climate.weather.gc.ca/climate_normals/results_1981_2010_e.html?stnID=4 $337 \&$ lang $=\mathrm{e} \& \mathrm{dCode}=1 \&$ province $=\overline{\mathrm{O}} \mathrm{NT} \& \operatorname{provBut}=\overline{\mathrm{Go}} \& \operatorname{mon} \mathrm{th} 1=0 \&$ month $2=12$. [Accessed 01 March 2015].

[180] Honeywell, "Carleton University Energy Master Plan," Honeywell Energy and Environmental Solutions, Markham, 2014.

[181] Carleton University, "Facts and Figures Carleton at a Glance," 2015. [Online]. Available: http://carleton.ca/about/facts/. [Accessed 19 May 2015].

[182] R. Wood, "List of Sports - Every Sport," 2008. [Online]. Available: http://www.topendsports.com/sport/sport-list.htm. [Accessed 24 May 2015].

[183] Google Developers, "Google Developers Charts: Sankey Diagram," Google Developers, 30 June 2015. [Online]. Available: https://developers.google.com/chart/interactive/docs/gallery/sankey. [Accessed 01 July 2015].

[184] Renewables Academy (renac), "CHP - Cogeneration Power," http://www.erab.com/skiss/uk68.pdf, Berlin, Germany, 2013.

[185] UBC, "Academic District Energy System - Steam To Hot Water Conversion Project," 2014 b. [Online]. Available: http://www.projectservices.ubc.ca/portfolio/alternative-energy/hot-waterloop.htm. [Accessed 03 June 2015].

[186] Carleton University, "University Buildings Carleton Campus: The H.H.J. Nesbitt Biology Building," 2014. [Online]. Available: http://carleton.ca/campus/buildings/the-h-h-j-nesbitt-biology-building/. [Accessed 10 June 2015]. 
[187] L. K. Murugesan, R. Hoda and Z. Salcic, "Visualization of Electricity Consumption: Software Prototype through Literature Survey," International Journal of Innovative Research in Science, Engineering and Technology, vol. 3, no. 3 (ISSN (Online): 2319-8753), pp. 2725-2729, 2014.

[188] G. Stavropoulos, S. Krinidis, D. Ioannidis, K. Moustakas and D. Tzovaras, "A Building Performance Evaluation \& Visualization System," in IEEE International Conference on Big Data, 2014.

[189] D. Claridge, "Building Simulation for Practical Operational Optmization," in Building Performance Simulation for Design and Operation, Oxfordshire, UK, 1st ed.; Hensen, J.L.M., Lamberts, R., Eds.; Spon Press, 2011.

[190] M. Emily, E. Ryan and T. Sanquist, "Validation of Building Energy Modeling Tools Under Idealized and Realistic Conditions," Energy Build, vol. 47, pp. 375382, 2012.

[191] A. S. Silva and E. Ghisi, "Uncertainty Analysis of User Behaviour and Physical Parameters in Residential Building Performance Simulation," Energy and Buildings, vol. 76, no. doi: http://dx.doi.org/10.1016/j.enbuild.2014.03.001, pp. 381-391, 2014.

[192] G. Dall'O', L. Sarto, N. Sanna and A. Martucci, "Comparison between Predicted and Actual Energy Performance for Summer Cooling in High-Performance Residential Buildings in The Lombardy Region (Italy)," Energy Build, vol. 54, pp. 234-242, 2012.

[193] A. Cormier, S. Robert, P. Roger, L. Stephan and E. Wurtz, "Towards a BIMbased Service Oriented Platform: Application to Building Energy Performance Simulation," in Proceedings of Building Simulation pp. 2309-2316, Sydney, 2011.

[194] R. Hitchcock and J. Wong, "Transforming IFC Architectural View BIMS For Energy Simulation," in Proceedings of Building Simulation 2011. pp. 1089-1095., Sydney, 2011.

[195] V. Bazjanac and A. Kiviniemi, "Reduction, Simplification, Translation and Interpretation In The Exchange of Model Data," in Proceedings of the 24th CIB W78 conference. pp. 163-168, Maribor, 2007.

[196] C. Wilkins and A. Kiviniemi, "Engineering-centric BIM," ASHRAE Journal, vol. 50, no. 12, pp. 44-48, 2008.

[197] A. Abdelalim, W. O’Brien and Z. Shi, "Visualization of Energy and Water Consumption and GHG Emissions: A Case Study of a Canadian University Campus," Energy and Buildings, vol. doi:10.1016/j.enbuild.2015.09.058, 2015. 
[198] D. Belzer, "Energy End-Use Flow Maps for the Buildings Sector," Pacific Northwest national Laboratory, Springfield, VA, 2006.

[199] phineas, "Visualizing Internal and External Heat Flow," Sankey Diagrams, 1 September 2015. [Online]. Available: http://www.sankeydiagrams.com/visualizing-internal-and-external-heat-flow/. [Accessed 10 December 2015].

[200] B. Dong, O. Zheng and Z. Li, "A BIM-enabled Information Infrastructure for Building Energy Fault Detection and Diagnostics," Automation in Construction, vol. 44, no. doi: http://dx.doi.org/10.1016/j.autcon.2014.04.007, pp. 197-211, 2014.

[201] D. Coakley, P. Raftery and P. Molloy, "Calibration of Whole Building Energy Simulation Models: Detailed Case Study of a Naturally Ventilated Building Using Hourly Measured Data," in First Building Simulation and Optimization Conference, no. September, 57-64, 2012.

[202] DOE, "Weather Data Sources," U.S. Department of Energy: Energy Efficiency and Renewable Energy, 2014. [Online]. Available:

http://apps1.eere.energy.gov/buildings/energyplus/weatherdata_sources.cfm\#TM Y2. [Accessed 26 October 2015].

[203] Simeb, "ExCalibBEM," Simeb, 2011. [Online]. Available: https://www.simeb.ca/ExCalibBEM/index_fr.php. [Accessed 3 October 2015].

[204] I. Beausoleil-Morrison, "The Adaptive Simulation of Convective Heat Transfer at Internal Building Surfaces," Building and Environment, vol. 37, no. 8-9 doi: http://dx.doi.org/10.1016/S0360-1323(02)00042-2, pp. 791-806, 2002.

[205] R. Perez, P. Ineichen and R. Seals, "Modelling Daylight Availability and Irradiance Components from Direct and Global Irradiance," Sol. Energy, vol. 44, no. 5 doi:10.1016/0038-092X(90)90055-H, p. 271-289, 1990.

[206] D. Thevenard and K. Haddad, "Ground Reflectivity in The Context of Building Energy Simulation," Energy and Buildings, vol. 38, no. 8 doi:

http://dx.doi.org/10.1016/j.enbuild.2005.11.007, pp. 972-980, 2006.

[207] Y. Pan, Z. Huang, G. Wu and C. Chen, "The Application Of Building Energy Simulation And Calibration In Two High-Rise Commercial Buildings In Shanghai," in Second National IBPSA-USA Conference, Cambridge, MA, 2006.

[208] I. MacDonald, "Quantifying The Effects of Uncertainty in Building Simulation," Ph.D. Thesis University of Strathclyde, United Kingdom, Strathclyde, United Kingdom, 2002. 
[209] F. S. Westphal and R. Lamberts, "Building Simulation Calibration using Sensitivity Analysis," in Ninth International IBPSA Conference, Montréal, Canada, 2005.

[210] Natural Resources Canada (NRCan), "HVAC \& Energy Systems," Natural Resources Canada, 05 March 2014. [Online]. Available: http://www.nrcan.gc.ca/energy/efficiency/housing/research/3937. [Accessed 2 November 2015].

[211] P. Herzog and L. LaVine, "Identification and Quantification of the Impact of Improper Operation of Midsize Minnesota Office Buildings on Energy Use: A Seven Building Case Study," in Proceedings of the ACEEE 1992 Summer Study on Energy Efficiency in Buildings (Volume 3), 1992.

[212] D. Claridge, J. Haberl, M. Liu, J. Houcek and A. Athar, "Can You Achieve 150\% Predicted Retrofit Savings? Is it Time for Re-Commissioning"," in Proceedings of the ACEEE 1994 Summer Study on Energy Efficiency in Buildings (Volume 5), American Council for and Energy Efficient Economy, 1994.

[213] T. I. Salsbury and C. D. Richard, "Performance Validation and Energy Analysis of HVAC Systems using Simulation," Energy \& Buildings, vol. 32, pp. 5-17, 2000 .

[214] A. S. 180-2008, "Standard Practice for Inspection and Maintenance of Commercial Building HVAC Systems," American Society of Heating, Refrigerating and Air-Conditioning Engineers, Atlanta, 2008.

[215] E. 15240:2007, "Ventilation for Buildings - Energy Performance of Buildings Guidelines for Inspection of Air-Conditioning Systems," EN 15240, 2007.

[216] Canada Green Building Council , "LEED Rating System for Existing Buildings: Operations \& Maintenance Rating System," LEED Canada, 2009.

[217] L. Pérez-Lombard , J. Ortiz , J. Coronel and I. Maestre, "A Review of HVAC Systems Requirements in Building Energy Regulations," Energy Build, vol. 43, no. 2, pp. 55-68, 2011.

[218] X. García-Casals , "Analysis of Building Energy Regulation and Certification in Europe: Their Role, Limitations and Differences," Energy Build, vol. 38, no. 5, pp. 81-92, 2006.

[219] L. Pérez-Lombard , J. Ortiz , R. González and I. Maestre , "A Review of Benchmarking, Rating and Labelling Concepts within The Framework of Building Energy Certification Schemes," Energy Build, vol. 41, no. 3, pp. 72-80, 2009. 
[220] L. Perez-Lombard, J. Ortiz and I. R. Maestre, "The Map of Energy Flow in HVAC Systems," Applied Energy, vol. 88, p. 5020-5031, 2011.

[221] P. Sakulpipatsin, L. Itard, H. van der Kooi, E. Boelman and P. Luscuere, "An Exergy Application for Analysis of Buildings and HVAC Systems," Energy and Buildings, vol. 42, pp. 90-99, 2010.

[222] B. Fan, X. Jin, X. Fang and Z. Du, "The Method of Evaluating Operation Performance of HVAC System Based on Exergy Analysis," Energy and Buildings, vol. 77, pp. 332-342, 2014.

[223] M. Ellen, J. Franconi and M. Brandemuehl, "Second Law study of HVAC distribution system performance," ASHRAE Transactions, vol. 105, p. 1246-2037, 1999.

[224] N. Mendes, R. M. Barbosa, R. Z. Freire and R. C. Oliveira, "A Simulation Environment for Performance Analysis of HVAC Systems," Build Simul, vol. 1, no. DOI 10.1007/s12273-008-8216-7, pp. 129-143, 2008.

[225] H. Friedman and M. A. Piette, "Comparative Guide to Emerging Diagnostic Tools for Large Commercial HVAC Systems," California Energy Commission (CEC), Report 48629 (http://eetd.lbl.gov/node/52146), 2001.

[226] N. Gayeski, S. Kleindienst, J. Gagne, B. Werntz, R. Cruz and S. Samouhos, "Data and Interfaces for Advanced Building Operations and Maintenance - RP 1633 Final Report," ASHRAE, Atlanta, 2015.

[227] S. Austin, "HVAC System Trend Analysis," ASHRAE Journal, pp. 44-50, 1997.

[228] A. Abdelalim, W. O'Brien and Z. Shi, "Data Visualization and Analysis of Energy Flow on a Multi-Zone Building Scale," Automation in Construction, p. Under review, 2016.

[229] phineas, "Sankey Diagrams," 13 November 2012. [Online]. Available: http://www.sankey-diagrams.com/tag/heat/page/2/. [Accessed 1 August 2016].

[230] Melbourne Energy Institute, The University of Melbourne, "Zero Carbon Australia: Buildings Plan," Beyond Zero Emissions , Fitzroy, Victoria, 2013.

[231] ASHRAE, "ASHRAE Handbook-HVAC Systems and Equipment," ASHRAE , 2012.

[232] Systemair, "Air Handling Units," Systemair, 2011.

[233] PRIVA, "Priva Top Control," Sustainable solutions for horticultural and building automation, 2011. [Online]. Available: http://www.priva.ca/en/products/priva-top- 
control. [Accessed 7 November 2015].

[234] E. Stamper and R. L. Koral, Handbook of Air Conditioning, Heating, and Ventilating, INDUSTRIAL PRESS INC, 1979.

[235] M. Liu, D. E. Claridge and S. Deng, "An Air Filter Pressure Loss Model for Fan Energy Calculation in Air Handling Units," International Journal of Energy Research, vol. 27, pp. 589-600, 2003.

[236] A. Bhatia, "HVAC - Guide to Demand Control Ventilation," ISBN-13: 9781502485656, 2014.

[237] A. T. Mustafa, "Experimental Study of Air Flow Rate Effects on Humidification Parameters with Preheating and Dehumidification Process Changing," ARPN Journal of Engineering and Applied Sciences, vol. 6, no. 9, pp. 63-68, 2011.

[238] J. Kao, "Sensor errors," ASHRAE, 1985.

[239] ASHRAE, "ASHRAE Handbook-Fundamentals (SI)," ASHRAE, https://www.ashrae.org/resources--publications/handbook/table-of-contents-2013ashrae-handbook--fundamentals, 2013.

[240] J. F. Straube, "Moisture in Buildings," ASHRAE Journal, pp. 15-19, 2002.

[241] EIA, U.S. Energy Information Administration, "Commercial Buildings Energy Consumption Survey (CBECS)," EIA, 17 May 2015. [Online]. Available: http://www.eia.gov/consumption/commercial/. [Accessed 27 October 2016].

[242] Natural Resources Canada (NRCan), "Energy Efficiency Trends in Canada, 1990 to 2009," National Resources of Canada, 09 July 2012. [Online]. Available: http://oee.rncan.gc.ca/publications/statistics/trends11/chapter3.cfm. [Accessed 27 October 2016].

[243] S. J. Hayter, P. A. Torcellini, R. B. Hayter and R. Judkoff, "The Energy Design Process for Designing and Constructing High-Performance Buildings," in Clima 2000/Napoli 2001 World Congress, Napoli, 2001.

[244] M. Asl, S. Zarrinmehr and W. Yan, "Towards BIM-based Parametric Building Energy Performance Optimization," in ACADIA 13: Adaptive Architecture [Proceedings of the 33rd Annual Conference of the Association for Computer Aided Design in Architecture ISBN 978-1-926724-22-5, Cambridge , 2013.

[245] W. Jeong, J. B. Kim, M. J. Clayton, J. S. Haberl and W. Yan, "Visualization of Building Energy Performance in Building Information Models," in ACADIA 13: Adaptive Architecture [Proceedings of the 33rd Annual Conference of the Association for Computer Aided Design in Architecture (ACADIA) ISBN 978-1- 
926724-22-5], Cambridge, 2013.

[246] M. Elnimeiri and M. Nicknam, "A Design Optimization Workflow for Tall Buildings using Parametric Algorithm," in Council on Tall Buildings and Urban Habitat, Seoul, 2011.

[247] Autodesk, "Better Building Performance," Autodesk, 2015. [Online]. Available: https://insight360.autodesk.com/oneenergy. [Accessed 27 October 2016].

[248] A. Abdelalim, Z. Shi and W. O'Brien, "Energy Flow Analysis on A Multi-Zonal Building Scale using Sankey Diagrams," in Proceedings of the eSim 2016 Building Performance Simulation Conference, Hamilton, Canada, 2016.

[249] A. Abdelalim, Z. Shi and W. O'Brien, "An Approach towards Developing Methods to Analyze and Visualize Energy Flow of HVAC System," in Society for Modeling \& Simulation International (SCS), London, UK, 2016.

[250] DOE, "Commercial Reference Buildings," U.S. Department of Energy, 2015. [Online]. Available: http://energy.gov/eere/buildings/commercial-referencebuildings. [Accessed 27 October 2016].

[251] Natural Resources Canada (NRCan), "Canada's energy code," Natural Resources Canada (NRCan), 19 January 2016. [Online]. Available:

http://www.nrcan.gc.ca/energy/efficiency/buildings/eenb/codes/4037. [Accessed 7 November 2016]. 


\section{Appendix A: Sankey Diagrams}

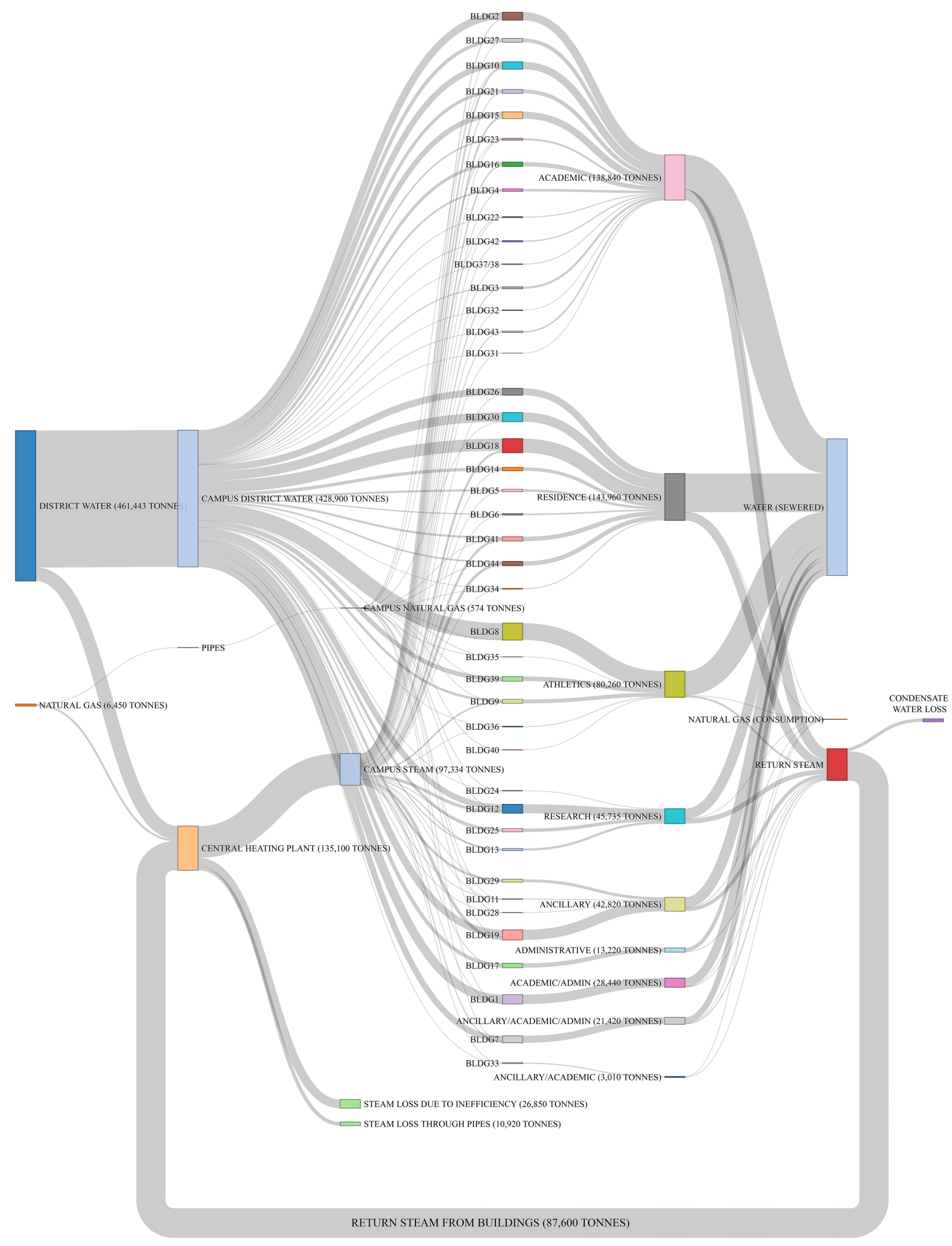

A. 1: Sankey diagram showing annual mass flow and its distribution by different sources 


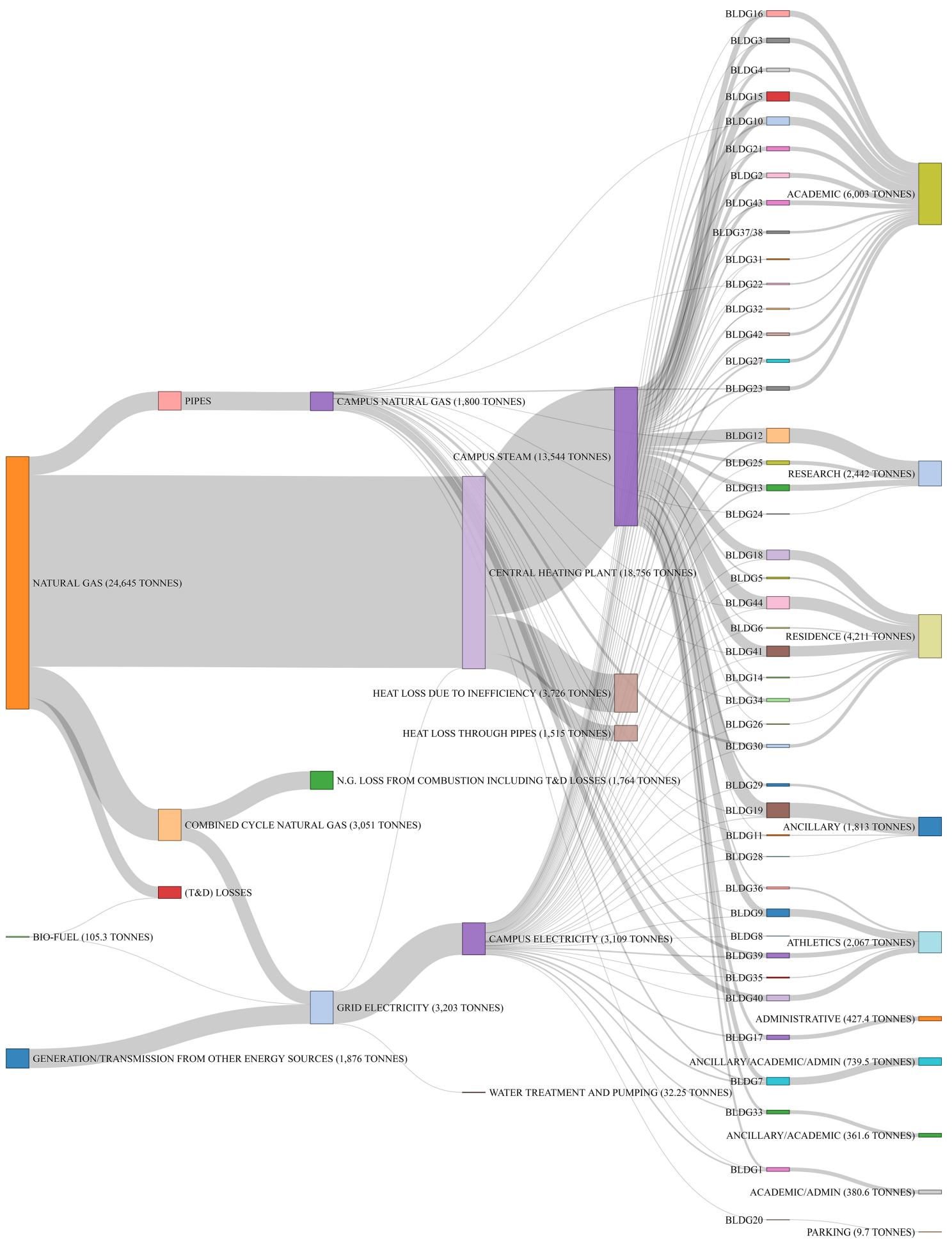

A. 2: Sankey diagram showing annual $\mathrm{CO} 2 \mathrm{e}$ emissions 


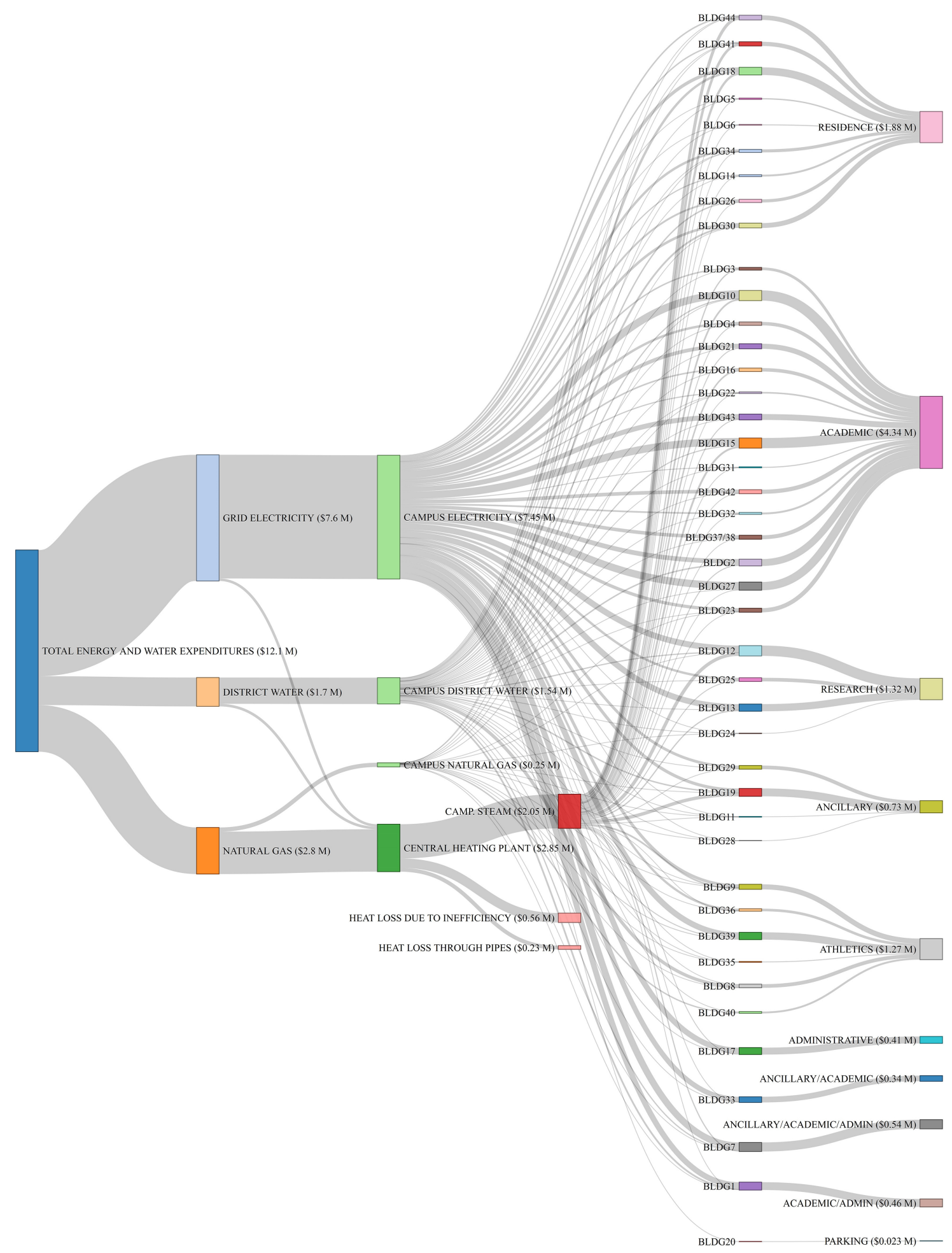

\section{A. 3: Sankey diagram showing annual utility cost}




\section{Appendix B: Normalized data per unit area and occupant}

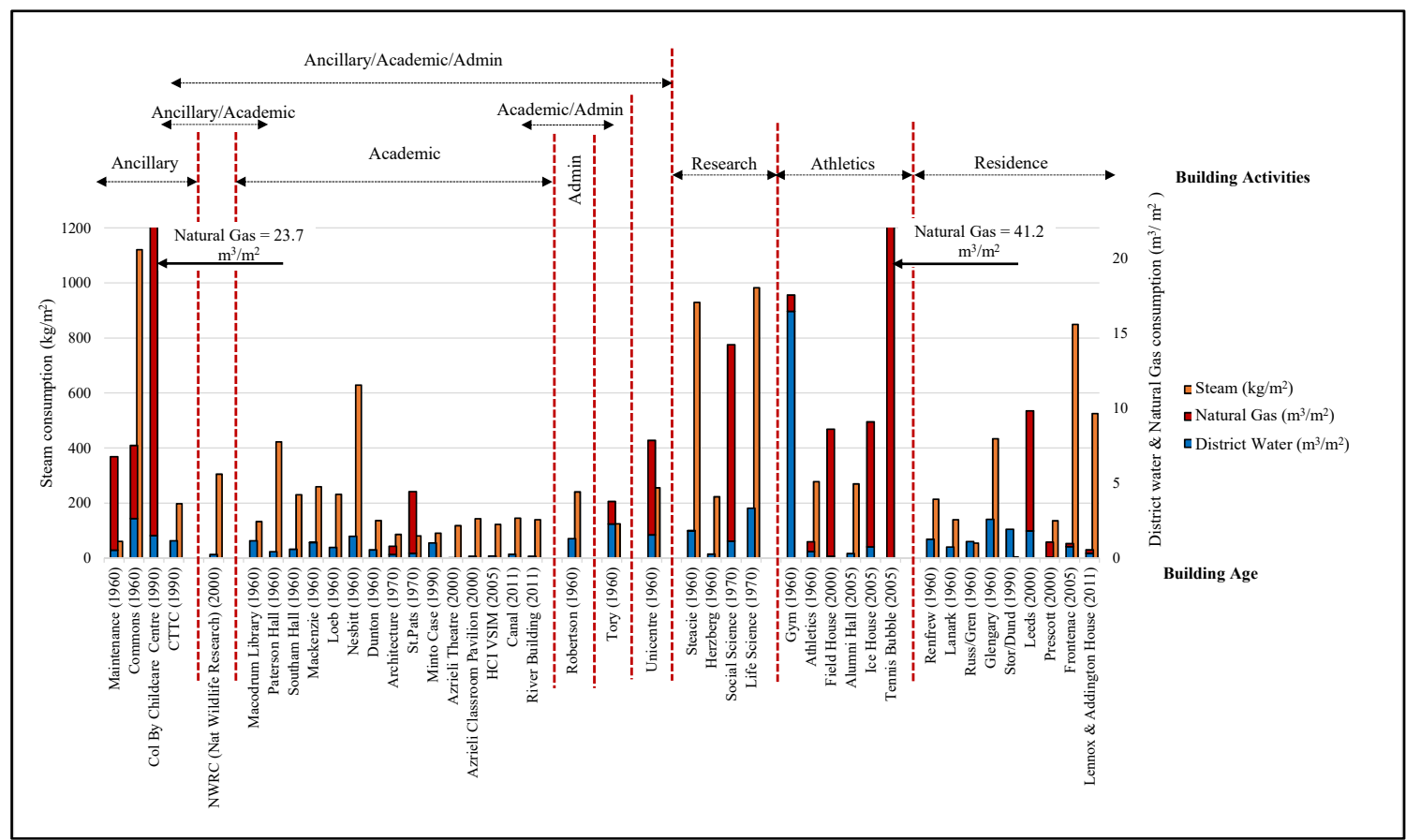

B. 1: Normalized district water, natural gas, and steam annual consumption per unit area

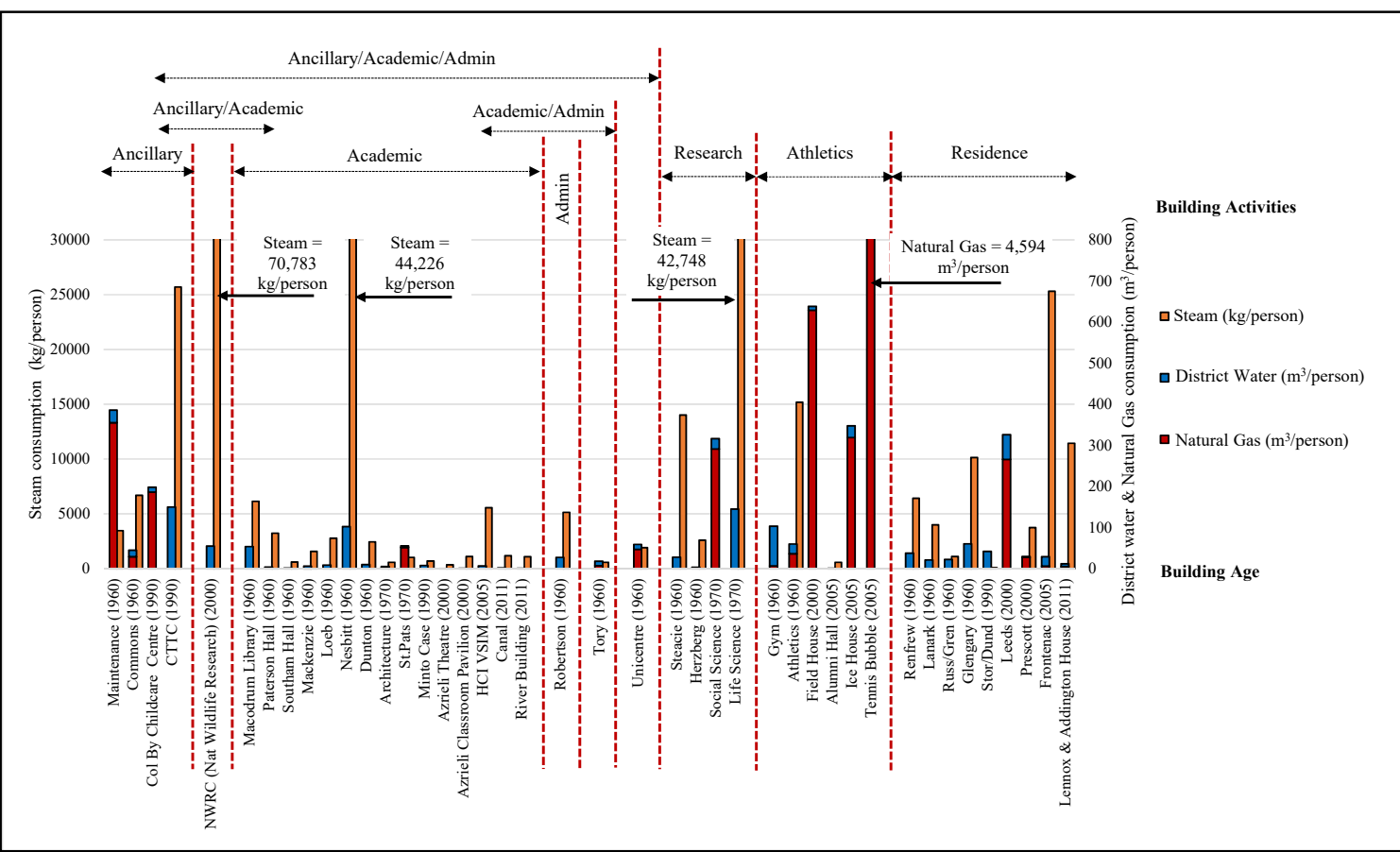

B. 2: Normalized district water, natural gas, and steam annual consumption per person 


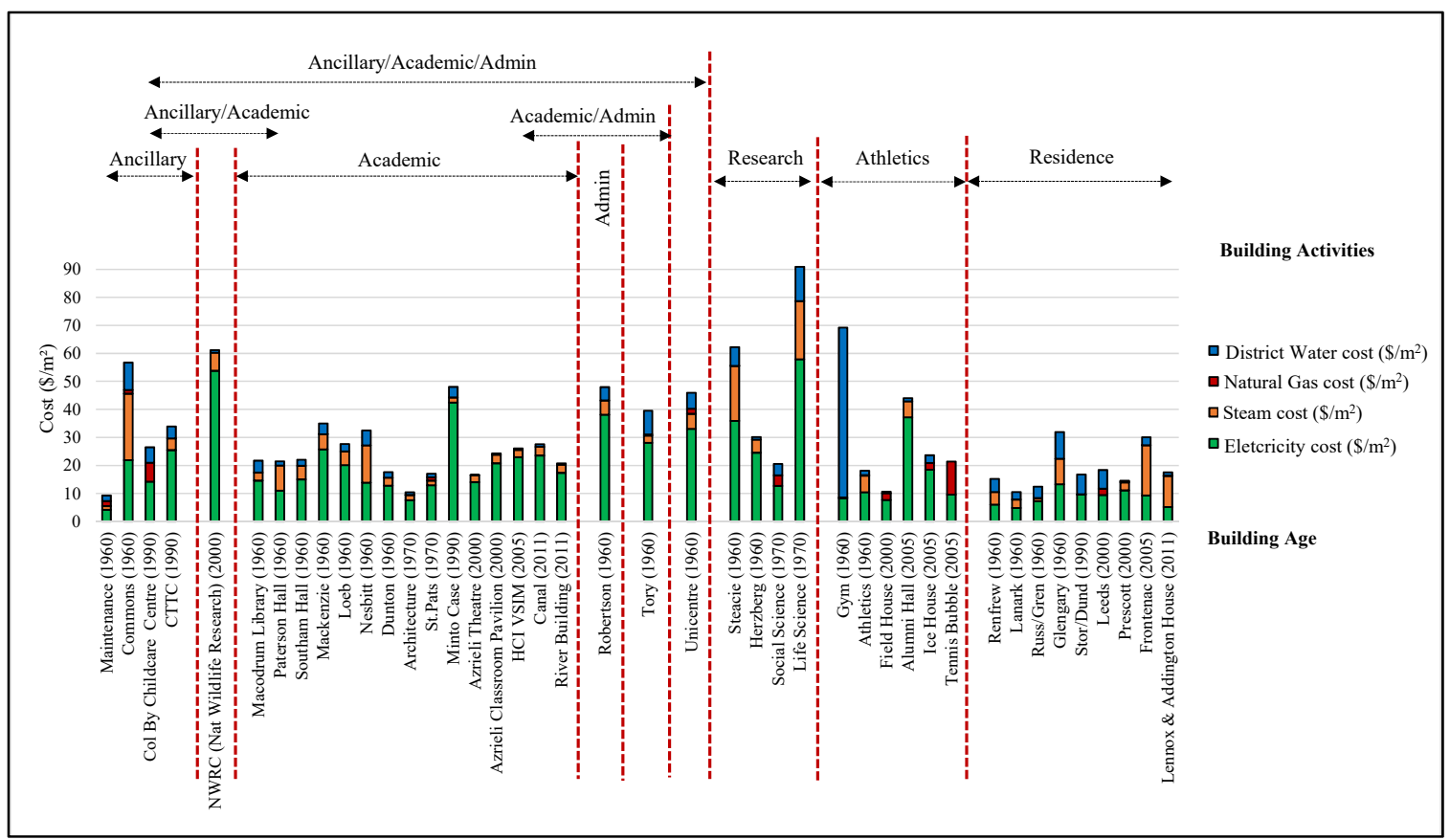

B. 3: Normalized annual utility cost per unit area

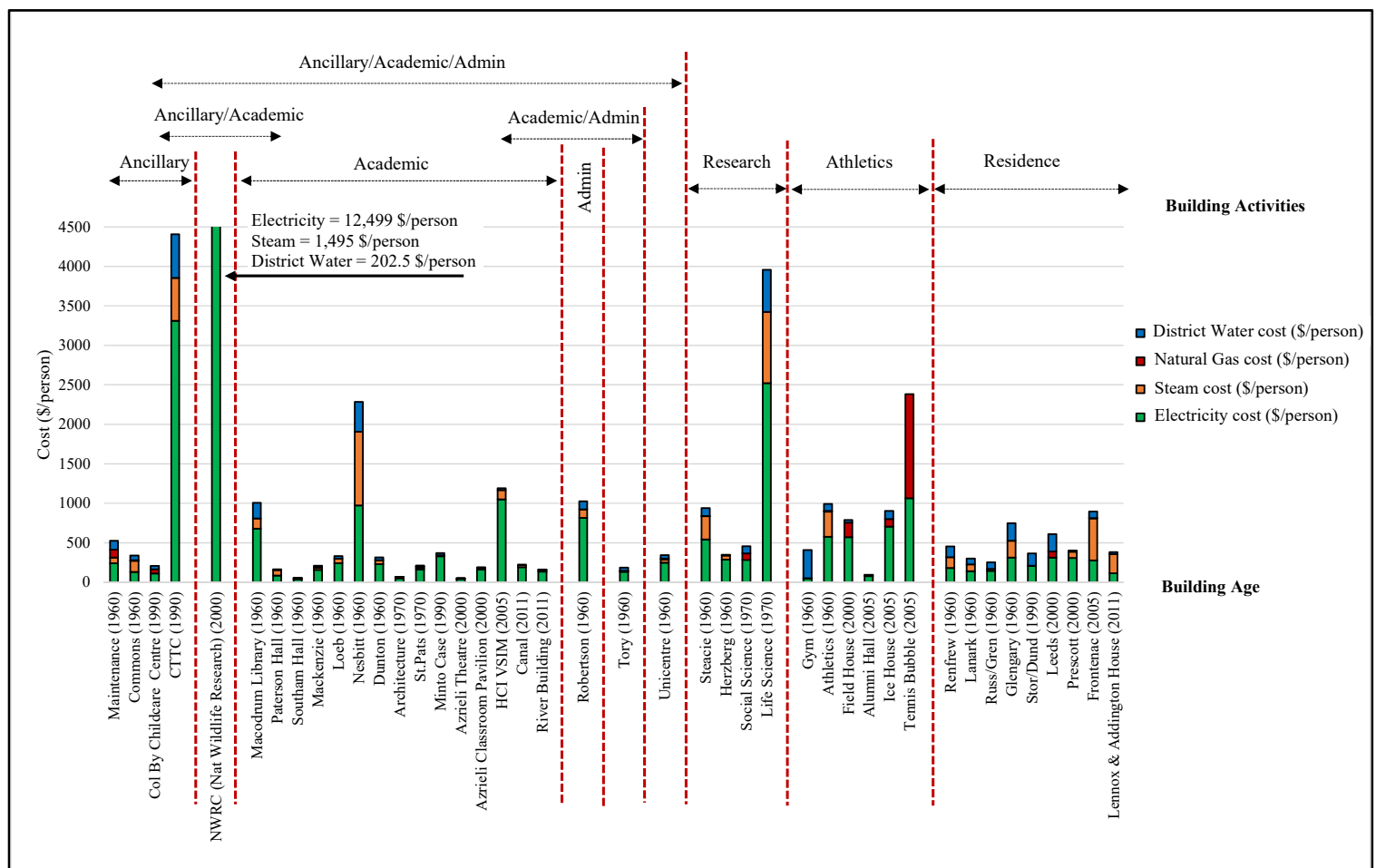

B. 4: Normalized annual utility cost per person 


\section{Appendix C: Survey Questions}

\section{1: Anonymous Survey on Evaluating Visualization Tools for Tracking Building/ Campus Energy and Water Use}

1) Is the current system that you regularly use for viewing building energy and water data effective for visualization? What are the weaknesses, if any, about current tools?

2) What types of data analysis do you perform?

Sankey diagrams: The following questions are related to Sankey diagrams. Samples of Sankey diagrams showing energy, water, and natural gas consumption, greenhouse gas emissions, and utility cost on annual basis for Carleton University were used in this survey. Samples of Sankey diagrams can be found in Section 3.5 and Appendix A. Note that line widths in Sankey diagrams are proportional to magnitude (e.g., energy or water use).

3) Are the above Sankey diagrams more effective for visualizing building performance over existing visualization methods/tools?

4) Are the above Sankey diagrams useful for facilitating decision making?

5) Do the above Sankey diagrams help in identifying abnormal behavior in energy and water consumption?

Bar graphs: The following questions are related to bar graphs. Below are samples of bar graphs showing normalized energy, water, and natural gas consumption, GHG emissions, and utility cost per unit area (square meter) on an annual basis. Samples of bar graphs can be found in Section 3.5 and Appendix B.

6) Are the above bar graphs more effective for visualizing building performance over 
existing visualization methods/tools?

7) Are the above bar graphs useful for facilitating decision making?

8) Do the above bar graphs help in identifying abnormal behavior in energy and water consumption?

\section{General questions:}

9) Is the data resolution (spatial and temporal) good enough in the Sankey diagrams and bar graphs for your purposes?

10) Which form of visualization (Sankey diagram or bar graphs) do you prefer? Why?

11) What other types of visualization or data would be useful for your job?

12) Do you have any other feedback? 


\section{2: Anonymous Survey on Evaluating the Effectiveness of Visualizing Energy} Flow and the Associated Cost Using Sankey Diagrams

This study has been applied to Canal Building at Carleton University in Ottawa, Canada. The building consists of seven floors with a total area of 8,000 sq. $\mathrm{m}$ and includes a large variety of functional space.

1) Which of the following best describes your profession?
a. Energy manager
b. Mechanic
c. Design engineer
d. Technician
e. Other building-related technician

2) How many years of experience do you have with a building energy performancerelated profession?
a. $0-5$
b. $5-10$
c. $10-20$
d. More than 20

General Questions: The following questions are related to visualizing energy flow on the building level using Sankey diagrams. Samples of Sankey diagram showing energy flows during heating and cooling seasons were used in this study. Sankey diagrams visualizing energy flows can be found in Section 4.5. Note that line widths in Sankey diagrams are proportional to magnitude. Energy enters the building from the left and leaves to the right.

3) These Sankey diagrams are effective visualizing building-level energy flows.
a. Strongly agree
b. Agree
c. Neural
d. Disagree
e. Strongly disagree 
4) These Sankey diagrams help you understand the interrelated variables that affect building performance (such as the effect of internal gains to the HEATING and COOLING loads).
a. Strongly agree
b. Agree
c. Neural
d. Disagree
e. Strongly disagree

5) These Sankey diagrams help you identify system inefficiencies.
a. Strongly agree
b. Agree
c. Neural
d. Disagree
e. Strongly disagree

6) These Sankey diagrams help you understand unmeasured energy flows (e.g. solar gains, infiltration and ventilation).
a. Strongly agree
b. Agree
c. Neural
d. Disagree
e. Strongly disagree

7) These Sankey diagram have resolution (spatial and temporal) that is sufficient to understand the building's energy performance.
a. Strongly agree
b. Agree
c. Neural
d. Disagree
e. Strongly disagree

Interpreting Energy Flows: The same Sankey diagrams shown in the previous section were used.

8) From the COOLING season Sankey diagram that shows energy flows, which path is responsible for the most COOLING energy? 

a. Windows
b. Opaque envelope components
c. Infiltration
d. Natural ventilation
e. People
f. Lighting
g. Equipment/appliances
h. I don't understand

9) From the COOLING season Sankey diagram that shows energy flows, what is the most significant path for heat removal from the building?
a. Windows
b. Opaque envelope components
c. Infiltration
d. Natural ventilation
e. I don't understand

10) From the HEATING season Sankey diagram that shows energy flows, which path is responsible for the most HEATING energy?
a. Windows
b. Opaque envelope components
c. Infiltration
d. Natural ventilation
e. Exhaust air
f. I don't understand

11) From the HEATING season Sankey diagram that shows energy flows, which heat source has the biggest impact on reducing steam consumption?
a. Windows
b. People
c. Lighting
d. Equipment/appliances
e. I don't understand

Energy Cost: The following questions are related to visualizing energy cost on the building level using Sankey diagrams. The purchased energy (steam and electricity) are directly translated to costs, while the indirect energy flows are also attributed to indirect 
costs. Sankey diagram showing energy cost during heating and cooling seasons were used in this section. Sankey diagrams visualizing energy cost flows can be found in Section 4.5.

12) Are the Sankey diagrams effective in visualizing energy cost on the building level? Why or why not?

13) What is the ratio of passive to active energy cost during HEATING seasons?
a. $0-10 \%$
b. $10-20 \%$
c. $20-30 \%$
d. $30-40 \%$
e. More than $50 \%$

14) What is the ratio of passive to active energy cost during COOLING seasons?
a. $0-10 \%$
b. $10-20 \%$
c. $20-30 \%$
d. $30-40 \%$
e. More than $50 \%$

15) Which single building component would be most effective at reducing HEATING and COOLING cost?
a. Windows
b. Opaque envelope components
c. Infiltration
d. Natural ventilation
e. Lighting
f. Equipment/appliances

16) Does your above decision lead to a reduction or an increase in HEATING and COOLING cost? and why?

\section{General Questions}

17) Overall, Sankey diagrams useful for facilitating decision making.
a. Very likely 

b. Somewhat likely
c. Not sure
d. Not very likely
e. Very unlikely

18) What tools, if any, do you currently use for visualizing building performance?

19) If Sankey diagrams like the ones shown above were available to you for your building(s), how likely would you be to use them?
a. Very likely
b. Somewhat likely
c. Not sure
d. Not very likely
e. Very unlikely

20) How could the Sankey diagrams be improved to be more useful to visualize energy flows and the associated cost on the building level? 


\section{Appendix D: Hypertext Markup Language (HTML) Code}

26

\# The HTML code developed helped in organizing the structure of the web page including controls (such as number of diagrams, upload files, colors, and fonts). \# This work is completed through a research contract funded by the Natural Resources Canada in 2017.

\# The script presented here is for information only. Researchers assume no liability for any inaccurate, delayed or incomplete information, nor for any actions taken in reliance thereon.

$<$ br clear="all" $><$ !DOCTYPE html $>$

$<$ meta charset="utf-8">

$<$ title>SANKEY Experiment</title>

$<$--- Latest compiled and minified CSS -->

$<$ link rel="stylesheet" href="resources/bootstrap.min.css">

$<!--$ Latest compiled JavaScript -->

<script src="https://ajax.aspnetcdn.com/ajax/jQuery/jquery-3.1.1.min.js"></script>

$<$ !-- Latest compiled and minified CSS -->

$<$ link

href="https://maxcdn.bootstrapcdn.com/bootstrap/3.3.7/css/bootstrap.min.css">

rel="stylesheet"

$<!--$ Latest compiled JavaScript -->

<script src="https://maxcdn.bootstrapcdn.com/bootstrap/3.3.7/js/bootstrap.min.js"></script>

<script src="https://code.jquery.com/jquery-1.12.4.js"></script>

<script src="https://code.jquery.com/ui/1.12.1/jquery-ui.js"></script>

<link rel="stylesheet" href="http://code.jquery.com/ui/1.9.2/themes/base/jquery-ui.css">

<style>

.nav-tabs $\{$ border-bottom: $2 p x$ solid \#DDD; $\}$

.nav-tabs $>$ li.active $>$ a, .nav-tabs $>$ li.active $>$ a:focus, .nav-tabs $>$ li.active $>$ a:hover $\{$ borderwidth: 0 ; \}

.nav-tabs > li > a \{ border: none; color: \#666; $\}$

.nav-tabs > li.active > a, .nav-tabs > li > a:hover \{ border: none; color: \#4285F4 !important; background: transparent; $\}$

.nav-tabs > li > a::after \{ content: "'; background: \#4285F4; height: 2px; position: absolute; width:

$100 \%$; left: 0px; bottom: $-1 \mathrm{px}$; transition: all $250 \mathrm{~ms}$ ease 0 s; transform: scale(0); \}

.nav-tabs > li.active > a::after, .nav-tabs > li:hover > a::after $\{$ transform: scale(1); $\}$

.tab-nav > li > a::after \{ background: \#21527d none repeat scroll 0\% 0\%; color: \#fff; \}

tab-pane \{ padding: $15 \mathrm{px} 0 ;\}$

.tab-content\{padding:20px\}

.card \{background: \#FFF none repeat scroll 0\% 0\%; box-shadow: 0px 1px 3px rgba(0, 0, 0, 0.3); margin-bottom: 30px; $\}$

body\{ background: \#EDECEC; padding:50px\}

$</$ style $>$

$<$ style>

.overlay \{

background: \#e9e9e9;

display: block;

position: absolute;

right: 0;

left: 0;

opacity: 0.7 ;

z-index: 20; 


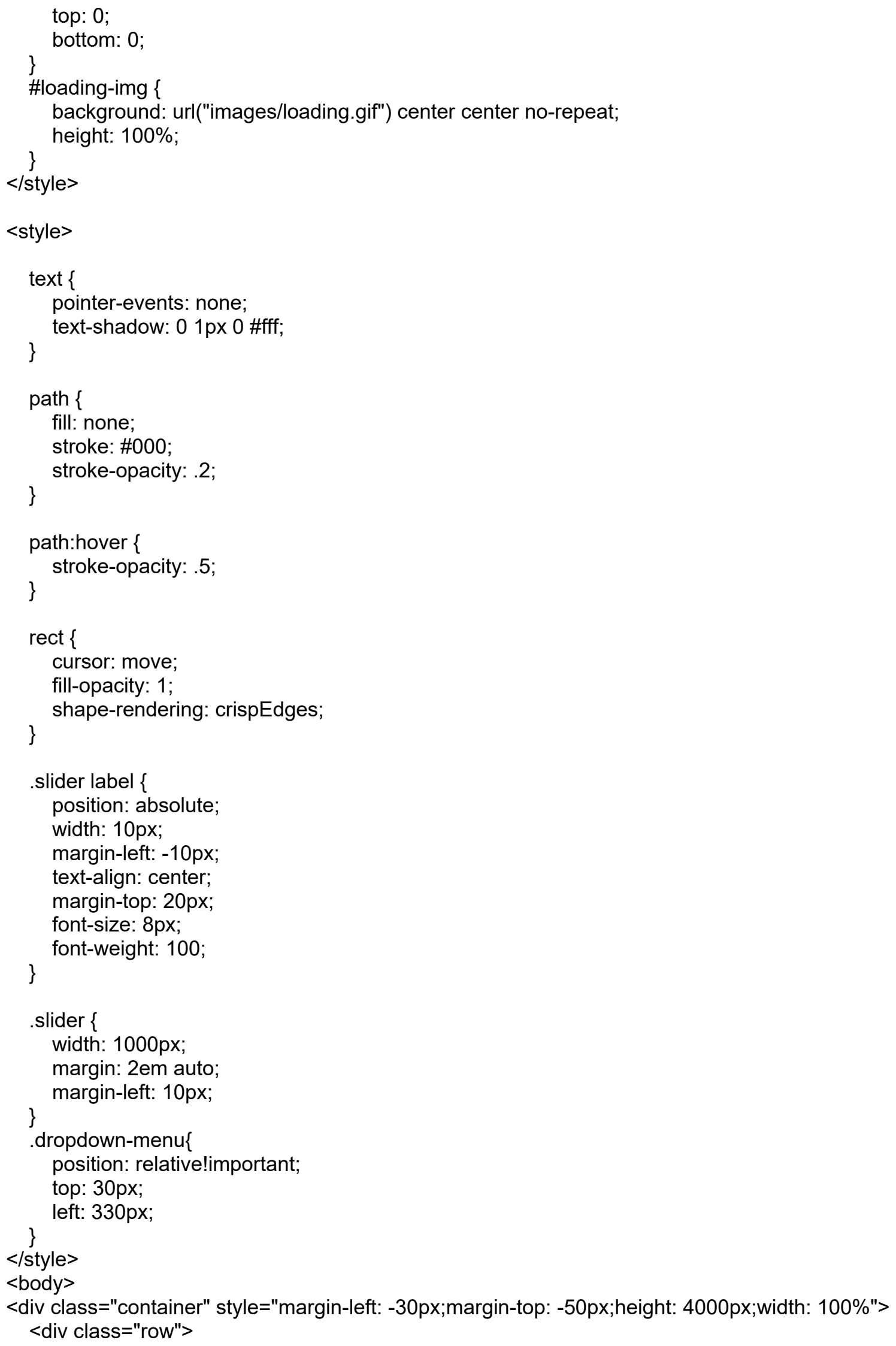




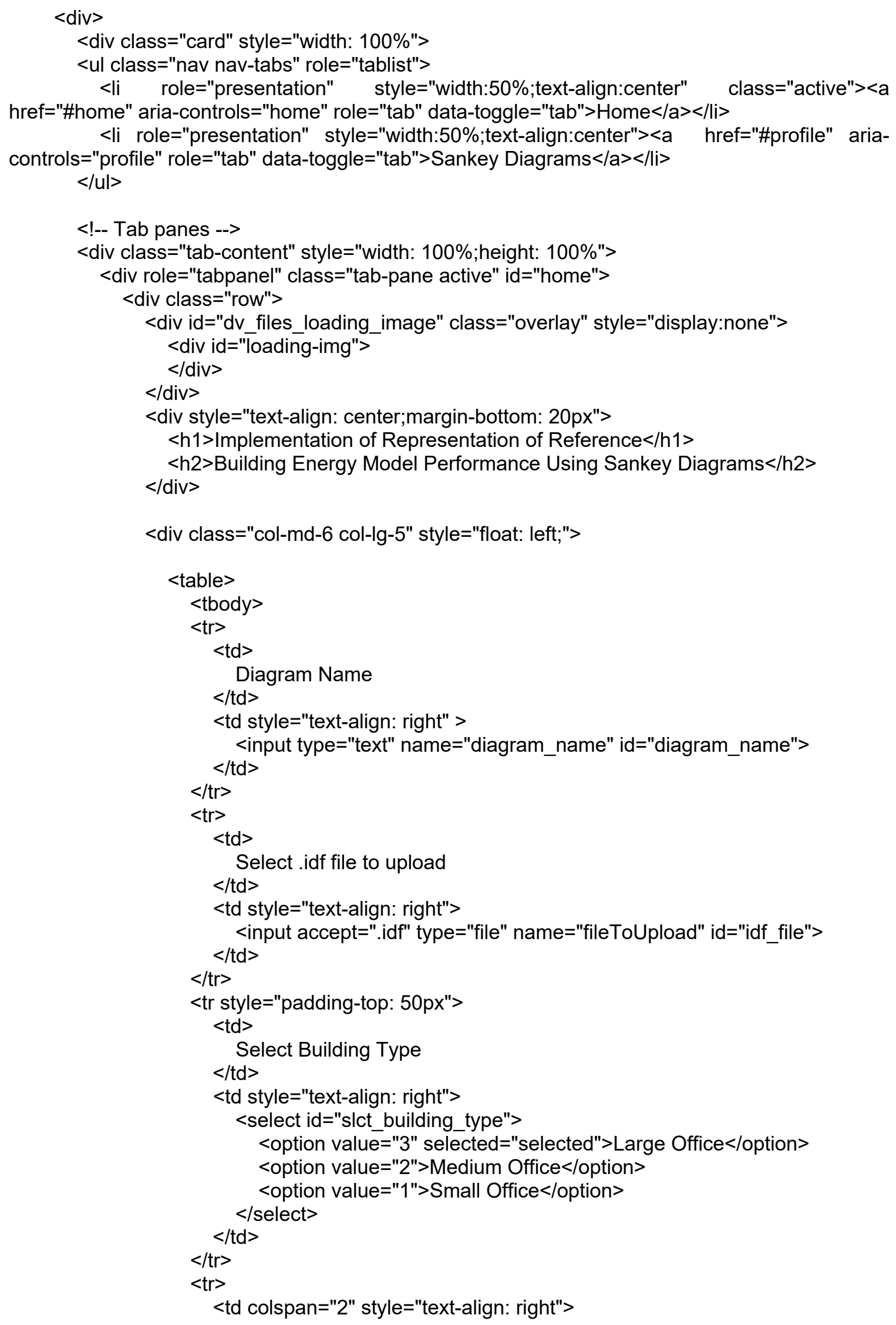




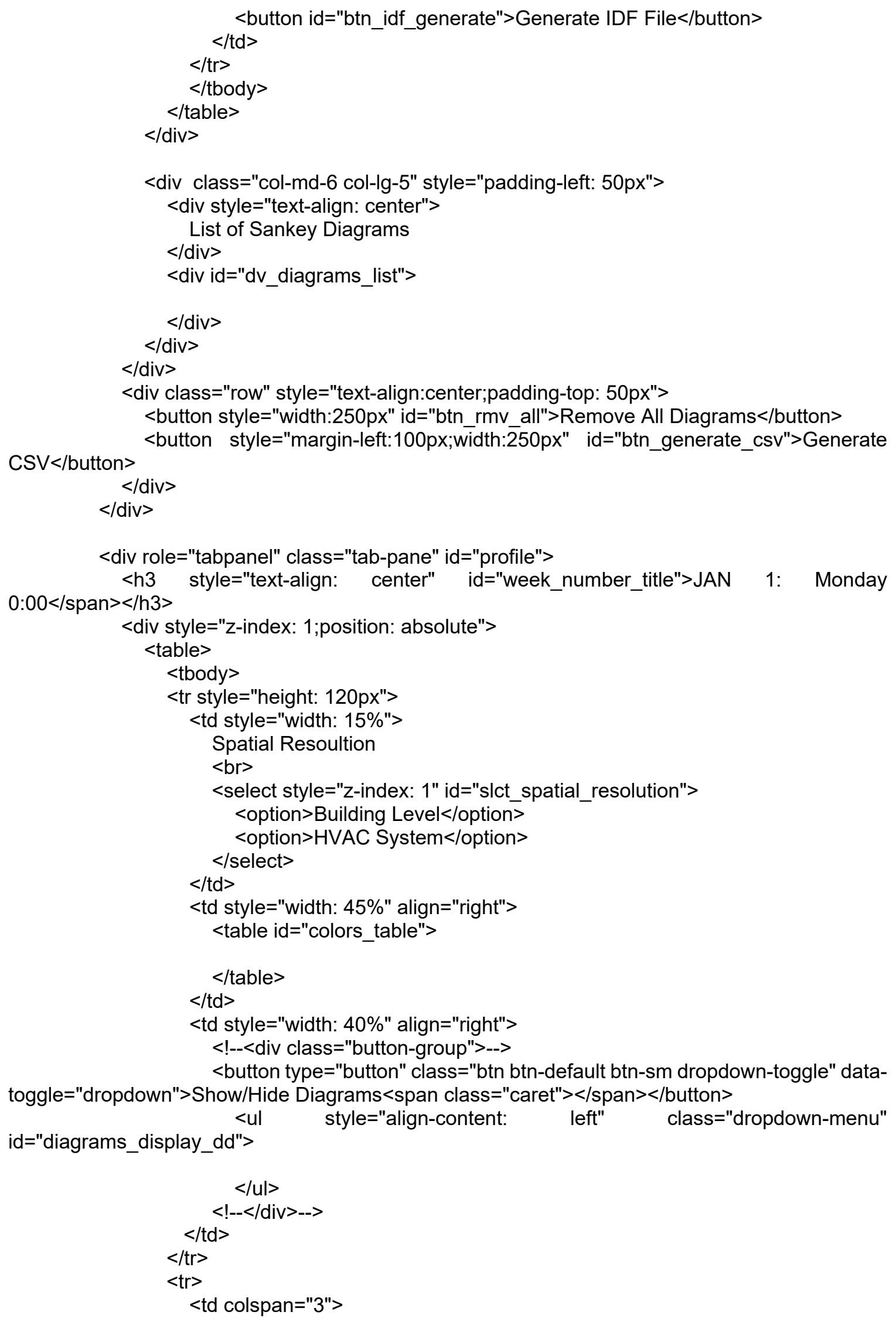


Slideshow

<button id="btn_slideshow" onclick="ToggleSlideShow()" style="font-size: $14 p x "><$ span id="span_slideshow" class="glyphicon glyphicon-play" $></$ span $></$ button $>$ style="font-size: $14 p x ">1.0 x</$ button $>$ id="btn_slideshow_speed" onclick="SpeedSlideShow()" $</ \mathrm{td}>$

$</$ tr $>$

$<\operatorname{tr}>$

$<\mathrm{td}>$

<input type="radio" name="slider_radio" checked="checked"

value="hours">Hours [Unit: W]

$</ \mathrm{td}>$

$<$ td colspan="2">

$<$ div class="slider" id="hours_slider" $></$ div $>$ $</ \mathrm{td}>$

$</$ tr $>$

$<\operatorname{tr}>$

$<\mathrm{td}>$

$<$ input type="radio" name="slider_radio" value="days">Days [Unit: kWh] $</$ td $>$

$<$ td colspan="2">

$<$ div class="slider" id="days_slider" $></$ div $>$ $</$ td $>$

$<$ tr $>$

$<$ tr>

$<\mathrm{td}>$

$\mathrm{kWh}]$

<input type="radio" name="slider_radio" value="months" $>$ Months [Unit:

$</ \mathrm{td}>$

$<$ td colspan="2">

$<$ div class="slider" id="months_slider" $></$ div $>$ $</$ td $>$

$</$ tr $>$

$</$ tbody $>$

$<$ table $>$

$</$ div $>$

$<$ div id="chart" $></$ div $>$

$</$ div $>$

$</$ div $>$

$</$ div $>$

$</$ div $>$

$</$ div $>$

$<$ div $>$

<script type="text/javascript" src="resources//d3.v3.min.js"></script $>$

$<$ script type="text/javascript" src="resources/sankey.js" $></$ script $>$

<script type="text/javascript" src="resources/global_vars.js" $></$ script $>$

$<$ script type="text/javascript" src="resources/spatial_resolution.js" $></$ script $>$

<script type="text/javascript" src="resources/slideshow.js" $></$ script $>$

$<$ script type="text/javascript" src="resources/colors_selector.js" $></$ script $>$

$<$ script type="text/javascript" src="resources/diagrams_dropdown.js" $></$ script $>$

$<$ script type="text/javascript" src="resources/sliders.js" $></$ script $>$ 
278 <script type="text/javascript" src="resources/generic_sankey.js" $></$ script $>$

279 <script type="text/javascript" src="resources/main_page.js" $></$ script $>$

280 <script type="text/javascript" src="resources/document_ready.js" $></$ script $>$

$281</$ body $>$

$282</$ html $>$ 


\section{Appendix E: JavaScript Codes}

\section{Appendix E1: JavaScript code providing functions and controls for the "HOME"}

\section{tab}

1

\# This JavaScript code aims to provide functions and controls for the "HOME" tab of the web page. \# This work is completed through a research contract funded by the Natural Resources Canada in 2017.

\# The script presented here is for information only. Researchers assume no liability for any inaccurate, delayed or incomplete information, nor for any actions taken in reliance thereon.

var current_id = 1;

\$("\#btn_idf_generate").click(function () \{

var diagram_name $=\$($ "\#diagram_name").val();

var building_type_id = \$( "\#slct_building_type option:selected" ).val();

var building_type_name $=\$($ "\#slct_building_type option:selected" ).text();

var files = \$('\#idf_file').prop("files");

var formData $=$ new FormData();

formData.append('idf_file', files[0]);

formData.append('building_type_id', building_type_id);

formData.append('diagram_name', diagram_name);

\$.ajax $(\{$

url: "index.php/idf/generate/",

data: formData,

enctype: 'multipart/form-data',

processData: false,

contentType: false,

cache:false,

type: 'POST',

success: function(response) \{

if(response.success)

\{

var file_name = response.generated_idf_filename;

document.location = "index.php/idf/download/" + file_name;

var new_div = '<div style="text-align: left" >' +

"Diagram Name:" + diagram_name + "<br $>$ " +

"Building Type:"+building_type_name+"<br>" +

'Upload ESO file <input class="js-upload-eso" data-diagram="'+diagram_name+"' accept=".eso" type="file" name="fileToUpload" id="idf_file">'

+ '<button data-diagram="'+diagram_name+"' class="js-remove-diagram" $>$ Remove

Diagram $</$ button $>$ '

+ '</div>';

$\$\left(" \# d v \_d i a g r a m s \_l i s t "\right) . a p p e n d\left(n e w \_d i v\right) ;$

BindRemoveEvent();

\$(".js-upload-eso").on("change", function() \{ event.preventDefault();

var form = new FormData();

files $=\$$ (this).prop("files");

form.append(\$(this).data("diagram"), files[0]);

console.log(files);

\$("\#dv_files_loading_image").show();

\$.ajax $\overline{(\{}$ 


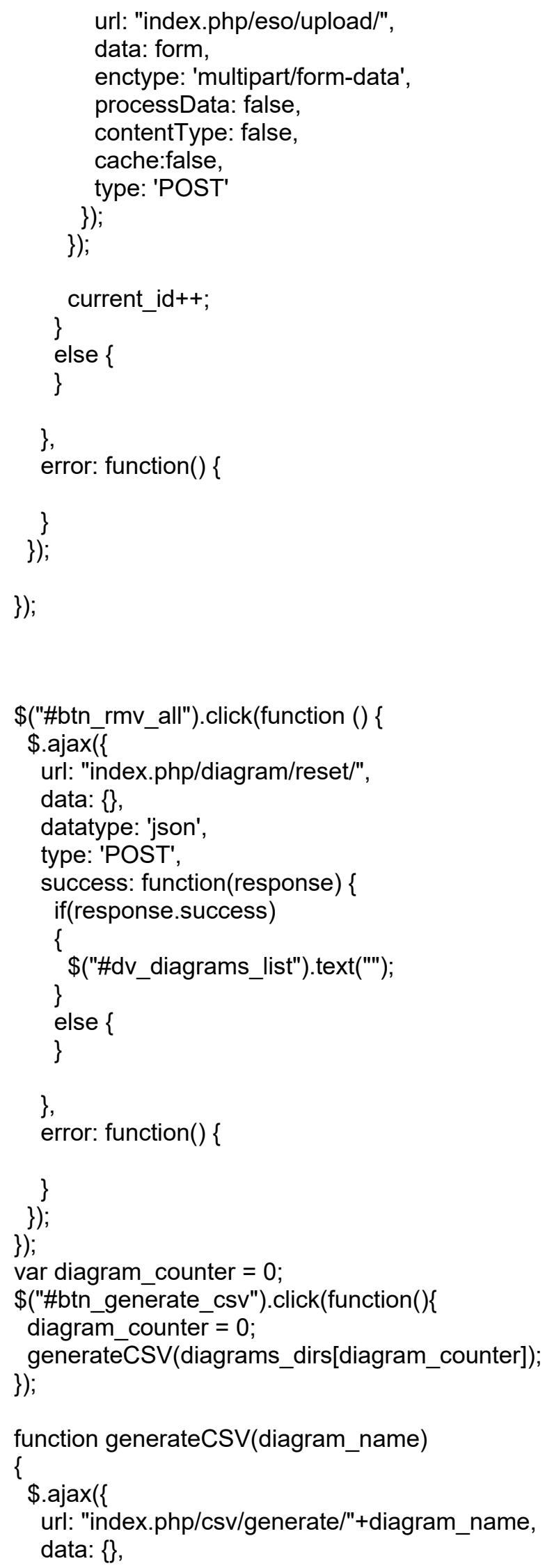




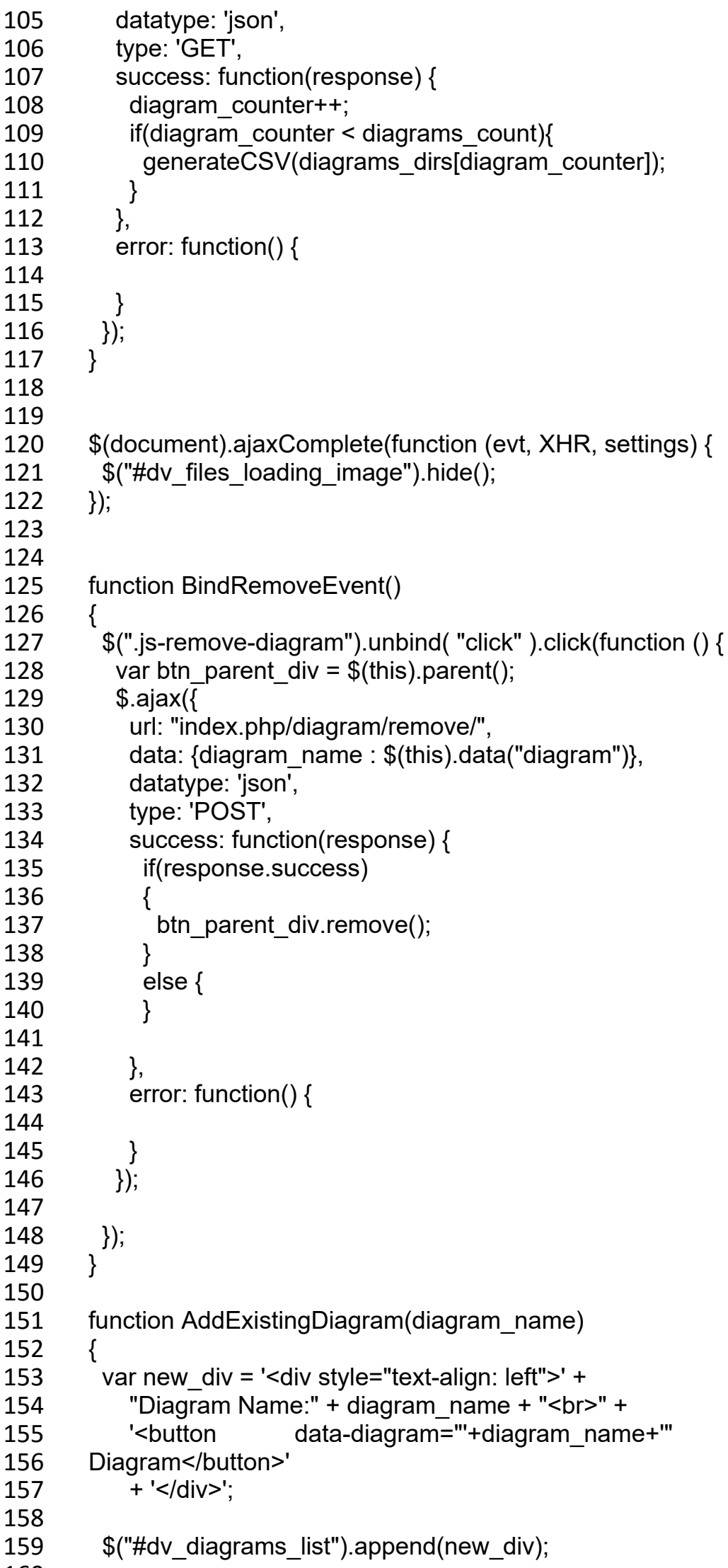


161 BindRemoveEvent();

$162\}$ 


\section{Appendix E2: D3's Sankey layout JavaScript code}

\# This JavaScript code shows D3's Sankey layout used to construct Sankey diagrams. \# This work is completed through a research contract funded by the Natural Resources Canada in 2017.

\# The script presented here is for information only. Researchers assume no liability for any inaccurate, delayed or incomplete information, nor for any actions taken in reliance thereon.

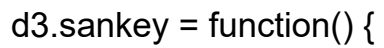




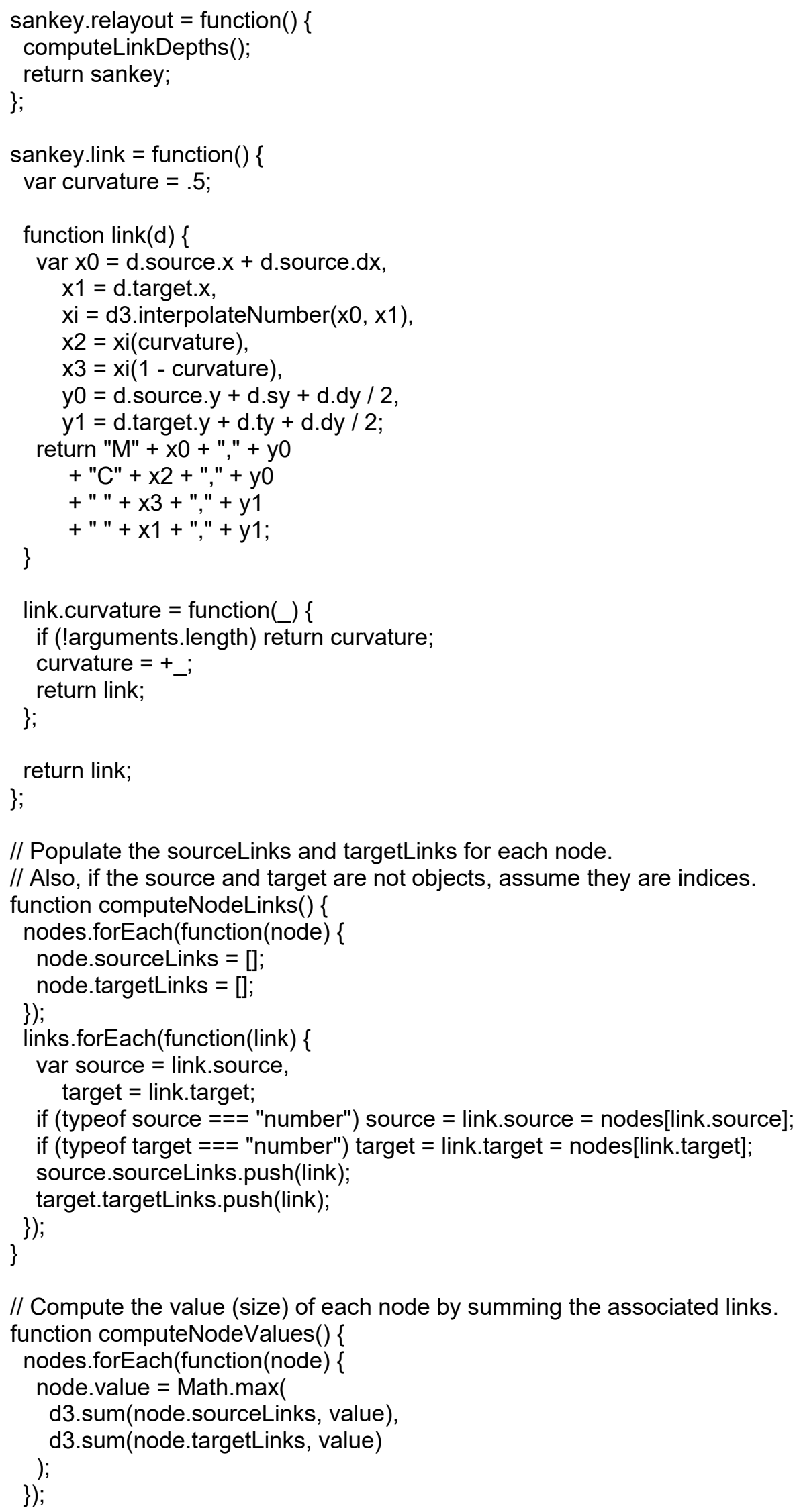




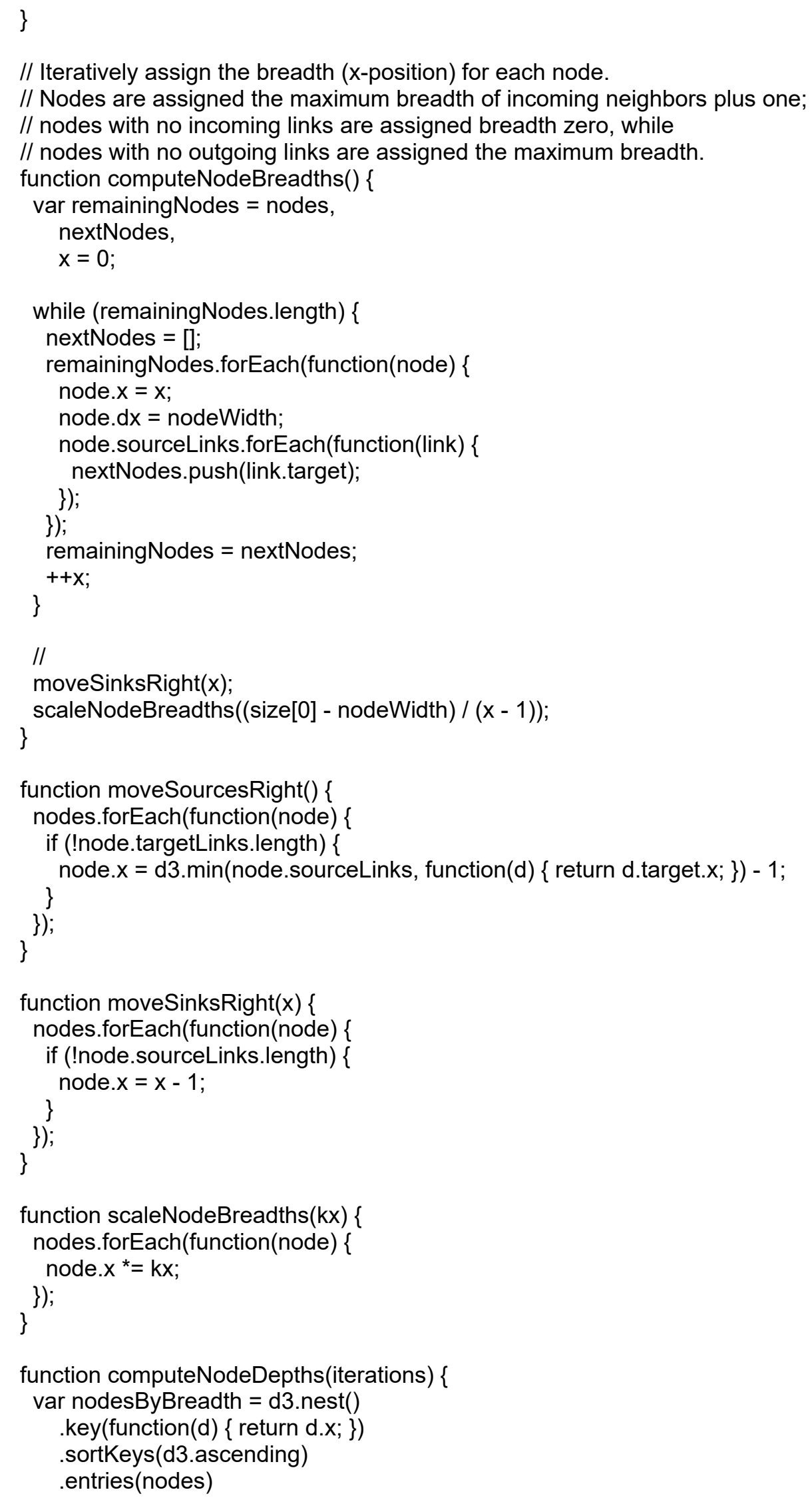




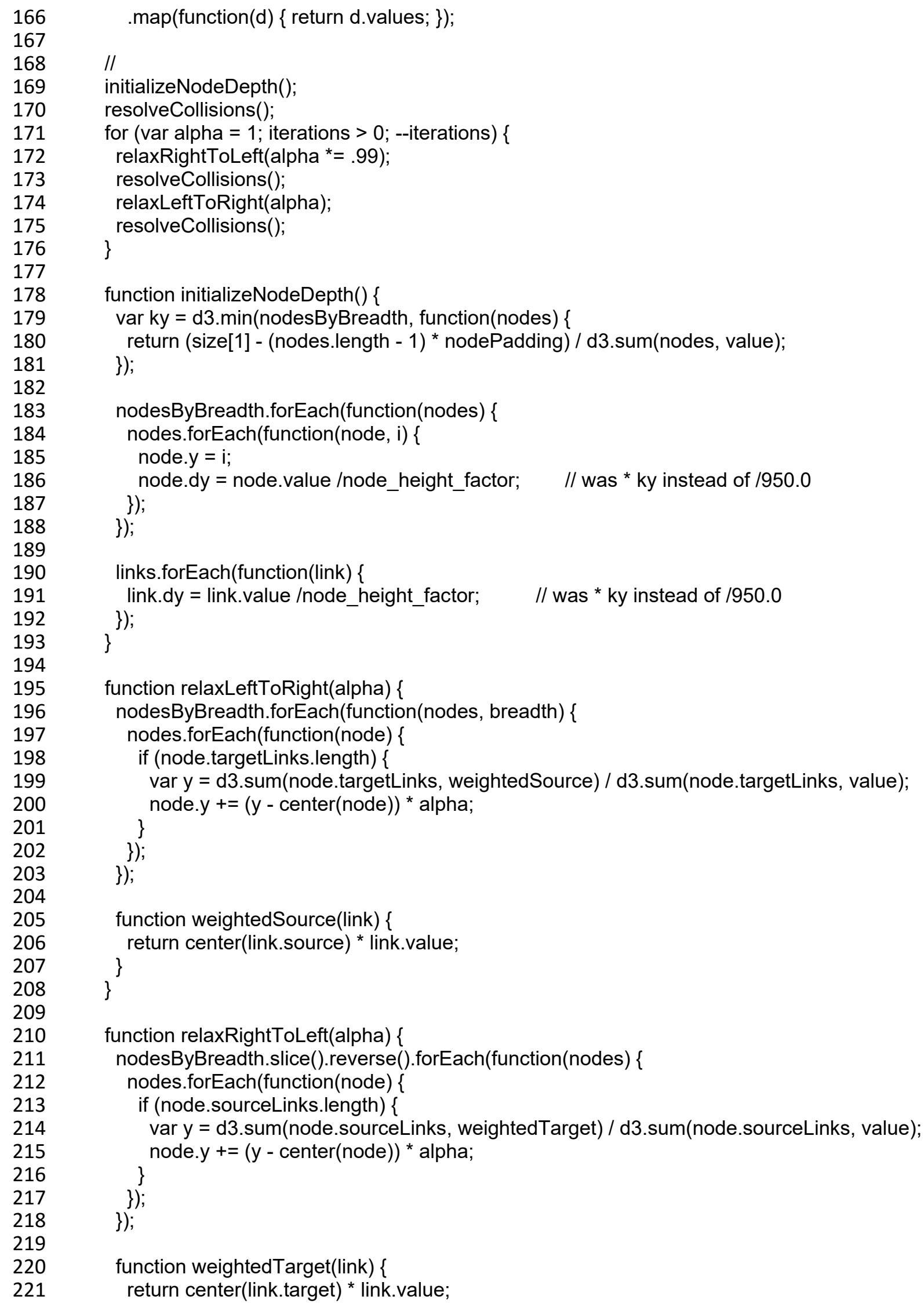




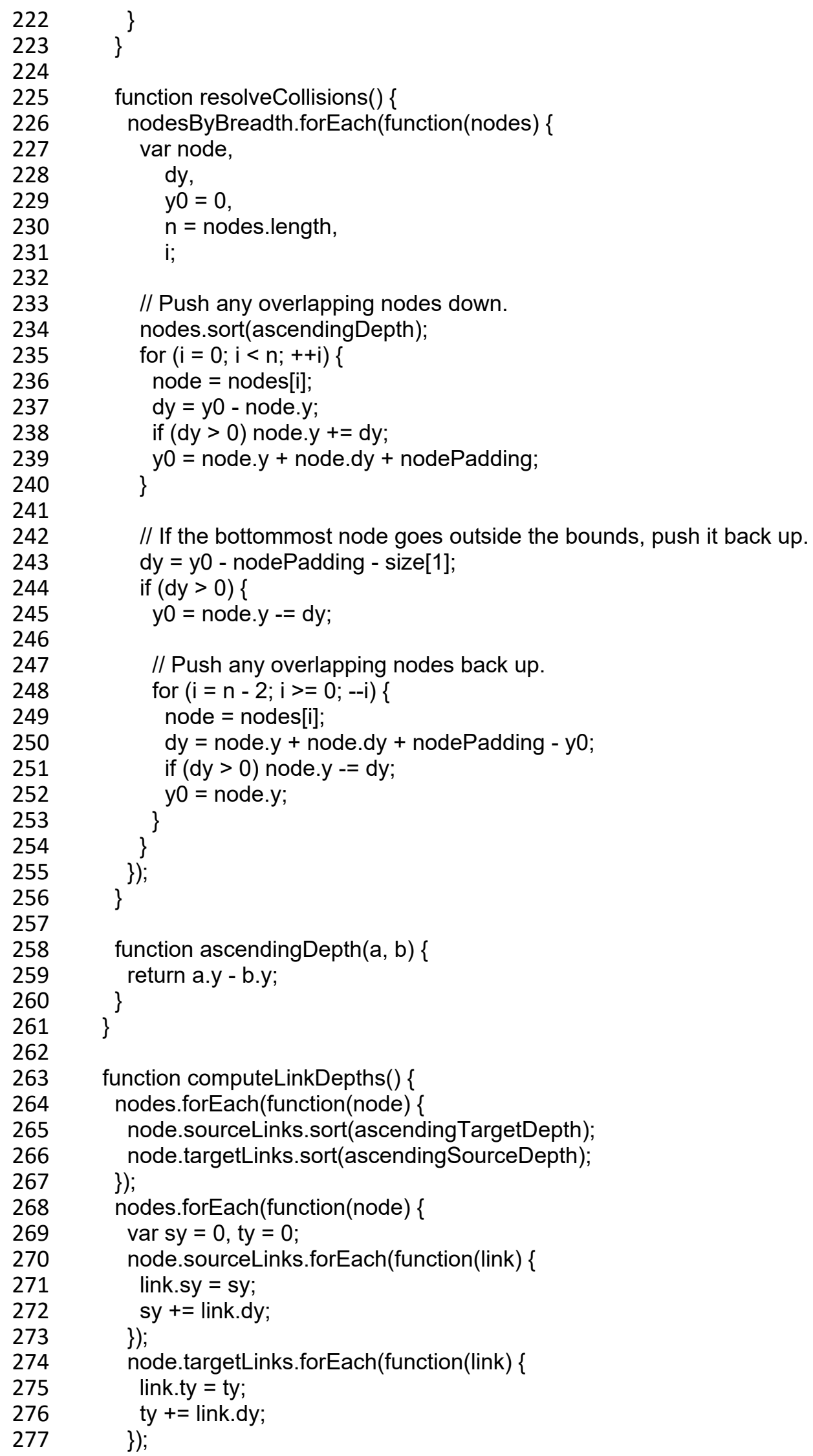




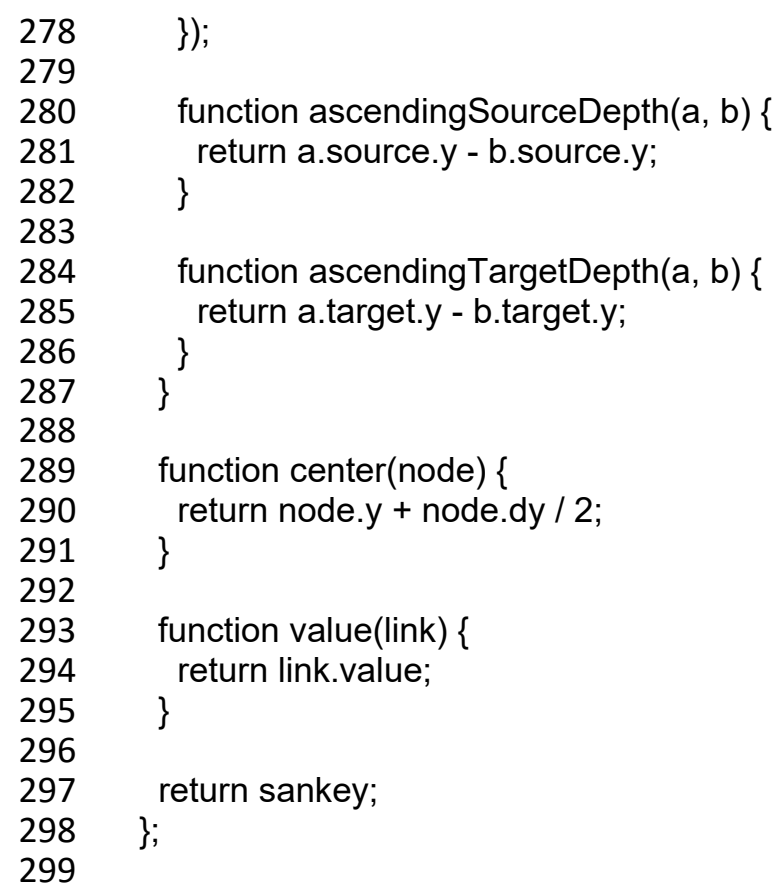




\section{Appendix E3: List of variables}

1 \# This JavaScript code lists the variables that are used by other JavaScript codes.

2 \# This work is completed through a research contract funded by the Natural Resources Canada in 32017.

4 \# The script presented here is for information only. Researchers assume no liability for any 5 inaccurate, delayed or incomplete information, nor for any actions taken in reliance thereon.

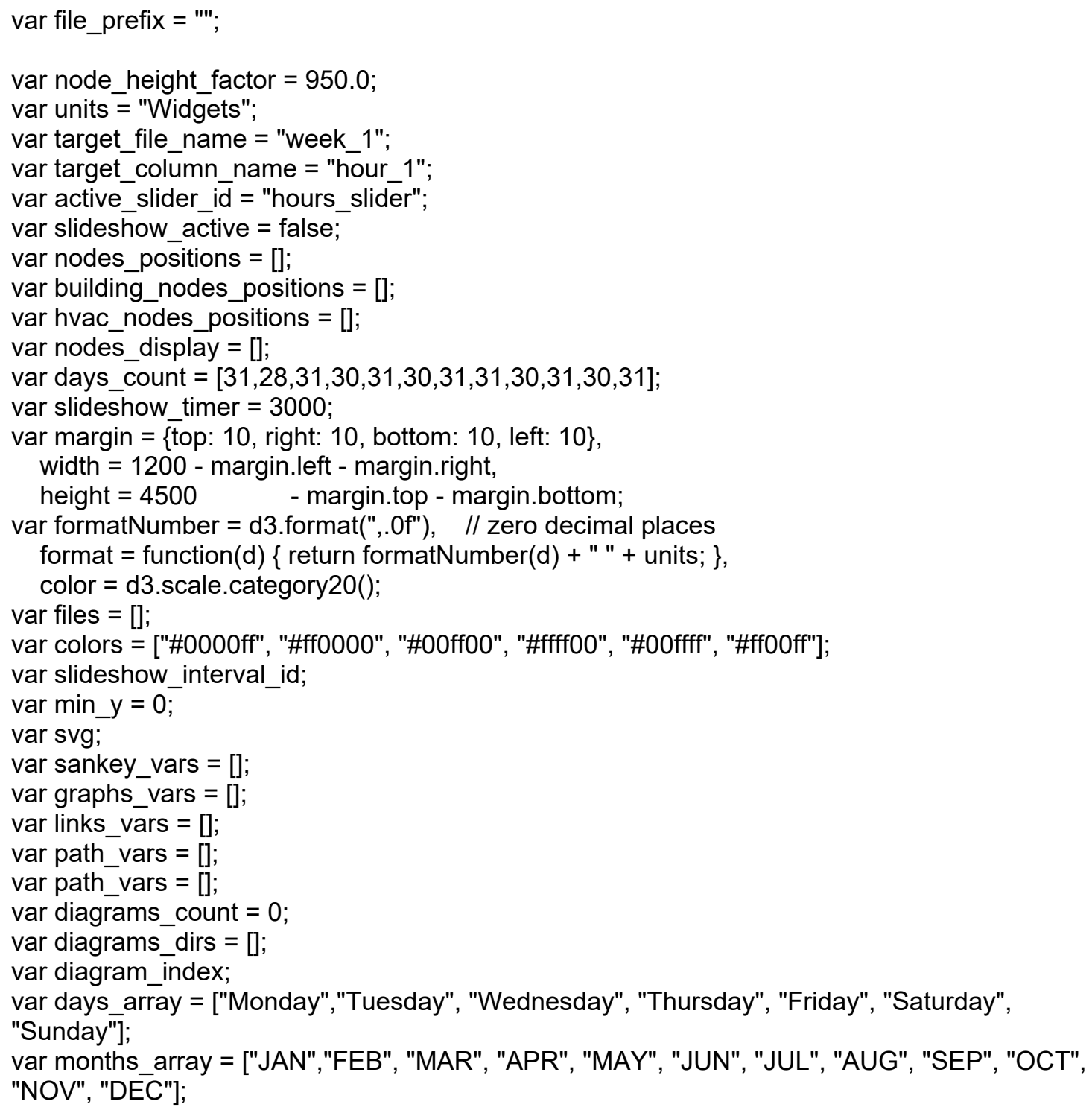




\section{Appendix E4: JavaScript code to generate any number (n) of diagrams}

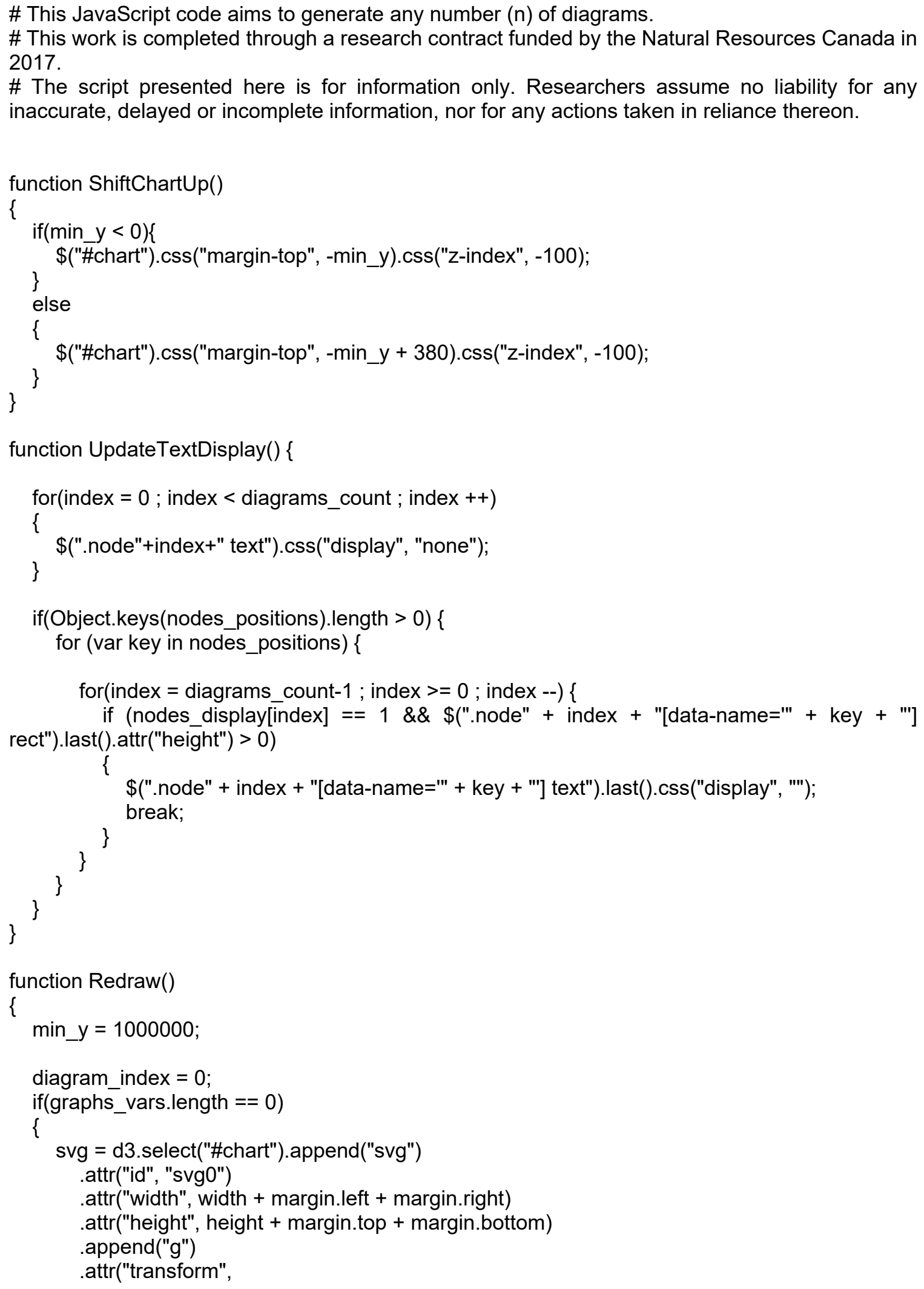




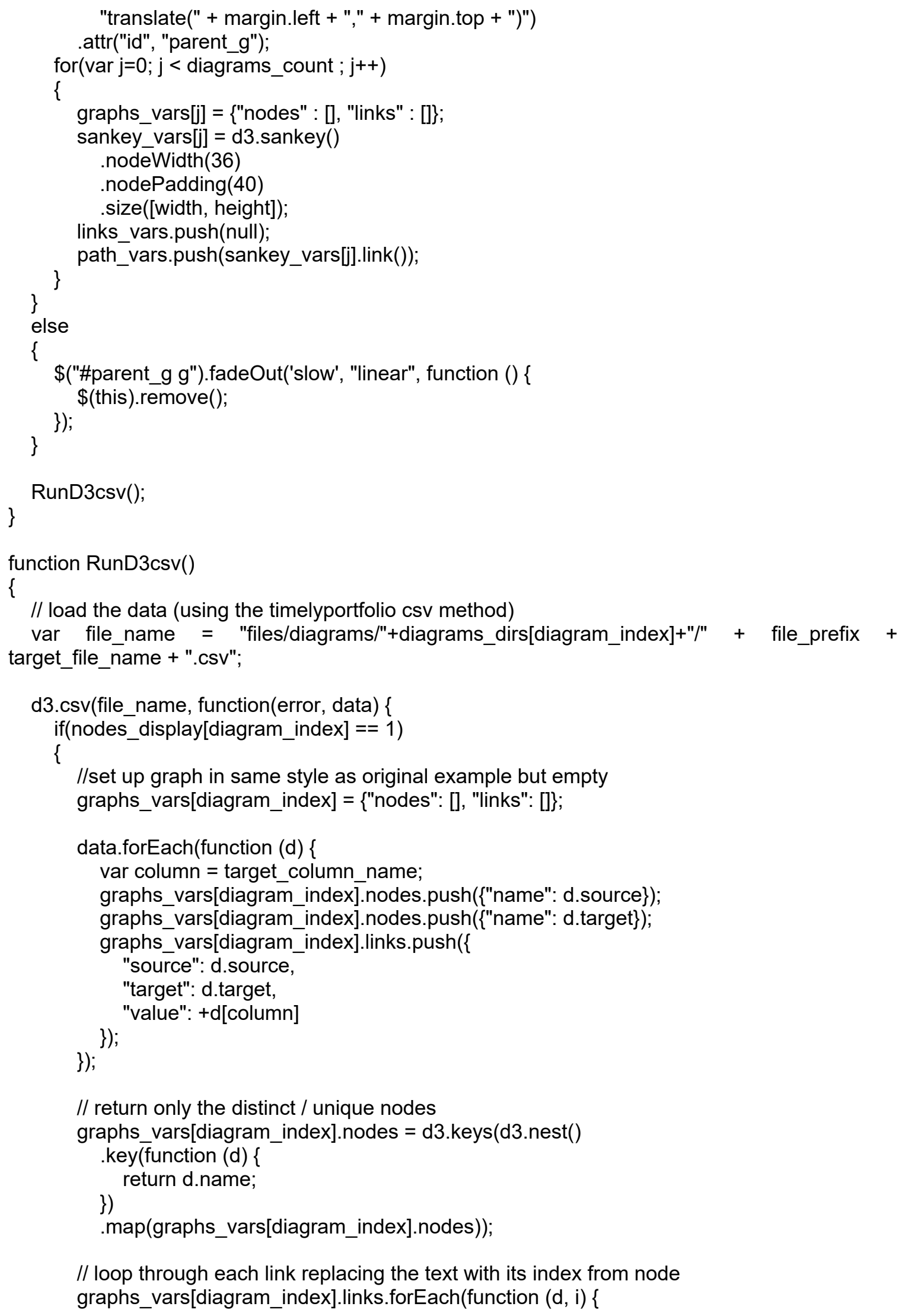


graphs_vars[diagram_index].links[i].source

graphs_vars[diagram_index].nodes.indexOf(graphs_vars[diagram_index].links[i].source); graphs_vars[diagram_index].links[i].target graphs_vars[diagram_index].nodes.indexOf(graphs_vars[diagram_index].links[i].target); \});

//now loop through each nodes to make nodes an array of objects // rather than an array of strings graphs_vars[diagram_index].nodes.forEach(function (d, i) \{ \}); graphs_vars[diagram_index].nodes[i] = $\{$ "name": d, "diagram_index": diagram_index $\}$;

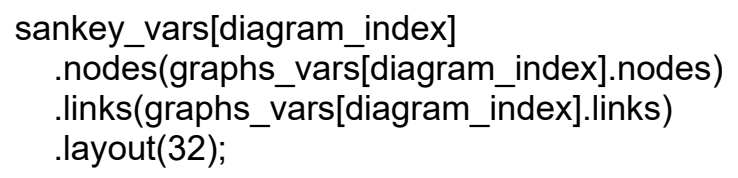




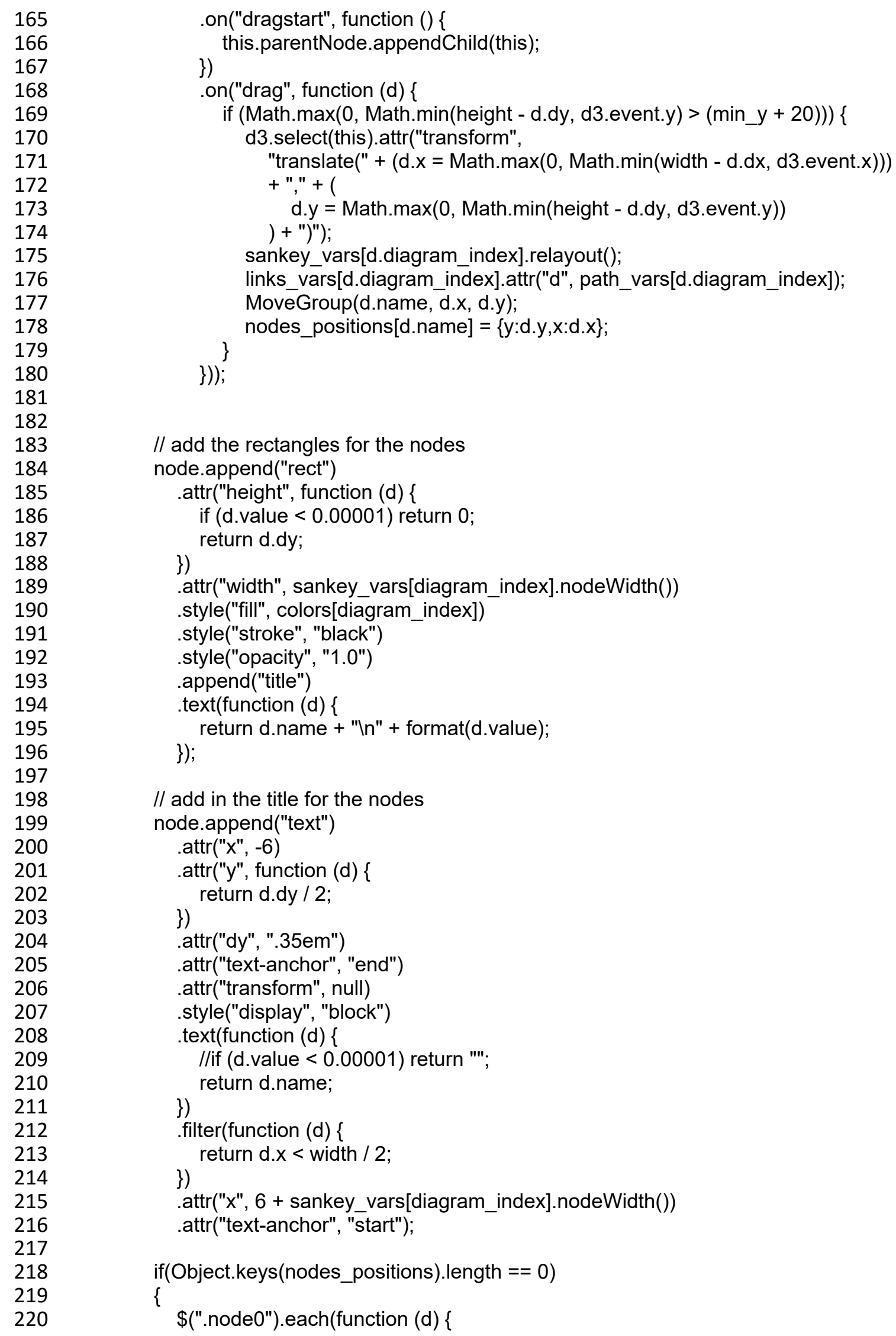




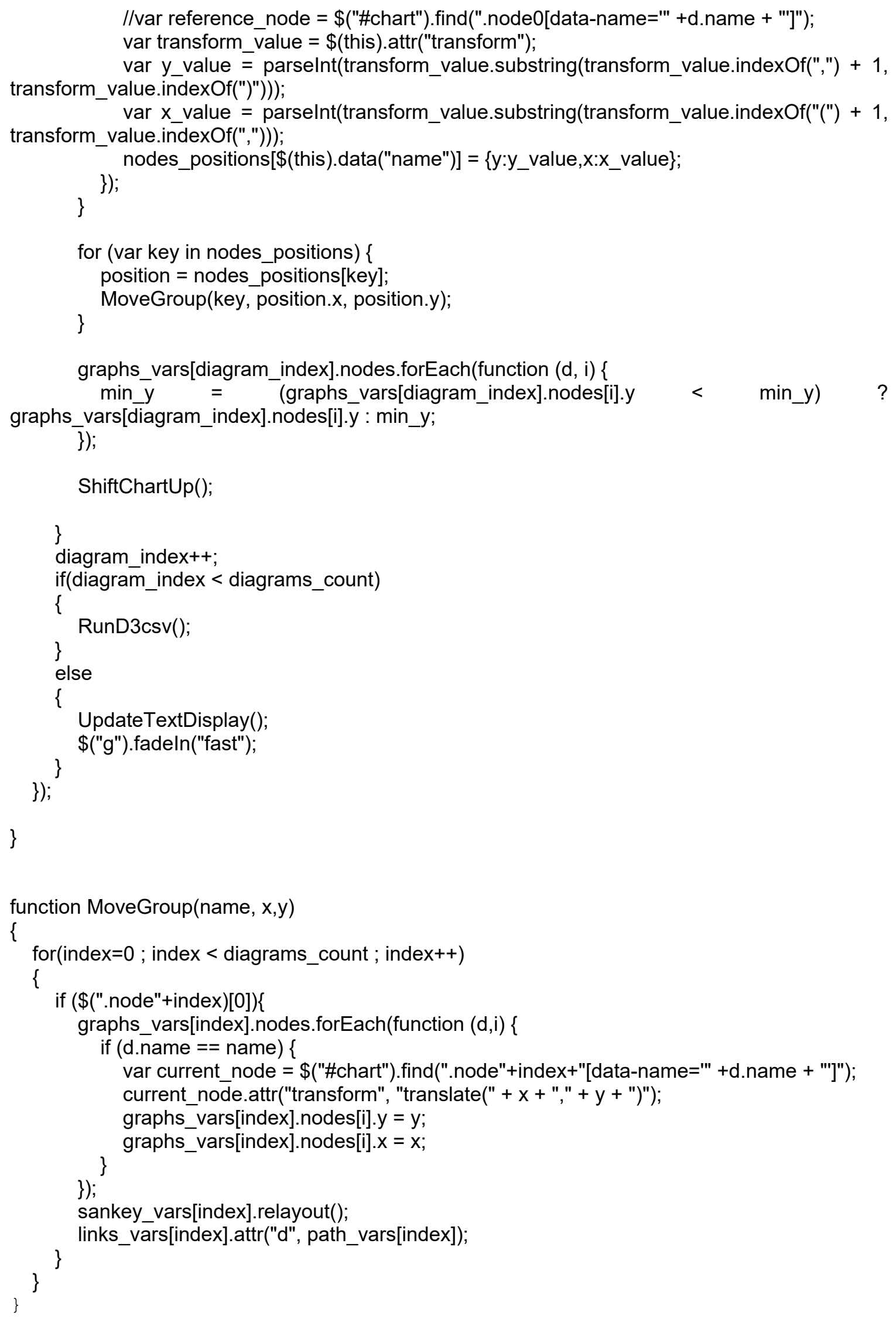




\section{Appendix E5: JavaScript code to select spatial resolution}

1 \# This JavaScript code aims to provide functions and controls to select spatial resolution.

2 \# This work is completed through a research contract funded by the Natural Resources Canada in 32017.

4 \# The script presented here is for information only. Researchers assume no liability for any 5 inaccurate, delayed or incomplete information, nor for any actions taken in reliance thereon. 


\section{Appendix E6: JavaScript code to select temporal resolution}

\# This JavaScript code aims to provide functions and controls to select temporal resolution by using slide bar.

\# This work is completed through a research contract funded by the Natural Resources Canada in 2017.

\# The script presented here is for information only. Researchers assume no liability for any inaccurate, delayed or incomplete information, nor for any actions taken in reliance thereon. 


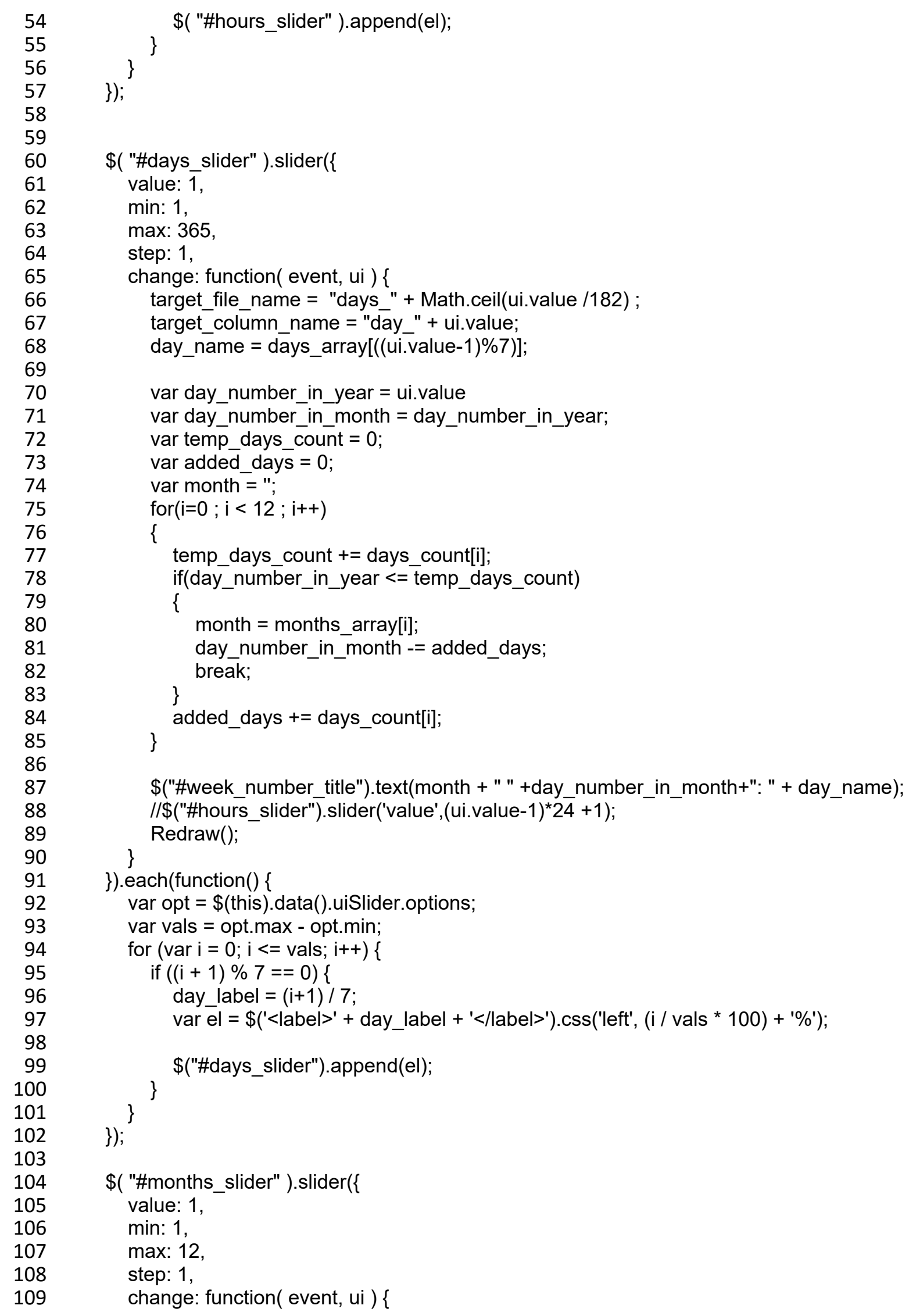




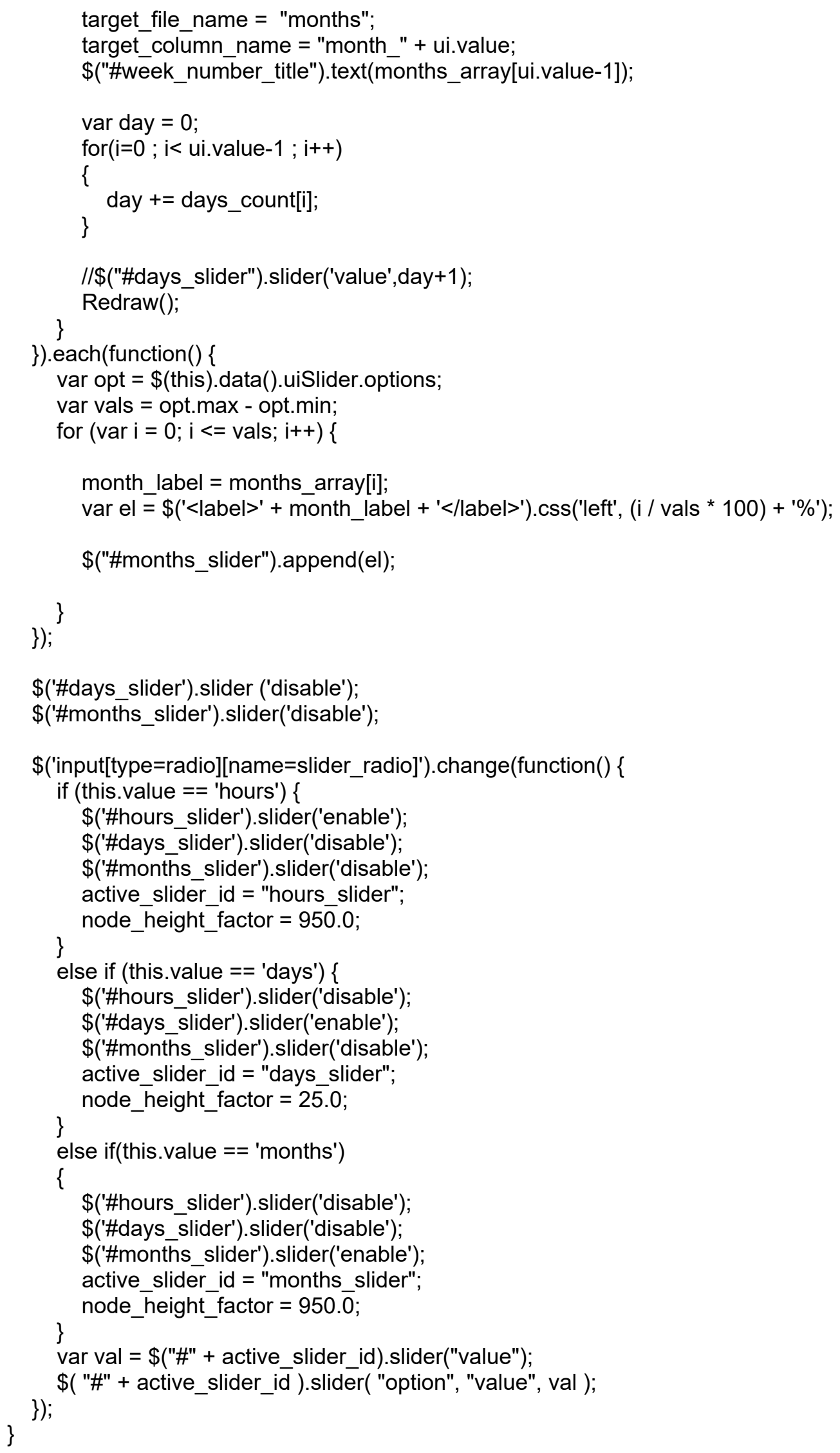




\section{Appendix E7: JavaScript code to animate the results}

10

\# This JavaScript code aims to provide functions and controls to animate the results. \# This work is completed through a research contract funded by the Natural Resources Canada in 2017.

\# The script presented here is for information only. Researchers assume no liability for any inaccurate, delayed or incomplete information, nor for any actions taken in reliance thereon.

function InitSlideShow()

\{

SetSlideshowInterval(); \}

function ToggleSlideShow()

\{

slideshow_active $=$ !(slideshow_active);

if(slideshow_active)

\{

\$("\#span_slideshow").addClass("glyphicon-pause");

\}

\$("\#span_slideshow").removeClass("glyphicon-play");

else

\{ \$("\#span_slideshow").addClass("glyphicon-play"); 


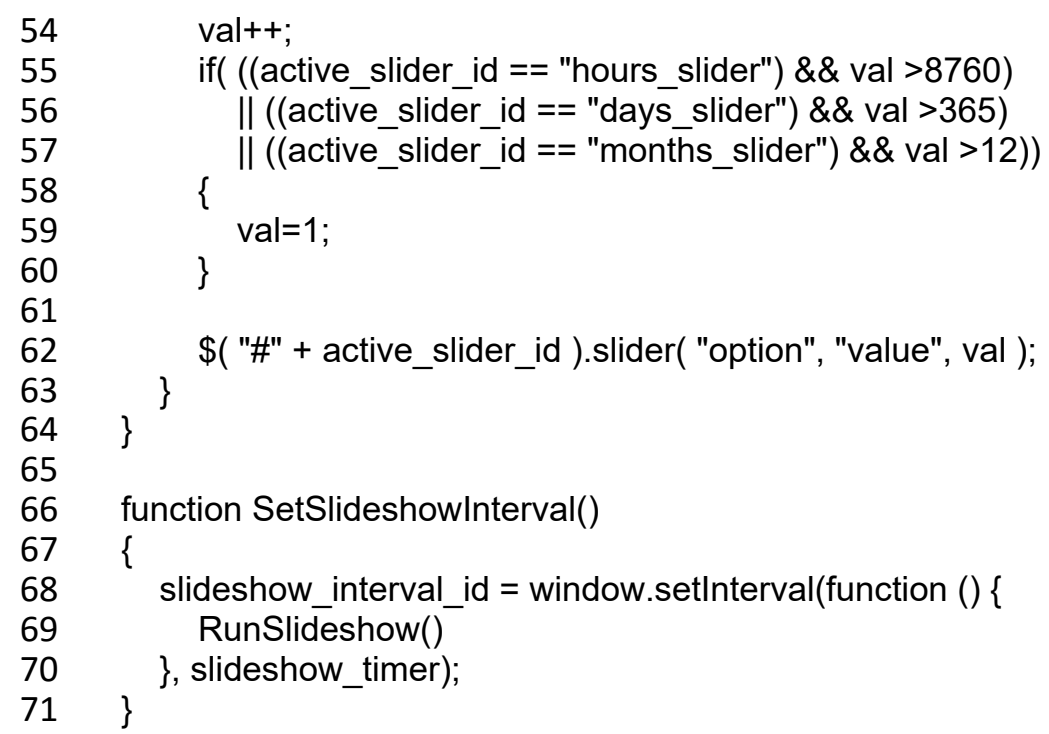




\section{Appendix E8: JavaScript code to select color for each diagram}

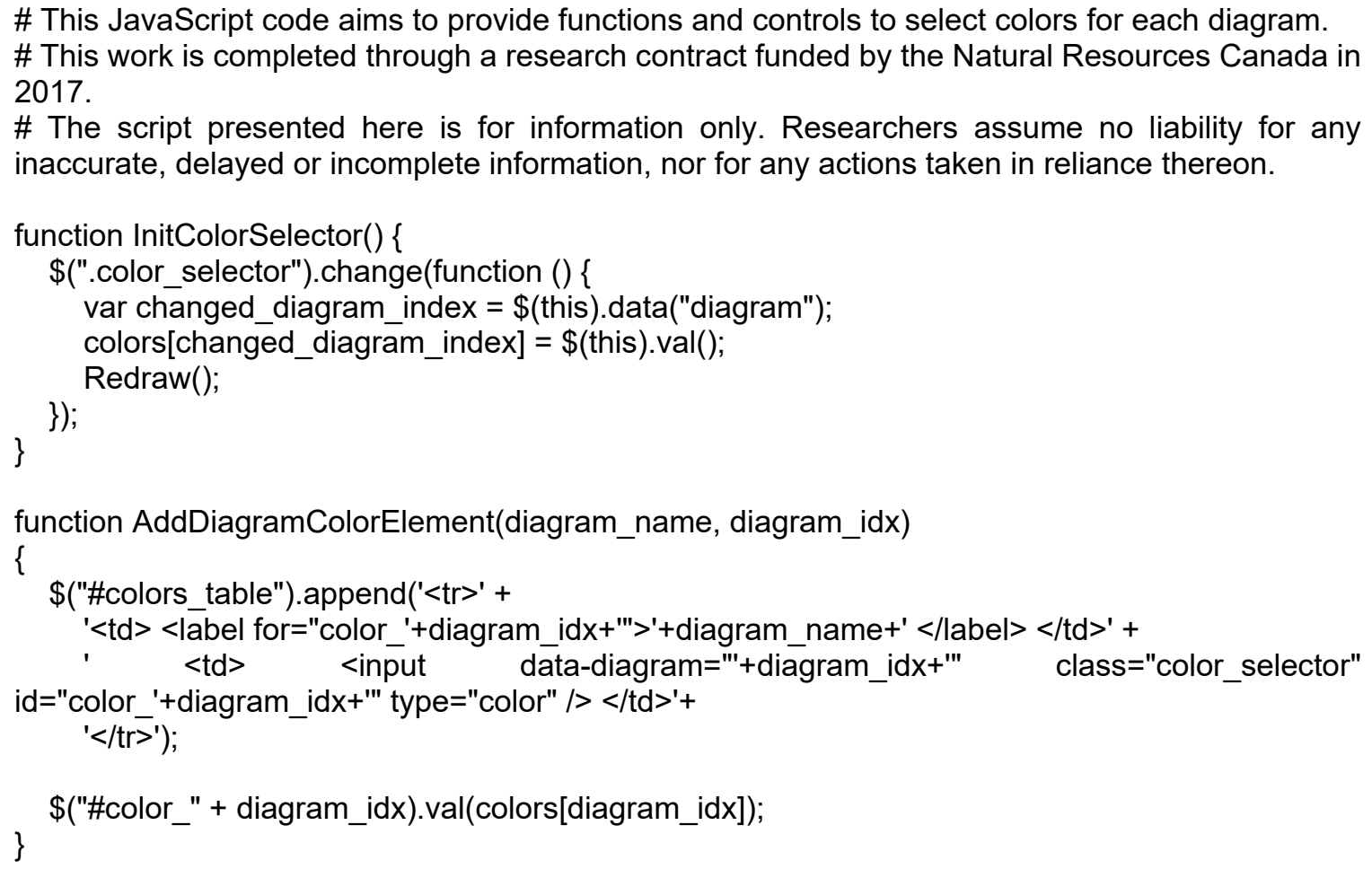




\section{Appendix E9: JavaScript code to Show/hide diagrams}

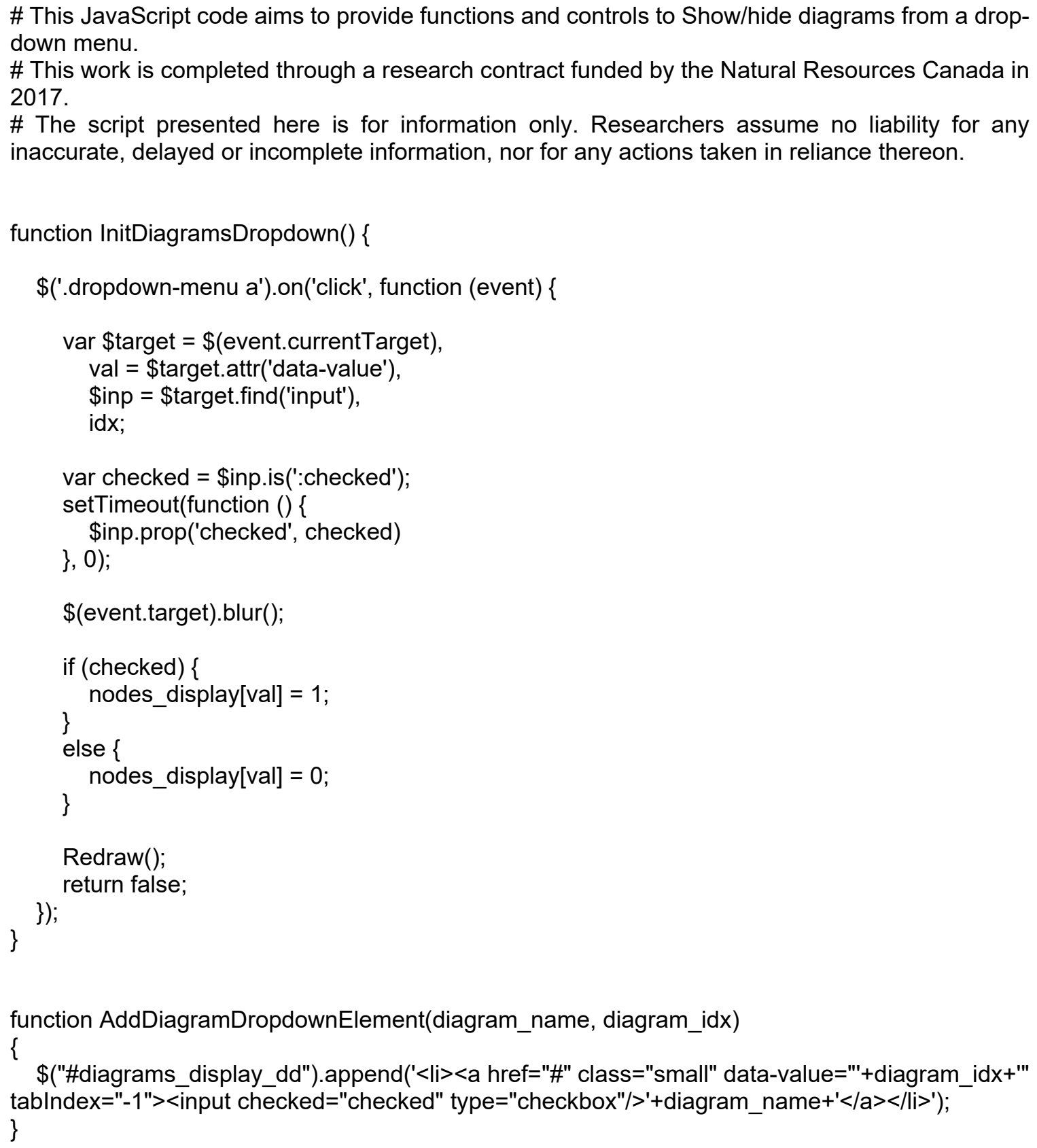




\section{Appendix E10: JavaScript code to initialize HTML elements in the web page}

29

30

31

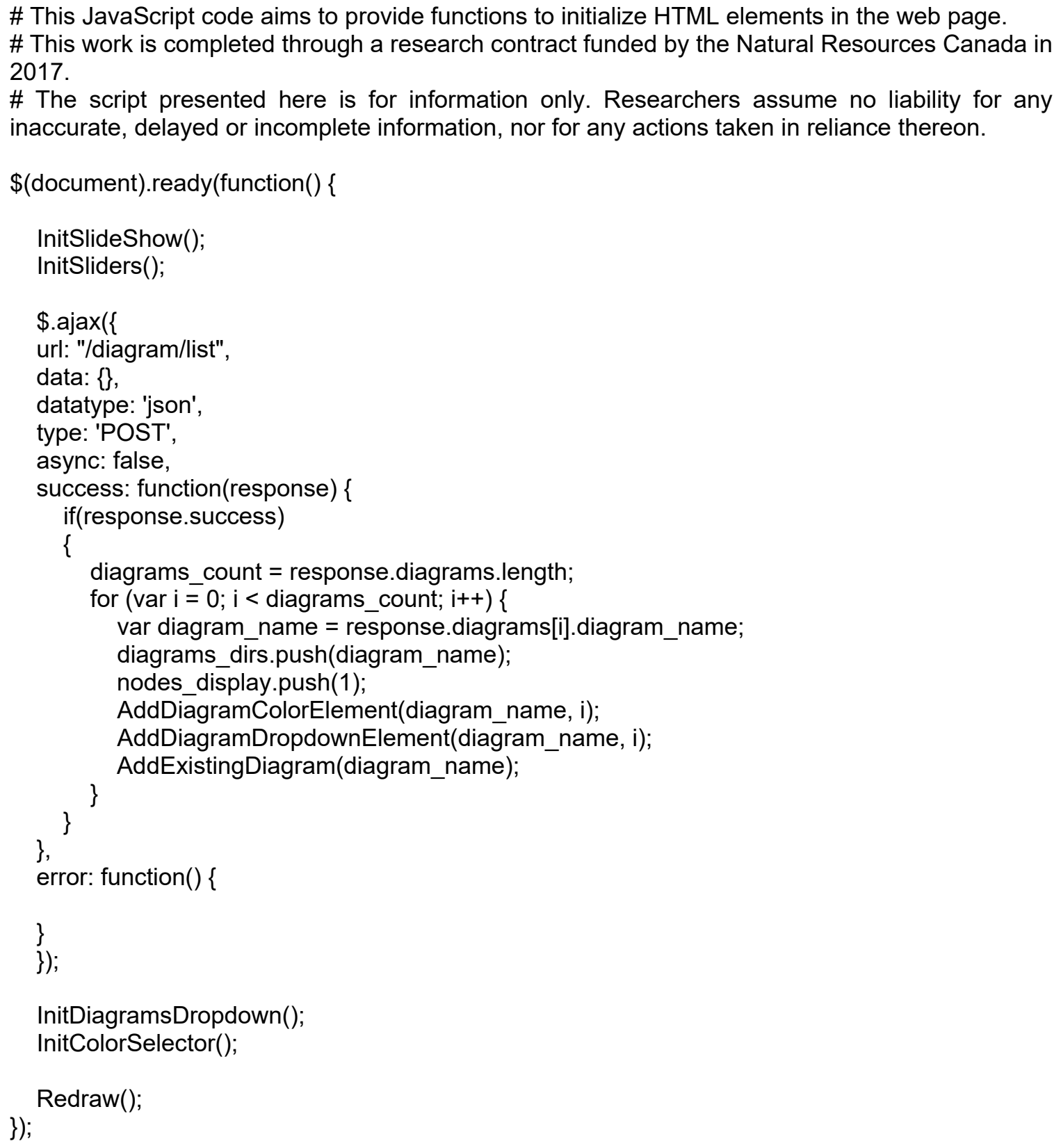




\section{Appendix F: Hypertext Preprocessor (PHP) Code}

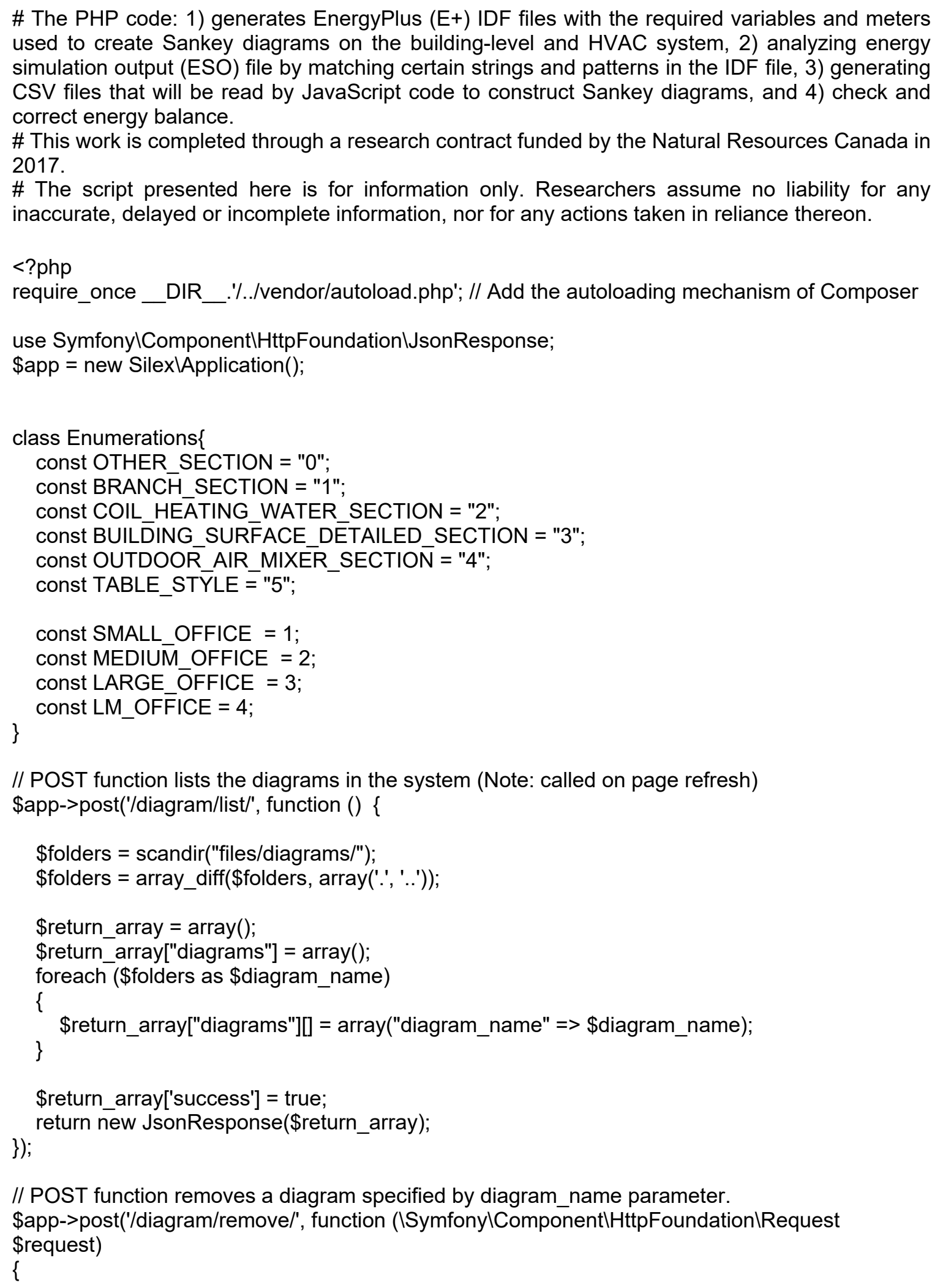




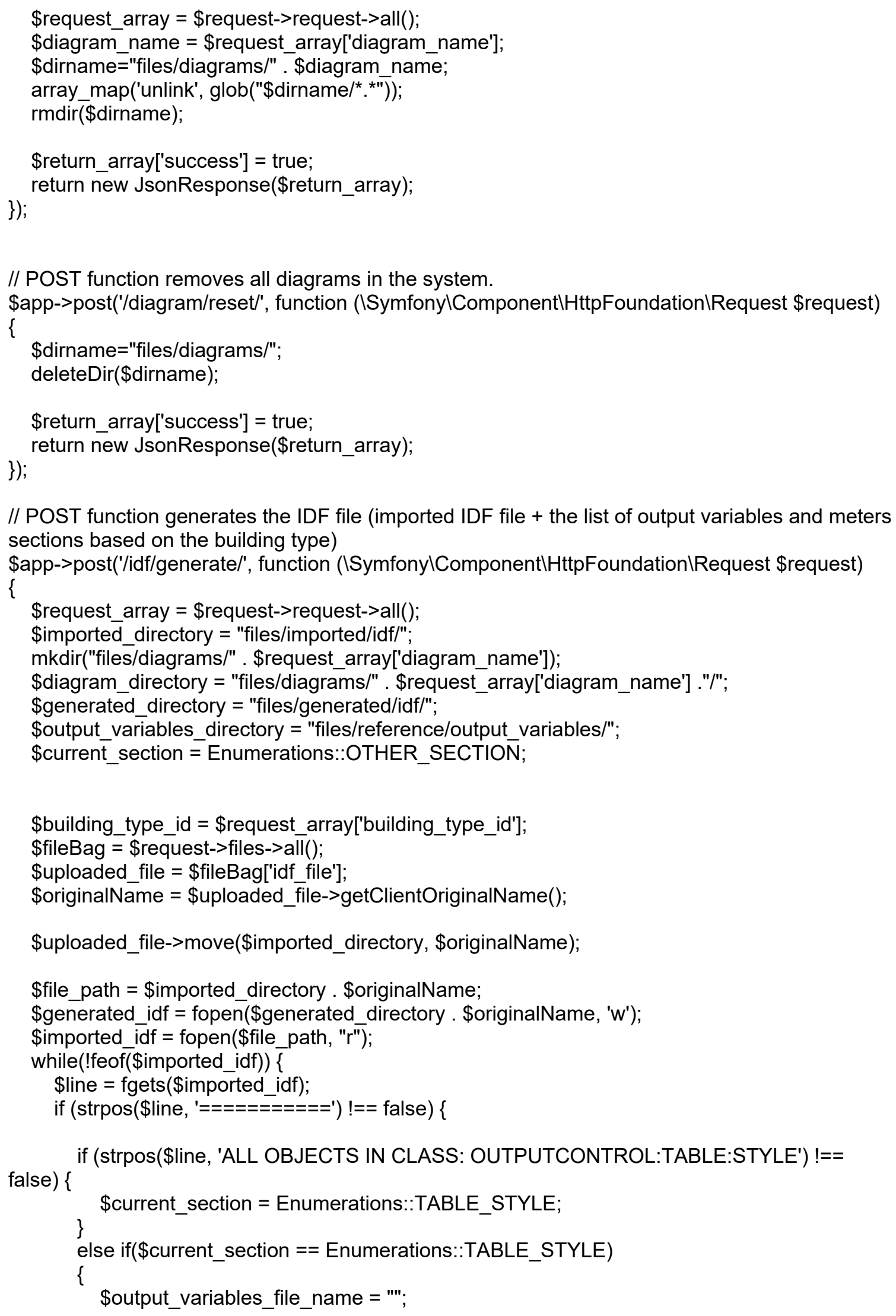




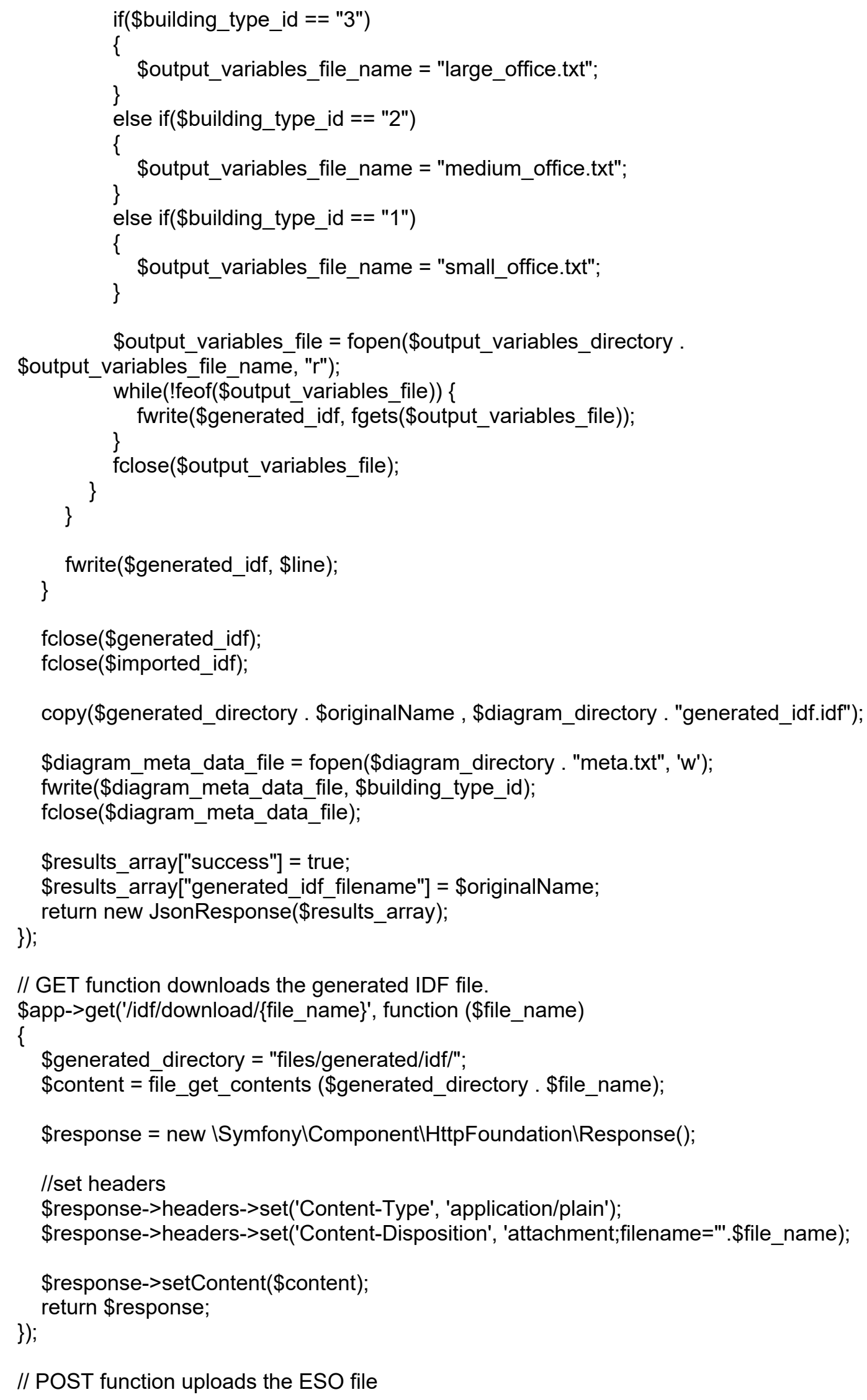


165

166

167

168

169

170

171

172

173

174

175

176

177

178

179

180

181

182

183

184

185

186

187

188

189

190

191

192

193

194

195

196

197

198

199

200

201

202

203

204

205

206

207

208

209

210

211

212

213

214

215

216

217

218

219

220

\$app->post('eso/upload/', function (ISymfonylComponentlHttpFoundation|Request \$request)

\{

\$fileBag = \$request->files->all();

foreach (\$fileBag as \$diagram_name => \$file)

\{

\$diagram_directory = "files/diagrams/" . \$diagram_name ."/";

\}

\$file->move(\$diagram_directory, "uploaded_eso.eso");

\$results_array["success"] = true;

\}$)$

return new JsonResponse(\$results_array);

// Internal function to delete directory and all its contents.

function deleteDir(\$dirPath) \{

if (! is_dir( $\$$ dirPath $)$ ) \{

if (sub throw new InvalidArgumentException("\$dirPath must be a directory");

if (substr(\$dirPath, strlen(\$dirPath) - 1, 1) != '/') \{

\} \$dirPath .= '/';

\$files = glob(\$dirPath . '*', GLOB_MARK);

foreach (\$files as \$file) \{

if (is_dir(\$file)) \{ deleteDir(\$file);

\} else \{

\} unlink(\$file);

\}

if(\$dirPath != "files/diagrams/")

\{

\}

rmdir(\$dirPath);

\}

// Internal function to get the physical unit (i.e. $\mathrm{kW}$ or $\mathrm{J}$ ) of a given eso variable.

function GetVariableUnit(\$variable_type_token)

\{

preg_match(' $\left[\left(.^{*}\right) l\right] / '$, \$variable_type_token, \$matches);

\}

return \$matches[1];

// Internal function to compare strings \$lookup_str and \$line_token ( either exact match or the \$line_token contains the \$lookup_str)

function IsLookupStringMatches(\$lookup_str, \$line_token, \$exact)

\{

if (\$exact) // exact

\{

\}

return (\$lookup_str === \$line_token); // compare strings

else

\{

\}

return (strpos(\$line_token, \$lookup_str)!== false) ;

\} 
// Internal function to check the given line (\$line_tokens) match the a lookup record (\$lookup_record)

// The main functionality of this function to check if an eso dictionary line matches some given strings in specific line tokens

// \$line_tokens -> array of the eso dictionary line data (values seperated by commas)

// \$lookup_record -> array(text to search for, line token index, row \#, exact match/not exact match, text to search for 1 , line token index 1 , exact match/not exact match) function IsLineMatches(\$line_tokens, \$lookup_record)

\section{\{}

// \$lookup_record[1] is the index of the line token that will search inside it.

// This condition is true when the given eso dictionary line has a value in the given index.

// In general, eso dictionary lines vary in number of values.

if (isset(\$line_tokens[\$lookup_record[1]]))

\{

// \$lookup_record[0] is the string to be searched for inside \$line_tokens[\$lookup_record[1]] which is the required line token.

// \$lookup_record[3] is either true or false; true means that the two given strings must match exactly, false means that the lookup string should be part of the line token if(IsLookupStringMatches(\$lookup_record[0], \$line_tokens[\$lookup_record[1]], \$lookup_record[3])) \{

// If there is no more checks on other token in the eso line, therefore, the lookup record matches the eso dictionary line.

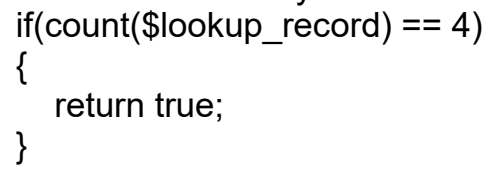

// If there is one more check on other token in the eso line, perform a similar check as above but for different line token and a another lookup string. if(count(\$lookup_record) > 4 \&\& isset(\$line_tokens[\$lookup_record[5]]))

// Internal function to read and parse the required data from the generated IDF for file for a given diagram \$diagram_name 


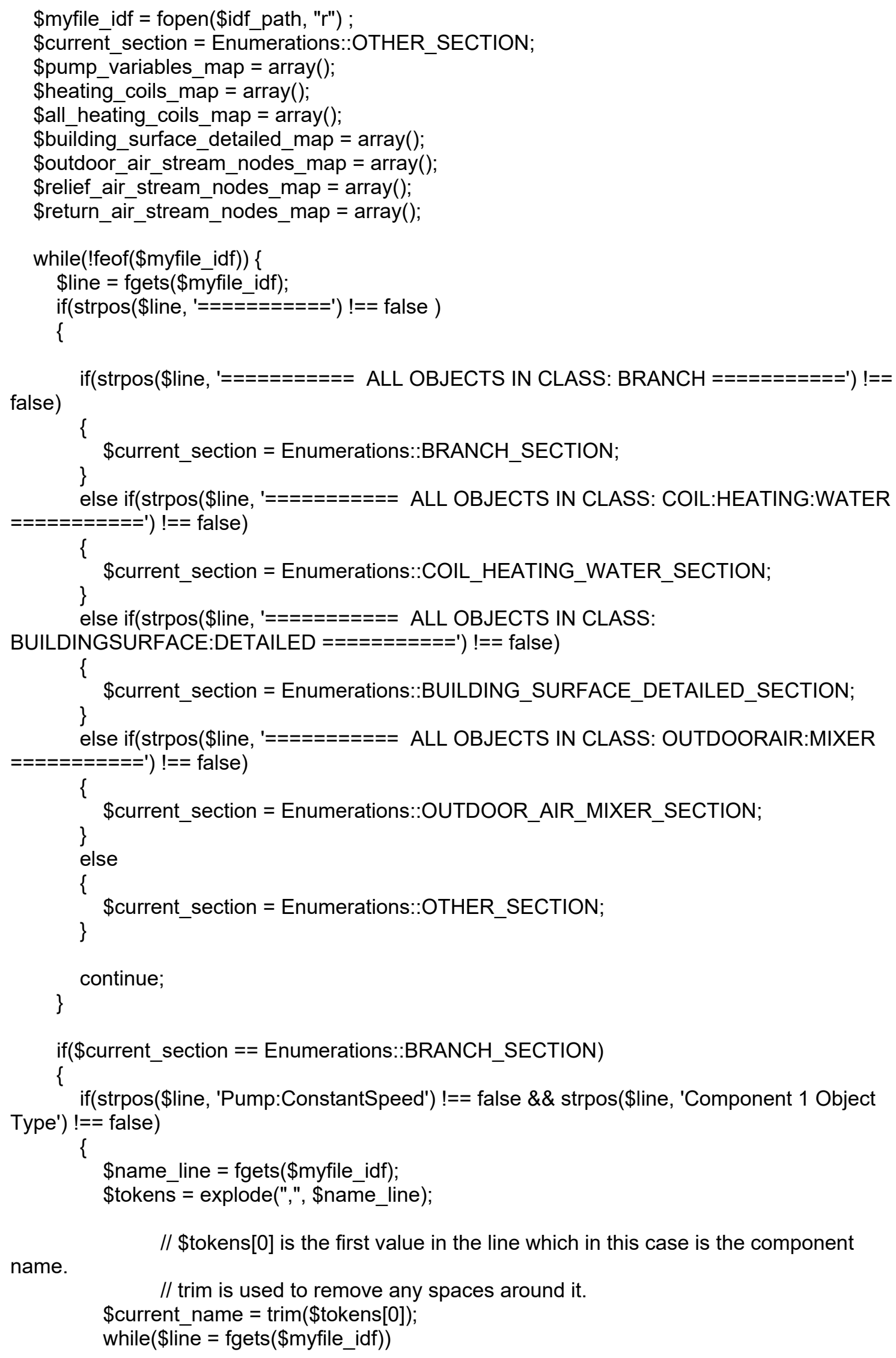




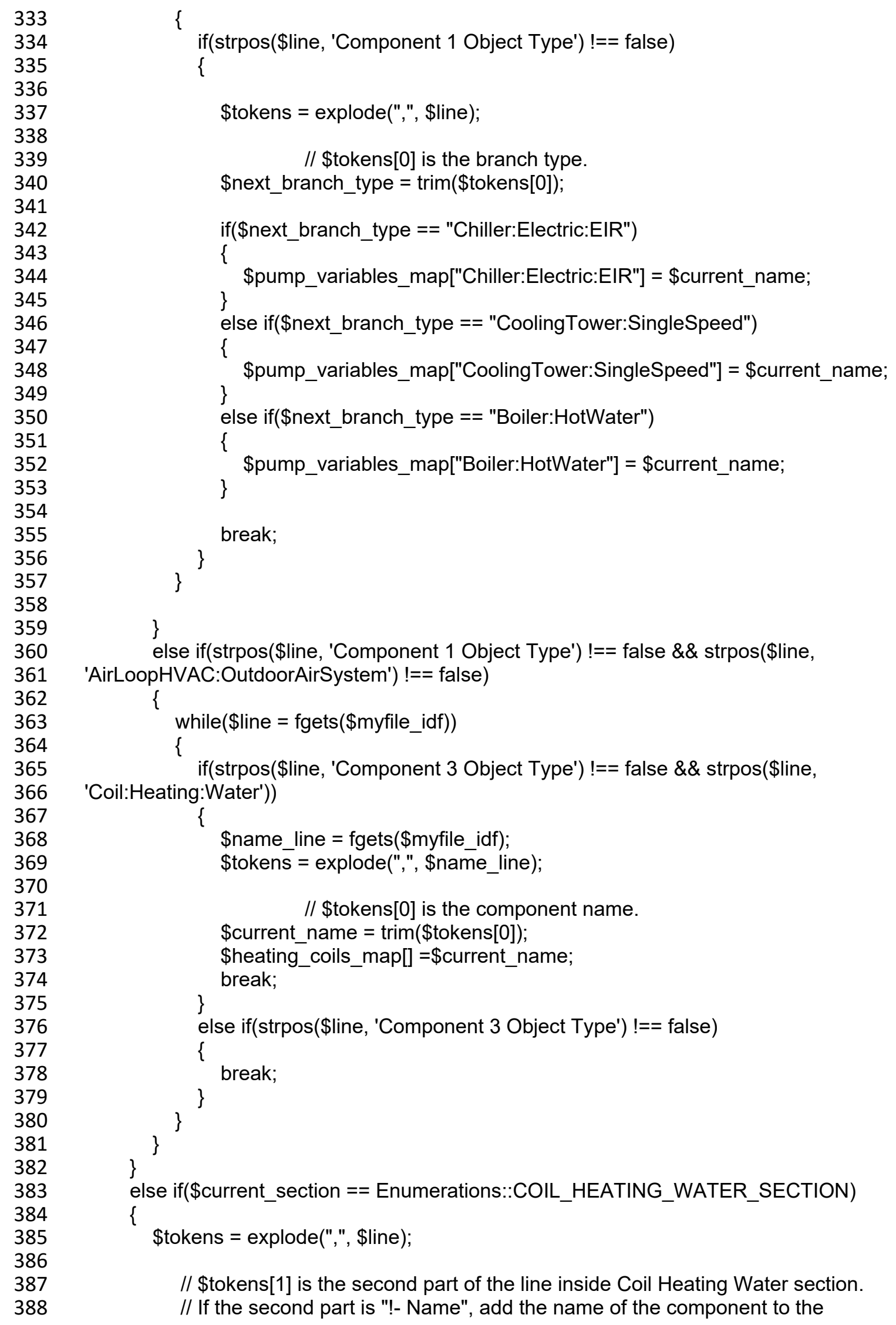


all_heating_coils_map.

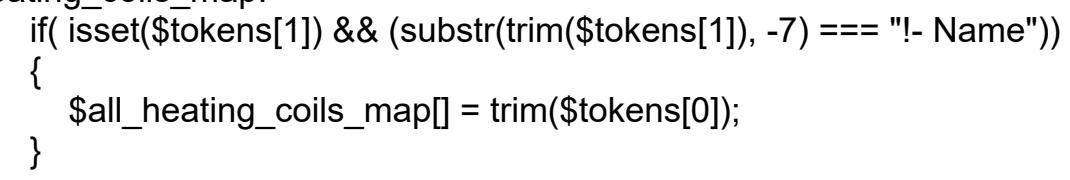




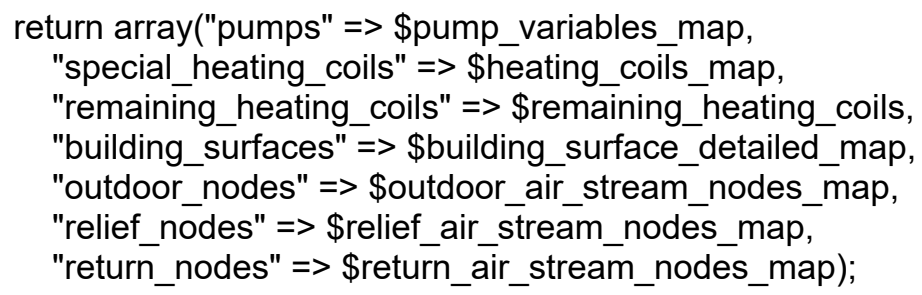

// the third value $\rightarrow(4,5,17,18,56,71,13$ means the number of rows that will be filled in the CSV files from the ESO file)

\$small_office_offset $=($ building_type $==$ Enumerations::SMALL_OFFICE) $? 1: 0$

\$direct_lookup_map = array ( array("Boiler:Heating:Gas", 2, 2, false),

// For large and medium office: Row 4 (Boiler energy transfer), Row 5 (Boiler electric energy consumption), Row 13 (AHU fans electric energy), Row 17 (lighting electric energy), Row 18 (Equipment electric energy), Row 56 (Extracted energy by cooling coils), Row 71 (Chiller electric energy)

array("Boilers:EnergyTransfer", 2, 4, false), array("Boiler Parasitic:Heating:Electricity", 2, 5, false),

one row for small office

// \$small_office_offset is 1 , which means that the number of rows are offset by array("InteriorLights:Electricity", 2, 17 - \$small_office_offset, false), 
501

502

503

504

505

506

507

508

509

510

511

512

513

514

515

516

517

518

519

520

521

522

523

524

525

526

527

528

529

530

531

532

533

534

535

536

537

538

539

540

541

542

543

544

545

546

547

548

549

550

551

552

553

554

555

556

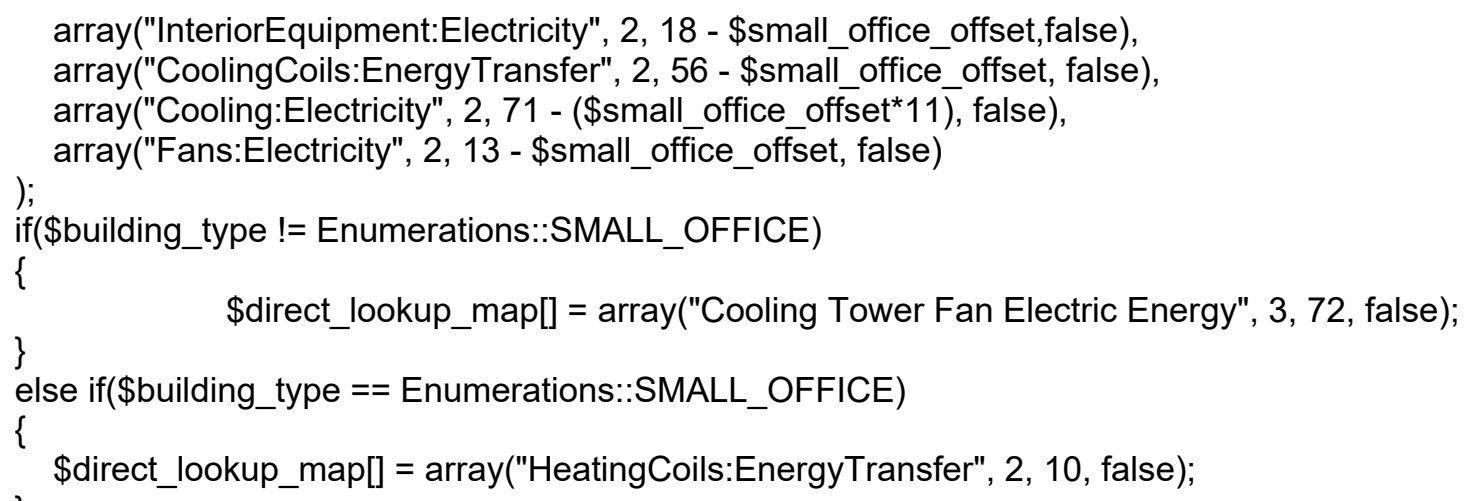

// \$indirect_lookup_map is array of records

// Record structure: (text to search for, token index, row \#, exact match/not exact match, second string to search for, second line token index, exact/non-exact match)

// The second value in each record is the line token index where a search will be performed to match the eso dictionary line against a given text to be searched for ( first value in the record)

// e.g. 2 means search inside the third token in the line.

// the third value -> $(20,29,54,30,31,32,33,34,35,36,37,45,46,47,48,14,76,77)$ means the number of rows that will be filled in the CSV files from the ESO file)

// The 5th, 6th, 7th values are similar to the first 3 values in the record but with different values to perform extra check in searching for the required line.

// For large and medium office: Row 20 (People latent energy gain), ROw 29 (Infiltration heat gain), Row 54 (Infiltration heat loss), Row 30 (Transmitted solar radiation for North window), Row 31 (Transmitted solar radiation for South window), Row 32 (Transmitted solar radiation for East window), Row 33 (Transmitted solar radiation for West window), Row 34 (Heat gain from North window), Row 35 (Heat gain from South window), Row 36 (Heat gain from East window), Row 37 (Heat gain from West window), Row 45 (Heat loss from North window), Row 46 (Heat loss from South window), Row 47 (Heat loss from East window), Row 48 (Heat loss from West window), Row 14 (Humidifier energy consumption), Row 76 (Surface Heat Storage loss Rate) Row 77 (Surface Heat Storage Gain Rate)

// \$small_office_offset is 1, which means that the number of rows are offset by one row for small office CSV file.

// The last two records are different because the Small office is offset by 14 rows in the

\$indirect_lookup_map $=$ array $($ array("People Sensible Heating Energy", 3, 19 \$small_office_offset, false), array("People Latent Gain Energy", 3, 20 - \$small_office_offset, false), array("Zone Infiltration Total Heat Gain Energy", 3, 29 - \$small_office_offset, false), array("Zone Infiltration Total Heat Loss Energy", 3, 54 - \$small_office_offset, false), array("Zone Windows Total Transmitted Solar Radiation Rate", 3, 30 - \$small_office_offset, false, "NORTH", 2,false),

array("Zone Windows Total Transmitted Solar Radiation Rate", 3, 31 - \$small_office_offset, false, "SOUTH", 2,false), array("Zone Windows Total Transmitted Solar Radiation Rate", 3, 32 - \$small_office_offset, false, "EAST", 2,false), array("Zone Windows Total Transmitted Solar Radiation Rate", 3, 33 - \$small_office_offset, false, "WEST", 2,false), 2 ,false), array("Zone Windows Total Heat Gain Rate", 3, 34 - \$small_office_offset, false, "NORTH", 2,false), array("Zone Windows Total Heat Gain Rate", 3, 35 - \$small_office_offset,false, "SOUTH", 

2 ,false)

array("Zone Windows Total Heat Gain Rate", 3, 36 - \$small_office_offset,false, "EAST", array("Zone Windows Total Heat Gain Rate", 3, 37 - \$small_office_offset,false, "WEST", 2,false), 2,false), array("Zone Windows Total Heat Loss Rate", 3, 45 - \$small_office_offset,false, "NORTH", array("Zone Windows Total Heat Loss Rate", 3, 46 - \$small_office_offset,false, "SOUTH", 2,false), array("Zone Windows Total Heat Loss Rate", 3, 47 - \$small_office_offset,false, "EAST", 2,false), 2,false), array("Zone Windows Total Heat Loss Rate", 3, 48 - \$small_office_offset,false, "WEST", array("Air System Humidifier Gas Energy", 3, 14 - \$small_office_offset, false), array("Surface Heat Storage Loss Rate", 3, 76 - (\$small_office_offset*14), false), ); array("Surface Heat Storage Gain Rate", 3, 77 - (\$small_office_offset*14), false)

\$idf_data = getldfData $($ diagram_name $)$;

// Pump variables

// For large, medium, and small office: Row 63 (Chilled water (CHW) pump electric energy), Row 64 (CHW pump frictional loss), Row 61 (Condensing water (CDW) electric energy), Row 62 (CDW pump frictional losses), Row 6 (Hot water (HW) pump electric energy), Row 7 (HW pump frictional loss)

foreach(\$idf_data["pumps"] as \$pump_type => \$variable_name) \{

if(\$pump_type == "Chiller:Electric:EIR" \&\& \$building_type != Enumerations::SMALL_OFFICE)

\{

\$direct_lookup_map[] = array(strtoupper(\$variable_name), 2, 63, true, "Pump Electric Energy", 3, false);

\$direct_lookup_map[] = array(strtoupper(\$variable_name), 2, 64, true, "Pump Fluid Heat Gain Energy", $\overline{3}$, false);

\}

else if(\$pump_type == "CoolingTower:SingleSpeed" \&\& \$building_type != Enumerations::SMĀLL_OFFICE)

\{

\$direct_lookup_map[] = array(strtoupper(\$variable_name), 2, 61, true, "Pump Electric Energy", 3, false);

\$direct_lookup_map[] = array(strtoupper(\$variable_name), 2, 62, true, "Pump Fluid Heat Gain Energy", 3 , false);

\$direct_lookup_map[] = array(strtoupper(\$variable_name), 2, 6, true, "Pump Electric Energy", 3, false);

\$direct_lookup_map[] = array(strtoupper(\$variable_name), 2, 7, true, "Pump Fluid Heat Gain Energy", 3 , false);

if(!empty(\$idf_data['special_heating_coils']) \&\& \$building_type != Enumerations::S̄MALL_OFFIC̄E)

\{ // For large, medium, and small office: Row 10 (Energy consumption by heating coils), Row 
613

614

615

616

617

618

619

620

621

622

623

624

625

626

627

628

629

630

631

632

633

634

635

636

637

638

639

640

641

642

643

644

645

646

647

648

649

650

651

652

653

654

655

656

657

658

659

660

661

662

663

664

665

666

667

668

11 (VAV-Reheat energy consumption)

\}

\$indirect_lookup_map[] = array $($ \$idf_data['special_heating_coils'], 2, 10, true);

if(!empty(\$idf_data['remaining_heating_coils']) \&\& \$building_type !=

Enumerations::S̄MALL_OFFICE)

\{

\}

\$indirect_lookup_map[] = array $($ \$idf_data['remaining_heating_coils'], 2, 11, true);

\$walls_variables = array () ;

\$floors_variables $=$ array () ;

\$ceilings_variables = array () ;

foreach (\$idf_data['building_surfaces'] as \$building_surface_variable)

\{

if(stripos(\$building_surface_variable, "Wall") !== false)

\{

\}

\$walls_variables[] = strtoupper(\$building_surface_variable);

else if(stripos(\$building_surface_variable, "Floor") !== false)

\{

\$floors_variables[] = strtoupper(\$building_surface_variable);

\}

else if(stripos(\$building_surface_variable, "Ceiling") !== false)

\{

\}

\$ceilings_variables[] = strtoupper(\$building_surface_variable);

\}

// For large and medium office: Row 41 (Heat gain from walls), Row 42 (Heat gain from roofs), Row 43 (Heat gain from floors), Row 50 (Heat loss from walls), Row 51 (heat loss from floors), Row 52 (heat loss from roofs)

// \$small_office_offset is 1, which means that the number of rows are offset by one row for small office

\$small_office_offset $=($ \$building_type $==$ Enumerations::SMALL_OFFICE) $? 1: 0$;

\$indirect_lookup_map[] = array(\$walls_variables, 2, 41 - \$small_office_offset, true, "Surface Inside Face Conduction Heat Gain Rate", 3, false);

\$indirect_lookup_map[] = array(\$ceilings_variables, 2, 42 - \$small_office_offset, true, "Surface Inside Face Conduction Heat Gain Rate", 3, false);

\$indirect_lookup_map[] = array(\$floors_variables, 2, 43 - \$small_office_offset, true, "Surface Inside Face Conduction Heat Gain Rate", 3, false);

\$indirect_lookup_map[] = array(\$walls_variables, 2, 50 - \$small_office_offset, true, "Surface Inside Face Conduction Heat Loss Rate", 3, false);

\$indirect_lookup_map[] = array(\$ceilings_variables, 2, 51 - \$small_office_offset, true, "Surface Inside Face Conduction Heat Loss Rate", 3, false);

\$indirect_lookup_map[] = array(\$floors_variables, 2, 52 - \$small_office_offset, true, "Surface Inside Face Conduction Heat Loss Rate", 3, false);

\$complex_lookup_map = array ();

\$complex_lookup_map[] = array(\$idf_data['outdoor_nodes'], 2, 16 - \$small_office_offset, true);

\$complex_lookup_map[] = array(\$idf_data['relief_nodes'], 2, 55 - \$small_office_offset, true);

\$hvac_complex_lookup_map = array ();

\$hvac_complex_lookup_map[] = array(\$idf_data['return_nodes'], 2, 16, true); 


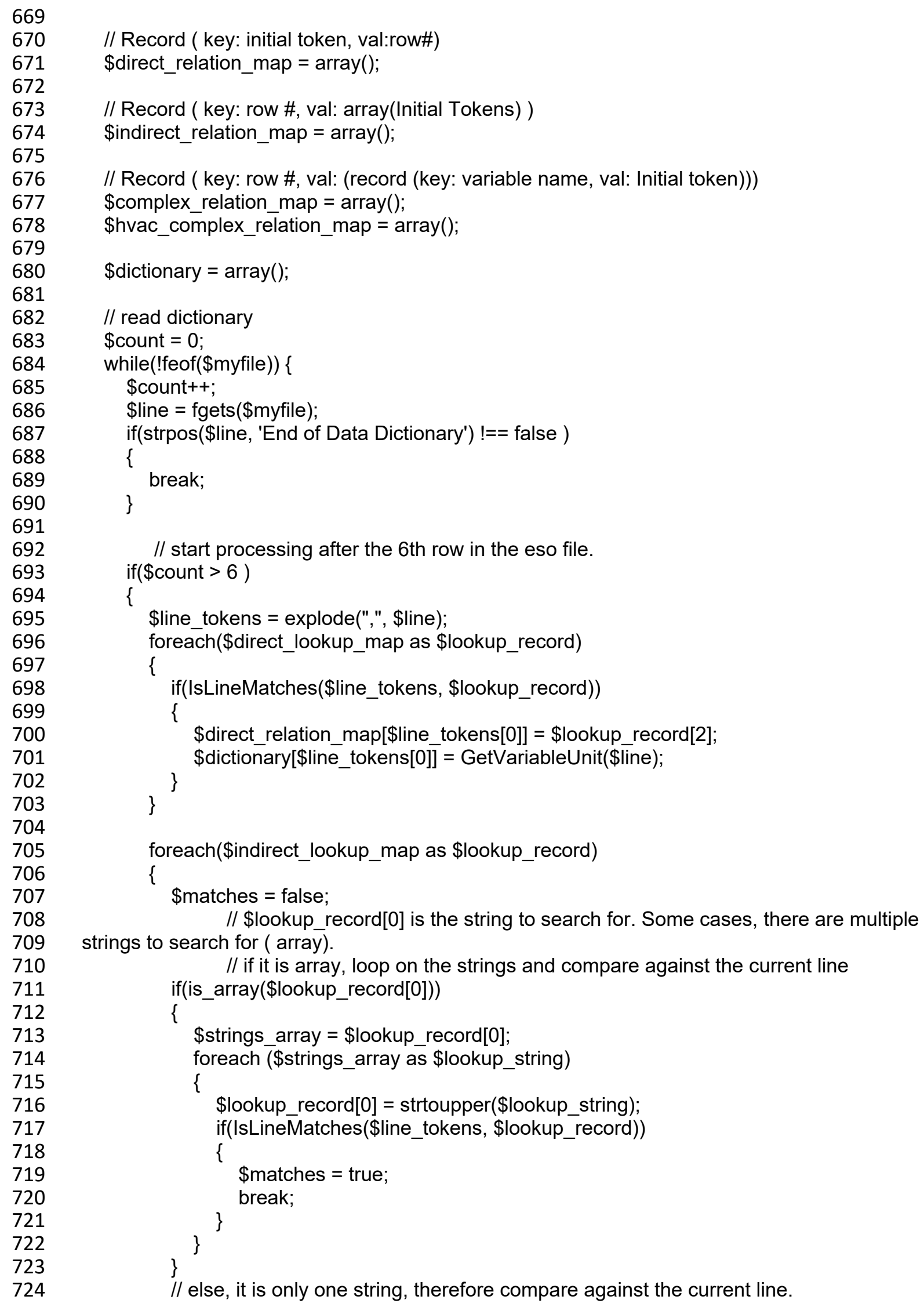




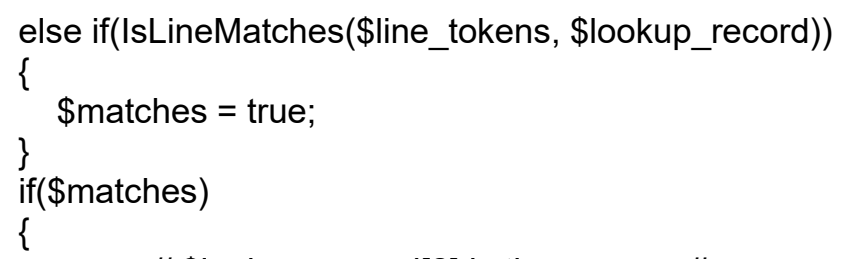




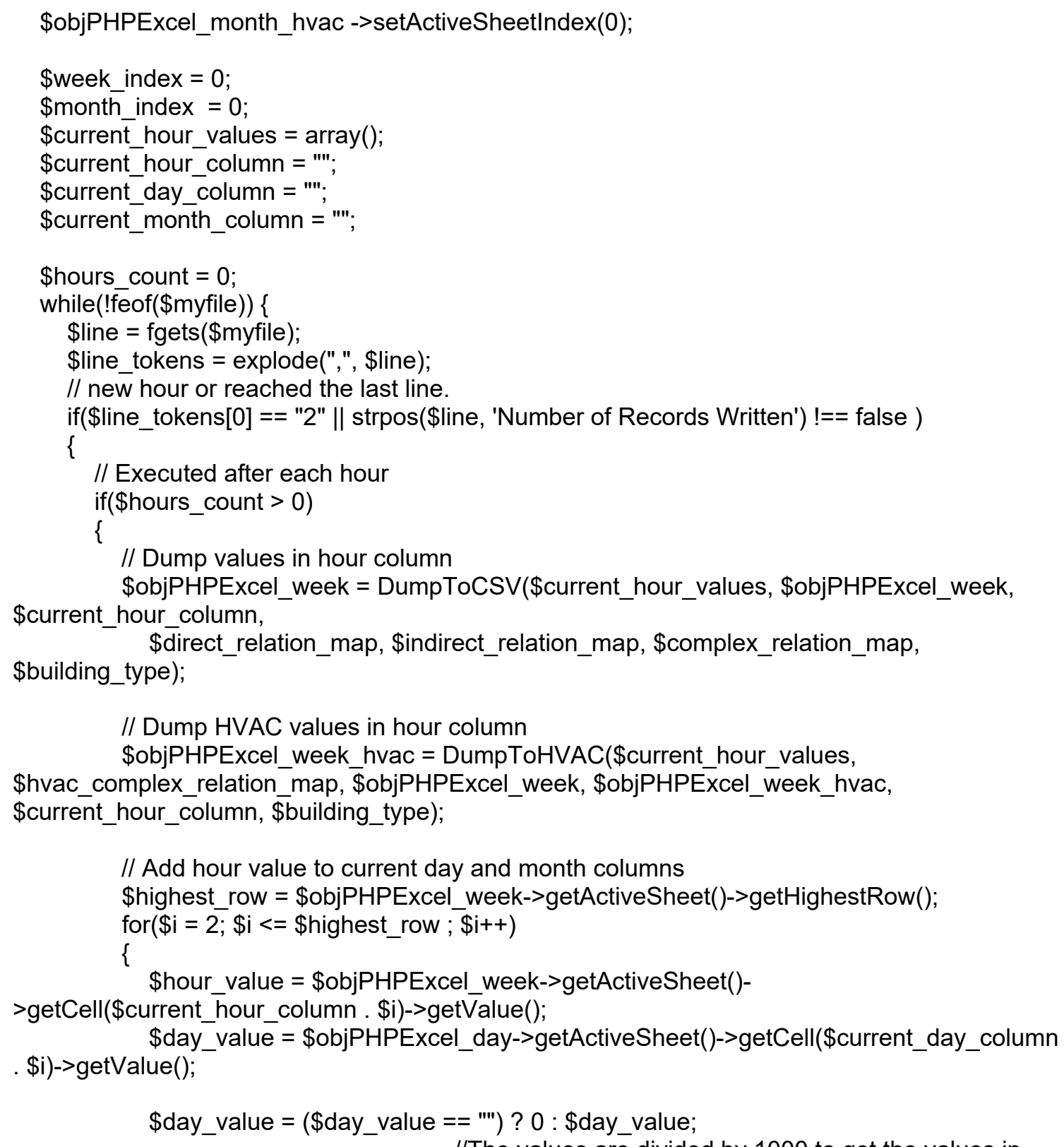


// Add hour HVAC value to current day and month columns

\$highest_row_hvac = \$objPHPExcel_week_hvac->getActiveSheet()->getHighestRow(); for $(\$ i=2 ; \$ i<=\$$ highest_row_hvac; $\$ \mathrm{i}++)$

\{

\$hour_value $=$ \$objPHPExcel_week_hvac->getActiveSheet()-

$>$ getCell(\$current_hour_column . \$i)->getValue();

\$day_value $=$ \$objPHPExcel_day_hvac->getActiveSheet()-

$>$ getCell(\$current_day_column . \$i)->getValue ();

\$day_value $=($ day_value $==$ "'") $? 0:$ day_value; //The values are divided by 1000 to get the values in

kWh

\$i, \$day_value);

\$day_value $+=($ Shour_value / 1000.0);

\$objPHPExcel_day_hvac->getActiveSheet()->setCellValue(\$current_day_column .

\$month_value = \$objPHPExcel_month_hvac->getActiveSheet()>getCell(\$current_month_column . \$i)->getValue();

\$month_value $=($ \$month_value $==" ')$ ? $0:$ \$month_value;

$\mathrm{kWh}$

//The values are divided by 1000 to get the values in

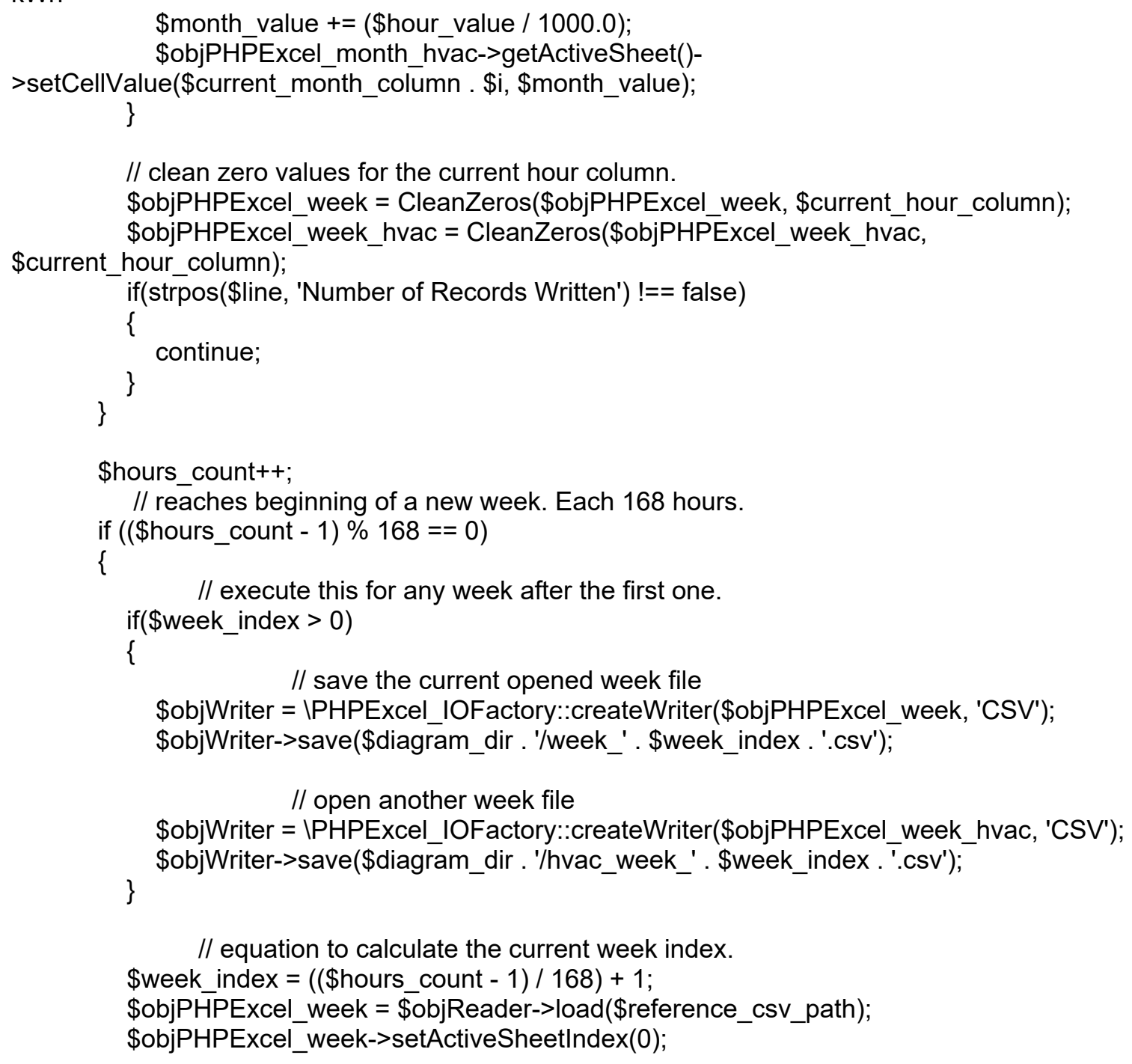


893

894

895

896

897

898

899

900

901

902

903

904

905

906

907

908

909

910

911

912

913

914

915

916

917

918

919

920

921

922

923

924

925

926

927

928

929

930

931

932

933

934

935

936

937

938

939

940

941

942

943

944

945

946

947

948

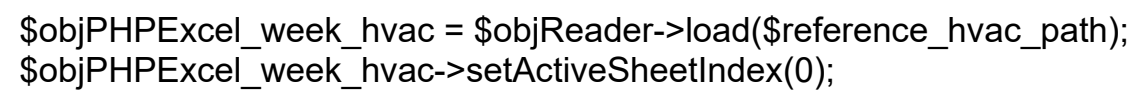

// \$line_tokens[1] is the day value from eso record. \$record_day $=\$$ line_tokens[1];

// reaches the beginning of a new day. each 24 hours. if $((\$$ hours_count - 1) \% $24==0)$

\{

// clean 0's of the current day columns

// execute this for any day except the first day.

if $($ \$record_day $>1)$

\{

\$objPHPExcel_day = CleanZeros(\$objPHPExcel_day, \$current_day_column);

\$objPHPExcel_day_hvac $=$ CalculateHvacEnergyFlows $($ \$objPHPExcel_day_hvac, \$current_day_column, \$building_type, \$objPHPExcel_day);

\$current_day_column);

\$objPHPExcel_day_hvac = CleanZeros $($ \$objPHPExcel_day_hvac,

// open new file at the begining of the year and at the mid year.

// There are two files for the days ( days_1, days_2). days_1 -> first 183 days in

the year. days_2 -> the remaining 182 days in the year.

if $($ \$record_day $==1 \|$ \$record_day $==183$ )

\{

// save the first days file

if $($ \$record_day $==183$ )

\{

\$objPHPExcel_day = CleanZeros(\$objPHPExcel_day, \$current_day_column);

\$objWriter = IPHPExcel_IOFactory::createWriter(\$objPHPExcel_day, 'CSV');

\$objWriter->save(\$diagram_dir . '/days_1.csv');

\$objPHPExcel_day_hvac $=$ CalculateHvacEnergyFlows $($ \$objPHPExcel_day_hvac, \$current_day_column, \$building_type, \$objPHPExcel_day);

\$current_day_column);

\$objPHPExcel_day_hvac =CleanZeros $($ \$objPHPExcel_day_hvac,

'CSV');

$$
\text { \}\$objWriter->save(\$diagram_dir . '/hvac_days_1.csv'); }
$$

\$objWriter = IPHPExcel_IOFactory::createWriter(\$objPHPExcel_day_hvac,

// open new days files

\$objPHPExcel_day = \$objReader->load (\$reference_csv_path);

\$objPHPExcel_day->setActiveSheetIndex(0);

\$objPHPExcel_day_hvac = \$objReader->load $($ \$reference_hvac_path); \}

\$objPHPExcel_day_hvac->setActiveSheetIndex(0);

index

// set the column index using relationship between day index in eso file and the column

\$column_index $=$ \$record_day +1 ;

// for the days_2 file, subtract the offset 182 days from the day index 


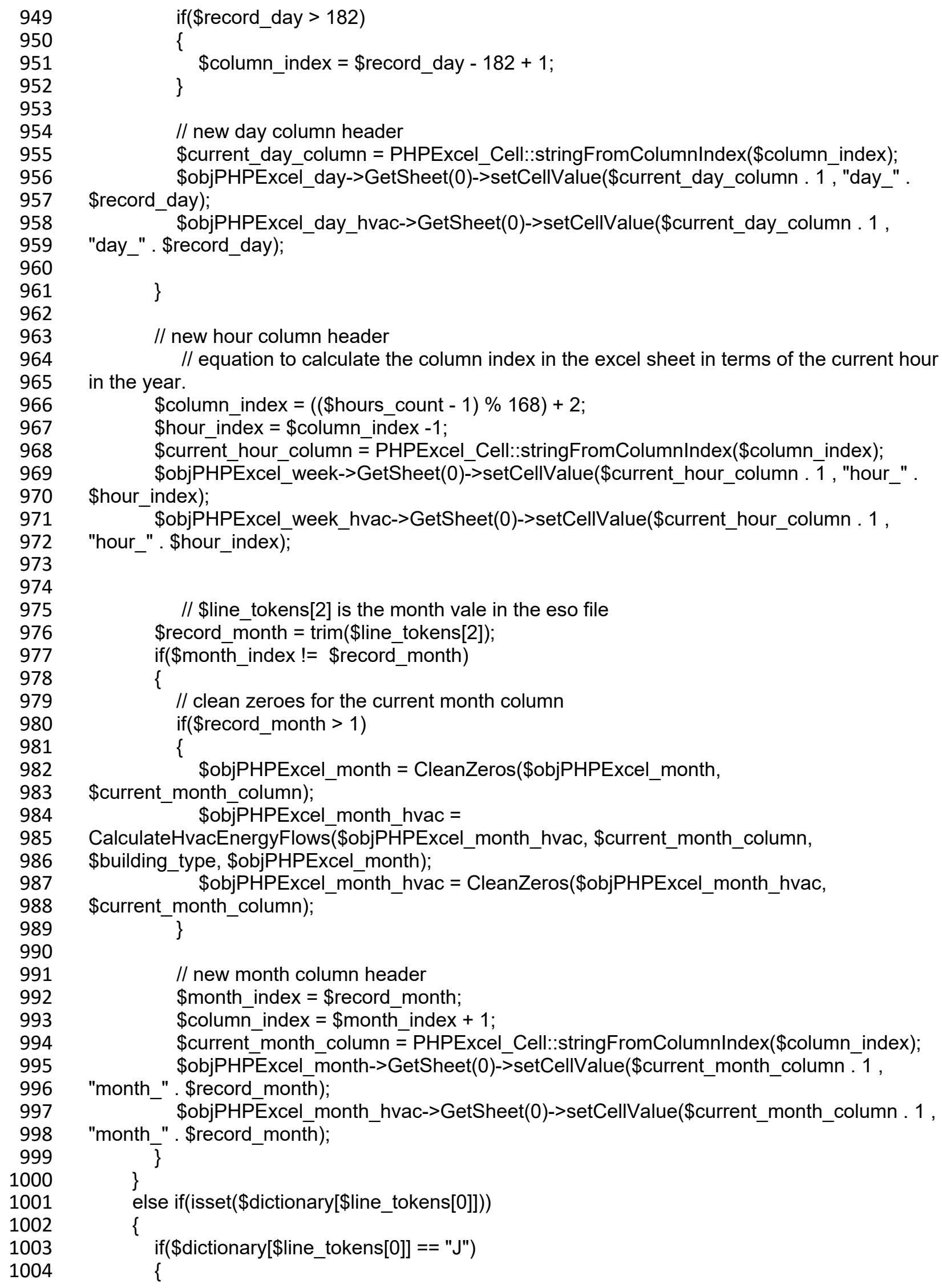


1005

1006

1007

1008

1009

1010

1011

1012

1013

1014

1015

1016

1017

1018

1019

1020

1021

1022

1023

1024

1025

1026

1027

1028

1029

1030

1031

1032

1033

1034

1035

1036

1037

1038

1039

1040

1041

1042

1043

1044

1045

1046

1047

1048

1049

1050

1051

1052

1053

1054

1055

1056

1057

1058

1059

1060
// The values is divided by 3600 to convert from $\mathrm{J}$ to $\mathrm{W}$

// \$line_tokens[0] is the ID of the physical measurement. // \$line_tokens[1] is the measured value for this phyical measurement ID. \$current_hour_values[\$line_tokens[0]] = \$line_tokens[1] / 3600.000; else

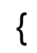

\section{\}}

\$objPHPExcel_day = CleanZeros(\$objPHPExcel_day, \$current_day_column);

\$objWriter = IPHPExcel_IOFactory::createWriter(\$objPHPExcel_day, 'CSV');

\$objWriter->save(\$diagram_dir . '/days_2.csv');

\$objWriter = \PHPExcel_IOFactory::createWriter(\$objPHPExcel_week, 'CSV');

\$objWriter->save(\$diagram_dir . '/week_'. \$week_index . '.csv');

\$objPHPExcel_month = CleanZeros(\$objPHPExcel_month, \$current_month_column);

\$objWriter = IPHPExcel_IOFactory::createWriter(\$objPHPExcel_month, 'CSV̄');

\$objWriter->save(\$diagram_dir. . '/months.csv');

\$objPHPExcel_day_hvac $=$ CalculateHvacEnergyFlows $($ \$objPHPExcel_day_hvac,

\$current_day_column, \$building_type, \$objPHPExcel_day);

\$objPHPExcel_day_hvac = CleanZeros(\$objPHPExcel_day_hvac, \$current_day_column);

\$objWriter = IPHPExcel_IOFactory::createWriter(\$objPHPExcel_day_hvac, 'CSV');

\$objWriter->save(\$diagram_dir . '/hvac_days_2.csv');

\$objWriter = IPHPExcel_IOFFactory::createWriter(\$objPHPExcel_week_hvac, 'CSV');

\$objWriter->save(\$diagram_dir . '/hvac_week_'. \$week_index .'.csv');

\$objPHPExcel_month_hvac $=$ CalculateHvacEnergyFlows $($ \$objPHPExcel_month_hvac, \$current_month_column, \$building_type, \$objPHPExcel_month);

\$objPHPExcel_month_hvac $=$ CleanZeros $($ \$objPHPExcel_month_hvac,

\$current_month_column);

\$objWriter = (PHPExcel_IOFactory::createWriter(\$objPHPExcel_month_hvac, 'CSV');

\$objWriter->save(\$diagram_dir . '/hvac_months.csv'); \}

fclose (\$myfile);

// Internal function to evaluate some records in the CSV files based on equations.

function EvaluateEquations(IPHPExcel \$objPHPExcel, \$current_column, \$building_type)

\{

\$column_array = array () ;

\$column_array[0] = 0;

\$column_array[1] = 0;

\$highest_row $=$ \$objPHPExcel->getActiveSheet()->getHighestRow();

for $($ \$row $=2 ;$ \$row $<=$ \$highest_row; \$row ++$)$

\{

\$cell_val $=$ \$objPHPExcel->getActiveSheet()->getCell(\$current_column . \$row)->getValue();

\}

\$column_array[\$row] $=\left(\right.$ empty $\left.\left(\$ c e l l \_v a l\right)\right) ~ ? 0$ : \$cell_val;

if(\$building_type == Enumerations::SMALL_OFFICE)

\{

Boiler energy transfer 
1061

1062

1063

1064

1065

1066

1067

1068

1069

1070

1071

1072

1073

1074

1075

1076

1077

1078

1079

1080

1081

1082

1083

1084

1085

1086

1087

1088

1089

1090

1091

1092

1093

1094

1095

1096

1097

1098

1099

1100

1101

1102

1103

1104

1105

1106

1107

1108

1109

1110

1111

1112

1113

1114

1115

1116 \$column_array[3] = \$column_array[2] - \$column_array[4];

// Internal heat gain from lighting = Lighting energy consumption

\$column_array[20] = \$column_array[16];

// Internal heat gain from equipment = Equipment energy consumption

\$column_array[21] = \$column_array[17];

// Energy extracted by cooling coils to DX unit = Energy extracted by cooling coils

\$column_array[61] = \$column_array[55];

$/ /$ Transmitted solar radiation $=$ Transmitted solar radiation from $($ North + South +

East + West) windows

\$column_array[37] = \$column_array[29] + \$column_array[30] + \$column_array[31] + \$column_array[32];

windows

$/ /$ Heat gain from windows $=$ Heat gain from $($ North + South + East + West $)$

\$column_array[38] = \$column_array[33] + \$column_array[34] + \$column_array[35] + \$column_array[36];

windows

$/ /$ Total window heat gains $=$ Transmitted solar radiation + Heat gain from

\$column_array[39] = \$column_array[37] + \$column_array[38];

windows

$/ /$ Heat loss from windows $=$ Heat loss from $($ North + South + East + West $)$

\$column_array[48] = \$column_array[44] + \$column_array[45] + \$column_array[46] + \$column_array[47];

// Heat loss from hot water (HW) loop pump to the surroundings = Electricity

consumption by HW pump - HW Pump frictional losses

\$column_array[8] = \$column_array[6] - \$column_array[7];

// Heat added by $\bar{H} W$ pump to the surroundings = Heat loss from hot water $(\mathrm{HW})$

pump to the surroundings

\$column_array[24] = \$column_array[8];

// Heat added by the HW pump to the fluid = HW Pump frictional losses

\$column_array[9] = \$column_array[7];

// Row 10 (Energy consumption by heating coils), Row 4 (boiler energy transfer), Row 7 (HW Pump frictional losses), Row 11 (Energy consumption by radiant panels), Row 13

(energy consumption by humidifier)

if $(\$ c$ column_array[10] > (\$column_array[4] + \$column_array[7] $))$

\{

\$column_array[10] = \$column_array[4] + \$column_array[7];

\}

\$column_array[11] = \$column_array[4] + \$column_array[7] - \$column_array[10] \$column_array[13];

if $\left(\$ c o l u m n \_a r r a y[11]<0\right)$

\{

\$column_array[11] = 0;

\}

\$column_array[10] $=$ \$column_array[4] + \$column_array[7];

// Fans electric energy consumption

\$fans_electricity $=\$$ column_array[12];

// Fans electric energy consumption for heating = Total fans electric energy

consumption * (Energy consumption by heating coils/Energy consumption by heating coils +

Energy extracted by cooling coils)

\$column_array[12] = (\$column_array[10] + \$column_array[55] !=0 $)$ ? \$fans_electricity * (\$column_array[10] / (\$column_array[10] + \$column_array[55])) : 0;

// Fans electric energy consumption for cooling $=$ Total fans electric energy consumption * (Energy extracted by cooling coils/Energy consumption by heating coils + Energy extracted by cooling coils) 
1117

1118

1119

1120

1121

1122

1123

1124

1125

1126

1127

1128

1129

1130

1131

1132

1133

1134

1135

1136

1137

1138

1139

1140

1141

1142

1143

1144

1145

1146

1147

1148

1149

1150

1151

1152

1153

1154

1155

1156

1157

1158

1159

1160

1161

1162

1163

1164

1165

1166

1167

1168

1169

1170

1171

1172
$\$$ column_array[56] $=(\$$ column_array $[10]+\$$ column_array[55] $!=0) ?$

\$fans_electricity * (\$column_array[55] / (\$column_array[10] + \$column_array[55])) : 0;

// The amount of heat added by heating coils to PSZ = Energy consumption by heating coils \$column_array[14] = \$column_array[10];

// Heat gain from building envelope $=$ Heat gain from (Walls + Roofs + Floors $)$

\$column_array[43] = \$column_array[40] + \$column_array[41] + \$column_array[42];

// Heat loss from building envelope $=$ Heat loss from (Walls + Roofs + Floors)

$\$$ column_array[52] = \$column_array[49] + \$column_array[50] $+\$$ column_array[51];

// Internal heat gain from people $=$ People sensible heat gain

\$column_array[22] = \$column_array[18];

// Internal latent gain from people = People latent energy gain

\$column_array[23] = \$column_array[19];

// Total internal gain = Lighting energy consumption + Equipment energy

consumption + People sensible heat gain + People latent energy gain

\$column_array[27] = \$column_array[20] + \$column_array[21] + \$column_array[22] + \$column_array[23];

// Heat gain from radiant panels = Energy consumption by radiant panels

\$column_array[26] = \$column_array[11];

// Row 55 (Heat extracted by cooling coils), Row 15 (heat gain from outdoor),

Row 58 (Free cooling), Row 57 (Outdoor air loss)

if $(\$$ column_array $[55]>0)$

\{

if $\left(\$ c o l u m n \_a r r a y[15]<0\right)\{$

\$column_array[15] = 0; \$column_array[58] = abs $\left(\$ c o l u m n \_a r r a y[15]\right) ;$ \} else \{

\}

\$column_array[58] = 0;

\}

else (// Heat extracted by cooling coils $=0)$

\{

if $(\$$ column_array[15] $<0)\{$

$\$$ column_array[15] = 0;

\} else \{

\$column_array[57] = 0;

\}

\}

// Energy supplied by PSZ = Fans electric energy consumption for heating +

Energy consumption by heating coils + heat gain from outdoor + energy consumption by

humidifier

\$column_array[25] = \$column_array[12] + \$column_array[10] + \$column_array[15] + \$column_array[13];

Gain Rate)

// Row 62 (Surface Heat Storage Loss Rate), Row 63 (Surface Heat Storage

if (\$column_array[62] > \$column_array[63]) \{

\$column_array[62] = \$column_array[62] - \$column_array[63];

\$column_array[63] = 0;

\} else \{

\$column_array[63] = \$column_array[63] - \$column_array[62];

\} 
1173

1174

1175

1176

1177

1178

1179

1180

1181

1182

1183

1184

1185

1186

1187

1188

1189

1190

1191

1192

1193

1194

1195

1196

1197

1198

1199

1200

1201

1202

1203

1204

1205

1206

1207

1208

1209

1210

1211

1212

1213

1214

1215

1216

1217

1218

1219

1220

1221

1222

1223

1224

1225

1226

1227

1228
// Energy extracted by PSZ = Exhaust air + Energy extracted by cooling coils + Outdoor air loss + Free cooling - Fans electric energy consumption for cooling \$column_array[59] = \$column_array[54] + \$column_array[55] +

\$column_array[57] + \$column_array[58] - \$column_array[56];

// Energy Balance

// Energy in = Energy added by HW pump to the surroundings + Energy supplied by PSZ + Total internal gains + Infiltration heat gain + Total window heat gains + Heat gain from building envelope + Surface Heat Storage Loss Rate

\$energy_in = \$column_array[24] + \$column_array[25] + \$column_array[26] +

\$column_array[27] + \$column_array[28] + \$column_array[39] + \$column_array[43] +

\$column_array[62];

$/ /$ Energy out $=$ Heat loss from windows + Heat loss from building envelope + Infiltration heat loss + Energy extracted by PSZ + Surface Heat Storage Gain Rate

\$energy_out $=$ \$column_array[48] + \$column_array[52] + \$column_array[53] +

\$column_array[59] + \$column_array[63];

\$diff = abs(\$energy_in - \$energy_out);

if(\$energy_out > \$energy_in)

\{

// Energy stored in radiant panels = energy out - energy in

\} \$column_array[64] = \$diff;

else if (\$energy_out $<$ \$energy_in)

\{

// Exhaust air $=$ Exhaust air + diff \$column_array[54] = \$column_array[54] + \$diff; // Recalculate Energy extracted by PSZ

\$column_array[59] = \$column_array[54] + \$column_array[55] + \$column_array[57] +

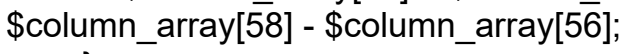

\}

// Row 54 (Exhaust air), Row 55 (Energy extracted by cooling coils)

if $(\$$ column_array $[54]<0)$

\{

\$column_array[54] =0;

\$column_array[55] = \$column_array[55] - \$diff;

// Recalculate Energy extracted by cooling coils to DX unit

\}

\$column_array[61] = \$column_array[55];

// Recalculate Energy extracted by PSZ

\$column_array[59] = \$column_array[54] + \$column_array[55] + \$column_array[57] + \$column_array[58] - \$column_array[56];

\}

else //(i.e Large and medium office)

\{

// Heat loss due to inefficiency of the boiler = Boiler natural gas consumption - Boiler energy transfer

\$column_array[3] = \$column_array[2] - \$column_array[4];

// Internal heat gain from lighting = Lighting energy consumption

\$column_array[21] = \$column_array[17];

// Internal heat gain from equipment = Equipment energy consumption

\$column_array[22] = \$column_array[18]; 
1229

1230

1231

1232

1233

1234

1235

1236

1237

1238

1239

1240

1241

1242

1243

1244

1245

1246

1247

1248

1249

1250

1251

1252

1253

1254

1255

1256

1257

1258

1259

1260

1261

1262

1263

1264

1265

1266

1267

1268

1269

1270

1271

1272

1273

1274

1275

1276

1277

1278

1279

1280

1281

1282

1283

1284

coils

// Energy extracted by cooling coils to AHU unit = Energy extracted by cooling

\$column_array[73] = \$column_array[56];

East + West) windows

// Transmitted solar radiation $=$ Transmitted solar radiation from $($ North + South +

$\$$ column_array[38] = \$column_array[30] + \$column_array[31] + \$column_array[32] +

\$column_array[33];

windows

$/ /$ Heat gain from windows $=$ Heat gain from $($ North + South + East + West $)$

\$column_array[39] = \$column_array[34] + \$column_array[35] + \$column_array[36] + \$column_array[37];

$/ /$ Total window heat gains $=$ Transmitted solar radiation + Heat gain from

windows

\$column_array[40] = \$column_array[38] + \$column_array[39];

windows

$/ /$ Heat loss from windows $=$ Heat loss from $($ North + South + East + West $)$

\$column_array[49] $=\$$ column_array[45] $+\$$ column_array[46] $+\$$ column_array[47] + \$column_array[48];

// Heat loss from hot water (HW) loop pump to the surroundings = Electricity

consumption by HW pump - HW Pump frictional losses

\$column_array[8] = \$column_array[6] - \$column_array[7];

$/ /$ Heat added by $\bar{H} W$ pump to the surroundings = Heat loss from hot water $(\mathrm{HW})$

pump to the surroundings

\$column_array[25] = \$column_array[8];

// Heat added by the HW pump to the fluid = HW Pump frictional losses

\$column_array[9] = \$column_array[7];

// Heat added by condensing water (CDW) pump to the surroundings = Electricity

consumption by CDW pump - CDW Pump frictional losses

\$column_array[65] = \$column_array[61] - \$column_array[62];

the surroundings

// Heat added by $\overline{C D W}$ pump to the surroundings $=$ Heat loss from CDW pump to

\$column_array[67] = \$column_array[65];

// Heat added by the CDW pump to the fluid $=$ CDW Pump frictional losses

\$column_array[69] = \$column_array[62];

// Heat added by chilled water (CHW) pump to the surroundings = Electricity

consumption by CHW pump - CHW Pump frictional losses

\$column_array[66] = \$column_array[63] - \$column_array[64];

the surroundings

$/ /$ Heat added by $\mathrm{CHW}$ pump to the surroundings = Heat loss from $\mathrm{CHW}$ pump to

\$column_array[68] = \$column_array[66];

// Heat added by the CHW pump to the fluid $=\mathrm{CHW}$ Pump frictional losses

\$column_array[70] = \$column_array[64];

// Energy extracted from chillers to cooling towers = Heat added by the $\mathrm{CHW}$

pump to the fluid + electric energy consumption by chillers + Heat extracted by cooling coils

\$column_array[74] = \$column_array[70] + \$column_array[71] + \$column_array[73]; losses)

// if Energy consumption by heating coils > (boiler energy transfer + HW Pump frictional

$$
\begin{aligned}
& \text { if }(\$ \text { column_array[10] > (\$column_array[4] }+ \text { \$column_array[7] })) \\
& \{\text { // Energy consumption by heating coils = boiler energy transfer + HW Pump frictional } \\
& \text { \$column_array[10] = \$column_array[4] + \$column_array[7]; }
\end{aligned}
$$

// Energy consumption by radiant panels = (boiler energy transfer + HW Pump frictional 
1285

1286

1287

1288

1289

1290

1291

1292

1293

1294

1295

1296

1297

1298

1299

1300

1301

1302

1303

1304

1305

1306

1307

1308

1309

1310

1311

1312

1313

1314

1315

1316

1317

1318

1319

1320

1321

1322

1323

1324

1325

1326

1327

1328

1329

1330

1331

1332

1333

1334

1335

1336

1337

1338

1339

1340 losses - energy consumption by heating coils - energy consumption by VAV-Reheat coils)

\$column_array[12] = \$column_array[4] + \$column_array[7] - \$column_array[10] \$column_array[11] - \$column_array[14];

// If Energy consumption by radiant panels $<0$

if $(\$$ column_array[12] $<0)\{$

\$column_array[12] = 0 ;

// VAV-Reheat energy consumption = boiler energy transfer + HW Pump

frictional losses - Energy consumption by radiant panels

\}

\$column_array[11] = \$column_array[4] + \$column_array[7] - \$column_array[10];

// row 13,57 ( Fans: electricity and perform 2 equations)

// Fans electric energy consumption

\$fans_electricity = \$column_array[13];

// Fans electric energy consumption for heating = Total fans electric energy

consumption * (Energy consumption by heating coils/Energy consumption by heating coils +

Energy extracted by cooling coils)

\$column_array[13] = $(\$$ column_array[10] + \$column_array[56] != 0) ? \$fans_electricity * (\$column_array[10] / (\$column_array[10] + \$column_array[56])) : 0;

// Fans electric energy consumption for cooling = Total fans electric energy consumption * (Energy extracted by cooling coils/Energy consumption by heating coils + Energy extracted by cooling coils)

\$column_array[57] $=(\$$ column_array $[10]+\$$ column_array[56] $!=0) ?$

\$fans_electricity * (\$column_array[56] / (\$column_array[10] + \$column_array[56])) : 0;

// The amount of heat added by heating coils to AHU = Energy consumption by

heating coils

\$column_array[15] = \$column_array[10];

$/ /$ Heat gain from building envelope $=$ Heat gain from (Walls + Roofs + Floors $)$

\$column_array[44] = \$column_array[41] + \$column_array[42] + \$column_array[43];

$/ /$ Heat loss from building envelope $=$ Heat loss from (Walls + Roofs + Floors)

\$column_array[53] = \$column_array[50] + \$column_array[51] + \$column_array[52];

$/ /$ Internal heat gain from people $=$ People sensible heat gain

\$column_array[23] = \$column_array[19];

// Internal latent gain from people = People latent energy gain

\$column_array[24] = \$column_array[20];

// Total internal gain = Lighting energy consumption + Equipment energy

consumption + People sensible heat gain + People latent energy gain

\$column_array[28] = \$column_array[21] + \$column_array[22] + \$column_array[23] + \$column_array[24];

// Heat gain from radiant panels = Energy consumption by radiant panels

\$column_array[27] = \$column_array[12];

// row 16 (Heat gain from outdoor) and row 58 (Outdoor air loss)

if $(\$$ column_array[16] $<0)\{$

\$column_array[58] = abs $(\$$ column_array[16]);

\$column_array[16] = 0;

\} else \{

\}

\$column_array[58] = 0;

// Energy supplied by AHU = Fans electric energy consumption for heating + energy consumption by humidifier + Energy consumption by heating coils + heat gain from outdoor \$column_array[26] = \$column_array[13] + \$column_array[14] +

\$column_array[15] + \$column_array[16];

// Heat added by VAV-Reheat $=$ VAV-Reheat energy consumption 


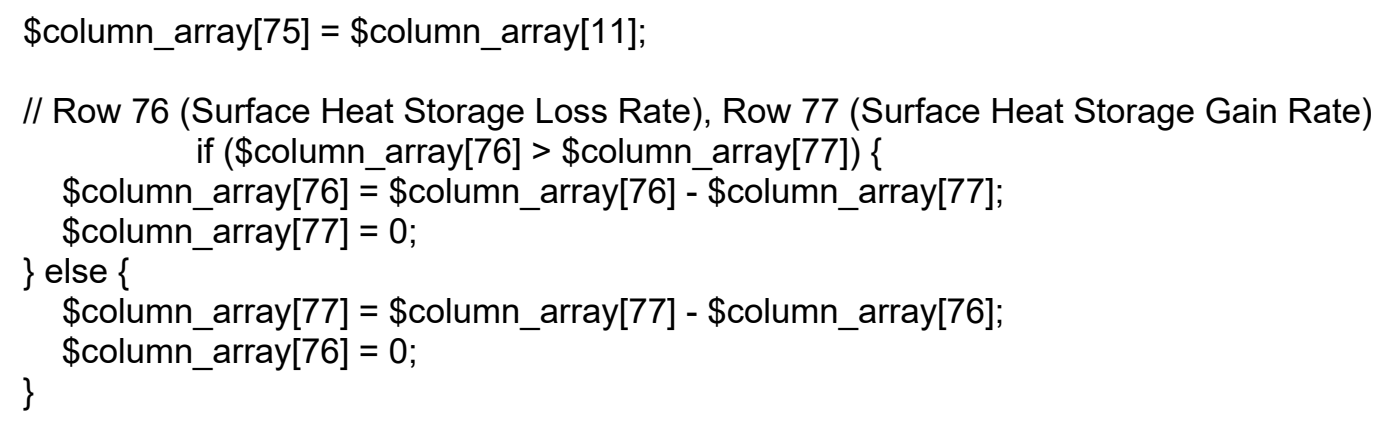

// Energy extracted by AHU = Exhaust air + Energy extracted by cooling coils + Outdoor air loss + Free cooling - Fans electric energy consumption for cooling \$column_array[60] $=(\$$ column_array[55] + \$column_array[56] + \$column_array[58] + \$column_array[59] - \$column_array[57]);

\section{// Adjust Energy Balance, Row 78 (Energy stored in radiant panels)}

\$column_array[78] = 0;

// Energy in = Energy added by HW pump to the surroundings + Energy supplied by $\mathrm{AHU}+$ Energy consumption by radiant panel + Total internal gains + Infiltration heat gain + Total window heat gains + Heat gain from building envelope + Energy added by CDW pump to the surroundings + Energy added by CHW pump to the surroundings + VAV-Rehheat coils energy consumption + Surface Heat Storage Loss Rate

\$energy_in = \$column_array[25] + \$column_array[26] + \$column_array[27] + \$column_array[28] + \$column_array[29] + \$column_array[40] + \$column_array[44] + \$column_array[67] + \$column_array[68] + \$column_array[75] + \$column_array[76];

$/ /$ Energy out $=$ Heat loss from windows + Heat loss from building envelope + Infiltration heat loss + Energy extracted by AHU + Surface Heat Storage Gain Rate

\$energy_out $=$ \$column_array[49] + \$column_array[53] + \$column_array[54] + \$column_array[60] + \$column_array[77];

\$diff = abs $($ Senergy_in - \$energy_out); if $(\$ \operatorname{diff}>10)$

\{

// Row 56 (Enegry extracted by cooling coils), Row 55 (Exhaust air), Row 59 (Free cooling), Row 78 (Energy stored in radiant panels)

\{ if $(\$$ column_array $[56]==0)$

if(\$energy_out > \$energy_in)

\{

\$column_array[78] = \$diff; \} 


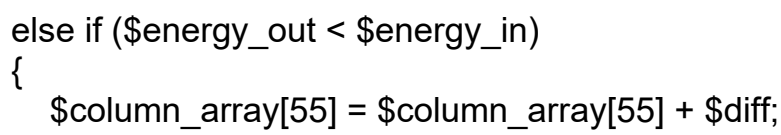

// Recalculate Energy extracted by AHU = Exhaust air + Energy extracted by cooling coils + Outdoor air loss + Free cooling - Fans electric energy consumption for cooling

\$column_array[60] $=(\$$ column_array[55] $+\$$ column_array[56] + \$column_array[58] + \$column_array[59] - \$column_array[57]); \}

\} else if (\$column_array[56] > 0) \{

if(\$energy_in > \$energy_out) \{

// Recalculate Energy extracted by AHU = Exhaust air + Energy extracted by cooling coils + Outdoor air loss + Free cooling - Fans electric energy consumption for cooling

\$column_array[60] $=(\$$ column_array[55] + \$column_array[56] + \$column_array[58] + \$column_array[59]-\$column_array[57]);

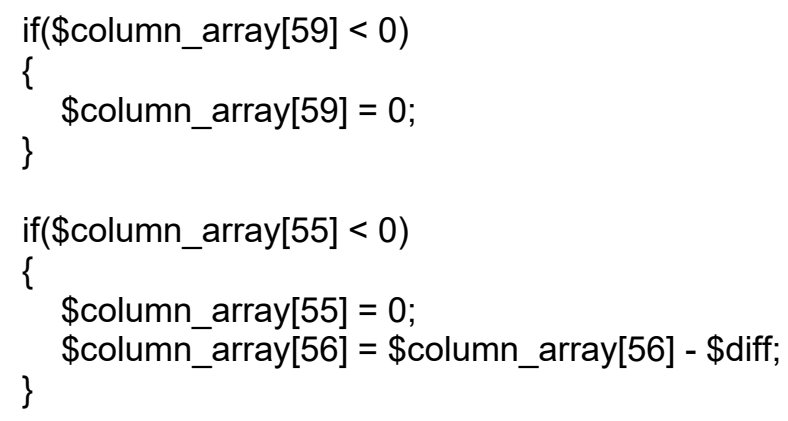

// Recalculate Energy extracted by AHU

\$column_array[60] $=(\$$ column_array[55] + \$column_array[56] + \$column_array[58] + \$column_array[59] - \$column_array[57]);

cooling coils)

// Row 73 (Energy extracted from cooling coils to chillers = Energy extracted by

\$column_array[73] = \$column_array[56];

// Energy extracted from chillers to cooling towers = Heat added by the CHW pump to the fluid + Electric energy consumption by chillers + Energy extracted by cooling coils \} \$column_array[74] = \$column_array[70] + \$column_array[71] + \$column_array[73];

// begin from row 2 in the csv for $($ \$row $=2$; \$row $<=$ \$highest_row; \$row++) 


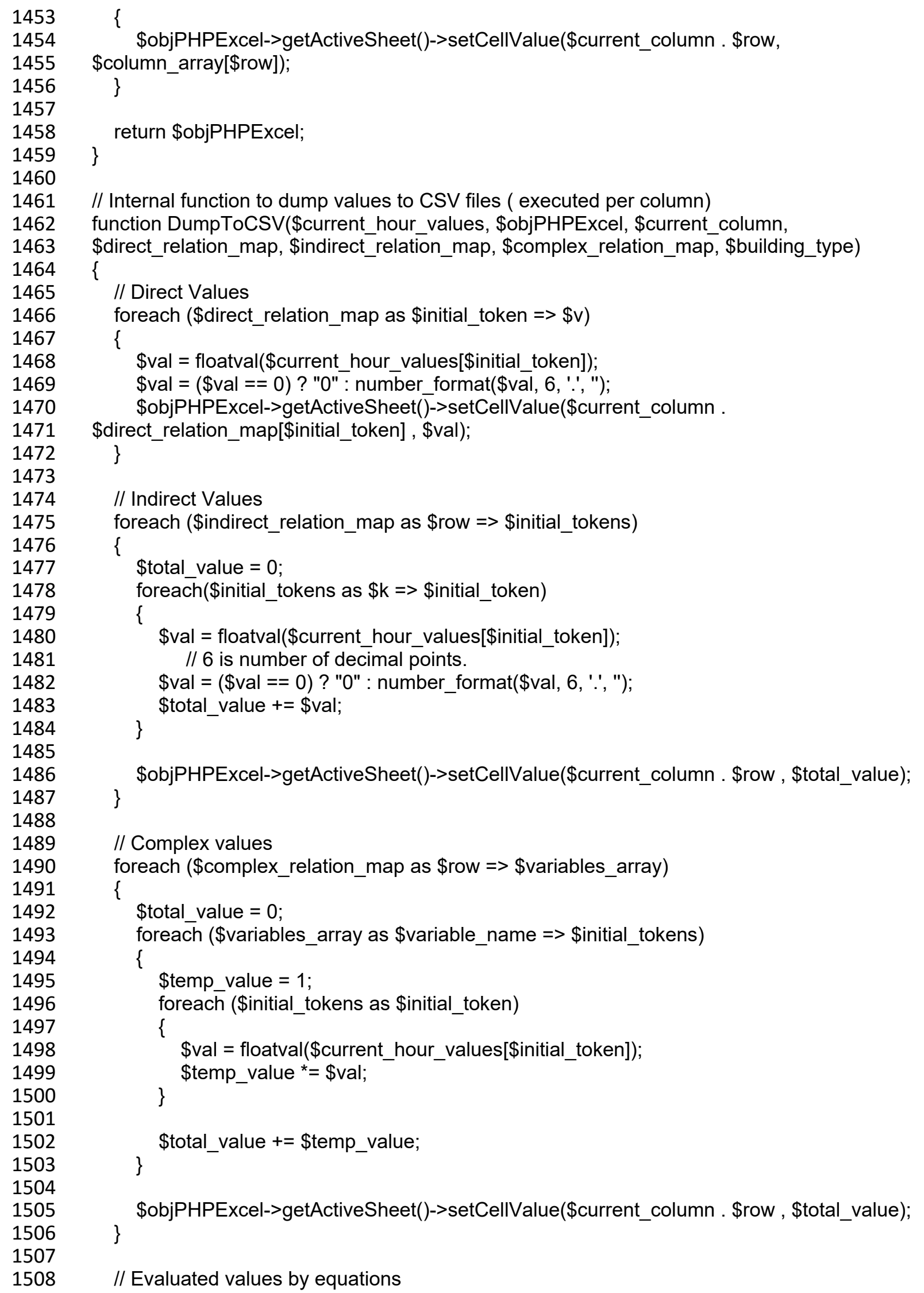


\$objPHPExcel = EvaluateEquations(\$objPHPExcel, \$current_column, \$building_type);

$$
\text { return \$objPHPExcel; }
$$


1565

1566

1567

1568

1569

1570

1571

1572

1573

1574

1575

1576

1577

1578

1579

1580

1581

1582

1583

1584

1585

1586

1587

1588

1589

1590

1591

1592

1593

1594

1595

1596

1597

1598

1599

1600

1601

1602

1603

1604

1605

1606

1607

1608

1609

1610

1611

1612

1613

1614

1615

1616

1617

1618

1619

1620

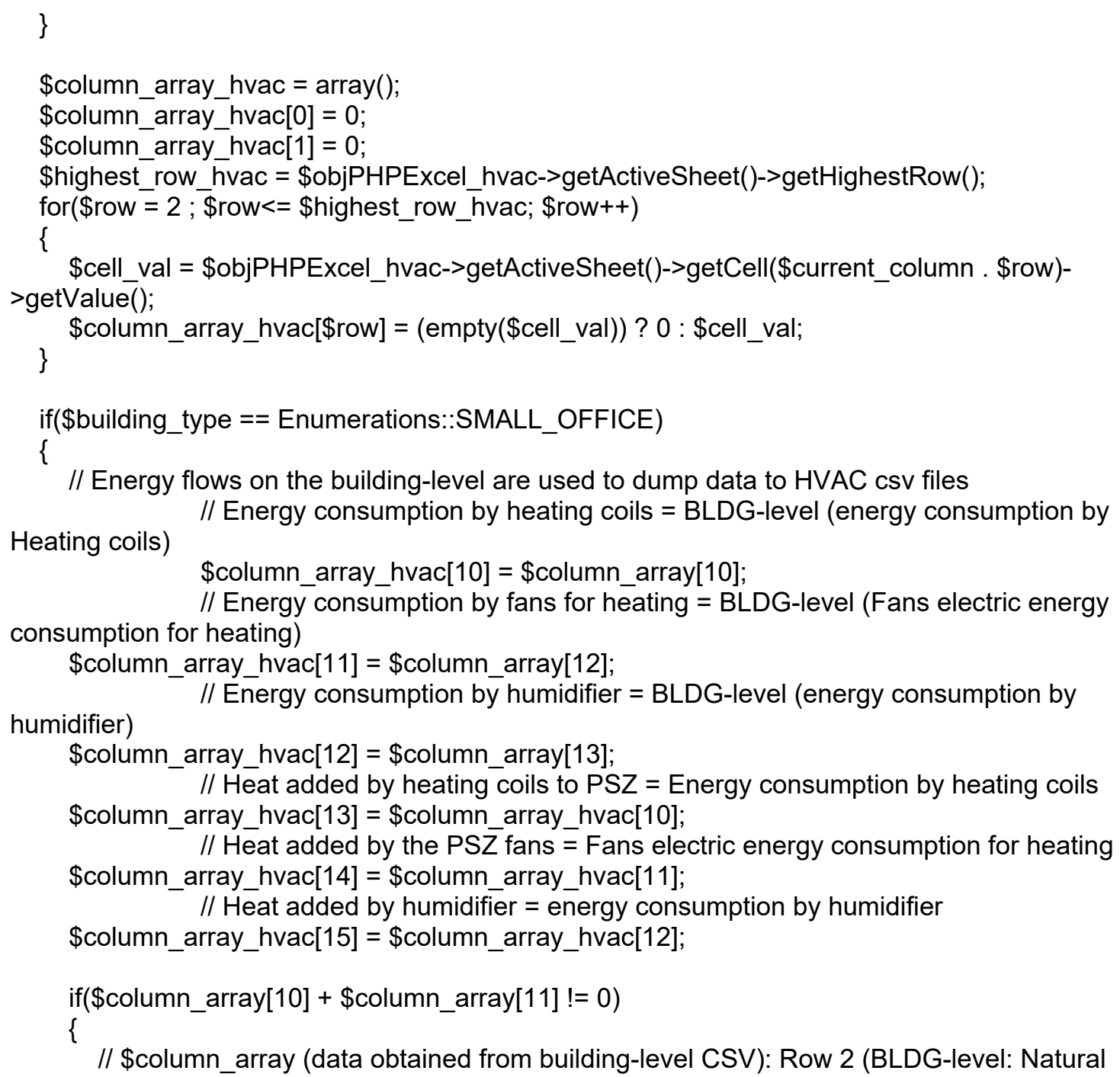


1621

1622

1623

1624

1625

1626

1627

1628

1629

1630

1631

1632

1633

1634

1635

1636

1637

1638

1639

1640

1641

1642

1643

1644

1645

1646

1647

1648

1649

1650

1651

1652

1653

1654

1655

1656

1657

1658

1659

1660

1661

1662

1663

1664

1665

1666

1667

1668

1669

1670

1671

1672

1673

1674

1675

1676

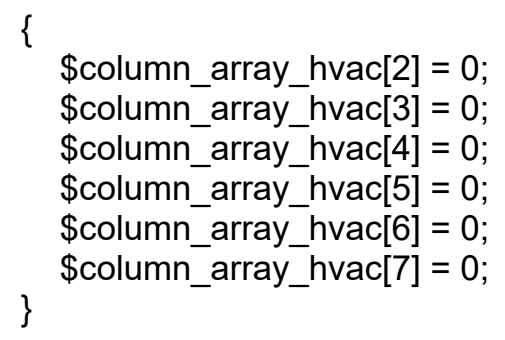

// \$column_array (data obtained from building-level CSV): Row 15 (heat gain from outdoor), Row 55 (energy exctracted by cooling coils), Row 56 (Fans electric energy consumption for cooling), Row 57 (outdoor air loss), Row 58 (free cooling)

// \$column_array_hvac: Row 6 (Electric energy consumption by HW pump), Row 7 (HW frictional losses), Row 8 (HW pump heat loss to surroundings), Row 9 (HW frictional losses), Row 20 (heat gain from outdoor), Row 21 (Outdoor air loss), Row 24 (energy extracted by cooling coils), Row 25 (Free cooling), Row 26 (Fans electric energy consumption for cooling)

\$column_array_hvac[8] = \$column_array_hvac[6] - \$column_array_hvac[7];

\$column_array_hvac[9] = \$column_array_hvac[7];

\$column_array_hvac[20] = \$column_array[15];

\$column_array_hvac[21] = \$column_array[57];

\$column_array_hvac[25] = \$column_array[58];

\$column_array_hvac[26] = \$column_array[56];

\$column_array_hvac[24] = \$column_array[55] - \$column_array_hvac[26];

if( $\$$ column_array_hvac[24] != 0)

\{

// \$column_array (data obtained from building-level CSV): Row 54 (Exhaust air)

// \$column_array_hvac: Row 16 (Return air from plenum), Row 17

(Exhaust air), Row 24 (energy extracted by cooling coils), Row 27 (Fans electric energy

consumption for cooling), Row 29 (Energy extracted by cooling coils)

\} \$column_array_hvac[16] $=0$

\$column_array_hvac[29] = \$column_array_hvac[24] + \$column_array_hvac[27];

\$column_array_hvac[17] $=(\$$ column_array_hvac[24] == 0) ? \$column_array[54] : 0;

if(\$column_array_hvac[17] > \$column_array_hvac[16])

\{

\$column_array_hvac[16] = \$column_array_hvac[17];

\}

// \$column_array (data obtained from building-level CSV): Row 60 (Electric energy consumption by $D X$ unit)

// \$column_array_hvac: Row 13 (Energy consumption by heating coils), Row 14

(Fans electric energy consumption for heating), Row 15 (Heat added by humidifier), Row 16

(Return air), Row 17 (Exhaust air), Row 18 (Recirculated air), Row 19 (Heat added by

recirculated air to the supply air), Row 20 (heat gain from outdoor), Row 21 (Outdoor air loss),

Row 22 (Heat supplied to zones), Row 23 (Heat gain from building), Row 26 (Fans electric

energy consumption for cooling), Row 27 (Fans electric energy consumption for cooling), Row 28

(Electric energy consumption by DX unit)

\$column_array_hvac[18] = \$column_array_hvac[16] - \$column_array_hvac[17];

\$column_array_hvac[19] = \$column_array_hvac[18];

\$temp_val $=\$$ column_array_hvac[13] $+\$$ column_array_hvac[14] + \$column_array_hvac[15]

+ \$column_array_hvac[19] + \$column_array_hvac[20] - \$column_array_hvac[21];

\$column_array_hvac[22] $=($ \$temp_val $>$ \$column_array_hvac[16] $)$ ?

\$column_array_hvac[16]:0;

\$column_array_hvac[23] $=(\$$ temp_val $<$ \$column_array_hvac[16] $) ?$

\$column_array_hvac[16] - \$temp_val : 0; 
\$column_array_hvac[27] = \$column_array_hvac[26];

\$column_array_hvac[28] $=$ \$column_array[60];

// \$column_array (data obtained from building-level CSV): Row 10 (Heating coil energy consumption), Row 13 (energy consumption by humidifier), Row 54 (Exhaust air), Row 55 (Energy extracted by cooling coil), Row 58 (Free cooling)

// \$column_array_hvac: Row 13 (Energy consumption by heating coils), Row 14 (Fans electric energy consumption), Row 15 (Heat added by humidifier), Row 16 (Return air), Row 17 (Exhaust air), Row 18 (Recirculated air), Row 19 (Heat added by recirculated air to the supply air), Row 20 (heat gain from outdoor), Row 21 (Outdoor air loss), Row 22 (Heat supplied to zones), Row 23 (Heat gain from building), Row 24 (energy extracted by cooling coils), Row 25 (free cooling), Row 26 (Fans electric energy consumption), Row 30 (Heat supplied to zones), Row 31 (return air to plenum), Row 32 (Exhaust air), Row 33 (Energy added by heating coils to cooling coils), Row 34 (Fans electric energy consumption), Row 35 (heat added by humidifier to cooling coils)

\{

$$
\text { if }\left(\left(\$ c o l u m n \_a r r a y[10]>\right.\right.\text { column_array[55])) }
$$

\$column_array_hvac[32] = 0;

\$column_array_hvac[30] = \$column_array_hvac[13] +\$column_array_hvac[14] + \$column_array_hvac[15] + \$column_array_hvac[19] + \$column_array_hvac[20] -

\$column_array_hvac[21] - \$column_array_hvac[24] - \$column_array_hvac[25];

if(\$column_array_hvac[30] $<$ \$column_array_hvac[16])

\{

\$column_array_hvac[23] = \$column_array_hvac[16] - \$column_array_hvac[30];

\} else if (\$column_array_hvac[30] > \$column_array_hvac[16])

\{ 
1733

1734

1735

1736

1737

1738

1739

1740

1741

1742

1743

1744

1745

1746

1747

1748

1749

1750

1751

1752

1753

1754

1755

1756

1757

1758

1759

1760

1761

1762

1763

1764

1765

1766

1767

1768

1769

1770

1771

1772

1773

1774

1775

1776

1777

1778

1779

1780

1781

1782

1783

1784

1785

1786

1787

1788 \$column_array_hvac[33] - \$column_array_hvac[34] - \$column_array_hvac[35] ; \}

\$column_array_hvac[31] = \$column_array_hvac[30];

\$column_array_hvac[29] = \$column_array_hvac[24] + \$column_array_hvac[27] + \$column_array_hvac[33] + \$column_array_hvac[34] + \$column_array_hvac[35];

\} else (//i.e. Large and medium office)

\{

// Energy flows on the building-level are used to dump data to HVAC csv files // Energy consumption by heating coils = BLDG-level (energy consumption by Heating coils)

\$column_array_hvac[10] = \$column_array[10];

// Energy consumption by fans for heating = BLDG-level (Fans electric energy consumption for heating)

\$column_array_hvac[11] = \$column_array[13];

humidifier)

// Energy consumption by humidifier = BLDG-level (energy consumption by

\$column_array_hvac[12] = \$column_array[14];

// Heat added by heating coils to AHU = Energy consumption by heating coils

\$column_array_hvac[13] = \$column_array_hvac[10];

$/ /$ Heat added by the AHU fans = Fans electric energy consumption for heating

\$column_array_hvac[14] = \$column_array_hvac[11];

// Heat added by humidifier = energy consumption by humidifier

\$column_array_hvac[15] = \$column_array_hvac[12];

if $\left(\$ c o l u m n \_a r r a y[10]+\$\right.$ column_array[11] $+\$$ column_array[12] != 0)

\{

// \$column_array (data obtained from building-level CSV): Row 2 (BLDG-level: Natural gas consumption), Row 3 (Boiler heat loss due to inefficiency), Row 4 (Boiler energy transfer), Row 5 (Electric energy consumption by boiler), Row 6 (Electric energy consumption by HW pump), Row 7 (HW frictional losses), Row 10 (BLDG-level: energy consumption by heating coil), Row 11 (energy consumption by VAV-Reheat, Row 12 (energy consumption by radiant panel) // \$column_array_hvac: Row 2 (Natural gas consumption), Row 3 (Boiler heat loss due to inefficiency), Row $\overline{4}$ (Boiler energy transfer), Row 5 (Electric energy consumption by boiler), Row 6 (Electric energy consumption by HW pump), Row 7 (HW frictional losses) \$column_array_hvac[2] = \$column_array[2] * $($ column_array[10] $/$

(\$column_array[10] $+\$$ column_array[11] $+\$$ column_array[12]));

\$column_array_hvac[3] $=\$$ column_array[3] ${ }^{-}(\$$ column_array[10] / $(\$$ column_array $[10]+$ \$column_array[11] + \$column_array[12]));

$\$$ column_array_hvac $[\overline{4}]=\$$ column_array $[4] *$ * $\$$ column_array[10] $/(\$$ column_array $[10]+$

\$column_array[11] + \$column_array[12]));

\$column_array_hvac[5] = \$column_array[5] * $($ column_array[10] / $(\$$ column_array[10] +

\$column_array[11] + \$column_array[12]));

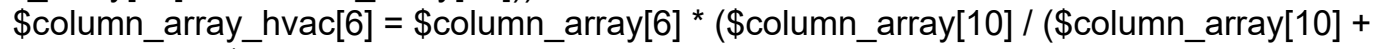
\$column_array[11] $+\$$ column_array[12]));

\$column_array_hvac[7] $=\$$ column_array[7] * $(\$$ column_array[10] $/(\$$ column_array[10] +

\$column_array[11] + \$column_array[12]));

\}

else

\{

$\$$ column_array_hvac[2] $=0$;

$\$$ column_array_hvac[3] = 0;

$\$$ column_array_hvac[4] = 0;

\$column_array_hvac[5] = 0;

\$column_array_hvac[6] = 0; 
\}

\$column_array_hvac[7] = 0;

// \$column_array (data obtained from building-level CSV): Row 16 (Heat gain from outdoor), Row 56 (energy exctracted by cooling coils), Row 57 (Fans electric energy consumption for cooling), Row 58 (outdoor air loss), Row 59 (free cooling)

// \$column_array_hvac: Row 6 (Electric energy consumption by HW pump), Row 7 (HW frictional losses), Row 8 (HW pump heat loss to surroundings), Row 9 (HW frictional losses), Row 20 (heat gain from outdoor), Row 21 (Outdoor air loss), Row 24 (energy extracted by cooling coils), Row 25 (Free cooling), Row 26 (Fans electric energy consumption for cooling), Row 27 (Fans electric energy consumption for cooling)

\$column_array_hvac[8] = \$column_array_hvac[6] - \$column_array_hvac[7];

\$column_array_hvac[9] $=$ \$column_array_hvac[7];

\$column_array_hvac[20] = \$column_array[16];

\$column_array_hvac[21] = \$column_array[58];

\$column_array_hvac[25] = \$column_array[59];

\$column_array_hvac[26] = \$column_array[57];

\$column_array_hvac[27] = \$column_array_hvac[26];

\$column_array_hvac[24] = \$column_array[56] - \$column_array_hvac[26];

if $(\$$ column_array_hvac[24] $<0)$

\{

// \$column_array (data obtained from building-level CSV): Row 55 (Exhaust air) // \$column_array_hvac: Row 16 (Return air from plenum), Row 17

(Exhaust air), Row 24 (energy extracted by cooling coils)

$$
\text { \$column_array_hvac[24] =0; }
$$

\}

\$column_array_hvac[17] = (\$column_array_hvac[24] == 0) ? \$column_array[55] : 0; if(\$column_array_hvac[17] > \$column_array_hvac[16])

\{

\$column_array_hvac[16] = \$column_array_hvac[17]; \}

// \$column_array (data obtained from building-level CSV): Row 10 (Heating coil energy consumption), Row 56 (energy extracted by cooling coils), Row Row 61 (CDW pump electric energy), Row 62 (CDW pump frictional losses), Row 63 (CHW pump electric energy), Row 64 (CHW pump frictional losses), Row 65 (CDW pump heat loss to surroundings), Row 66 (CDW pump heat loss to surroundings), Row 71 (Chiller electric energy consumption), Row 72 (Cooling tower fans electric energy consumption)

// \$column_array_hvac: Row 13 (Heating coil enegry consumption), Row 14 (Heat added by AHU fans), Row 15 (Heat added by humidifier), Row 16 (Return airto AHU), Row 18 (recirculated air), Row 19 (heat added by recirculated air), Row 20 (heat gain from outdoor), Row 21 (Outdoor air loss), Row 22 (heat supplied to zones), Row 23 (heat gain from building), Row 24 (energy extracted by cooling coils), Row 25 (free cooling), Row 28 (CDW pump electric energy), Row 29 (CDW pump fricitional losses), Row 30 (CHW pump electric energy), Row 31 (CHW pump frictional losses), Row 32 (CDW pump heat loss to surroundings), Row 33 (CHW heat loss to surroundings), Row 34 (CDW pump frictional losses to cooling towers), Row 35 (CHW pump frictional losses to chilled water loop), Row 37 (energy extracted by chilled water loop to chiller), Row 38 (chiller electric energy consumption), Row 39 (Cooling tower fans electric energy consumption), Row 40 (Cooling tower fans electric energy), Row 41 (energy exctracted by chillers to cooling towers), Row 42 (Heat supplied to zones from AHU), Row 43 (Return air to plenum), Row 44 (Exhaust air), Row 45 (Energy added by heating coil to be extracted by cooling coils), Row 46 (Heat added by fans to be extracted by cooling coils), Row 47 (heat added by humidifier to be extracted by cooling coils)

\$column_array_hvac[28] = \$column_array[61];

\$column_array_hvac[29] = \$column_array[62];

\$column_array_hvac[30] = \$column_array[63];

\$column_array_hvac[31] = \$column_array[64];

\$column_array_hvac[32] = \$column_array[65]; 


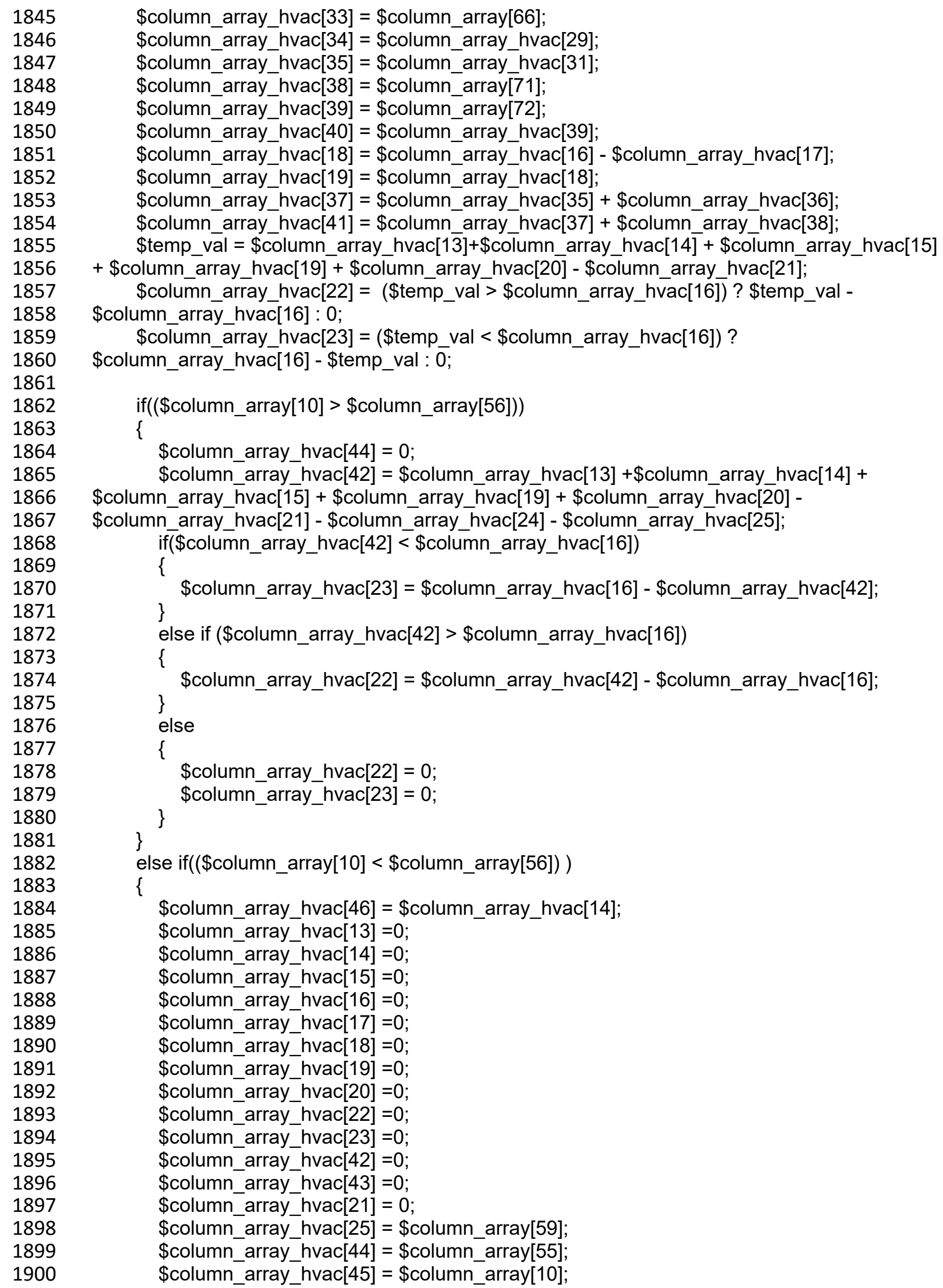


1901

1902

1903

1904

1905

1906

1907

1908

1909

1910

1911

1912

1913

1914

1915

1916

1917

1918

1919

1920

1921

1922

1923

1924

1925

1926

1927

1928

1929

1930

1931

1932

1933

1934

1935

1936

1937

1938

1939

1940

1941

1942

1943

1944

1945

1946

1947

1948

1949

1950

1951

1952

1953

1954

1955

1956

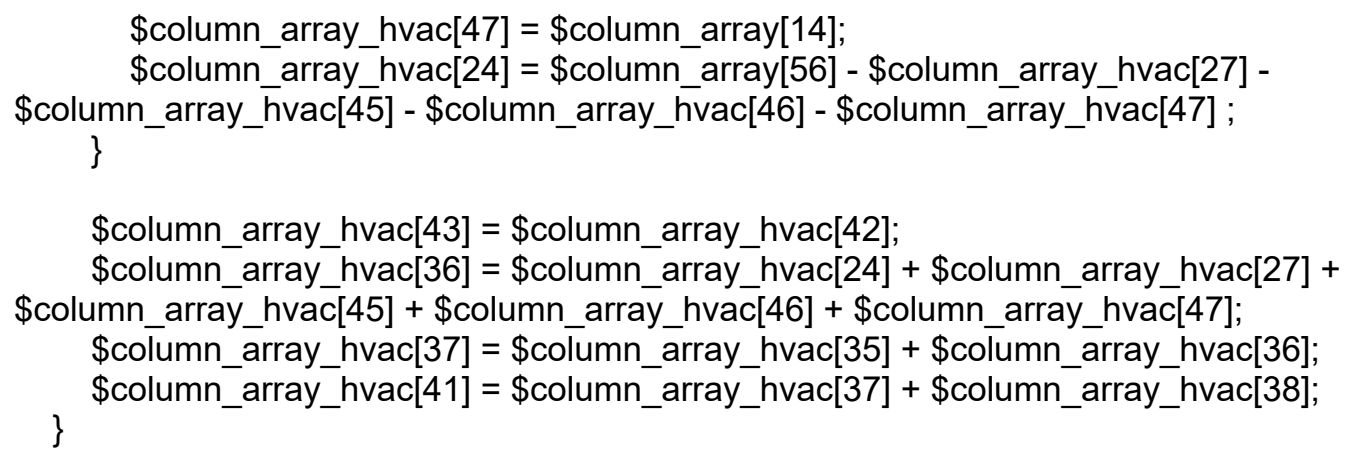

// Row 11 (Energy consumption by AHU fans for heating), Row 26 (Energy consumption by AHU fans for cooling)

//Calculate HVAC energy flows for (small, medium, and large) days and months values function CalculateHvacEnergyFlows(\$objPHPExcel_hvac, \$current_column, \$building_type, \$objPHPExcel)

\{

\$column_array_hvac $=$ array () ;

\$column_array_hvac[0] $=0$;

\$column_array_hvac[1] = 0;

\$highest_row $=$ \$objPHPExcel_hvac->getActiveSheet()->getHighestRow(); for $($ \$row $=2 ;$ Srow $<=$ \$highest_row; $\$$ row ++$)$

// \$column_array (data obtained from building-level CSV): Row 10 (Heating coil energy consumption), Row 13 (energy consumption by humidifier), Row 54 (Exhaust air), Row 55 (Energy extracted by cooling coil), Row 58 (Free cooling)

// \$column_array_hvac: Row 13 (Energy consumption by heating coils), Row 14 (Fans electric energy consumption), Row 15 (Heat added by humidifier), Row 16 (Return air), Row 17 
1957

1958

1959

1960

1961

1962

1963

1964

1965

1966

1967

1968

1969

1970

1971

1972

1973

1974

1975

1976

1977

1978

1979

1980

1981

1982

1983

1984

1985

1986

1987

1988

1989

1990

1991

1992

1993

1994

1995

1996

1997

1998

1999

2000

2001

2002

2003

2004

2005

2006

2007

2008

2009

2010

2011

2012
(Exhaust air), Row 18 (Recirculated air), Row 19 (Heat added by recirculated air to the supply air), Row 20 (heat gain from outdoor), Row 21 (Outdoor air loss), Row 22 (Heat supplied to zones), Row 23 (Heat gain from building), Row 24 (energy extracted by cooling coils), Row 25 (free cooling), Row 26 (Fans electric energy consumption), Row 30 (Heat supplied to zones), Row 31 (return air to plenum), Row 32 (Exhaust air), Row 33 (Energy added by heating coils to cooling coils), Row 34 (Fans electric energy consumption), Row 35 (heat added by humidifier to cooling coils)

\{

$$
\text { if ((\$column_array_hvac[10] > \$column_array_hvac[24])) }
$$

\$column_array_hvac[32] $=0$;

\$column_array_hvac[30] = \$column_array_hvac[13] +\$column_array_hvac[14] +

\$column_array_hvac[15] + \$column_array_hvac[19] + \$column_array_hvac[20] -

\$column_array_hvac[21] - \$column_array_hvac[24] - \$column_array_hvac[25];

if(\$column_array_hvac[30] $<$ \$column_array_hvac[16])

\{

\}

\$column_array_hvac[23] = \$column_array_hvac[16] - \$column_array_hvac[30];

else if (\$column_array_hvac[30] > \$column_array_hvac[16])

\{

\}

\$column_array_hvac[22] = \$column_array_hvac[30] - \$column_array_hvac[16];

else

\{

\$column_array_hvac[22] = 0;

\}

\$column_array_hvac[23] =0;

\}

else if $((\$$ column_array_hvac[10] $<$ \$column_array_hvac[24]) )

\{

\$column_array_hvac[34] = \$column_array_hvac[14];

\$column_array_hvac[13] =0;

$\$$ column_array_hvac[14] $=0$;

$\$$ column_array_hvac[15] $=0$;

\$column_array_hvac[16] $=0$;

$\$$ column_array_hvac[17] $=0$;

\$column_array_hvac[18] =0;

\$column_array_hvac[19] =0;

\$column_array_hvac[20] $=0$;

$\$$ column_array_hvac[22] $=0$;

$\$$ column_array_hvac[23] =0;

$\$$ column_array_hvac[30] $=0$;

\$column_array_hvac[31] =0;

\$column_array_hvac[21] = 0;

\$column_array_hvac[33] = \$column_array_hvac[10];

\$column_array_hvac[35] = \$column_array_hvac[12];

\$row55 = \$objPHPExcel->getActiveSheet()->getCell(\$current_column . "55")->getValue();

\$column_array_hvac[24] = \$row55 - \$column_array_hvac[26] - \$column_array_hvac[33] -

\$column_array_hvac[34] - \$column_array_hvac[35] ;

\}

\$column_array_hvac[31] = \$column_array_hvac[30]

\$column_array_hvac[29] = \$column_array_hvac[24] + \$column_array_hvac[27] +

\$column_array_hvac[33] + \$column_array_hvac[34] + \$column_array_hvac[35];

\}

else (// i.e. Large and medium office)

\{ 
2013

2014

2015

2016

2017

2018

2019

2020

2021

2022

2023

2024

2025

2026

2027

2028

2029

2030

2031

2032

2033

2034

2035

2036

2037

2038

2039

2040

2041

2042

2043

2044

2045

2046

2047

2048

2049

2050

2051

2052

2053

2054

2055

2056

2057

2058

2059

2060

2061

2062

2063

2064

2065

2066

2067

2068

// \$column_array (data obtained from building-level CSV): Row 10 (Heating coil energy consumption), Row 56 (energy extracted by cooling coils), Row Row 61 (CDW pump electric energy), Row 62 (CDW pump frictional losses), Row 63 (CHW pump electric energy), Row 64 (CHW pump frictional losses), Row 65 (CDW pump heat loss to surroundings), Row 66 (CDW pump heat loss to surroundings), Row 71 (Chiller electric energy consumption), Row 72 (Cooling tower fans electric energy consumption)

// \$column_array_hvac: Row 13 (Heating coil enegry consumption), Row 14 (Heat added by AHU fans), Row 15 (Heat added by humidifier), Row 16 (Return airto AHU), Row 18 (recirculated air), Row 19 (heat added by recirculated air), Row 20 (heat gain from outdoor), Row 21 (Outdoor air loss), Row 22 (heat supplied to zones), Row 23 (heat gain from building), Row 24 (energy extracted by cooling coils), Row 25 (free cooling), Row 28 (CDW pump electric energy), Row 29 (CDW pump fricitional losses), Row 30 (CHW pump electric energy), Row 31 (CHW pump frictional losses), Row 32 (CDW pump heat loss to surroundings), Row 33 (CHW heat loss to surroundings), Row 34 (CDW pump frictional losses to cooling towers), Row 35 (CHW pump frictional losses to chilled water loop), Row 37 (energy extracted by chilled water loop to chiller), Row 38 (chiller electric energy consumption), Row 39 (Cooling tower fans electric energy consumption), Row 40 (Cooling tower fans electric energy), Row 41 (energy exctracted by chillers to cooling towers), Row 42 (Heat supplied to zones from AHU), Row 43 (Return air to plenum), Row 44 (Exhaust air), Row 45 (Energy added by heating coil to be extracted by cooling coils), Row 46 (Heat added by fans to be extracted by cooling coils), Row 47 (heat added by humidifier to be extracted by cooling coils)

\{ if(\$column_array_hvac[10] > \$column_array_hvac[24])

$\$$ column_array_hvac[44] $=0$;

\$column_array_hvac[42] = \$column_array_hvac[13] + \$column_array_hvac[14] + \$column_array_hvac[15] + \$column_array_hvac[19] + \$column_array_hvac[20] -

\$column_array_hvac[21] - \$column_array_hvac[24] - \$column_array_hvvac[25]; if(\$column_array_hvac[42] $<$ \$column_array_hvac[16]) \{ \$column_array_hvac[23] = \$column_array_hvac[16] - \$column_array_hvac[42]; \} else if (\$column_array_hvac[42] > \$column_array_hvac[16]) \{ \} \$column_array_hvac[22] = \$column_array_hvac[42] - \$column_array_hvac[16]; else

\{

\$column_array_hvac[22] =0; \} \$column_array_hvac[23] = 0; \} else if $\left(\left(\$ c o l u m n \_a r r a y \_h v a c[10]<\right.\right.$ column_array_hvac[24]) ) \{

\$column_array_hvac[46] = \$column_array_hvac[14];

\$column_array_hvac[13] =0;

\$column_array_hvac[14] $=0$;

\$column_array_hvac[15] =0;

\$column_array_hvac[16] $=0$;

\$column_array_hvac[17] =0; $\$$ column_array_hvac[18] =0; \$column_array_hvac[19] $=0$; $\$$ column_array_hvac[20] $=0$; $\$$ column_array_hvac[22] $=0$; $\$$ column_array_hvac[23] $=0$; \$column_array_hvac[42] $=0$; \$column_array_hvac[43] =0; 


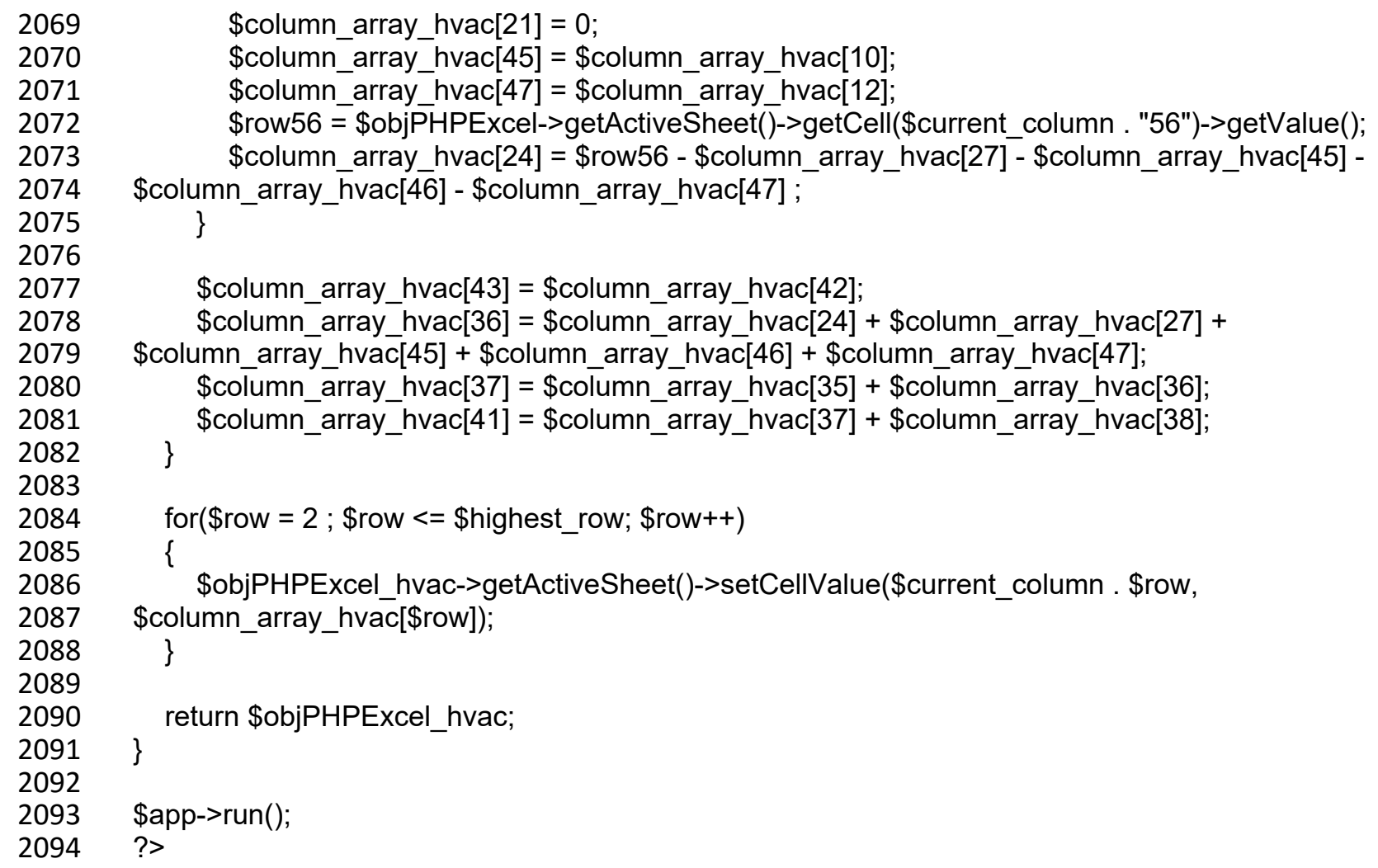




\section{Appendix G: Copyright License Agreements}

\section{Elsevier B.V.}

What follows are the rights retained by an author of an article in the journal of Energy and Buildings. A complete list of rights and further copyright details can be found on the Publisher's website at:

\section{http://www.elsevier.com/wps/find/authorsview.authors/copyright}

"As a journal author, you retain rights for a large number of author uses, including use by your employing institute or company. These rights are retained and permitted without the need to obtain specific permission from Elsevier. These include:

- the right to make copies (print or electric) of the journal article for their own personal use, including for their own classroom teaching use;

- the right to make copies and distribute copies (including via e-mail) of the journal article to research colleagues, for personal use by such colleagues (but not for Commercial Purposes**, as listed below);

- the right to post a pre-print version of the journal article on Internet web sites including electronic pre-print servers, and to retain indefinitely such version on such servers or sites (see also our information on electronic preprints for a more detailed discussion on these points);

- the right to post a revised personal version of the text of the final journal article (to reflect changes made in the peer review process) on the author's personal or institutional web site or server, incorporating the complete citation and with a link to the Digital Object Identifier (DOI) of the article;

- the right to present the journal article at a meeting or conference and to distribute copies of such paper or article to the delegates attending the meeting;

- for the author's employer, if the journal article is a 'work for hire', made within the scope of the author's employment, the right to use all or part of the information in (any version of) the journal article for other intra-company use (e.g. training), including by posting the article on secure, internal corporate intranets;

- patent and trademark rights and rights to any process or procedure described in the journal article;

- the right to include the journal article, in full or in part, in a thesis or dissertation;

- the right to use the journal article or any part thereof in a printed compilation of works of the author, such as collected writings or lecture notes (subsequent to publication of the article in the journal); and

- the right to prepare other derivative works, to extend the journal article into book-length form, or to otherwise re-use portions or excerpts in other works, with full acknowledgement of its original publication in the journal." 


\section{Appendix H: Copyright License Agreements}

\section{ACM Digital Library}

What follows are the rights retained by an author of an article in the ACM digital library. A complete list of rights and further copyright details can be found on the Publisher's website at:

\section{http://authors.acm.org/main.html}

"As an ACM author, you retain rights for a large number of author uses, including use by your employing institute or company. These rights are retained and permitted without the need to obtain specific permission from Elsevier. These include:

- Authors can post the accepted, peer-reviewed version prepared by the author-known as the "pre-print"-to the following sites, with a DOI pointer to the Definitive Version of Record in the ACM Digital Library.

- Authors can post an Author-Izer link enabling free downloads of the Definitive Version of the work permanently maintained in the ACM Digital Library

- Authors can reuse any portion of their own work in a new work of their own (and no fee is expected) as long as a citation and DOI pointer to the Version of Record in the ACM Digital Library are included.

- Authors can include partial or complete papers of their own (and no fee is expected) in a dissertation as long as citations and DOI pointers to the Versions of Record in the ACM Digital Library are included. Authors can use any portion of their own work in presentations and in the classroom (and no fee is expected). 